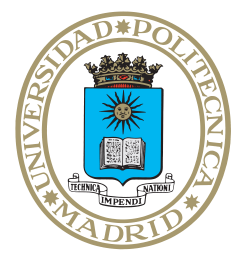

Universidad Politécnica de Madrid

Departamento de Inteligencia Artificial

DOCTORADO EN INTELIGENCIA ARTIFICIAL

\title{
A framework for ontology-based library data generation, access and exploitation
}

Doctoral Dissertation of:

Daniel Vila-Suero

Advisors:

Prof. Asunción Gómez-Pérez

Dr. Jorge Gracia 

To Adelina, Gustavo, Pablo and Amélie Madrid, July 2016 


\begin{abstract}
Historically, libraries have been responsible for storing, preserving, cataloguing and making available to the public large collections of information resources. In order to classify and organize these collections, the library community has developed several standards for the production, storage and communication of data describing different aspects of library knowledge assets. However, as we will argue in this thesis, most of the current practices and standards available are limited in their ability to integrate library data within the largest information network ever created: the World Wide Web (WWW).

This thesis aims at providing theoretical foundations and technical solutions to tackle some of the challenges in bridging the gap between these two areas: library science and technologies, and the Web of Data. The investigation of these aspects has been tackled with a combination of theoretical, technological and empirical approaches. Moreover, the research presented in this thesis has been largely applied and deployed to sustain a large online data service of the National Library of Spain: datos.bne.es. Specifically, this thesis proposes and evaluates several constructs, languages, models and methods with the objective of transforming and publishing library catalogue data using semantic technologies and ontologies. In this thesis, we introduce marimba-framework, an ontologybased library data framework, that encompasses these constructs, languages, models and methods. The specific contributions of this work include:
\end{abstract}

- marimba-datamodel, a nested relational model to represent library data sources, which can be operated using a recursive relational algebra.

- A query language, marimba-sql, which can be used to query and operate with library data in the marimba-datamodel.

- The syntax and operational semantics of a mapping language, marimba$\mathrm{rml}$, for mapping and transforming library data sources into RDF.

- Methodological guidelines for library ontology development and mapping library data sources into RDF, as well as the design, implementation and publication of a large library ontology, the BNE ontology.

- A method for extracting latent topics from ontologies and understanding thematical relationships between ontologies, as well as several topicbased ontology similarity measures.

- An in-depth study of the impact of semantic technologies in online library applications with regards to efficiency, usability and user satisfaction measures by comparing two large-scale library systems. 



\section{Contents}

List of Figures $\quad \mathbf{x}$

List of Tables $\quad$ xiv

1 Introduction 1

1.1 Challenges . . . . . . . . . . . . . . . . . 2

1.2 Context of the thesis $\ldots \ldots \ldots \ldots \ldots$

1.3 Relevant Publications . . . . . . . . . . . . . . . . . . 7

1.3.1 International Journals $\ldots \ldots \ldots \ldots$

1.3.2 International Conferences . . . . . . . . . . . 7

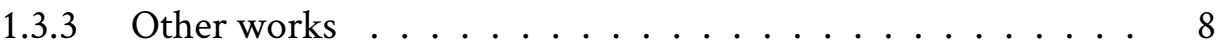

1.4 Structure of the document $\ldots \ldots \ldots \ldots$

2 State of the art $\quad 11$

2.1 Technological context . . . . . . . . . . . . . 11

2.1.1 Library science and technologies . . . . . . . . . . . . 11

2.1.2 The World Wide Web . . . . . . . . . . . . . . . . . 19

2.1 .3 Semantic Web ................... 22

2.1.4 Library ontologies . . . . . . . . . . . . . . . . . . . 29

2.2 Methods for transforming library catalogues . . . . . . . . . . . . 34

2.2 .1 Discussion . . . . . . . . . . . . . . . . . 39

2.3 Ontology-based data access . . . . . . . . . . . . . . . 40

2.3.1 Discussion . . . . . . . . . . . . . . . 48

2.4 Methods for ontology development . . . . . . . . . . . . . . . 49

2.4.1 Methodologies for ontology development . . . . . . . . . . 49

2.4.2 Lightweigth approaches for building ontologies . . . . . . . 51

2.4.3 The NeOn methodology: An overview . . . . . . . . . . . 52

2.4.4 Methods for ontology reuse . . . . . . . . . . 55 
2.4 .5 Discussion $\ldots \ldots \ldots \ldots \ldots \ldots \ldots$

2.5 Definitions ......................... 59

3 Hypotheses and objectives $\quad 61$

3.1 Open research questions $\ldots \ldots \ldots 61$

3.2 Hypotheses . . . . . . . . . . . . . . . . 63

3.3 Assumptions and restrictions . . . . . . . . . . . . . 63

3.4 Research methodology . . . . . . . . . . . . . . . . . . 64

3.5 Main contributions . . . . . . . . . . . . . . . 65

3.5.1 Constructs and models . . . . . . . . . . . . . . . 65

3.5.2 Methods and instantiations . . . . . . . . . . . . 65

3.6 Research objectives and outcomes . . . . . . . . . . . . . . 67

3.7 Marimba framework overview . . . . . . . . . . . . . . 68

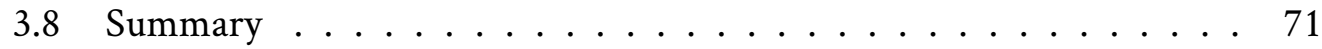

4 Mapping $\quad 73$

4.1 Contributions . . . . . . . . . . . . . . 73

4.2 marimba-datamodel . . . . . . . . . . . . 75

4.2.1 Definitions for the Nested Relational Model . . . . . . . . . . 75

4.2.2 Representing MARC 21 records in the marimba-datamodel . 77

4.3 Schema extraction . . . . . . . . . . . . . . . . . . . 82

4.3.1 Information in MARC 21 records . . . . . . . . . . 82

4.3.2 Method ................... . 84

4.4 marimba-sql query language . . . . . . . . . . . . . 86

4.4.1 Recursive algebra for the marimba-datamodel . . . . . . . 86

$4.4 .2 \quad \mathrm{SQL} / \mathrm{NF} \ldots \ldots \ldots \ldots \ldots$

4.4.3 marimba-sql syntax . . . . . . . . . . . . . 92

4.4.4 marimba-sql to recursive algebra translation . . . . . . . 93

4.5 marimba-rml language . . . . . . . . . . . . . . . 93

4.5.1 marimba-rml syntax . . . . . . . . . . . . . 94

4.5.2 marimba-rml processor . . . . . . . . . . 98

4.5.3 Evaluation of Term Maps: the Generated RDF Term of a Term Map . . . . . . . . . . . . . . . . 100

4.5.4 Building the joint query $\ldots \ldots \ldots \ldots 10 \ldots$

4.6 Mapping template generation . . . . . . . . . . . . 105

4.6.1 Mapping templates . . . . . . . . . . . . 105 
4.6.2 Templates structure . . . . . . . . . . . . . 106

4.7 Summary . . . . . . . . . . . . . . . . . . . 109

5 Ontology development 111

5.1 Contributions . . . . . . . . . . . . . . . . 112

5.1.1 Elicitation and evolution of requirements . . . . . . . . . 112

5.1.2 Active participation of domain experts . . . . . . . . . . 114

5.1 .3 Ontology publication activity . . . . . . . . . . . 117

5.2 Ontology life-cycle model . . . . . . . . . . . . . . . . . . . 117

5.2.1 Ontology life-cycle model for datos.bne.es . . . . . . . . . . . 121

5.3 First phase: Initialization . . . . . . . . . . . . . . . . . 122

5.4 Second phase: Ontology reuse . . . . . . . . . . . . . . 125

5.5 Third phase: Ontology merging and localization . . . . . . . . . 126

5.5 .1 Ontology merging . . . . . . . . . . . 126

5.5.2 The BNE ontology . . . . . . . . . . . . . . . . . . . . 129

5.6 Fourth phase: publication and evaluation . . . . . . . . . 130

5.6.1 Ontology publication . . . . . . . . . . . . . . . 130

5.6 .2 Ontology evaluation . . . . . . . . . . . . 132

5.7 Summary . . . . . . . . . . . . . . . . . 137

6 Topic-based ontology similarity 139

6.1 Introduction to topic models . . . . . . . . . . . . . . . . . . . 140

6.2 Contributions . . . . . . . . . . . . . . . . . . . . . . 142

6.3 Notation and definitions . . . . . . . . . . . . . . . . 144

6.3.1 Ontology documents . . . . . . . . . . . . . . . . . . 144

6.3.2 Topic models . . . . . . . . . . . . . . . . . . 145

6.3.3 Topic-based ontology similarity . . . . . . . . . . . 147

6.4 Marimba Topic Model . . . . . . . . . . . . . . . . . . . . . . . 148

6.4.1 Ontology document extraction . . . . . . . . . . . . . . . 149

6.4.2 Word-senses annotation . . . . . . . . . . . . . . . 149

6.4 .3 Topic modelling . . . . . . . . . . . . . . . . 151

6.4.4 An application of Marimba Topic Model . . . . . . . . . 154

6.5 Evaluation . . . . . . . . . . . . . . . . . 156

6.5.1 Training corpus . . . . . . . . . . . . 157

6.5.2 Experiment 1. Topic coherence evaluation . . . . . . . 158

6.5.3 Experiment 2. Task-based evaluation . . . . . . . . . . . 159 
6.6 Discussion . . . . . . . . . . . . . . . . . . . . 164

6.6.1 Topic coherence . . . . . . . . . . . . . . 165

6.6.2 Task-based evaluation: LOVTAGS and LODTHEMES . . . . 166

6.7 Summary . . . . . . . . . . . . . . . . . . 168

7 Generation and publication: datos.bne.es 169

7.1 Overview of the datos.bne.es project . . . . . . . . . . . . 170

7.2 Methodology . . . . . . . . . . . . . . . 172

7.3 Specification . . . . . . . . . . . . . . 173

7.3.1 Identification and analysis of data sources . . . . . . . . 173

7.3.2 IRI and URI design . . . . . . . . . . . . . . . . . . 173

7.4 RDF Generation . . . . . . . . . . . . . . 176

7.4.1 Configuration of RDF generation technologies: marimba-tool 176

7.4.2 Mapping sources into RDF . . . . . . . . . . . . . . 180

7.5 Data linking and enrichment . . . . . . . . . . . . . . . . 180

7.5.1 Identifying target datasets for linking . . . . . . . . . 181

7.5.2 Discovering and validating the outgoing links . . . . . . . 181

7.5.3 Data enrichment . . . . . . . . . . . . . . 182

7.6 Data curation . . . . . . . . . . . . . . . . . 182

7.6.1 Identifying data issues . . . . . . . . . . . . . 183

7.6.2 Reporting and fixing data issues . . . . . . . . . . . . . . 184

7.7 Publication . . . . . . . . . . . . . . . . . 184

7.7.1 Publishing the dataset on the Web . . . . . . . . . . . . 184

7.7.2 Publishing metadata describing the dataset . . . . . . . 185

7.8 Exploitation: datos.bne.es service . . . . . . . . . . . . . 187

7.8.1 Data format: JSON-LD . . . . . . . . . . . . . 187

7.8.2 Architecture . . . . . . . . . . . . . 188

7.8 .3 User interface . . . . . . . . . . . . . . . . . . 194

7.9 Summary . . . . . . . . . . . . . . . . . . 194

8 User-centered evaluation 195

8.1 Evaluation objectives . . . . . . . . . . . . . . . . 196

8.2 Experiment 1. Task-based experiment . . . . . . . . . . . . . . 197

8.2.1 Experimental settings _ . . . . . . . . . . . . . . 197

8.2.2 Evaluation measures . . . . . . . . . . . . . 198

8.2.3 Online application and data collection . . . . . . . . . . . 199 
8.2 .4 Subjects . . . . . . . . . . . . . . . . . . 199

8.2 .5 Scenario and tasks . . . . . . . . . . . . . . 200

8.2.6 Visualization of the experimental results . . . . . . . 202

8.2 .7 Experimental results . . . . . . . . . . . . . . . . . 204

8.3 Experiment 2. Usability and user satisfaction evaluation . . . . . . . . 212

8.3.1 Methodology and experimental setting . . . . . . . . 212

8.3.2 Results . . . . . . . . . . . . . . . . . 213

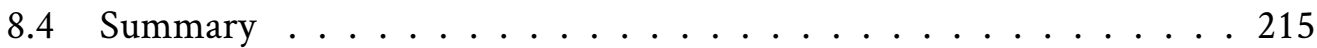

9 Conclusion $\quad 217$

9.1 Hypotheses and contributions . . . . . . . . . . . . . 217

9.1.1 Systematically analyzing and modelling library catalogue data using ontologies . . . . . . . . . . . . . . 217

9.1.2 Formally defining the mapping and transformation rules . . . 221

9.1.3 Understanding the impact of ontology-based library data access in end-user applications . . . . . . . . . . . . . . 223

9.2 Impact . . . . . . . . . . . . . . . . . . . . . . . . . 224

9.3 Future directions . . . . . . . . . . . . . . . . 226

9.3.1 Query and mapping languages . . . . . . . . . . . . 226

9.3.2 Machine learning and probabilistic approaches . . . . . . . 227

9.3.3 Data linking and enrichment . . . . . . . . . . . . . . 228

9.3.4 End-user library applications and their evaluation . . . . . 229

A marimba-sql BNF 232

Bibliography 233 



\section{List of Figures}

2.1 Marc 21 Bibliographic record corresponding to an edition from 1605 of Don Quixote de la Mancha. . . . . . . . . . . . . . . . . . 15

2.2 Marc 21 Authority record corresponding to Miguel de Cervantes Saavedra. 16

2.3 FRBR entities of Group 1 and Group 2, and their "primary" and "responsibility" relationships (Based on the Functional Requirements for Bibliographic Records [2009]). . . . . . . . . . . . . . . . . . 17

2.4 Components of a HTTP URI: Miguel de Cervantes in Wikipedia . . . . . 21

2.5 Abstract RDF Graph composed of two triples. The first triple states that Miguel de Cervantes is the creator of the work Don Quixote de la Mancha. The second triple indicates the language in which Don Quixote was written. The dashed line highlights each triple. . . . . . . . . . . 23

2.6 R2RML main components (based on Das et al. [2012]). . . . . . . . . . . 42

2.7 R2RML Term maps (based on Das et al. [2012]). . . . . . . . . . . . . 43

3.1 The marimba framework and its components . . . . . . . . . . 66

3.2 Overview of our framework . . . . . . . . . . . . 70

4.1 marimba-mapping overview. . . . . . . . . . . . . . . . 74

4.2 Table representation of two MARC 21 bibliographic records in the Nested Relational Model (we denote this table as the relation bib). . . . . . . . 78

4.3 Example of the leader field and its representation as relation-valued at-

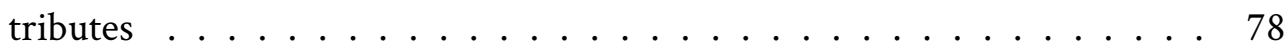

4.4 Example of two single-value control fields corresponding to the attomic attributes f001 and fo05 . . . . . . . . . . . . . . . . . 79

4.5 Example of a fixed-length control field corresponding to the relation-value attribute f008. The symbol \# is used for undefined values in the MARC 21 standard. Positions 35-37 indicate the language of the resource, Spanish in the example. . . . . . . . . . . . . . . . . . . 79 
4.6 Example of a non-repeatable data field and its representation a relationvalued attribute . . . . . . . . . . . . . . . . . 80

4.7 Example of two tuples of a repeatable data field and its representation as relation-valued attribute . . . . . . . . . . . . . . . 80

4.8 Relation resulting from applying a projection operation to bib of Fig-

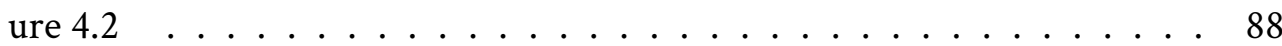

4.9 Classification mapping template . . . . . . . . . . . . . . 107

4.10 Annotation mapping template . . . . . . . . . . . . 108

4.11 Relation extraction mapping template . . . . . . . . . . . . . . 109

5.1 marimba-modelling Ontology Requirements specification activity. Adapted from Suárez-Figueroa et al. [2009]. . . . . . . . . . . . . . . . . . . 114

5.2 Sample of the mapping templates used during the ontology development for the datos.bne.es project. The coloured/grey columns indicate the different manual inputs of the domain experts during the ontology development process. . . . . . . . . . . . . . . . . . 115

5.3 marimba-modelling ontology life-cycle . . . . . . . . . . . 120

5.4 Ontology life-cycle model followed during the datos.bne.es project. The first stage included the first and second phases of the life-cycle. The second stage included the first, second, third and fourth phases of the life-cycle. 121

5.5 Core elements of the FRBR-based ontology (Vila-Suero et al. [2013]). . . 127

5.6 Core of the BNE ontology. Each class and object property includes a descriptive label and the IRI in the Compact IRI syntax, where the bne prefix corresponds to http://datos. bne.es/def/ . . . . . . . . . 131

5.7 Screenshot of the online HTML documentation page of the BNE ontology available at http://datos.bne.es/def/ . . . . . . . . . . . . . . 133

5.8 Summary provided by the online tool OOPS! before the first iteration for the BNE ontology. (Screenshot obtained from http: //oops . linkeddata. es) . . . . . . . . . . . . . . . . . . . 135 
6.1 Graphical model representation of the conditional dependencies of LDA. Each node is a random variable. The latent variables are unshaded and correspond to the topic proportions $\theta_{d}$, the topic assignments for each word $Z_{d, n}$, and the topic distributions $\phi_{k}$. The observed variables are shaded and correspond to the words of each document $W_{d, n}$. The variable $\alpha$ determines the topic distribution for each document. The rectangles are in plate notation and denote replication (Adapted from Blei [2012]) . . . 146

6.2 Graphical model representation of the conditional dependencies of BTM. Each node is a random variable. The latent variables are unshaded and correspond to the global topic proportions $\theta$, the topic assignments for each biterm $Z_{b}$, and the topic distributions $\phi_{k}$. The observed variables are shaded and correspond to each pair of words in the corpus corpus biterm set $B, W_{i}$ and $W_{j}$. The variable $\alpha$ parameterizes the global topic distribution. The rectangles are in plate notation and denote replication (Adapted from Cheng et al. [2014]) . . . . . . . . . . . . . . . . 147

6.3 An example of using topic distributions to cluster ontologies. Two-dimensional representation of distances among ontologies obtained by applying multidimensional scaling (MDS) (Kruskal [1964]) to the divergence matrix based on the Jensen-Shannon divergence for every pair of ontologies. The distances between the ontologies in the $\mathrm{X}$ and $\mathrm{Y}$ axes provide an idea of the approximated distances in a two-dimensional space. . . . . . . . . . 155

6.4 Coherence scores $\mathrm{C}(\mathrm{T} ; \mathrm{K})$ results for $L D A, L D A_{S}$ and marimba-topicmodel with $K$ from 10 to 60 . A larger value for the coherence score indicate more coherent topics. The average of ten runs with each method is shown. . . 160

6.5 An example of using topic distributions to cluster the ontologies tagged as Geography in the LOV corpus. Two-dimensional representation of distances among ontologies obtained by applying multidimensional scaling (MDS) (Kruskal [1964]) to the divergence matrix based on the JensenShannon divergence for every pair of ontologies. The distances between the ontologies in the $\mathrm{X}$ and $\mathrm{Y}$ axes provide an idea of the approximated distances in a two-dimensional space. . . . . . . . . . . . . . 167

7.1 marimba-tool modules. . . . . . . . . . . . . . . . . . 177

7.2 Architecture of the datos.bne.es service. . . . . . . . . . . . . . . . . 189

7.3 Screenshot of the datos.bne.es search interface showing the results of the user query "Aristoteles" (http://datos.bne.es/find?s=aristoteles) 192 
8.1 Relationship between tasks and core terms of the ontology introduced in Chapter 5. . . . . . . . . . . . . . . . . . . . . . . . . . 201

8.2 Average time to complete all the tasks, showing the average time required for each task. . . . . . . . . . . . . . . . . . 205

8.3 Task completion time and visited pages required to complete the tasks proposed to the participants. Each dot represents a user. As the 95\% confidence intervals overlap less than $50 \%$, the differences between the systems are significative and we can reject that differences are due to statistical fluctuations of the samples. . . . . . . . . . . . . . 206

8.4 Effect of the age. There is a statistical significant increment for datosbne participants in the year range $30-65 \ldots$. . . . . . . . . . 207

8.5 Effect of the experience on OPAC. There is no significant relation related to experience on OPAC. . . . . . . . . . . . . . . . . 208

8.6 Effect of the experience on datos.bne. There is no significant relation related to experience on datos.bne. . . . . . . . . . . . . . . . . 208

8.7 Enhancement in task completion time (TCT) required to achieve a task and pages visited (TPV) for the systems compared (OPAC and datos.bne). The error bars correspond to standard deviation (most external), 95\% confidence interval, and standard error (most inner bars) . . . . . . . . . . . 210

8.8 Effect of the course. We do not find a significant segmentation by course. 211

8.9 Average satisfaction after completing all the tasks, showing the average satisfaction in the range used by the SUS questionnaire. The differences in the average values for both systems are statistically significant. . . . . . 211

8.10 Usability and satisfaction tests' results. The horizontal lines show average and standard deviation of each system (computing all questions). Dashed lines are for datos.bne and dotted lines are for OPAC user group. on average, datos.bne show better results than OPAC user group, for both tests, but differences are not significant because most black error bars (standard error bars) of the compared systems overlap. . . . . . . . . . . . . 214

8.11 Average satisfaction per task for OPAC and datosbne users . Notice that the average values of this coarse-grain satisfaction measure are aligned with the values obtained in the overall facet of the fine-grain satisfaction shown in Figure 8.3.1. Results are also consistent with Figure 8.9. . . . . 215

9.1 Statistics of daily users of the datos.bne.es and catalogo.bne.es portals coming directly from search engines. . . . . . . . . . . . . . . . . 225 


\section{List of Tables}

3.1 Aligment of the research and technological objectives, hypotheses, artifact components and their evaluation. . . . . . . . . . . . . . 69

4.1 Complete mapping of the MARC 21 standard elements to the marimbadatamodel .......................... 81

5.1 Filling card of the Ontology publication activity . . . . . . . . 118

5.2 BNE Ontology OSRD summary . . . . . . . . . . . . . 123

6.1 Excerpt of Bibo and $\mathrm{FaBiO}$ ontology documents annotated with the wordsenses annotation method. The prefix bn: corresponds to http://babelnet.org/rdf/

6.2 Results of the ontology clustering experiment for our task-based evaluation using distance measures. The value of the $\mathrm{H}$ score $\mathrm{H}(\mathrm{G})$ for each method and gold-standard dataset are shown. Lower values of the $\mathrm{H}$ score indicate that the method is more precise. For marimba-topicmodel the average of ten runs is shown, with the standard deviation in brackets. . . 165

6.3 Results of the ontology clustering experiment for our task-based evaluation using distance metrics. The value of the $H$ score $H(G)$ for each method and gold-standard dataset are shown. Lower values of the $\mathrm{H}$ score indicate that the method is more precise. For marimba-topicmodel the average of ten runs is shown, with the standard deviation in brackets. . . 165

7.1 Description of the data sources for datos.bne.es 2.0 dataset. . . . . . . . . 174

8.1 Description of tasks comprising the scenario . . . . . . . . . . 203 




\section{Introduction}

For centuries, libraries have collected, organized, preserved and made available a substantial part of the ever-growing human knowledge. To keep up with this growth, the library and information science discipline have developed a rich theoretical framework. In one of these seminal works, Ranganathan [1931] masterfully synthesized the aim of any library into five simple laws: $i$ ) books are for use, ii) every reader his/her book, iii) every book its reader, iv) save the time of the reader, and $v$ ) the library is a growing organism. To serve these laws, modern libraries preserve, classify and catalogue hundreds of millions of information resources of any kind, from books to sound recordings to cartographic materials. With the explosion of the digital era, libraries have moved toward digital solutions to cope with such volumes of information. Nowadays, libraries process and store data into digital catalogues. These catalogues support essential services for their users, such as Online Public Access Catalogues (OPAC) that help users in locating the online library materials. While these services partially fulfill some of the current goals of libraries, it has been argued (W3C [2011]) that library catalogues remain largely disconnected from the largest information network ever created: the World Wide Web (WWW).

The World Wide Web, or simply the Web, connects people and information together at an unprecedented scale. The Web revolution started with large search engines indexing and providing access to billions of documents, and continued with the so-called "social Web", when users themselves became information publishers. More recently, the Web is evolving into a global space where not only people and documents are connected (Heath and Bizer [2011a]), but also data and applications. In this context of "openness" (Huijboom and Van den Broek [2011]) and "linked data", libraries world-wide have identified a great opportunity to modernize and evolve their services. 
Indeed, initiatives like Europeana ${ }^{1}$ are revolutionizing the way users and applications reach and interact with cultural data. Europeana gives access to millions of artworks, books, videos and sounds from museums, libraries, and archives across Europe. Libraries themselves are opening up their catalogues on the Web and linking them to other library catalogues, with notable examples like the Library of Congress of the United States of America ${ }^{2}$, the Bibliothèque nationale de France ${ }^{3}$, and the Deutsche Nationale Bibliothek ${ }^{4}$. As a consequence, there is a renewed interest from libraries in ontologies to model library catalogs, with existing standards such as the Functional Requirements for Bibliographic Records (FRBR) transformed into ontologies, or a large initiative from the Library of Congress to develop a new bibliographic framework (BIBFRAME ${ }^{5}$ ). The main objective of these initiatives is to use ontologies and the W3C Resource Description Framework ${ }^{6}$ (RDF) to transform library data into a more semantic and reusable representation. However, as any other conceptual and technological shift, bringing library catalogues to the Web poses several challenges to institutions and researchers. We ellaborate on these challenges in the next section.

\subsection{Challenges}

The aim of this thesis is to propose theoretical foundations and technological solutions to address a core open research problem: the principled transformation of library catalogues to rich ontology-based data, and their publication and exploitation on the Web. In particular, the scope of our work is centered around four related research challenges that we formulate as follows:

i) How to extract the semantics of library data? A neccesary step in the process of transforming library catalogues is to identify the data elements the library records contain. The logical structure of information in library catalogues is complex and is defined by a very large number of rules and combinations of metadata elements. Therefore, identifying the data elements that are contained within library catalog records is challenging.

\footnotetext{
${ }^{1}$ http: //www. europeana.eu/ (Last viewed 13th May 2016)

${ }^{2}$ http://id.loc.gov/ (Last viewed 13th May 2016)

${ }^{3}$ http: //data.bnf. fr (Last viewed 13th May 2016)

${ }^{4}$ http: //www.dnb.de/ (Last viewed 13th May 2016)

${ }^{5}$ https: //www. loc.gov/bibframe/ (Last viewed 13th May 2016)

${ }^{6}$ https: //www.w3.org/RDF/ (Last viewed 13th May 2016)
} 
On the one hand, library standards define a large number of different metadata fields and subfields, and many of them are rarely used in instantiated data. In her article "Marc21 as Data: A start", Coyle [2011] highlights the need of a principled analysis of the MARC 21 format, the core format for library catalogues. She presents her initial effort and suggests that not only the definitions in the standard should be analyzed, but "real" or instantiated data as well (i.e., the data within the records). Our thesis represents the first research work to explore the hypotheses posed by Coyle [2011].

On the other hand, data within catalogue records is also complex and its structure evolves through time, and with changes in the cataloguing practices and information systems. A library is a living organization, which impacts the practices followed by its cataloguers and the metadata they produce. This dinamicity must be taken into account in order to design a method for transforming catalogue data into a richer semantic representation.

In summary, the understanding of the semantics of library catalogue data and standars remains a challenge. In this thesis, we investigate novel methods and techniques to analyze the semantics of library catalogues that take into account: $i$ ) the logical structure of library records, and $i i)$ the structure of the instantiated data within the records.

\section{ii) How to structure the library data transformation and ontology development} processes? As discussed above, library catalogues and cataloguing standards are complex and highly specialized. Even if a method for transforming library catalogues is able to extract and provide information about structural and usage patterns within a catalogue, such information will not be easy to understand by professionals outside the library domain. The process of using these patterns for modelling catalogue information using ontologies can be understood as a knowledge acquisition problem (Wang et al. [2006]), where modelling experts need to collaborate with domain experts (i.e., library experts) in order to map existing catalog information into a new data model. Although existing ontology engineering methodologies such as the NeOn methodology (Suárez-Figueroa et al. [2015]) include the knowledge acquisition and elicitation activities in the ontology design process, their direct application to our problem raises two major challenges.

First, the collaboration between domain experts and ontology engineers in current methodologies for knowledge acquisition is rather static, taking the form of interviews, elicitation documents, and meetings where engineers gather information about the data model. Given the complexity and dinamycity of the information struc- 
ture in library catalogues, this "monolithic" approach is problematic due to the cost of management, dedicated time and invested effort it would require.

Second, the dinamicity of the content of records and the cataloguing practices to produce these records hinders the comprehension of the data model by ontology engineers without a continous collaboration with domain experts. Moreover, even when using the same standards, the information structure of catalogues varies from one library organization to another. In this context, managing typical documentation such as Ontology Requirements Specification Documents (ORSD) is challenging and requires the domain experts to easily communicate their knowledge about the catalogue structure.

In summary, there is a lack of mechanisms to enable an effective and efficient collaboration between ontology engineers and domain experts. In this thesis, we propose novel methodological and technological contributions to enable the transformation of library catalogues taking into account: $i$ ) "real" data from the catalogue to understand the dinamicity of information, and $i i$ ) the collaboration between domain experts and ontology engineers in the process.

Moreover, when modelling data with ontologies, one can either create new terms or re-use terms from existing ontologies. Current methodologies for ontology engineering and linked data publication include the re-use of existing terms as a core activity in the modelling process. In the library domain, as highlighted by the final report of the W3C Library Linked Data Incubator group (W3C [2011]), the library community should promote the coordinated re-use or extension of existing element sets over the creation of new sets from scratch. In this context, initiatives like $\mathrm{LOV}^{7}$ (Linked Open Vocabularies) are useful to help data publishers to find ontologies and terms, continuing the investigation started in the Semantic Web community with notable examples of ontology repositories and search engines such as Falcons by Cheng et al. [2008] and Watson by d'Aquin and Motta [2011]. However, these tools are limited to deal with ontologies in domains where there is a proliferation of ontologies that might overlap and cover different aspects of the domain.

In particular, over the years a number of ontologies for representing library data have been created. Currently, more than thirty ontologies are available, making it difficult to choose between the terms they offer. For example, the Functional Requirements for Bibliographic Records (FRBR) model (IFLA [2009]), has been expressed in a number of different ontologies, and these different expressions are not always explic-

\footnotetext{
${ }^{7}$ http: //lov. okfn.org (Last viewed 13th May 2016)
} 
itly aligned (Dunsire and Willer [2010]). While traditional search approaches, such as those offered by existing tools, are useful for finding terms, they provide limited support to understand the similarities and the topics described in library ontologies.

In summary, there is a lack of sophisticated mechanisms to support users in understanding and comparing the different ontologies that have been created to model library information. We investigate in this thesis novel methods that go beyond classical term-based comparisons and search methods for ontologies in the library domain.

iii) How to provide principled and interoperable framework for library data mapping and transformation? Over the last years, several mechanisms have been designed to transform different types of data into ontologies, with notable examples such as the W3C R2RML ${ }^{8}$ (Relational to RDF Mapping Language), a language for expressing customized mappings from relational databases to RDF datasets and W3C GRRDL $^{9}$ (Gleaning Resource Descriptions from Dialects of Languages), a technique for obtaining RDF data from XML (eXtensible Markup Language) documents. Moreover, these specifications have been accompaigned by an increasing ecosystem of tools enabling the transformation of information sources in the aforementioned formats.

In the library domain, although several tools and frameworks have been developed to transform library catalogues into RDF, each tool defines its own transformation or mapping specification mechanism. Frameworks such as Metafacture ${ }^{10}, M A R C 2 R D F$ toolkit $^{11}$, EasyM2R ${ }^{12}$, and Catmand $u^{13}$ define their own mechanism for specifiying the transformations. Moreover these mechanisms are not easily reusable by third party applications as they lack well-defined, machine interpretable meaning. This situation limits the development of an ecosystem of tools and methods that can interoperate.

In summary, there is a lack of well grounded and standard-based constructs and languages for representing library catalogue mappings and transformations. In this thesis, we investigate the extensions needed in existing standard constructs and languages to support the mapping and transformation of library data into RDF.

\footnotetext{
${ }^{8}$ https: //www.w3.org/TR/r2rml/ (Last viewed 13th May 2016)

${ }^{9}$ https: //www.w3.org/TR/grddl/ (Last viewed 13th May 2016)

${ }^{10}$ https://github.com/culturegraph/metafacture-core/wiki (Last viewed 13th May 2016)

${ }^{11}$ https://github.com/digibib/marc2rdf (Last viewed 13th May 2016)

${ }^{12}$ https://github.com/cKlee/easyM2R (Last viewed 13th May 2016)

${ }^{13}$ https: //gi thub.com/LibreCat/Catmandu (Last viewed 13th May 2016)
} 
iv) What is the impact of ontologies and semantic technologies in online library applications? The application of ontologies, RDF and linked data to library catalogues comes with the promise of providing enhanced access, user experiences and reuse of library assets on the Web, as reflected in the final report of the W3C LLD Incubator Group (W3C [2011]) and Alemu et al. [2012]. However, little research has been conducted to study the impact of these novel approaches at a wide scale and especially regarding "real-world" uses and tasks. As stated by Takhirov [2013] "no in-depth studies have been performed on how to actually deploy FRBR-inspired applications to end-user applications such as library catalogs available online".

In this thesis, we perform an in-depth user-oriented study and evaluation of a large application supported by the ontology-based models, constructs, and methods investigated in this thesis.

\subsection{Context of the thesis}

The investigation of this thesis has been tackled with a combination of theoretical, technological innovation, and empirical approaches. Moreover, the research presented in this document has been applied, deployed, and is currently used in a production system to sustain the linked data project of the National Library of Spain: the datos. bne.es project. ${ }^{14}$ The datos.bne.es data service has been online since 2014, as of April 2016 it receives more then 45,000 online visitors per month, and provides access to more than nine million resources of the library catalogue. The service is powered by the marimba-framework, which integrates the core theoretical and technological contributions of this thesis.

Moreover, the work presented in this thesis have been partially developed during three research visits:

- INRIA - Grenoble, France (2012): Research on mapping heterogeneous data to ontologies under the supervision of Dr. Jérôme Euzenat.

- Open University - Milton Keynes, United Kingdom (2012): Research and development of the transformation methods from library data into RDF under the supervision of Dr. Mathieu d'Aquin.

- INRIA - Grenoble, France (2013): Research on multilingual aspects of Linked Data generation and linking under the supervision of Dr. Jérôme Euzenat.

\footnotetext{
${ }^{14}$ http: //datos.bne.es (Last viewed 13th May 2016)
} 


\subsection{Relevant Publications}

Significant parts of the work presented in this thesis have been published in refereed international journals and conferences, as well as other in peer-reviewed fora. The corresponding publications and the related parts of the thesis are listed below.

\subsubsection{International Journals}

- Daniel Vila-Suero and Asunción Gómez-Pérez. datos.bne.es and marimba: an insight into library linked data. Library Hi Tech, 31(4):575-601, 2013.[VilaSuero and Gómez-Pérez [2013]].

- Daniel Vila-Suero, Boris Villazón-Terrazas, and Asunción Gómez-Pérez. datos.bne.es: A library linked dataset. Semantic Web Journal, 4(3):307-313, 2013. [Vila-Suero et al. [2013]].

\subsubsection{International Conferences}

- Ricardo Santos, Ana Manchado, and Daniel Vila-Suero. Datos.bne.es: a LOD service and a FRBR-modelled access into the library collections. In IFLA World Library International Conference. Cape Town, South Africa, 2015. [Santos et al. [2015]].

- Daniel Vila-Suero, Víctor Rodríguez-Doncel, Asunción Gómez-Pérez, Philipp Cimiano, John McCrae, and Guadalupe Aguado-de Cea. 3ld: Towards high quality, industry-ready linguistic linked linguistic data. European Data Forum 2014, 2014.[Vila-Suero et al. [2014b]].

- Asunción Gómez-Pérez, Daniel Vila-Suero, Elena Montiel-Ponsoda, Jorge Gracia, and Guadalupe Aguado-de-Cea. Guidelines for multilingual linked data. In 3rd International Conference on Web Intelligence, Mining and Semantics, WIMS '13, Madrid, Spain, June 12-14, 2013, page 3. [Gómez-Pérez et al. [2013]].

- Elena Montiel-Ponsoda, Daniel Vila-Suero, Boris Villazón-Terrazas, Gordon Dunsire, Elena Escolano Rodríguez, and Asunción Gómez-Pérez. Style guidelines for naming and labeling ontologies in the multilingual web. In Proceedings of the 2011 International Conference on Dublin Core and Metadata Applications. Dublin Core Metadata Initiative, 2011. [Montiel-Ponsoda et al. [2011]] 
- Boris Villazon-Terrazas, Daniel Vila-Suero, Daniel Garijo, Luis M. VilchesBlazquez, Maria Poveda-Villalon, Jose Mora, Oscar Corcho, and Asunción GomezPerez. Publishing linked data-there is no one-size-fits-all formula. European Data Forum, 2012. [Villazón-Terrazas et al. [2012]]

- Jorge Gracia, Elena Montiel-Ponsoda, Daniel Vila-Suero, and Guadalupe Aguado-de-Cea. Enabling language resources to expose translations as linked data on the web. In Proceedings of the Ninth International Conference on Language Resources and Evaluation (LREC-2014), Reykjavik, Iceland, May 26-31, 2014., pages 409-413. [Gracia et al. [2014a]]

- Jorge Gracia, Daniel Vila-Suero, John Philip McCrae, Tiziano Flati, Ciro Baron, and Milan Dojchinovski. Language resources and linked data: A practical perspective. In Knowledge Engineering and Knowledge Management - EKAW 2014 Satellite Events, VISUAL, EKM1, and ARCOE-Logic, Linköping, Sweden, November 24-28, 2014. pages 3-17. [Gracia et al. [2014b]]

\subsubsection{Other works}

- Daniel Vila-Suero. W3C Library Linked Data Incubator Group: Use cases Report. 2011.

- Daniel Vila-Suero, Asunción Gómez-Pérez, Elena Montiel-Ponsoda,Jorge Gracia, and Guadalupe Aguado-de-Cea. Publishing linked data on the web: The multilingual dimension. In Paul Buitelaar and Philipp Cimiano, editors, Towards the Multilingual Semantic Web, pages 101-117. Book chapter. Springer Berlin Heidelberg. 2014 [Vila-Suero et al. [2014a]]

Finally, a large part of the technological solutions proposed in this thesis have been licensed to the National Library of Spain:

- Daniel Vila-Suero, Asunción Gómez-Pérez, and Oscar Corcho. MAriMbA 1.0.0. Software registered in the Spanish intelectual property rights registry of Spain under the reference number M-7312-2014. Licensed in 2015 for the exploitation by the National Library of Spain. 


\subsection{Structure of the document}

The rest of the document is organized as follows:

Chapter 2: State of the art. We review the main areas related to the work of this thesis. We start by introducing the main aspects of the following domains: $i$ ) library and information standards, and ii) ontologies, semantic Web and linked data. In this chapter, we also provide a detailed review of the state of the art in our three core lines of research: $i$ ) methods for transforming library catalogues; ii) ontology-based data access; and iii) ontology development.

Chapter 3: Research objectives and contributions. We describe our research objectives and formulate our main hypotheses. Moreover, we discuss our research methodology that follows the design-science method proposed by von Alan et al. [2004], which consists in designing and building a set of constructs, models, methods and instantiations to evaluate our hypotheses. Finally, we briefly introduce our ontology-based library data framework for transforming and exploiting library data sources on the Web.

Chapter 4: Mapping. This chapter presents our main contributions for representing the mappings of library sources into RDF. First, the chapter details the method for automatically extracting mapping templates from library-information sources, their validation, their representation, and the participation of domain experts. Moreover, the chapter provides a formalization of our proposed mapping and query languages, by extending existing standards and formal frameworks. We provide an analytical evaluation of the mapping language.

Chapter 5: Ontology development. This chapter introduces the core contributions related to the methodological aspects of library ontology development. This chapter describes our proposed ontological life-cycle model for developing an ontology network that models library-related information. We evaluate our contribution by applying the life-cycle model to build and evaluate a large ontology network for the National Library of Spain,

Chapter 6: Topic-based ontology similarity. We present a method for modelling topics within corpora of ontologies. This method can be used to facilitate the comparison and selection of ontologies through topic-based similarity measures. 
We evaluate our method and the similarity measures by comparing them to other state-of-the-art approaches with regard to different evaluation measures.

Chapter 7: Generation and publication: datos.bne.es. This chapter details the instantiation of the theoretical and technological framework proposed in this thesis. This instantiation provides support to a large-scale dataset from the National Library of Spain. We discuss every method and model in the context of a realworld solution.

Chapter 8: User-centered evaluation. This chapter discusses two user-centered experiment with the goal of studying the impact of the application of our artifact at a wide scale. We develop a task-based experiment and measure several aspects in a "real-world" setting with 70 participants. Moreover, we carry out an in-depth usability study with 36 participants. Specifically, we compare the application datos.bne.es with the existing solution for searching and browsing the National Library of Spain catalog.

Chapter 9: Conclusion. We summarize the contributions and limitations of this thesis and outline directions for future work. 


\section{State of the art}

This thesis aims at providing theoretical foundations and technical solutions to tackle some of the challenges in bridging the gap between two research areas: library science and technologies, and the Web of Data. In Section 2.1 of this chapter, we review relevant notions related to these two areas. In the remaining sections, we provide a detailed review of the state of the art in our three core lines of research: $i$ ) methods for transforming library catalogues (Section 2.2); ii) ontology-based data access (Section 2.3); and iii) ontology development (Section 2.4)

\subsection{Technological context}

In this section, we introduce several concepts, technologies and standards related to the library and information science field. Furthermore, we introduce the core features and standards of the Web and, finally, the mechanisms that enable the representation and exploitation of semantic content on the Web.

\subsubsection{Library science and technologies}

Libraries have existed since ancient times and they have been backed by an active scientific discipline. However, their study as a scientific discipline did not start until the late XIX century when the first school of library science was founded by Melvil Dewey at Columbia University in 1887 . The field of library and information science deals with methods, techniques and tools for the collection, organization, preservation and dissemination of information resources. Libraries and their field of study have evolved since early days and their evolution has certainly accelerated with the current explosion of 
information, notably driven by web technologies, and by the evolution of user needs and profiles.

Historically, libraries have been responsible for storing, preserving, cataloguing and making information resources available to the public (e.g., books, videos, photographs, etc.). In particular, a great effort has been made to classify and organize these information resources so that they can be easily accessed by users. One of the major products of these efforts are library catalogues. In early days, library catalogues were built as a collection of physical catalogue cards. Later, with the advent of digital technologies, the library community has developed several standards for the production, storage and communication of data describing different aspects of library knowledge assets. As our work is related with digital catalogues, we review the most widely used standard for digital catalogues: the MARC 21 (MAchine Readable Cataloging) standard. Libraries across the world use MARC 21 to create library records and national libraries collect millions of records in this standard.

Additionally, there are two main standards that provide rules for defining the content of MARC 21 records: the Anglo-American Cataloguing Rules (AACR) and ISBD (International Standard for Bibliographic Description). In the context of this thesis, we focus on the international standard, but due to its alignment with AACR, our work can also be applied to records that follow the AACR rules. In the remainder of this section, we introduce the MARC 21 standard and provide a brief introduction to the ISBD and FRBR standards.

\section{MARC 21}

MARC 21 is a set of formats for representing and communicating machine-readable bibliographic data. The formats specify a record structure of fields and subfields, encoded using ISO 2709 (ISO 2709) and other data formats such as the XML standard. The specification defines different communication formats and currently includes formats for Authority, Bibliographic, Classification, Community, and Holdings records. We provide below a description of the logical structure of MARC 21 records, and introduce the most relevant formats for our work: the Authority and Bibliographic formats.

\section{Record logical structure}

The MARC 21 specification defines the logical structure of a machine-readable library record. Specifically, MARC records consist of three main components: the Leader, 
the Directory, and the variable fields.

Leader - The leader is composed of data elements that provide information for the processing of the record as well as descriptive information. The data elements contain numbers or coded values and are identified by relative character position. For example, positions 6 and 7 indicate the type of the record (e.g., Drawing, Manuscript, etc.). The length of the Leader is fixed to 24 characters. The meaning of each of these characters are described in the documentation of the standard . ${ }^{1}$

Directory - The directory defines a series of entries that contain the tag, length, and starting location of each variable field within the record. Each entry in the Directory has a length of 12 characters. The directory ends with a field terminator character. This component does not hold any potentially semantic information.

Variable fields - Variable fields are the containers of the data in a MARC record. Each variable field is identified by a tag composed of three numeric characters. For example, the field with tag 100 contains the established form of a personal name in a record that conforms to the format for Authority data The standard identifies two types of variable fields: control fields and data fields. Each type of variable field has different structural characteristics and purpose. Control fields contain control numbers or other coded information used for processing records but do not contain indicators or subfield codes. Data fields contain information about the resource or resources described within the record and are subdivided into one or more subfields identified by a subfield code preceded by a delimiter (e.g. \$). Additionally, some data fields are further specified by two character positions called indicator positions. The meaning of the subfield codes and the two character indicators varies according to the field tag they precede.

Control fields - Control fields contain either: $i$ ) a single data element, or ii) a series of fixed-length data elements identified by their relative character position. The control fields are identified by tags in the character range $00 \mathrm{X}$, with $\mathrm{X}$ being a numeric character ranging from 1 to 9 . For example, the control field 001 is defined in the standard as "the control number assigned by the organization creating, using, or distributing the record".

Data fields - Differently from control fields, data fields contain: $i$ ) two indicator po-

\footnotetext{
2016)

${ }^{1}$ https://www.loc.gov/marc/bibliographic/bdleader.html (Last viewed 13th April
} 
sitions placed at the beginning of the field, and $i i$ ) a series of subfield codes preceding each data element within the field.

Indicator positions. These two character positions interpret or supplement the data found in the field. The interpretation of the values in the two indicator positions is independent. Indicator values are defined by a lowercase alphabetic or a numeric character.

Subfield codes. The subfield code is intended to distinguish and interpret the data elements within a field. A subfield code is composed by: $i$ ) a delimiter character (we will represent it as \$), and ii) a data element identifier defined by a lowercase alphabetic or a numeric character (i.e., the code itself, such as $\$$ t for the title of bibliographic resource).

Field tags, subfield codes, and indicators positions are known as content designators. The main purpose of the specification is to define the meaning of the possible values for these content designators.

\section{Bibliographic format}

The Bibliographic format describes specific forms of bibliographic materials, such as books, computer files, maps, music, or visual materials. The format includes access points to the bibliographic record, such as agents associated with the resource, its subject focus, and relationships to other resources, as well as descriptive information. Access points are the fields of the record that enable users and librarians to find bibliographic records. Coded fixed-length fields with controlled terminologies cover a range of more than 100 properties such as target audience, Braille music format, and frequency of continuing resource. Variable-length fields are grouped into blocks indicating the function of the data in the context of the record, for example description (titles, edition, imprint), and the topics related to the bibliographic resource. Within each block, separate fields are allocated to specific types of property, such as title statement and edition statement. These fields are further specified by subfields such as title, statement of responsibility, and name of a part or section. The relationship between a field and its subfields is complex. Subfields can be mandatory or repeatable, and although encoded with a single alphanumeric character, they do not always follow alphanumeric order. Many fields are intended to be aggregations of the data contained in their subfields and presented as a single string. The MARC 21 Bibliographic format has evolved from the original MARC format developed by the Library of Congress in the 1960s, and it now includes over 200 fields. 


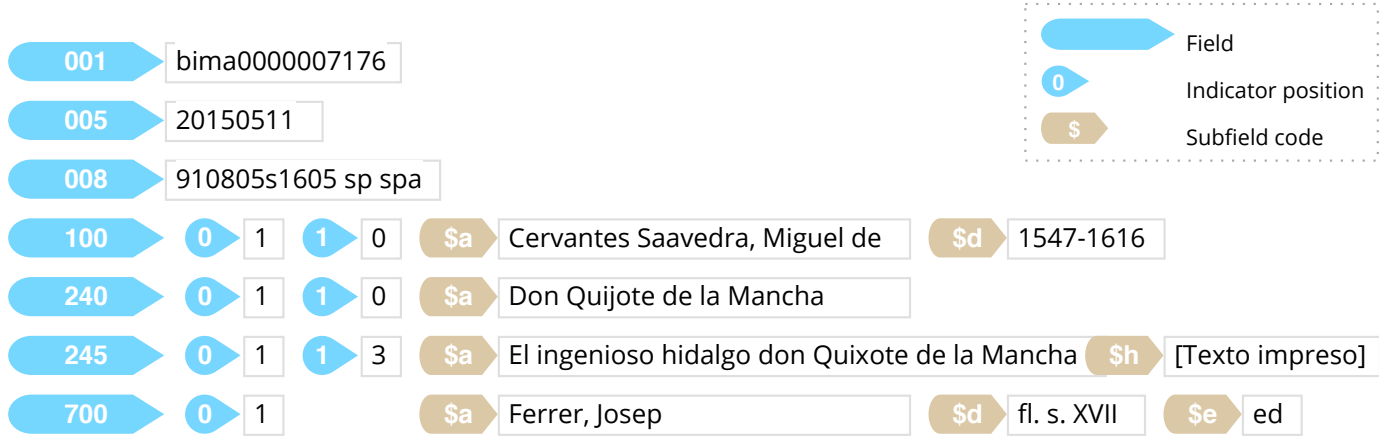

Figure 2.1: Marc 21 Bibliographic record corresponding to an edition from 1605 of Don Quixote de la Mancha.

\section{An example: Don Quixote de la Mancha}

Figure 2.1 depicts an extract from a bibliographic record produced by the National Library of Spain, corresponding to one edition of Don Quixote de la Mancha of Miguel de Cervantes. In the record, we find several control fields. For instance, the field 008 contains information regarding the language of the edition (i.e., Spanish) and other characteristics such as the type of bibliographic material (i.e., Printed text). Moreover, the record contains a number of data fields. For example, the field 100 is the access point to the record and contains information about the author of the edition, whereas the subfield \$a contains the controlled form of the author name. The name of the author is further specified in the authority record corresponding to Miguel de Cervantes shown in Figure 2.2.

\section{Authority format}

The Authority format covers records for the forms of names, subjects, and subject subdivisions used in access points of MARC 21 bibliographic records. The format specifies a structure for authorized forms with unique labels or headings, cross-references to those forms from other labels, and relationships between the forms. Name forms include the names of persons, corporate bodies, conferences, and uniform titles of bibliographic resources. Subject forms include topics, places, events, genres and forms, and the name forms used as subjects. Relationships are represented implicitly by subfields with the same field, such as personal name and title of a work, and explicitly by properties at the field level, such as the property "see also tracing for a personal 


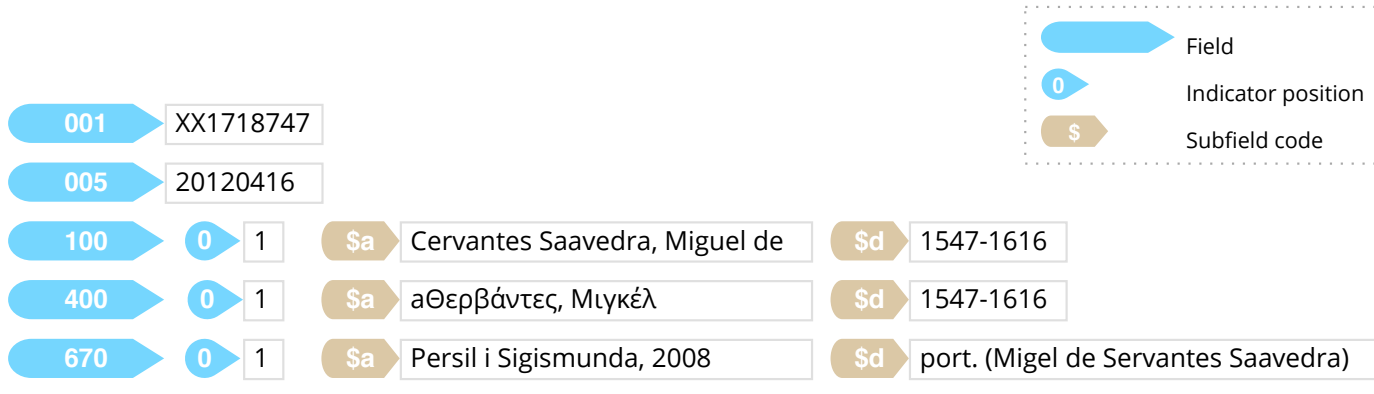

Figure 2.2: Marc 21 Authority record corresponding to Miguel de Cervantes Saavedra.

name". There are around 140 fields, such as personal name heading, occupation, and associated language.

\section{An example: Miguel de Cervantes}

Figure 2.2 depicts an extract from an authority record produced by the National Library of Spain, corresponding to the author Miguel de Cervantes. In the record we find several control fields. For instance, the field 001 contains the unique identifier in the BNE catalogue. Moreover, the record contains a number of data fields. For example, the field 100 is the main access point to the record and contains information about the main entity being described by the record (i.e., Miguel de Cervantes), whereas the subfield $\$ d$ contains information about the dates associated with the described entity (i.e., birth and death dates).

\section{$I S B D$}

The International Standard Bibliographic Description (IFLA [2011]) developed and maintained by IFLA since the 1970s specifies the description of a bibliographic resource as a specific sequence of recorded or transcribed data elements. The description is intended to be internationally acceptable and covers basic bibliographic data for all published resources across the world. ISBD does not cover access points or relationships between resources. The consolidated edition of the standard brings together separate specifications for general materials, ancient monographic publications, cartographic materials, electronic resources, monographic publications, non-book materials, printed music, and serials. The documentation lists the elements, their sequence and mandatory or repeatable status, and the punctuation used to delimit them. The 


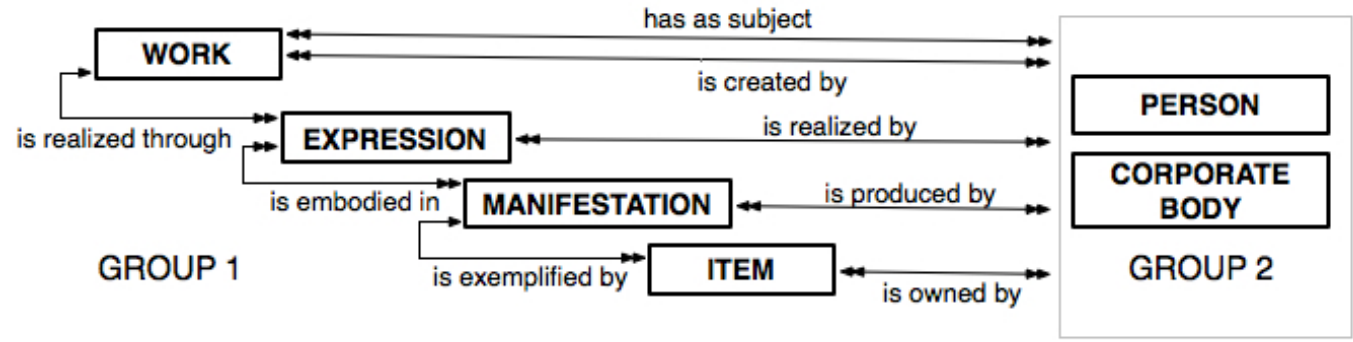

Figure 2.3: FRBR entities of Group 1 and Group 2, and their "primary" and "responsibility" relationships (Based on the Functional Requirements for Bibliographic Records [2009]).

elements cover a similar range to the MARC fields and subfields for descriptive data, such as title proper, edition statement, and equinox of cartographic resources; there are around 120 in total. The main part of the documentation consists of rules for recording the content of the elements or transcribing it from the resource being described.

\section{Functional Requirements for Bibliographic Records}

IFLA also maintains the Functional Requirements (FR) family of models for bibliographic data. There are three models, for Bibliographic Records $\left(\mathrm{FRBR}^{2}\right)$, Authority Data $\left(\right.$ FRAD $\left.^{3}\right)$, and Subject Authority Data $\left(\right.$ FRSAD $\left.^{4}\right)$. As we will discuss in this thesis, the FRBR model has generated a lot of interest in the library community. FRAD and FRSAD are more recent models that extend the FRBR model with new entities, attributes, and relationships for authority and subject data. In this section, we focus on describing the core FRBR model.

The FRBR model follows an entity-relationship design to identify the "things" described by the bibliographic data, their attributes of these "things", and their relationships to other "things". As a result, the FRBR study proposes an entity-relationship model and a set of associated user tasks (find, identify, select and obtain). In this thesis, we are mainly interested in the entities, relationships and attributes.

\footnotetext{
${ }^{2}$ http://www.ifla.org/publications/functional-requirements-for-bibliographic-records (Last viewed 13th April 2016)

${ }^{3}$ http: //www. ifla.org/publications/functional-requirements-for-authority-data (Last viewed 13th April 2016)

${ }^{4}$ http://www. ifla.org/node/5849 (Last viewed 13th April 2016)
} 
The FRBR model represents bibliographic resources using four self-contained entities, labelled work, expression, manifestation, and item with the following characteristics:

- Expressions and manifestations have primary relationship with two other components, in the order given, so that the components form a chain; for example an expression has a "realization" relationship with a work, and an "embodiment" relationship with a manifestation. These relationships also have cardinality constraints: work and expression are one-to-one, as are manifestation and item, but expression and manifestation are many-to-many.

- Each entity has a "responsibility" relationship with an agent, a person or corporate body such as a creator of a work or a publisher of a manifestation.

- Each entity is assigned a set of attributes, for example title of the work, edition or issue designation, and name of a person.

- The model also specifies bibliographic relationships between the work, expression, manifestation, and item entities, such as the summarization of one work by another, or the reproduction of a manifestation as an item.

FRBR entities represent the objects of interest to users of library data and are organized in the standard into three groups: i) Group 1, that defines the core entities for describing bibliographic resources ; ii) Group 2, that defines the core entities related to the agents involved in the creation, production, and manipulation of bibliographic resources; and, iii) Group 3, that defines the entities related to the subjects or themes of bibliographic resources. The main focus of this thesis are the first two groups: Group 1 and Group 2. Figure 2.3 provides a graphical representation of the entities and core relationships of Group 1 and Group 2. The core features of these two groups are described below.

- Group 1 entities (work, expression, manifestation, and item) represent different aspects of intellectual or artistic products. A work is an abstract entity that defines a distinct intellectual creation, which is recognized through its individual realizations or expressions. An expression is also an abstract entity and can take several forms, such as alphanumeric or musical. A manifestation is the physical embodiment of a certain expression (e.g. a certain edition of the written form of a work). Finally, an item is an entity that represents a single exemplar of a 
manifestation (e.g., one of the copies of a certain edition of the written edition of a work).

- Group 2 entities (Person and Corporate body) represent the agents involved in the creation, distribution, and dissemination of intellectual products.

Although the FRBR model has been available for more than a decade, its implementation on library systems is relatively limited (Hickey et al. [2002], Manguinhas et al. [2010], Aalberg et al. [2006]; Aalberg [2006], Takhirov et al. [2012],Simon et al. [2013]). The main problem behind its application lies in the difficulty of adapting existing catalogue data to FRBR. Typically, higher-level entities like FRBR expression or work are not made explicit within MARC 21 catalogues. MARC 21 catalogues are record-oriented and one record can describe several distinct entities (e.g., the author, the manifestation, and even the associated expression and work).

\subsubsection{The World Wide Web}

The World Wide Web (WWW) was created by Tim Berners-Lee in 1989 to enable the connection of information resources and systems within a unified information space. The Web built on the concept of hypertext, coined by Ted Nelson in 1965 . Hypertext can be simply defined as text that contains links to other text and was later extended with the notion of hypermedia to reflect that not only text can be connected through links but any other type of information resource as well, such as images or video. Whereas pioneer hypermedia systems were centralized and provided navigation accross local information resources, the Web provides a decentralized information system by abstracting the underlying network and creates a global information space. The Web can be seen as an attempt to provide a unified interface to the Internet. This interface builds on two core architectural components: the Hypertext Transfer Protocol (HTTP) and Uniform Resource Identifiers (URIs). These two components serve as the basis to identify, connect, locate and retrieve a wide variety of information resources.

\section{HTTP}

HTTP is an application-level protocol for distributed, collaborative, hypertext information systems. ${ }^{5}$ The protocol provides a common and uniform access mechanism for

\footnotetext{
${ }^{5}$ http://tools.ietf.org/html/rfc7230 (Last viewed 13th April 2016)
} 
web resources. An HTTP client is an application that communicates with an HTTP server by sending HTTP requests. An HTTP server is an application that accepts HTTP requests and sends HTTP response messages. The protocol defines the syntax and semantics of the communication but does not make any assumption on the type of resource. This loose definition allows to build a distributed and decentralized architecture where computers in different locations and with different operating systems can be used to publish, connect, navigate and retrieve disparate information resources. The protocol defines the syntax and semantics of messages and makes no assumptions about the content. But, how can applications identify (and access) these information resources on the Internet? The answer is by using Uniform Resource Identifiers.

\section{URIs, URLs and IRIs}

A Uniform Resource Identifier (URI) provides a simple and extensible means for identifying a resource. ${ }^{6}$ A resource can be anything, from an electronic document to an online service to something that is not accessible through the Internet such as a corporation, an abstract concept or a historical person. URIs provide uniform identifiers in the sense that they can be used to identify different types of resource even when the way to access them differs.

A URI is a sequence of characters chosen from a limited subset of the repertoire of US-ASCII characters. As shown in Figure 2.4, the syntax of URIs defines five components: scheme, authority, path, query and fragment. A URI scheme further restricts the syntax and semantics of the identifier. Examples of well known URI schemes are $h t t p$, that will be described later in this section, ftp for file transfer, or mailto: for sending emails. The other four components provide details that serve to identify the resource within the scope of the URI's scheme. We explain them in the context of the HTTP scheme, which is the most widely used scheme in the current Web.

URIs within the http scheme identify.potential HTTP servers listening for client connections ${ }^{7}$ The HTTP server is identified by the authority component, which consists of a host identifier such as es . wikipedia. org or an IP address (Internet Proto$\left.\mathrm{col}^{8}\right)$ such as 91.198 .174 .192 , the path and optional query component further identify a potential target resource within the namespace of the server. In summary, HTTP

\footnotetext{
${ }^{6}$ https://tools.ietf.org/html/rfc3986 (Last viewed 13th April 2016)

${ }^{7}$ HTTP servers listen for TCP (Transmission Control Protocol) https://tools.ietf.org/ $\mathrm{html} / \mathrm{rfc} 793$ connections (Last viewed 13th April 2016)

${ }^{8}$ The latest version of the Internet Protocol is version 6 https://tools.ietf.org/html/ rfc2460 (Last viewed 13th April 2016)
} 


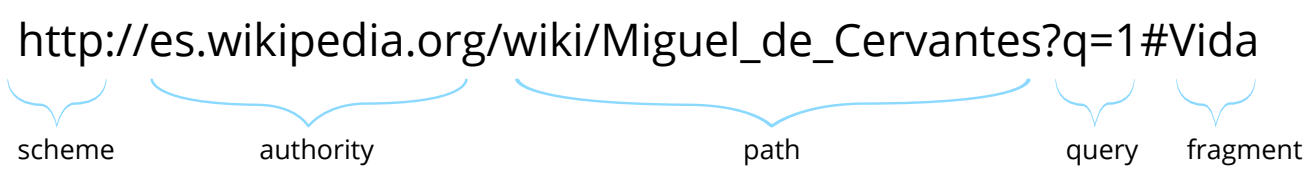

Figure 2.4: Components of a HTTP URI: Miguel de Cervantes in Wikipedia

URIs are intented to enable the retrieval of information resources on the Web by pointing clients to servers that potentially hold a resource.

It is worth noting the use of the term "potential" to reflect that the availability of a server/resource is not guaranteed. It is commonly misunderstood that URIs are only used to refer to accesible resources. URIs only provide identification, access is neither guaranteed nor implied by the presence of a URI.

Intimately related to URIs are Uniform Resource Locators (URLs). URLs are a subset of URIs that provide a means of locating a resource (e.g., its location in the network). In this sense, HTTP URIs are URLs as they provide a mechanism for locating the resources on the internet.

More recently, a generalization of URIs named Internationalized Resource Identifier 9 (IRIs) has been proposed to accomodate the increasing amount of web resources in languages with non-Latin scripts such as Japanese. The rationale is that URIs often include natural language descriptions and they are very restricted in terms of the characters they allow. IRIs generalize URIs by enabling the use of UNICODE ${ }^{10}$ characters. Following the example shown in Figure 2.4, the IRI for the work La española inglesa in the Spanish Wikipedia is: https://es.wikipedia.org/wiki/La_española_ inglesa. As the latest W3C recommendations use this more inclusive standard, we will use in this thesis IRI instead of URI whenever possible.

\section{Web resources}

The Web and the variety of resources that makes available have evolved significantly since its early days. Nevertheless, if one had to mention a predominant format that would be the Hypertex Markup Language (HTML). HTML is the main markup language for the Web. HTML documents contain links to other web resources (identified by their URLs) and enable users to go from one document to another by simply activating the links. Under the hood, when a user clicks a link, the user agent (e.g., a

\footnotetext{
${ }^{9}$ http://www. ietf.org/rfc/rfc3987 (Last viewed 13th April 2016)

${ }^{10}$ http://unicode.org/standard/standard.html (Last viewed 13th April 2016)
} 
browser), issues an HTTP request to the server identified by a URL, and receives an HTTP response containing the requested web resource. This process is also known as dereferencing a URI.

Together with HTTP and URIs, HTML is a central piece of the current Web. The widespread of HTML documents over the Web has created the largest information space ever built by humans. However, even in the original proposal of Berners-Lee, the Web was intented to connect not only human-readable documents but any other type of resource including machine-interpretable resources that can be understood by applications. Although HTML documents can be processed by applications, to derive insightful information from them remains a challenge. These documents are intented for human consumption and lack a formal mechanism to express the meaning of the information they contain. This is where the concepts of the Semantic Web and linked data come in.

\subsubsection{Semantic Web}

An approach to enhance machine understanding of web resources is to make the information they contain explicit. This approach is the vision of the Semantic Web:

"The Semantic Web is not a separate web but an extension of the current Web, in which information is given well-defined meaning, better enabling computers and people to work in cooperation” Berners-Lee et al. [2001].

From the above quote emerges a fundamental question: how can one express well-defined meaning in the context of the Web? The problem of providing well-defined and machineinterpretable meaning has been studied for decades within the area of Knowledge Representation (KR). As with hypermedia systems, KR systems prior to the Web were mostly centralized. The proposal of the Semantic Web was to bring knowledge representation efforts to a Web-scale. To this end, the Semantic Web vision adds two core elements to the existing Web architecture: the Resource Description Framework (RDF) and ontologies. 


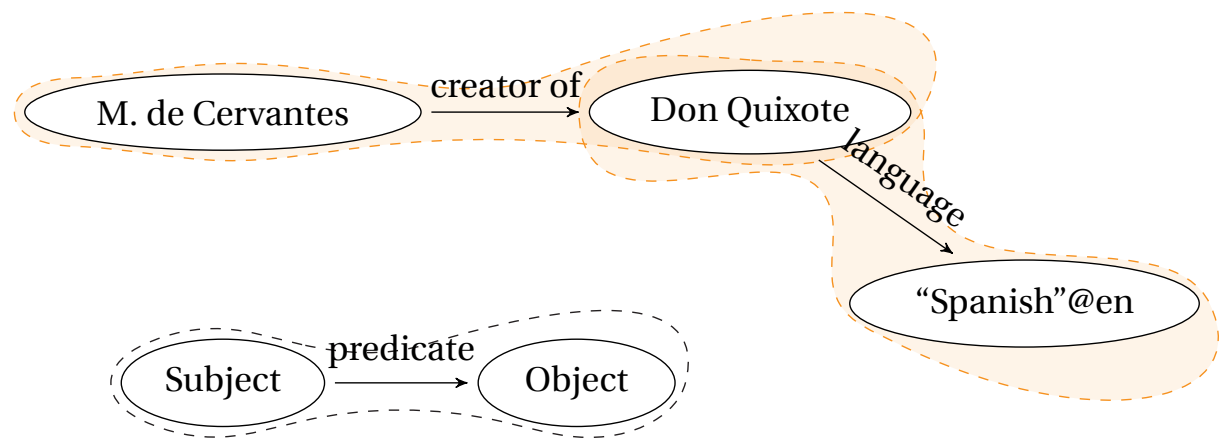

Figure 2.5: Abstract RDF Graph composed of two triples. The first triple states that Miguel de Cervantes is the creator of the work Don Quixote de la Mancha. The second triple indicates the language in which Don Quixote was written. The dashed line highlights each triple.

\section{Resource Description Framework (RDF)}

$\mathrm{RDF}^{11}$ is the fundamental knowledge representation model for the Web. The framework is composed by an abstract syntax, a formal model-theoretic semantics ${ }^{12}$, and several serializations such as Turtle and JSON-LD.

RDF represents knowledge as triples in a graph-based data model. A triple is composed of subject, predicate, and object. A set of triples is an RDF Graph. The subject and the object can be understood as nodes connected by a directed arc, the predicate. There are three kind of nodes: IRIs, Literals, and Blank nodes. To illustrate these concepts, Figure 2.5 depicts an RDF graph in abstract syntax expressing information about Miguel de Cervantes and its work Don Quixote, which is described by two triples.

IRIs are used to denote resources in the subject and predicate parts of a triple. The object can be denoted by an IRI or a Li teral. Additionally, a Li teral or literal value has a datatype. A datatype defines the range of possible values such as strings or numbers. Finally, a Literal can be tagged with a language string to indicate its natural language using ISO codes such as es for Spanish.

Blank nodes provide the ability to describe resources without assigning them a global identifier (i.e., a IRI). Blank nodes are useful when we want to describe resources that are local to a particular resource. For example, imagine that we want to describe that page 23 of a book is damaged, then it is probably not the best approach to

\footnotetext{
${ }^{11}$ http: //www.w3 .org/TR/rdf11-concepts/ (Last viewed 13th April 2016)

${ }^{12}$ http: //www.w3.org/TR/rdf11-mt/ (Last viewed 13th April 2016)
} 
create a new IRI for the page. We could instead use a Blank node to refer locally to this page and potentially to other pages of the book. In this way, the book would have its own IRI and in its RDF description it would include the Blank nodes describing further properties of the pages.

As mentioned above, several serializations for RDF have been proposed over the years. In this thesis we will use Turtle as it is relatively easy to read and it is the preferred serialization for the latest W3C recommendations. Continuing with the example of Figure 2.5, we show in Listing 2.1 the RDF graph serialized in Turtle corresponding to real triples in the DBpedia dataset ${ }^{13}$ :

Listing 2.1: RDF triples corresponding to Miguel de Cervantes in the DBpedia dataset using the Turtle RDF serialization

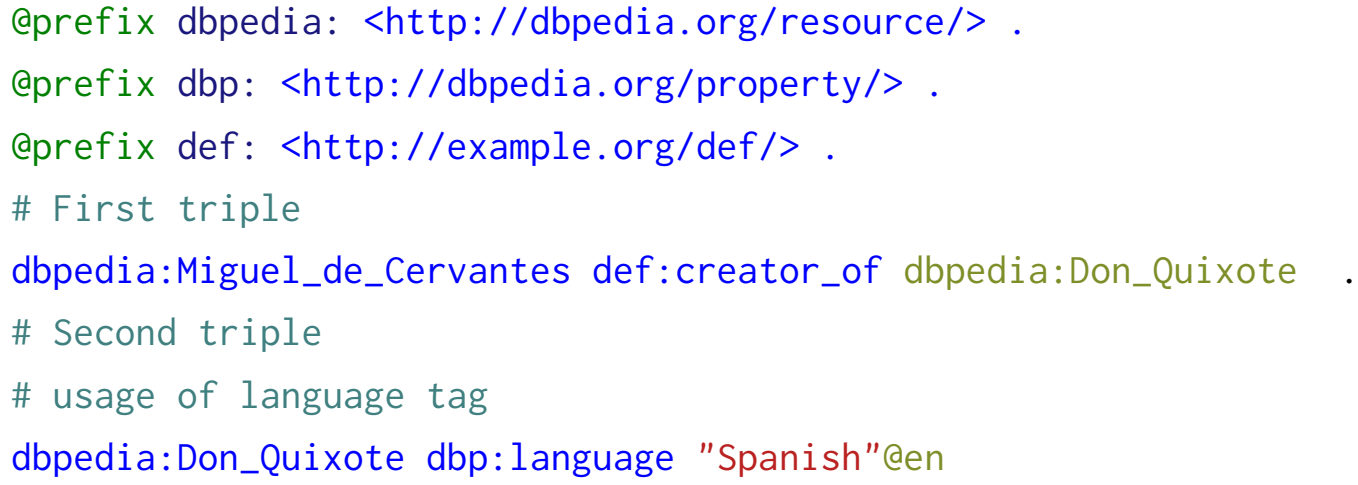

Note above how recurring parts of IRIs are declared at the top with aprefix directives, which saves space and improves clarity when there are many triples in a document. This notation is known as CURIE $^{14}$ (Compact URI) and will be the preferred notation in this thesis. The RDF graph above is composed of two triples where dbpedia:Miguel_de_Cervantes acts as the subject of the first triple, def : creator_of as the predicate indicating an authorship relation with dbpedia:Don_Quixote. Finally, the second triple states the language of the work denoted by dbpedia:Don_Quixote using a language tagged literal 'Spanish' @en.

In the example above, def : creator_of and dbp: language correspond to properties in the DBpedia data model. The DBpedia data model defines the meaning of RDF coming from DBpedia. We have explained RDF, the basic data model, and its serialization. However, there is a remaining question: how can one define data mod-

\footnotetext{
${ }^{13}$ DBpedia is an LOD dataset extracted from Wikipedia

${ }^{14}$ http: //www.w3.org/TR/curie (Last viewed 13th April 2016)
} 
els for describing information with RDF?. The answer is by using ontologies, which we describe in the next section.

\section{Ontologies}

Ontologies are explicit specifications of a conceptualization ( Gruber [1993]). A conceptualization is an abstract, simplified view of the domain that one wishes to represent for some purpose. More explicitly, an ontology is a formal specification of the entities, their properties, restrictions, and relationships within an specific domain such as cultural heritage, Middle-age architecture, or Politics. The core elements to represent knowledge in an ontology can be summarized in:

- Classes (or concepts), which represent the entities to be modelled, commonly organized in hierarchies.

- Properties (or roles, or relations), which represent types of associations between the classes in the model, or between classes and certain predefined datatypes. They can be hierarchically organized as well.

- Individuals (or instances), which represent instances of classes.

In the context of the Web, they were introduced to enable the creation of reusable and portable data models to be used together with RDF. Currently, there are two standards to create such data models, the RDF Schema (RDFS), which provides a set of minimal ontological properties, and the Ontology Web Language (OWL), which is a family of specifications some of them based on Description Logics (Baader et al. [2003]) that provide a set of more complex constructs. RDFS and OWL ontologies can be used along with information written in RDF, and the ontologies themselves are primarily exchanged as RDF documents. We briefly describe both standards below.

\section{RDFS}

The RDF Schema ${ }^{15}$ provides a data-modelling vocabulary for RDF data. RDFS provides a basic set of constructs for describing groups of related resources and the relationships between these resources. RDFS can be used to define classes, properties, hierarchies of classes and properties, and domain and range constraints for properties.

For example, an RDF Schema in Turtle for modelling authors and literary works is shown in Listing 2.2.

\footnotetext{
${ }^{15}$ http://www.w3.org/TR/rdf-schema/ (Last viewed 13th April 2016)
} 
Listing 2.2: RDF schema for modelling authors and literary works

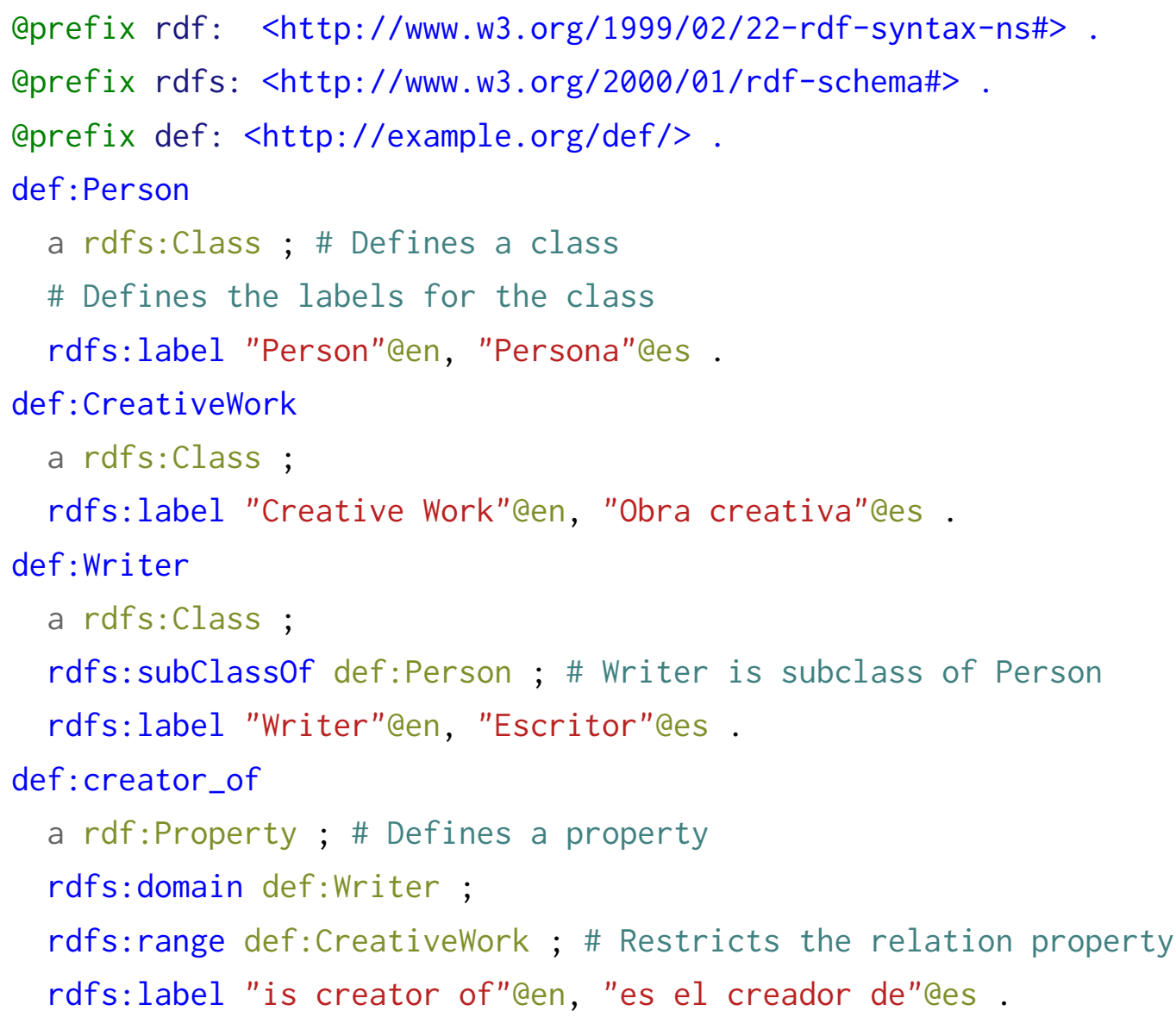

$O W L$

Although RDFS allows to build basic data models, its expressive power is limited. For example, in RDFS we cannot express that the property created by is the inverse property of creator of, or to express disjointness of classes (e.g., to express that an individual cannot be a printed book and a sound recording at the same time). To this end, OWL ${ }^{16}$ provides a series of constructs that extend RDFS to enable the construction of more expressive ontologies.

Currently, there are two versions of OWL, OWL 1 (2004) and OWL 2 (2012). The OWL 1 specification distinguished three sub-languages with different purposes, complexity and expressivity levels: Lite, DL, and Full (in ascending order of expressivity). OWL-Lite provides minimal expressivity and adds only a few constructs to RDFS. OWL DL provides maximum expressivity while still keeping decidability and

\footnotetext{
${ }^{16}$ http://www.w3.org/TR/owl2-overview/ (Last viewed 13th April 2016)
} 
completeness, and OWL Full includes all modelling constructs with no guarantee about computational decidability or completeness. Later, the OWL 2 recommendation introduced three different OWL profiles (i.e., sub-languages), EL, QL, and RL. These profiles are intented to cover different requirements and application scenarios. OWL 2 EL is well-suited for very large ontologies. OWL $2 \mathrm{QL}$ is oriented to data sets with a large number of individuals where the main reasoning task is query answering. Finally, OWL 2 RL is focused on relatively light-weight ontologies that are used to describe data sets with a large number of RDF triples and the main operations involve handling RDF triples.

Finally, RDFS ontologies can be extended with OWL constructs. We show below an example of extending our previous RDFS ontology with OWL constructs for defining an object property and an inverse relation:

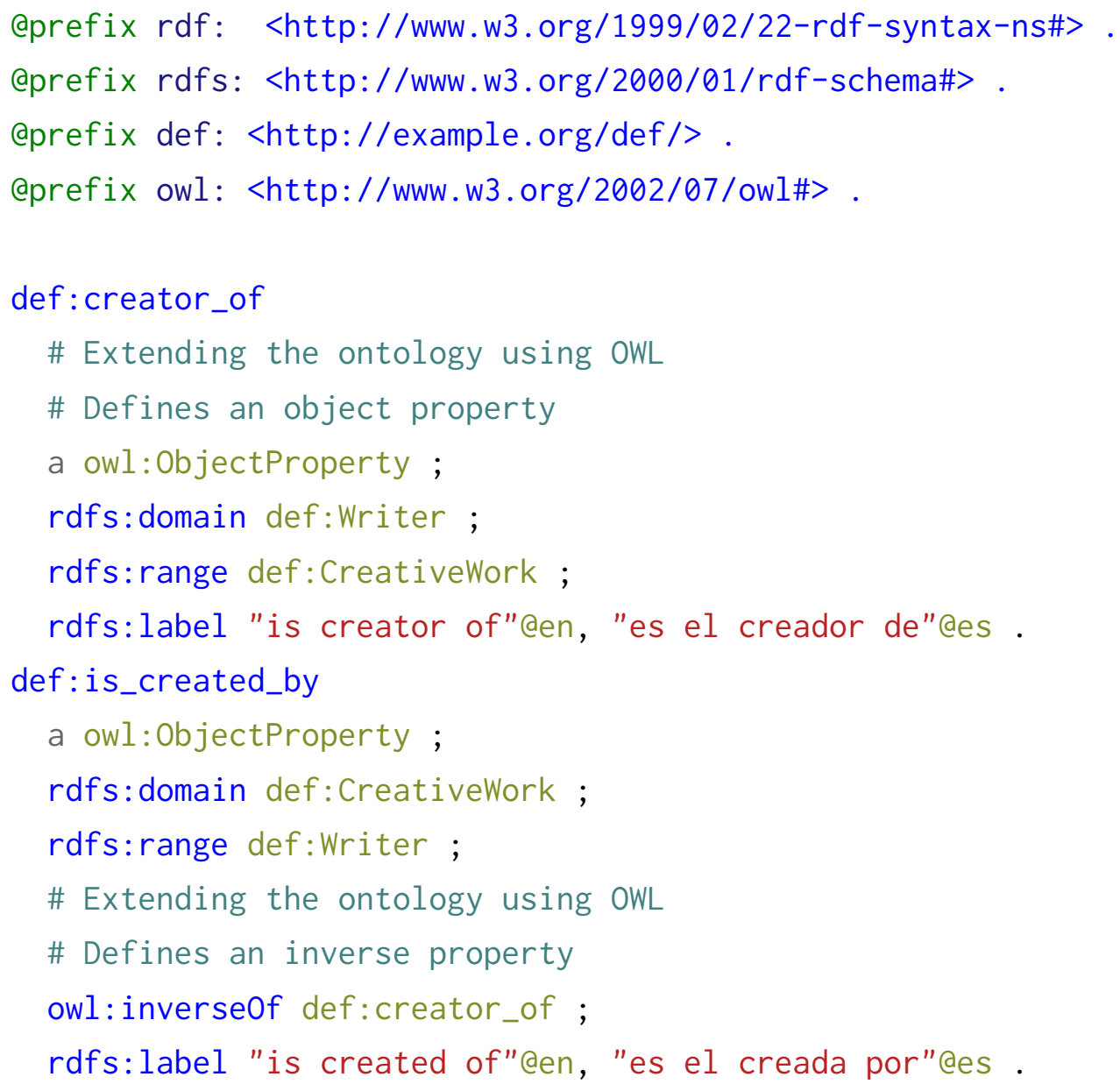

HTTP, URIs, OWL and RDF have been around for many years now, however the vision of the Semantic Web has not gained traction until recently with the increasing 
adoption of the linked data principles, that we describe in the next section.

\section{Linked data}

In the early years of the Semantic Web, the focus on intelligent agents and logics was very strong making the Semantic Web a highly specialized field with a high entry barrier. The focus was in fact more in the semantics than on the Web itself. While this situation has led to mature standards, as the ones introduced in previous sections, it also led to low adoption, and, what is more critical, to scarcity of semantic data on the Web. How can there be intelligent agents if there are not enough data and services to start with? To mitigate these issues, Berners-Lee [2006] formulated four simple "rules" that are known as the "Linked Data principles":

1. Use URIs (Uniform Resource Identifiers) as names for things.

2. Use HTTP URIs so that people can look up those names.

3. When someone looks up a URI, provide useful information, using the standards (RDF).

4. Include links to other URIs so that they can discover more things.

The first and second principles boil down to one fundamental goal: if we want clients to consume and act upon information with well-defined meaning, our resources must be identified and retrievable via the standard mechanism of the Web: HTTP. This principle might seem evident today, but many of the ontologies and individuals created during the early days of the Semantic Web did not follow this rule, leading to the aforementioned lack of availability of resources.

The third and fourth principles highlight a major question: if we want clients to benefit from data on the Web, we must provide well-defined meaning (through RDF) and enable them to retrieve more data traversing the Web of Data. Fundamental web algorithms such as PageRank by Page et al. [1997] are based on this simple principle, let clients explore the Web to make sense of information and the underlying connections.

Since 2006, these principles and what has been called the "Linked Open Data" initiative have led to a tremendous growth of RDF data on the Web. Finally, Linked Data can be currently defined as a set of principles and practices for publishing and linking structured data on the Web using web standards (Heath and Bizer [2011b]). This thesis heavily relies on a combination of these principles with ontologies. In the 
next section, we review several ontologies, which have been developed to model library data.

\subsubsection{Library ontologies}

In this section, we introduce and review core RDF vocabularies and ontologies developed within the library community and related communities like DCMI (Dublin Core Metadata Initiative) or the OAI (Open Archives Initiative).

\section{IFLA ISBD elements}

The IFLA ISBD elements vocabulary ${ }^{17}$, developed by the IFLA ISBD/XML Study Group, is a representation of the consolidated edition of the ISBD standard. This vocabulary defines a general class Resource, which represents an abstract and general conceptualization of a bibliographic resource. The vocabulary also provides 180 properties for describing bibliographic resources.

\section{IFLA FRBRer}

The IFLA FRBRer (FRBR entity-relationship) vocabulary ${ }^{18}$, developed by the IFLA Namespaces Task Group, is an ontological representation of the IFLA FRBR entity relationship model. This vocabulary formalizes the FRBR model with a high degree of fidelity (i.e., one RDFS class per entity, one RDFS property per relationship and attribute, using appropriate RDFS domains and ranges).

\section{IFLA FRAD}

The IFLA FRAD vocabulary ${ }^{19}$, developed by the IFLA Namespaces Task Group, is a representation of the IFLA FRAD entity relationship model introduced in Section 2.1.1. This vocabulary complements the FRBRer vocabulary and defines nineteen RDFS classes to model authority entities such as corporate bodies and agencies. The vocabulary also provides a wide range of additional RDFS properties to describe the attributes and relationships among FRBR entities.

\footnotetext{
${ }^{17}$ http://iflastandards.info/ns/isbd/elements/ (Last viewed 13th April 2016)

${ }^{18} \mathrm{http}$ ://iflastandards. info/ns/fr/frbr/frbrer (Last viewed 13th April 2016)

${ }^{19}$ http://iflastandards. info/ns/fr/frad/ (Last viewed 13th April 2016)
} 


\section{IFLA FRSAD}

The IFLA FRSAD vocabulary ${ }^{20}$, developed by the IFLA Namespaces Task Group, is a representation of the IFLA FRSAD entity relationship model introduced in Section 2.1.1. This vocabulary defines two RDFS classes. The class Thema, which is intended to encode any entity that is subject of a FRBR Work. The class Nomen, that is intended to encode the sequence of signs (e.g., alphanumeric) by which a Thema is known, referred to, or addressed as.

\section{FRBR Core}

The FRBR Core vocabulary ${ }^{21}$, created by Ian Davis and Richard Newman in 2009, defines core concepts and relations described in the FRBR model prior to the official release of the IFLA FRBRer vocabulary. The vocabulary includes RDFS classes for the FRBR groups 1, 2 and 3 (see Section 2.1.1), and adds some RDFS classes that are not defined by IFLA FRBR standard. The most significant class that was added is the class Endeavour, which is equivalent to the union of all items, manifestations, expressions and works. Other additions are specializations of the class Work, such as Li terary Work, and Legal Work.

\section{RDA (Resource Description and Access)}

FRBR entities for RDA. The FRBR entities for RDA vocabulary, created and managed by the DCMI/RDA Task Group ${ }^{22}$, defines the classes corresponding to FRBR entities of group 1, 2 and 3 (see Section 2.1.1) and adds the class Family included in FRAD. Also, it introduces the class Agent as the super-class of Person, Corporate Body and Family classes. Additionally, the vocabulary defines the class Subject as the superclass of Concept, Event, Object and Place classes. The set of classes may be used along with the properties defined in the RDA elements' and relationships' vocabularies that are described below.

RDA elements (group 1, group 2 and group 3). The RDA elements vocabularies, created and managed by the DCMI/RDA Task Group, define the set of properties to be used for describing instances of the classes defined in the FRBR Entities for RDA vocabulary described above.

\footnotetext{
${ }^{20} \mathrm{http}$ ://iflastandards.info/ns/fr/frsad/ (Last viewed 13th April 2016)

${ }^{21}$ http://vocab.org/frbr/core.html (Last viewed 13th April 2016)

${ }^{22}$ http://rdvocab. info/ (Last viewed 13th April 2016)
} 
RDA relationships for Work, Expression, Manifestation and Item (WEMI). The $R D A$ relationships for WEMI vocabulary, created and managed by the DCMI/RDA Task Group, is composed of the properties representing the relationships between the entities of the FRBR group 1 (see Section 2.1.1).

\section{SKOS}

The Simple Knowledge Organization System ${ }^{23}$ (SKOS) is a common data model for knowledge organization systems, such as thesauri, classification schemes, subject heading systems and taxonomies. SKOS has been widely used to model library data, especially to authority subject headings and controlled vocabularies.

\section{$M A D S / R D F$}

The Metadata Authority Description Schema in RDF (MADS/RDF) is a vocabulary ${ }^{24}$ for authority data proposed by the LoC to be used for describing subject headings and controlled value for names (of persons, organizations, places, etc.). The MADS vocabulary is specifically tailored to support authority data as understood and used by library science methods and systems. MADS/RDF includes mappings to SKOS and provides labels and descriptions only in English.

\section{OAI-ORE}

The Open Archives Initiative Object Reuse and Exchange $\left(\mathrm{OAI}-\mathrm{ORE}^{25}\right)$ is a vocabulary developed by the OAI community, to model the description and exchange of aggregations of web resources. The ORE Data Model consists of four classes. The class Aggregation, which represents a set of resources. The class Aggregated Resource, which represents a resource that belongs to an aggregation. The class Resource Map, which describes an Aggregation, the Aggregated Resources that conform it, and the relationships between them. The class Proxy, which allows to create statements about an Aggregated Resource in the context of a specific Aggregation.

\footnotetext{
${ }^{23}$ https: //www.w3.org/TR/skos-reference/ (Last viewed 13th April 2016)

${ }^{24}$ http://www. loc.gov/standards/mads/rdf/v1.html (Last viewed 13th April 2016)

${ }^{25}$ http: //www. openarchives.org/ore/1.0/vocabulary (Last viewed 13th April 2016)
} 


\section{BIBFRAME}

The BIBFRAME data model ${ }^{26}$ is one of the core outcomes of the Bibliographic Framework Transition Initiative of the Library of Congress of the United States of America (LoC). A major focus of the BIBFRAME initiative is to determine a transition path for the MARC 21 format to web-based, linked data standards. The LoC has made available an RDFS implementation of the BIBFRAME model, which is currently being discussed and improved together with the international library community. The BIBFRAME Model consists of four core classes. The class Creative Work represents resources reflecting a conceptual essence of the cataloging resource. The class Instance represents resources reflecting an individual, material embodiment of a Creative Work. The class Authority represents a resource reflecting key authority concepts that represent relationships with a Creative Work and its Instances such as people, places, topic, organizations, etc. The class Annotation represents resources that enhance the knowledge about another resource.

\section{GND Ontology}

The GND ontology $y^{27}$ is developed by the DNB to be used for describing the linked authority data of german-speaking countries, called the Gemeinsame Normdatei (GND) data set. The GND ontology is a rich ontology currently composed by 61 classes and more than 300 properties. Additionally, some of the ontology classes and properties are linked to other ontologies such FOAF (Friend of a Friend) and the RDA vocabularies through owl: equivalentClass, $r d f s$ : subclassOf, owl : equivalentProperty, and rdfs: subpropertyof statements.

\section{$\mathrm{FaBiO}$}

The FRBR-aligned Bibliographic Ontology $y^{28}$ is a vocabulary for recording and publishing descriptions of publications that contain bibliographic references. FaBiO classes are structured according to the FRBR entity-relationship model (see Section 2.1.1). The most noticeable characteristic of this vocabulary is that it adds additional properties that extend the FRBR data model through the linking of works and manifestations, works and items, and expressions and items.

\footnotetext{
${ }^{26}$ http: //bibframe.org/ (Last viewed 13th April 2016)

${ }^{27}$ http://d-nb. info/standards/elementset/gnd (Last viewed 13th April 2016)

${ }^{28}$ http: //www. sparontologies.net/ontologies/fabio (Last viewed 13th April 2016)
} 
$B I B O$

The Bibliographic Ontology $y^{29}$ is a vocabulary that can be used as a citation ontology, as a document classification ontology or, simply, as a way of describing in RDF any kind of document. The core RDFS classes defined by BIBO are following. The class Collection represents "a collection of documents or collections" and is the super class of specialized classes such as the Periodical, Series, and Website classes. The class Document which represents "a bounded physical representation of body of information designed with the capacity (and usually intent) to communicate" and is the super class of classes like the Book, Article, Map, Slideshow classes. The class Event is intended to describe bibliographic-related events such as Conferences or Hearings.

\section{Dublin Core Metadata Element Set}

The Dublin Core Metadata Element Set $^{30}$ vocabulary is a DCMI Recommendation for providing general description of resources. The element set contains fifteen properties to represent and describe a wide set of resources ranging physical to digital resources such as books, websites, or scientific articles.

\section{DCMI Metadata Terms}

The DCMI Metadata Terms ${ }^{31}$ vocabulary contains the specification of the metadata terms maintained by the DCMI. The vocabulary refines the DC Metadata Element Set with several $r d f s$ : range restrictions and a variety of new properties. The core RDF classes are Agent and Bibliographic Resource.

\section{Europeana Data Model}

The Europeana Data Model ${ }^{32}$ (EDM) ontology, actively developed in the context of the Europeana project, defines a model for collecting, connecting and enriching the descriptions provided by Europeana's cultural content providers. The EDM ontology is specifically designed to model aggregations in the Europeana platform.

As we have seen in this section there are currently a great number of ontologies for describing library linked data on the Web. Some ontologies are specifically tailored towards different types of data like for example authority data such as persons, or

\footnotetext{
${ }^{29}$ http: //bibliontology.com/ (Last viewed 13th April 2016)

${ }^{30}$ http://www. dublincore.org/documents/dces/ (Last viewed 13th April 2016)

${ }^{31}$ http: //dublincore.org/documents/dcmi-terms/ (Last viewed 13th April 2016)

${ }^{32}$ http://dublincore.org/documents/dcmi-terms/ (Last viewed 13th April 2016)
} 
organizations, or pure bibliographic data such as manuscripts, or journal. On the other hand, other vocabularies can be used for describing a wider range of data; one example is BIBFRAME that is designed to cover a broad spectrum of data. Moreover, most of them cover similar concepts with different approaches and levels of granularity, which poses a challenge for reusing the concepts of library ontologies to model library data.

\subsection{Methods for transforming library catalogues}

The main objective of this thesis is to support principled transformation of library catalogues to rich ontology-based data, and their publication and exploitation on the Web. Over the last years, several approaches have been proposed for transforming existing catalogue data into richer data models. In this section, we review the most relevant works, in chronological order, to identify the open research challenges that motivate the contributions of this thesis.

Hickey et al. [2002] reported a series of experiments with algorithms to group existing bibliographic records into FRBR works and expressions. A preliminary experiment consisted in automatically identifying expressions in a subset of bibliographic records that had been manually annotated. Their algorithm was able to achieve 0.68 precision with respect to the gold standard, identifying 28 expressions out of 41 in the gold standard. From these results, the authors concluded that although it is possible to automatically identify expressions using field-based heuristics, the information provided by these fields is often unreliable which leads to poor precision. This conclusion highlights the need of human intervention during the entity identification process.

Besides this preliminary conclusion, the main contribution of the paper is the FRBR work-set algorithm. A work-set or super work is a higher level abstraction of a work that enables the inclusion of additional formats. For instance, both the book and movie versions of Gone with the wind would be grouped together as a work-set if they both have the same title and are attributed to the same author. The authors showed that the use of an external authority file highly increased the precision of the algorithm. This conclusion highlights the positive effect of using authority files during the identification process. Moreover, the authors stressed out the importance of cataloging practices for extracting more predictable work-sets. The work-set algorithm was later updated and doc- 
umented in $2009^{33}$. In the paper, the authors thoroughly described the rules for building what they call FRBR keys. FRBR keys are built from author and/or title information and are used to group together bibliographics records.

Bennett et al. [2003] analyzed the concept of FRBR work using a sample of WorldCat, a very large collection of bibliographic records from libraries around the world. The authors argued that $i$ ) the key difficulty in working with FRBR entities lies in the concept of expression, and $i i)$ the identification of expressions presents serious implementation issues. This led the authors to focus exclusively on manifestations and works. The authors described a simple method to cluster bibliographic records into their corresponding works. Applying this method to a subset of records, they estimated that: i) 47 million bibliographic records could be linked to 32 million distinct works in Worldcat (an average work is linked to 1.5 manifestations), and ii) 78\% works were linked to a single manifestation. Although these results might indicate that the potential of FRBR was limited to approximately $20 \%$ of the works, the authors stressed out that these FRBR works are of high relevance to users based on the number of physical exemplars that they cover in the catalogue.

Hegna and Murtomaa [2003] performed an analysis of MARC data from two national bibliographies in the light of the FRBR data model. In this work, the authors analyzed the mapping from MARC data elements into FRBR entities and restricted their study to personal authors, excluding corporate authors and conferences. Specifically, the study was focused on finding and mapping the works, expressions, and manifestations contained in fields and subfields of MARC bibliographic records. The main conclusions of their study can be summarized as follows: $i$ ) the normalization of data in the form of authority records greatly helps to establish relationships between FRBR entities found in MARC records, ii) the work of catalogers and the quality of cataloging improves the ability of finding and connecting FRBR entities, and iii) the use of the entities and relationships can increase the quality of search results in end-user applications.

\footnotetext{
${ }^{33}$ Hickey, Thomas B. and Jenny Toves. 2009. FRBR work-set Algorithm. Version 2.0. Dublin, OH: OCLC Online Computer Library Center, Inc. (Research division). Published online at: http://www.oclc.org/research/activities/past/orprojects/frbralgorithm/ 2009-08.pdf. (Last viewed 13th April 2016)
} 
Aalberg et al. [2006]; Aalberg [2006] presented a tool for transforming MARC records into FRBR data. The tool is able to parse MARC records in XML and produce a set of FRBR-based records based on a set of transformation rules expressed in XSLT. ${ }^{34}$ The main objective under the development of the tool was to focus on complex relationships among the entities within bibliographic records that go beyond the simple work, expression, and manifestation hierarchy. The XSLT transformation rules are created from a conversion rules database with three tables: i) Entity mapping, for identifying entities in records, ii) relationship mapping, for extracting relationships among entities, and $i i i)$ attribute mapping, for defining properties of the identified entities. As stated by the authors, the conversion rules have to be adapted to every source catalogue to be converted, due to different cataloging practices and usages of the MARC standard. However, the involvement of cataloging experts and developers is not defined and the conversion rules are complex for non-technical users, hindering its application to large and evolving catalogues.

Finally, the process does not include any analytical step to understand the real usage of the MARC standard within the catalogue, which could help users to define the conversion rules. Similar approaches for identifying FRBR works were later proposed by Freire et al. [2007] and Manguinhas et al. [2010].

Manguinhas et al. [2010] proposed several algorithms and techniques for transforming existing MARC records from several catalogues into the FRBR model, in the context of the TELPlus project. ${ }^{35}$ Their approach makes use of MARC bibliographic and authority records. The MARC bibliographic records are transformed into the FRBR model using the FRBR core ontology. ${ }^{36}$

The main contributions of the work are: i) working with multilingual data, ii) transforming several catalogues, eventually created with different cataloging practices, and iii) focusing not only on FRBR works but also on expressions. Their approach consists of three main steps: i) pre-processing, ii) FRBR entity extraction, and iii) FRBR aggregation.

The pre-processing step applies a set of rules that adapt and enrich bibliographic records using authority data. In order to define the rules to be applied, the

\footnotetext{
${ }^{34}$ W 3C XSL Transformations recommendation for transforming XML documents (see https: // www.w3.org/TR/xslt) (Last viewed 13th April 2016)

${ }^{35}$ http: //www. theeuropeanlibrary.org/telplus/ (Last viewed 13th April 2016)

${ }^{36}$ http://vocab.org/frbr/core.html (Last viewed 13th April 2016)
} 
authors previously performed an analytical study using the source records. The FRBR entity extraction step is based on a set of rules that are programatically defined, which poses a technical challenge for cataloging experts to define new rules. The FRBR aggregation step deals with clustering the resulting FRBR entities in order to reduce the number of duplicates.

The authors applied their approach to a collection of 94,831 bibliographic and 5,970 authority records from 12 libraries. Using the transformed collection, they performed an evaluation with end-users using the resulting data. In particular, library users and professionals were asked to use two different search systems: $i$ ) a traditional OPAC system, and ii) a FRBR-based system that grouped the results based on FRBR entities. After using the system, the users completed a survey composed of 5 questions specifically dealing with the impact of FRBR on search results. From the results, the authors concluded that most users preferred the FRBR-based system. Although the results were interesting, the study showed a lack of details such as the experimental setting, or whether the users were asked to complete specific tasks, among others. Finally, the authors also highlighted the importance of exploiting authority records during the transformation process.

Takhirov et al. [2012] proposed a framework called FRBR-ML ${ }^{37}$ for transforming and enriching legacy bibliographic information into a representation where the structure and semantics of FRBR model is explicit. FRBR-ML design is driven by three features: $i$ ) usage of a simple and understandable machine-readable format, which the authors argue is XML, $i i$ ) fidelity to the FRBR model, and $\mathrm{iii}$ ) exchange between the FRBR-ML and MARC formats. The framework focuses exclusively on the MARC bibliographic format and does not exploit records in the authority format.

The main contributions of this work are $i$ ) an exchange format from MARC to FRBR in XML, and $i i$ ) semantic enrichment of records. Regarding the semantic enrichment of records, the authors define a function that applies two different techniques. On the one hand, a dictionary-based technique, that consists of a lookup to a table of mappings between field/subfield code combinations (e.g., 245\$a), and target semantic elements (e.g., Title). On the other hand, a knowledge-based technique that performs queries to several knowledge bases,

\footnotetext{
${ }^{37}$ FRBR-ML stands for FRBR in XML
} 
ranks the resulting entities, and assigns the semantic type of best ranked entity to the target entity (e.g., a Person). The main limitation of this technique, according to their experiment with a small subset of records using Freebase and DBpedia, is that almost half of the queries did not yield any result. Moreover, it is unclear $i$ ) how the extracted types are used to establish relationships and create entities in the FRBR-ML process, and $i i$ ) what are the fields used to build the queries. Finally, although the experimental results are interesting, they are limited in scope and the authors admit that "the user feedback from librarians is important and should help detect the potential weaknesses and advantages of the approach in real world settings .

Simon et al. [2013] described the approach followed to build and publish the LOD service of the National Library of France. ${ }^{38}$ This project deals with the transformation of MARC bibliographic and authority records to RDF following the FRBR model. The conversion and publication system is built using CubicWeb ${ }^{39}$, an open source software. CubicWeb uses a relational database in the backend and an ad-hoc query language, RQL (Relational Query Language) to generate different representations of resources (e.g., in HTML, JSON, or RDF).

The data model or schema is written in Python and the approach is to keep a strongly typed data model (i.e., in a relational database), and to render RDF using different ontologies. The main limitation of this approach is the lack of mechanisms to participation of library experts: $i$ ) to evolve the mappings with the different ontologies, and $i i)$ to provide feedback to refine the data schema. Evolution of mappings has to be done programatically on both the relational database model and the RDF views.

Moreover, the authors detailed the process of identifying and linking FRBR works. Their approach used machine learning to predict whether an unlinked manifestation can be linked to one of the existing works. Finally for linking to external entities, the system uses an alignment tool called Nazca. ${ }^{40}$ Unfortunately, experimental results and associated data are not reported in the paper and are not available on the Web.

marc2bibframe tool More recently, there has been an increasing interest from the community in the BIBFRAME model. A major focus in the development of

\footnotetext{
${ }^{38}$ http://data.bnf.fr (Last viewed 13th April 2016)

${ }^{39}$ https://www. cubicweb.org/ (Last viewed 13th April 2016)

${ }^{40}$ https://demo. cubicweb.org/nazca/ (Last viewed 13th April 2016)
} 
BIBFRAME is the interoperability with legacy MARC systems. To experiment with the transformation of MARC catalogues into BIBFRAME, the marc2bibframe ${ }^{41}$ tool has been made available to the community. The tool is an XQUERY utility that transforms MARC/XML records into BIBFRAME RDF/XML data. Like other approaches, the transformation rules are defined programatically and technical knowledge is required to refine them for different catalogues.

\subsubsection{Discussion}

In the previous section, we have described the most relevant related works for the transformation of library catalogues into richer data models. For each of the approaches, we have highlighted the open research challenges. In this section, we summarize these challenges and relate them to the novel contributions of this thesis.

Extensive use of FRBR as a data model. The FRBR model has been the preferred choice when transforming library catalogues into richer data models. Several studies already point out the potential benefits, but deeper studies are needed (Bennett et al. [2003], Manguinhas et al. [2010]). However, modern transformation approaches should be prepared to adapt to emerging models such as RDA and BIBFRAME.

Use of authority files improves the quality. Most works highlight the importance of using authority files during the transformation process (Hegna and Murtomaa [2003], Hickey et al. [2002]).

Application of standards varies for each catalogue. Every time a catalogue is to be transformed to a richer data model, a significant effort must be put into understanding how the cataloging standards are used and into refining the transformation rules accordingly (Manguinhas et al. [2010], Takhirov et al. [2012]).

Library experts feedback during the process is crucial. Heterogeneity of cataloging practices highly impacts the process of transformation (Hegna and Murtomaa [2003], Takhirov et al. [2012]). Most of the works agree that library experts can provide valuable feedback to ease the process, overcome this heterogeneity, and improve the quality of the results. However, none of the approaches proposes systematic mechanisms to benefit from such feedback. Moreover, the modification and evolution of rules in existing works is done programatically (Hickey

\footnotetext{
${ }^{41}$ https://github.com/lcnetdev/marc2bibframe (Last viewed 13th April 2016)
} 
et al. [2002], Manguinhas et al. [2010], Simon et al. [2013]), or using technical standards such as XSLT or XQUERY transformation rules (Aalberg et al. [2006]; Aalberg [2006], Takhirov et al. [2012], marc2bibframe). This poses a barrier for non-technical library experts, limiting the capacity of the approaches to benefit from their feedback.

Transformation rules must be defined iteratively and incrementally. Due to the heterogeneity within library catalogues, most of the works stress out the need of evolving and refining the mapping and transformation rules. Interestingly, one of the most exhaustive approaches (Manguinhas et al. [2010]) is based on a previous analytical study. However, no systematic approach to this analytical step is found in the state of the art.

Lack of interoperability of mapping and transformation rules. Every work in the literature proposes its own methods for defining transformation rules, limiting the ability to reuse them accross institutions and catalogues. Moreover, these ad-hoc mechanisms cannot benefit from existing standards and tools proposed for the Semantic Web, such as W3C R2RML, the relational databases to RDF mapping language.

Lack of evaluation results. Only Manguinhas et al. [2010] performed an evaluation of their approach for transforming library catalogues into the FRBR model. However, their study is not reproducible and does not provide sufficient details about their experimental setting and the evidences obtained from the experiment.

\subsection{Ontology-based data access}

As we have discussed in the previous section, there is lack of interoperable languages for defining mapping and transformation rules for MARC 21 data sources. In this section, we review existing work in the area of ontology-based data access. Mapping data from one data model to another is an important task of many data integration applications. Data integration applications typically deal with heterogeneous data models that need to be analyzed and matched either manually or semi-automatically. Over the years, many lines of research have focused on this problem, such as ontology matching (Shvaiko and Euzenat [2013]), schema integration in the field of databases (Batini et al. [1986b]), and ontology-based data access (Poggi et al. [2008]). Ontology Based 
Data Access (OBDA) is an approach to data integration based on providing a unified view over data sources using ontologies (Poggi et al. [2008]). The OBDA problem can be loosely defined by the tuple $\mathcal{G}, \mathcal{S}, \mathcal{M}$, where $\mathcal{G}$ is a global schema, $\mathcal{S}$ is the source schema, and $\mathcal{M}$ is the mapping between $\mathcal{S}$ and $\mathcal{G}$. Moreover, the problem involves one or several data source and target layers. Nowadays, the predominant data model in OBDA for the target layer is RDF. Regarding the source layer, a major focus of OBDA research has been put on relational databases, thus the source schema $\mathcal{S}$ is frequently a relational database schema. Nevertheless, more recently there has been an increasing focus on other data sources such as XML, JSON, and CSV.

The mapping layer defines the relation between the target and the source layers. There are various ways for defining mappings. Three well known approaches are: $i$ ) Local as View (LAV), where each element of $\mathcal{S}$ is mapped to a query over $\mathcal{G}$ (Ullman [2000]); ii) Global as View (GAV), where each element of $\mathcal{G}$ is mapped to a query over $\mathcal{S}$ (Halevy [2001]); and iii) Global Local As View (GLAV), which combines the GAV and LAV approaches by defining each query over $\mathcal{S}$ as a query over $\mathcal{G}$ (Friedman et al. [1999]). Another important aspect related to mappings is the formalism or language to define the mapping rules. In this section, we discuss various approaches that differ in the source formats and schemas, and specially in the mapping language and approach. In this review, we focus on the latest W3C standards and their recent extensions, which are based on prior approaches, notably $\mathrm{R}_{2} \mathrm{O}$ by Barrasa et al. [2004] and D2RQ by Bizer and Seaborne [2004]. For a complete survey on mapping relational databases into RDF, we point the reader to the survey by Sahoo et al. [2009].

W3C Direct Mapping - The Direct Mapping recommendation ( Sequeda et al. [2012], Arenas et al. [2013]) defines an automatic procedure for transforming relational databases into RDF without user-defined mapping rules. The input of this procedure is a relational schema $\mathcal{R}$, and an instance $\mathcal{I}$ of $\mathcal{R}$. The output is an RDF graph described with an OWL ontology.

The direct mapping recommendation defines a set of transformation rules: $i$ ) Row node rule, to define the subject of the triples in the RDF graph ; ii) Table triples generation rule, to assign an $\mathrm{rdf}$ : type based on the table name; iii) Literal triples generation rule, to generate data properties based on the table and column names ; and, iv) Reference triples generation rule, to establish relationships based on the foreign keys found in the tables.

W3C R2RML - The R2RML (RDB to RDF) mapping language provides a set of 
constructs to transform relational databases into RDF (Das et al. [2012]). The mapping rules are defined in an R2RML mapping document using Turtle.

An R2RML mapping document contains a set of Triples Maps ( $r r$ :TriplesMap) that are used to generate RDF triples from the tables of the source database. Figure 2.6 presents the main elements of an R2RML mapping document that we introduce below.

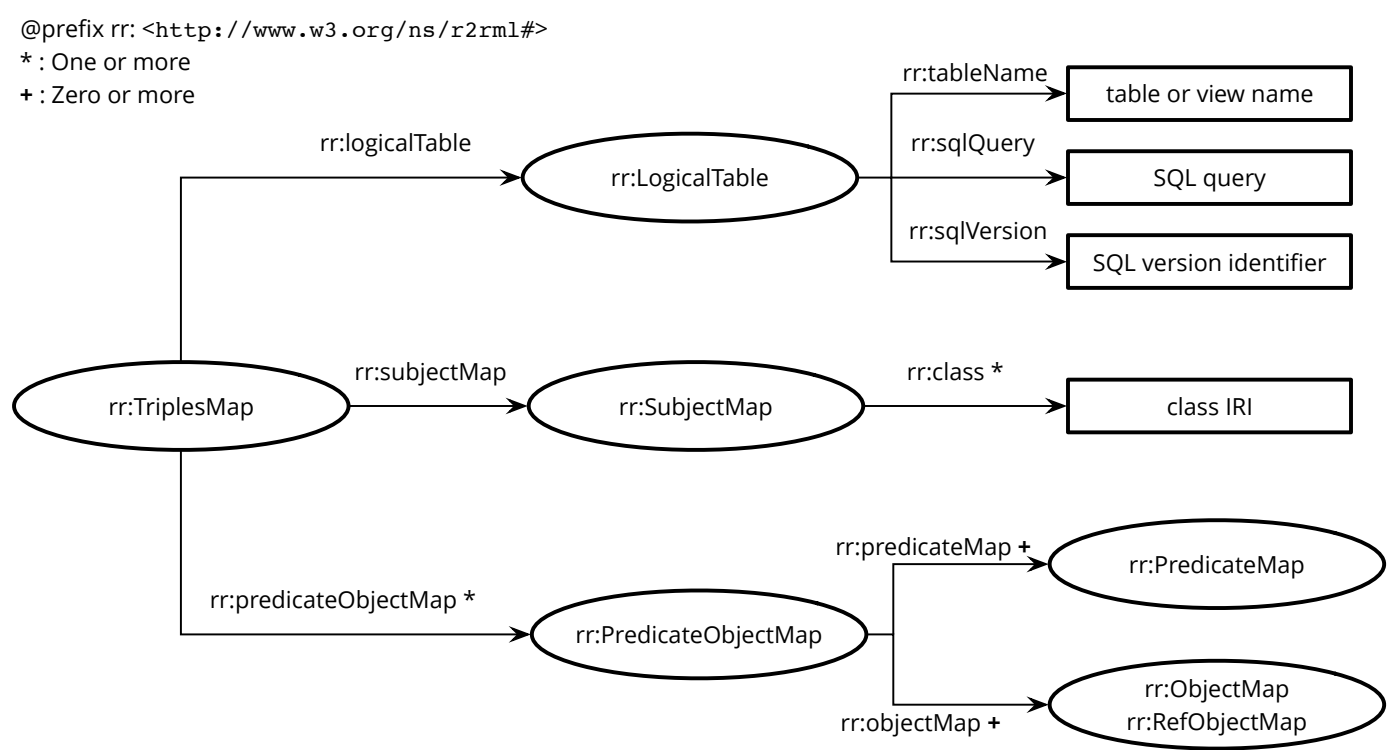

Figure 2.6: R2RML main components (based on Das et al. [2012]).

As shown in Figure 2.6 a Triples Map is defined by:

i) exactly one Logical Table ( $r r$ :LogicalTable), to specify a relational table or view;

ii) exactly one Subject Map ( $r r$ :SubjectMap), to specify the IRIs of the classes generated from the logical table

iii) zero or more Predicate-object Maps ( $r r$ :PredicateObjectMap), that are composed by:

a) one ore many Predicate Maps ( $r$ :PredicateMap)

b) one or many Object Maps ( $r$ : ObjectMap), or Reference Object Maps ( $r$ :PredicateObjectMap) when joins with another logical table are needed. 


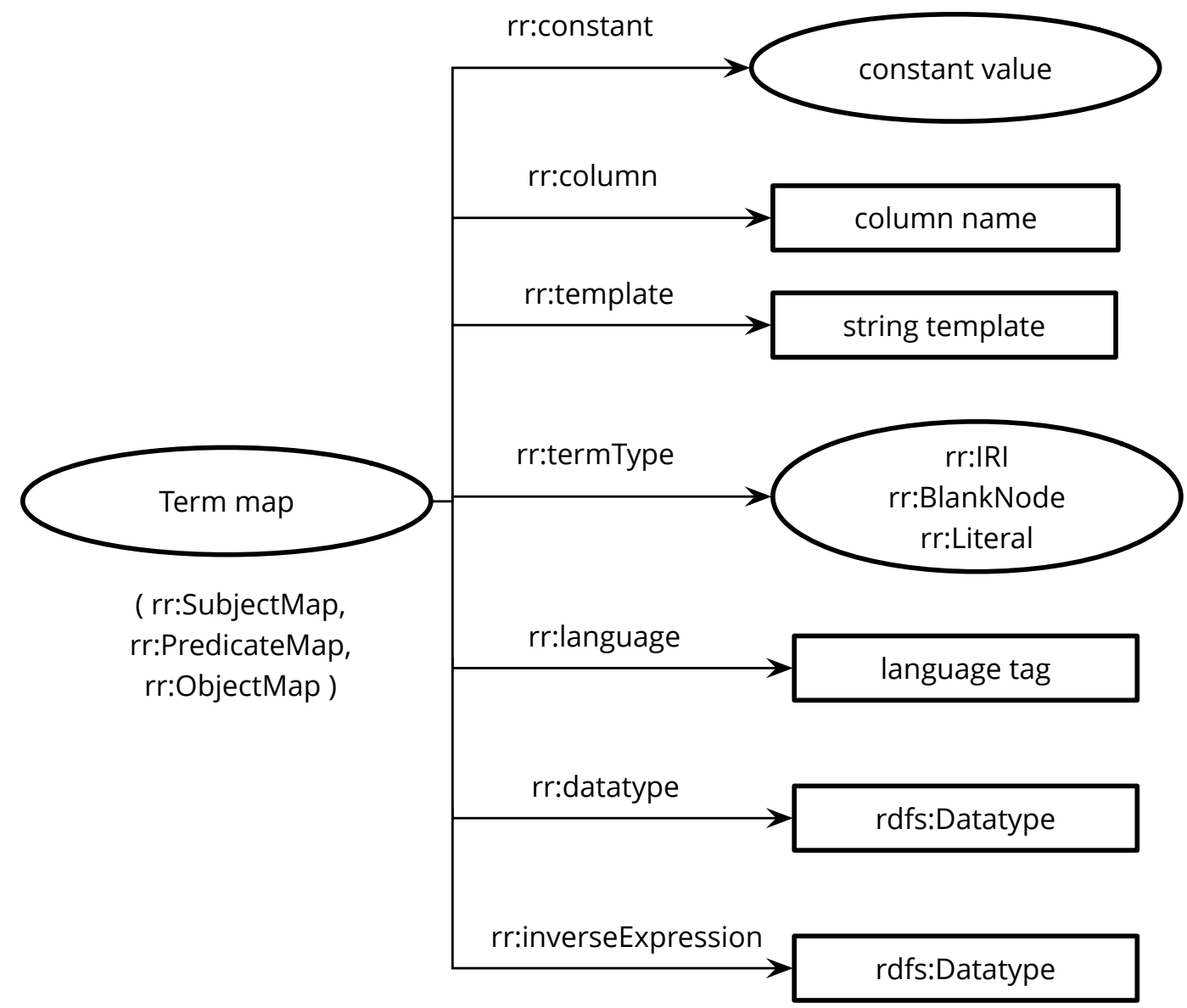

Figure 2.7: R2RML Term maps (based on Das et al. [2012]).

As shown in Figure 2.7, Subject maps, Predicate maps, and Object Maps are Term Maps ( $r$ : TermMap), which are used to generate: $i$ ) IRIs ( $r r:$ IRI) ; ii) Blank Nodes ( $r r$ :BlankNode) ; or iii) Literals ( $r r:$ Literal). The value of a Term Map is specified using Constants ( $r r$ : Constant), Columns ( $r$ : Column), or templates ( $r r$ :Template). A mapping rule can be further specified with Language ( $r r$ : Language), inverse expression ( $r r$ : InverseExpression), and data type ( $r r$ :DataType).

There has been a wide adoption of the standard, both in industry (e.g., Oracle, IBM), and academia (e.g., Morph ${ }^{42}$, ontop ${ }^{43}$ ), with several research works involving the standard. Furthermore, several extensions to R2RML have been proposed to transform data sources beyond relational databases, which indi-

\footnotetext{
${ }^{42}$ https://gi thub.com/oeg-upm/morph-rdb (Last viewed 13th April 2016)

${ }^{43}$ http: //ontop. inf .unibz. it/ (Last viewed 13th April 2016)
} 
cates that the recommendation can be extended for different scenarios and data sources. We describe the most relevant extensions below.

RML - Dimou et al. [2014] proposed the RML generic mapping language, built on top of R2RML to create mapping rules for heterogeneous sources, such as JSON, CSV, or XML. The authors identified three major limitations in the state of the art: i) Mapping of data on a per-source basis ; ii) Mapping of data on a performat basis ; and, iii) Mapping rules reusability.

RML includes a Logical Source class ( $r m l: L o g i c a l S o u r c e)$ that extends the Logical Table class of R2RML to define any type of data source. The Logical Source is described by the following properties:

i) exactly one source ( $\mathrm{rml}$ : source), identified by a URI to locate the data source,

ii) exactly one logical iterator ( $\mathrm{rml}$ : i terator), that defines the iteration mechanism to process the elements in the data source,

iii) zero or one reference formulation ( $r m l$ :referenceFormulation), that defines how to refer to elements of the data source.

Finally, RML adds the property reference ( $r m l$ : reference) which contains an expression that is used in Term Maps to select values from the data source, in the same way as R2RML column property selects columns in a relational database. The reference should conform with the specified Reference Formulation language. We show below an example of an RML document in Turtle that defines two data sources: a JSON and an XML data source:

Listing 2.3: RML examples

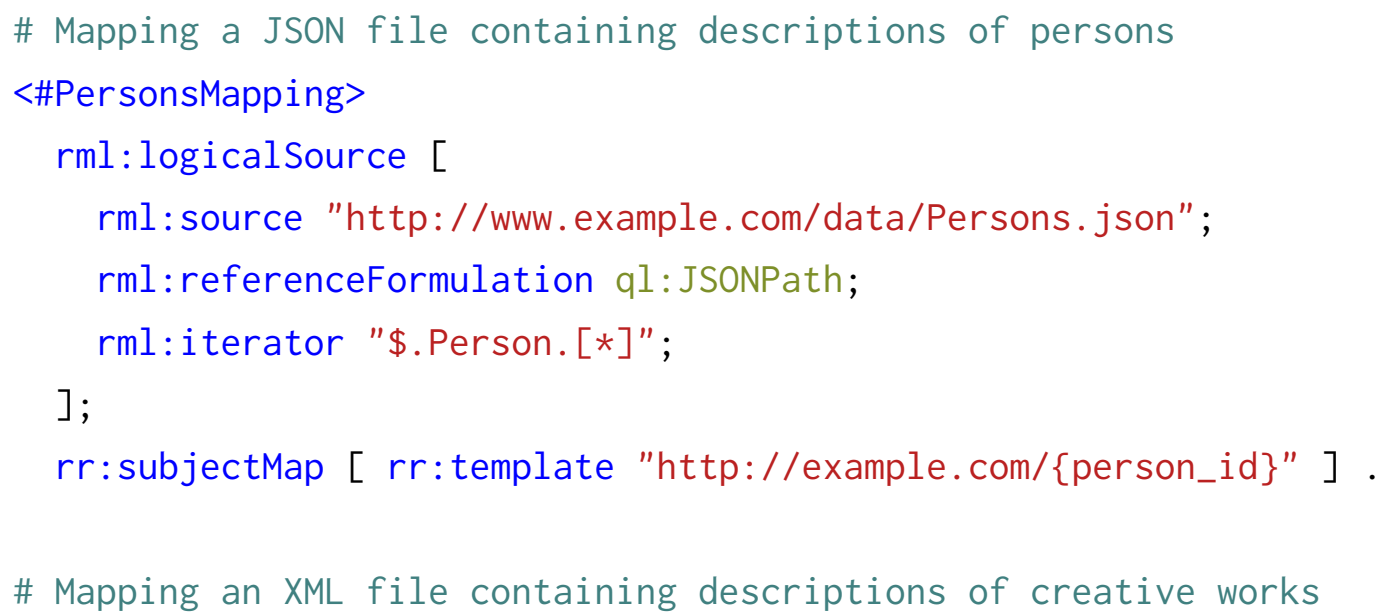




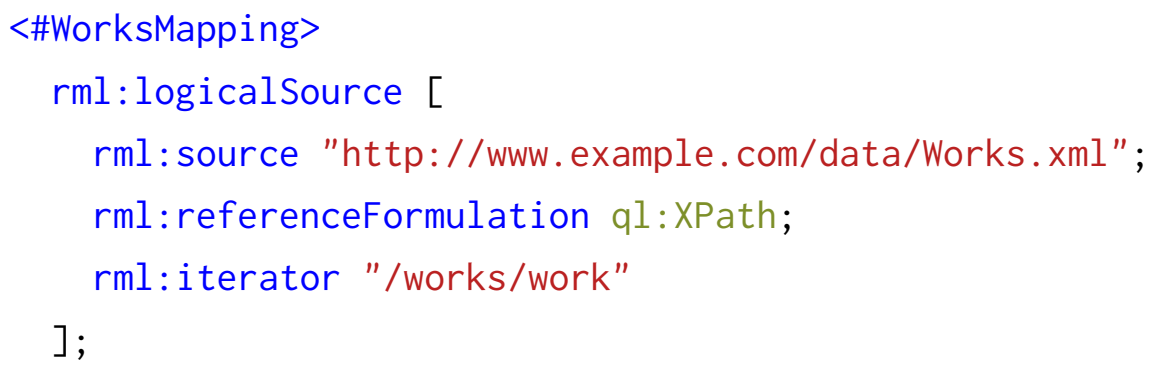

xR2RML - Michel et al. [2015] developed xR2RML, that extends both R2RML and RML to support heterogenous sources with a strong focus on non-relational databases. The authors argue that $\mathrm{R} 2 \mathrm{RML}$ and $\mathrm{RML}$ mappings are tied to specific query languages (e.g., SQL08, JSONPath, etc.). This results in a lack of flexibility to adapt to new types of sources (e.g., key-value stores) without modifying the mapping definitions.

To overcome this limitation and to deal with non-relational databases, $x R 2 R M L$ defines a Logical Source class ( $x r r$ : logicalSource) that extends RML in the following way:

i) it reuses the $\mathrm{RML}$ properties $\mathrm{rml}$ : i terator, and $\mathrm{rml}$ : referenceFormulation.

ii) it drops the rml: source property to avoid being tied to the specific data access mechanisms.

iii) it includes the Query property (xrr:query), that expresses a valid query with regards to the query language of the data source.

Furthermore, $x R 2 R M L$ defines the property $x r r$ : reference as an extension of the RML property $\mathrm{rml}$ : reference, that enables retrieving specific data elements. Moreover, $\mathrm{xR} 2 \mathrm{RML}$ introduces the ability of mapping data elements to nested RDF collections by introducing new values of the $r r$ : termType property:

i) $x r r: R d f L i s t$, that generates a $r d f:$ List.

ii) $x r r$ :RdfSeq, that generates a $r d f$ : Seq.

iii) $x r r: R d f B a g$, that generates a $r d f: B a g$.

iv) $x r r:$ RdfAlt, that generates a $r d f: A l t$.

In this way, a nested term map (xrr: nestedTermMap) can be defined in the context of a term map that produces RDF collections or containers. The nested 
term map allows defining the usual properties for term maps (such as language, or term type), that are applied to each of the elements in the collection or container. Finally, the properties xrr: reference and $r r$ : template can be used within a nested term map to recursively process the corresponding data elements. An example of a mapping using xR2RML in Turtle for a MongoDB ${ }^{44}$ data source is shown below:

Listing 2.4: xR2RML example

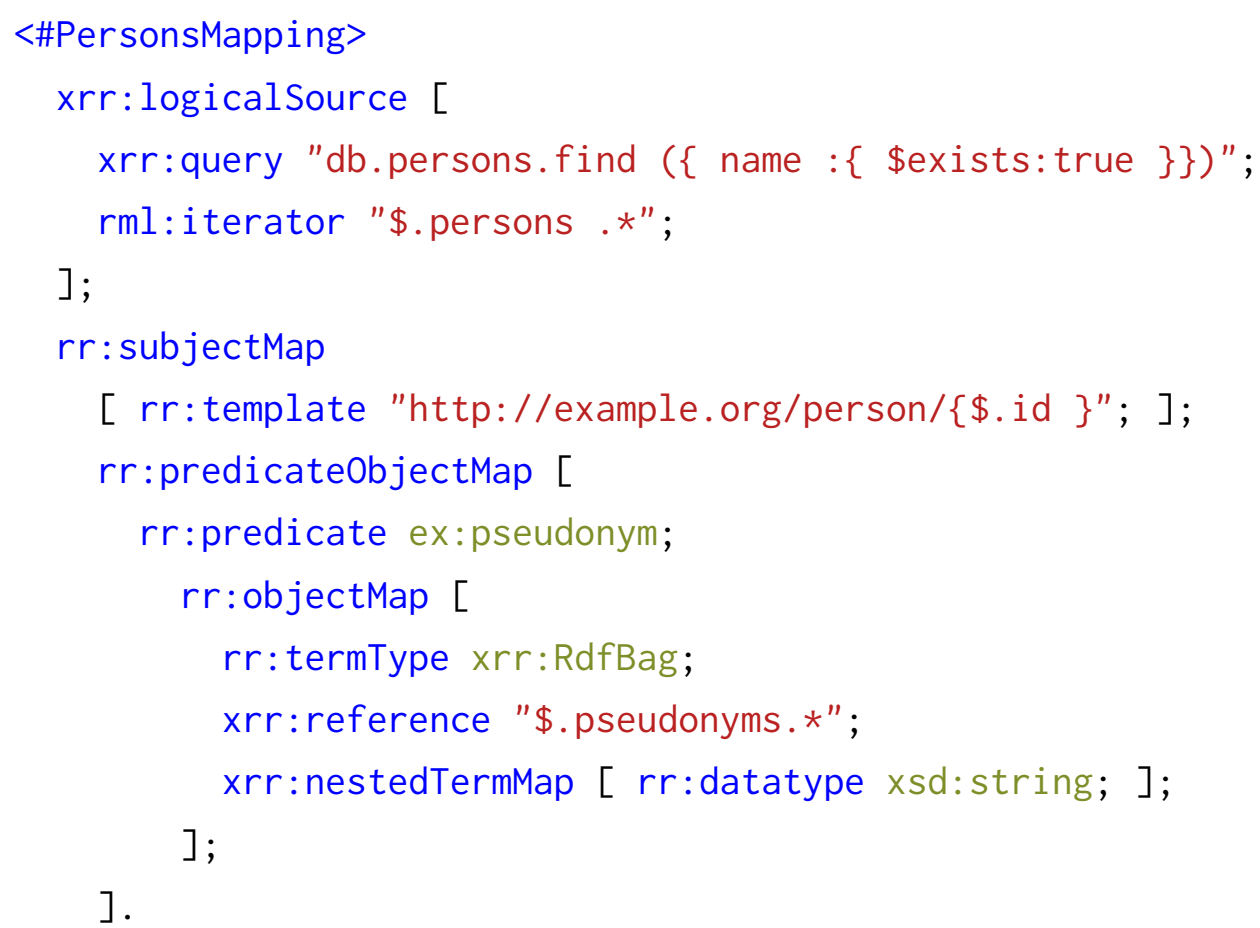

KR2RML - More recently, Slepicka et al. [2015] proposed KR2RML, a mapping language supporting the data integration tool Karma ${ }^{45}$, which extends R2RML to deal with hierarchical data such as that found in JSON or XML objects. The main goal of KR2RML is to provide a source-agnostic mechanism that allows adding new input and output formats without modifying the language or the processor. KR2RML relies on the Nested Relational Model (NRM) proposed by Makinouchi [1977]. Every input data source is translated into NRM form so that every subsequent step (e.g., mapping to RDF, transformation, cleaning) is

\footnotetext{
${ }^{44}$ MongoDB is a document oriented database (https://www.mongodb.com) (Last viewed 13th April 2016)

${ }^{45}$ http: //usc-isi-i2.github.io/karma/ (Last viewed 13th April 2016)
} 
applied to this intermediary form. In this way, adding new input formats only requires a parser and a translator into NRM.

In KR2RML, the data is organized into tables, columns, and rows, where a column in a table can be either a scalar value or a nested table. For example, a column "works" that contains an array of creative works described by their titles can be mapped to a nested table "works". Nested elements can be accessed using a path to the column from the top level table (e.g., ["works", "title"] provides access to a nested table containing the titles of creative works). Differently from Michel et al. [2015] the nested tables can be applied to any data item, not only to items maping to RDF lists or containers.

KR2RML proposes the following re-interpretation of several R2RML language elements:

i) rr: column, besides column-valued term maps (e.g. "first name"), JSON arrays can be used to capture the column names that define the path to a nested column from the document root (e.g., [“works", "title”]).

ii) $r r$ : joinCondition, this element is not allowed for non-relational data due to its impracticability for very large data sources.

In order to apply the KR2RML mapping document to the NRM and generate $\mathrm{RDF}$, the KR2RML processor performs three steps:

(1) Triples Maps are translated into an execution plan by modelling the R2RML mapping as a directed, cyclic multigraph, and removing cycles to create a topological ordering over the Triples Maps.

(2) Triples Maps are evaluated by traversing the NRM.

(3) The output is serialized into RDF.

Finally, in the literature we find several proposals to facilitate the definition of mapping rules by ontological engineers and domain experts. Barrasa et al. [2004] implemented the ODEMapster processor for the $\mathrm{R}_{2} \mathrm{O}$ mapping language, and a Neon plugin that provided a graphical user interface for defining the mapping rules. More recently, Sequeda et al. [2014] proposed an interesting approach to benefit from a set of mapping rules defined by domain experts. Pequeno et al. [2014] proposed a semi-automatic mapping generation process, where R2RML mappings are generated based on a set of semantic correspondences defined by domain experts. Sengupta et al. 
[2013] presented a graphical R2RML mapping editor to help non-technical users to create and modify their mappings. Finally, de Medeiros et al. [2015] proposed MIRROR, an automatic approach to extract R2RML mappings directly from the relational schema of a database, with the goal of bootstrapping the mapping process.

\subsubsection{Discussion}

In this section, we have reviewed different approaches for mapping heterogeneous data sources into ontologies and RDF. In this section, we review the limitations of existing approaches with respect to mapping MARC 21 records into RDF.

Source schema - Mappings and transformation rules are expressed with regards to a source schema. Most of the approaches rely on a relational schema expressed as views, tables, queries, and columns of a database. For relational databases, the R2RML standard has been widely adopted by both industry and academia. Moreover, recent approaches extend or reinterpret R2RML to include other types of sources such as CSV, XML, JSON, or NoSQL databases (Dimou et al. [2014], Michel et al. [2015], Slepicka et al. [2015]), where the notion of schema is less well defined than in the relational database scenario. MARC 21 data sources do not have an explicit schema and therefore fall into this last category. The most flexible approach in this direction is KR2RML by Slepicka et al. [2015] which is loosely based on the Nested Relational Model. However, Slepicka et al. [2015] do not provide a formal framework to represent hierarchical or schemaless data sources and their mapping language is tightly coupled with the Karma Tool.

Mapping and query language - There are two main approaches for the definition of mapping rules. The first approach defines rules in terms of iterators (Dimou et al. [2014], Michel et al. [2015]) over the data, such as JSONPath, ad-hoc NoSQL queries, or XPath. The second approach uses a format-agnostic path based on NRM (Slepicka et al. [2015]). As MARC 21 records are hierarchi$\mathrm{cal}$, there are not standard iteration mechanisms or query language, the most suitable approach is the one proposed by Slepicka et al. [2015]. Nevertheless, KR2RML does not provide any solution for querying and selecting data elements within the NRM and instead relies on User Defined Functions (UDF) and a simple navigation of nested columns based on a JSON array. 
Domain experts support - Besides processing mapping rules, several approaches have focused on supporting domain experts during the definition of mapping rules. These approaches provide graphical user interfaces (e.g., ODEMapster and Karma Data Integration tool), and bootstrapping mechanisms (e.g., MIRROR).

\subsection{Methods for ontology development}

As we have discussed earlier, the existing works for transforming library catalogues into richer models lack principled methods for integrating the feedback of library experts in the transformation and modelling process. As our main objective is to use ontologies to transform library catalogues, in this section, we review the main methodologies and approaches within the ontology engineering field. In this review, we focus on the technical activities and especially on the specification of requirements, ontology reuse and team collaboration mechanisms, which are the most related areas to the scope of our thesis. We organize the review into methodologies, lightweight approaches, a brief introduction to the NeOn methodology, and methods for ontology reuse.

\subsubsection{Methodologies for ontology development}

In this section, we include a brief description of the five major ontology development methodologies proposed in the area of ontological engineering, listed in chronological order.

Grüninger and Fox [1995] proposed an ontology development process that comprised six activities: motivating scenario, informal competency questions, terminology of the ontology, formal competency questions, first-order logic axioms, and completeness theorems.

The first activity involves presenting a motivating scenario to describe the target use case. The second activity requires the specification of informal competency questions that the desired ontology must be able to answer. These competency questions are considered the requirements of the ontology. The third activity consists in specifying the terminology of the ontology based on the competency questions and formalized using first-order-logic. The fourth activity involves the formal definition of competency questions as an entailment with respect to the 
axioms in the ontology. The fifth activity is an iterative process that consists in defining first-order-logic axioms that specify the terms and constraints until the competency questions are fully represented. Finally, the sixth activity consists in evaluating the competency questions by proving completeness theorems.

The most relevant idea from this early methodology is the concept of Competency Questions (CQs), which will be later used in other methodologies for the specification of requirements.

METHONTOLOGY was proposed by Fernández-López et al. [1997] to support the ontology development at the knowledge level. The methodology proposes a life cycle for ontology development based on evolving prototypes so that it is possible to add, change and remove terms in each iteration. METHONTOLOGY also defines the set of activities to be carried out during the ontology development process grouped by management, technical, and support activities. In METHONTOLOGY, the management and support activities are carried out simultaneously with the technical activities. The technical activities of METHONTOLOGY are specification, conceptualization, formalization, implementation, and maintenance. METHONTOLOGY does not provide any specific mechanism for enabling the active participation of domain experts in the technical activities.

On-To-Knowledge proposed by Staab et al. [2001] focuses on the application-driven development of ontologies. The proposed life-cycle consists of five phases: i) feasibility study, that deals with the identification of the problem to be solved; ii) ontology kickoff, that deals with the specification of requirements and the analysis of input sources; iii) refinement; iv) evaluation; $v$ ) and maintenance. On-ToKnowledge proposes an iterative development model with iterations between the refinement and evaluation phases, and between the refinement and maintenance phases. Thus, the methodology does not foresee iterations during the elicitation of requirements and analysis of input sources, which poses a challenge for developing an ontology for library data sources with library experts.

DILIGENT was proposed by Pinto et al. [2004] to support ontology development in a distributed environment, in a loosely-controlled way and considering the evolution of ontologies. In this methodology, the actors involved in the collaborative development of the same ontology are experts with different and complementary skills, ontology users and ontology developers. The general process proposed comprises five main activities: build, local adaptation, analysis, revision, 
and local update. During the build activity, domain experts, users, knowledge engineers and ontology engineers develop an initial shared ontology. In their local environment they are free to change a copy of the shared ontology. However, they are not allowed to directly change the ontology shared by all users. The control board collects change requests to the shared ontology and analyses them in order to decide which changes will be introduced in the next version of the shared ontology. This methodology addresses, at least partially, the participation of domain experts in the development process and the evolution of ontology requirements. However, it does not provide specific mechanisms for the automatic extraction of evolving requirements from data. Moreover, DILIGENT does not address the question of ontology reuse, which is a key requirement in the library domain.

NeOn (Suárez-Figueroa et al. [2015]) is a methodology for ontology networks development. The NeOn methodology is one of the outcomes of the NeOn European project $^{46}$. Its main goal is to provide support for the drawbacks identified in the previous methodologies, namely, to address the collaborative dimension of the development and to provide concrete guidelines for reusing and reengineering knowledge sources. To do so, this methodology identifies nine flexible scenarios for building ontology networks, as it has been realized that there are many alternatives or ways to build ontologies and ontology networks. The methodology includes the knowledge acquisition activities as a support activity that should be carried out during the whole development process. The strong focus on ontology reuse and its flexibility make the $\mathrm{NeOn}$ methodology suitable for the library domain. However, the NeOn methodology lacks the mechanisms for extracting the evolving requirements and for facilitating the direct participation of domain experts in the ontology design process.

\subsubsection{Lightweigth approaches for building ontologies}

In this section, we introduce a brief chronological description of three lightweight approaches to ontology development.

eXtreme Ontology proposed by Hristozova and Sterling [2002] is an iterative and incremental method that aims at developing ontologies by applying the principles and techniques of the agile software methodology eXtreme Programming

\footnotetext{
${ }^{46}$ http://www. neon-project.org/ (Last viewed 13th April 2016)
} 
(Shore and Warden [2007]) The method consists of the following steps: requirement elicitation, defining competency questions, running validation tests, running redundancy tests, planning, integrating, and running acceptance tests. The eXtreme methodology also relies on CQs provided by the users for the elicitation of requirements and does not take into account the ontology reuse activity.

RapidOWL by Auer [2006] is an adaptive, light-weight methodology for collaborative Knowledge Engineering inspired by the wiki approach to collaboration. This methodology is based on the idea of iterative refinement, annotation and structuring of a knowledge base. Based on the core ideas of agile methodologies, RapidOWL defines values from which principles are derived for the engineering process in general, as well as practices for establishing those principles. RapidOWL practices are inspired by eXtreme Programming and include: i) joint ontology design, to ease collaboration between knowledge engineers, domain experts and users; $i$ ) information integration, to ground the knowledge elicitation on existing information; iii) view generation, to provide domain specific views for human users and software systems; and, iv) ontology evolution, for enabling the smooth adoption of models and corresponding instance data migration. These practices tackle two key aspects for the library domain scenario, namely joint ontology design between ontology engineers and domain experts, and information integration to ground the ontology design on real data sources. However, due to the design principles of RapidOWL, these practices are loosely defined.

XD Methodology was first introduced by Suárez-Figueroa et al. [2009] and revisited by Presutti et al. [2012]. The XD methodology is also inspired by the principles and techniques of eXtreme Programming. The main principles of the XD methodology can be summarized as follows: $i$ ) customer involvement and feedback; ii) customer stories, competency questions, and contextual statements; iii) iterative development; iv) test-driven design; $v$ ) modular design; vi) collaboration and integration; vii) task-oriented design; and viii) pair-design.

\subsubsection{The NeOn methodology: An overview}

The NeOn methodology provides support for the ontology engineering process (SuárezFigueroa et al. [2015]). The NeOn methodology is particularly suitable for the library domain as it has a strong focus on reuse and reengineering of ontological resources. 
Suárez-Figueroa et al. [2015] defined an ontology network as a group of ontologies that are linked to each other by a variety of different meta-relationships or domain-independent relationships, such as mappings between them, or versioning relations.

The main components of the methodology are: $i$ ) a glossary of processes and activities; ii) nine scenarios for building ontologies and ontology networks; iii) two ontology network life-cycle models; and $i v)$ methodological guidelines for the most used processes and activities. We briefly describe each of the components below:

Glossary of processes and activities - This component collects the definition of terms involved in the processes and activities of the ontology development. An activity is defined as "an action to be performed including its required input and output". A process is defined as "a group or set of ordered activities". The glossary is composed of definitions and explanations of 59 processes and activities, such as Ontology Alignment, which is a process, and Ontology Matching, which is an activity of the ontology alignment process. In this chapter, we adopt the terms and definitions provided by the glossary.

Scenarios - The methodology identifies nine scenarios for building ontologies and ontology networks. Each scenario is a set of ordered processes and activities. These nine scenarios can be combined in different ways but any combination should include the first scenario. The first scenario, named "From specification to implementation", includes the core development activities. Other scenarios include the reenginnering of non-ontological resources (Scenario 2), or the reuse of existing ontologies (Scenario 3).

Ontology network life-cycle models - The framework offers two development lifecycle models that are inspired by the software engineering field: the waterfall and the iterative-incremental models. On the one hand, the waterfall life-cycle model organizes the development stages as sequential phases. The framework offers five different versions of this model, depending on the number of phases and scenarios. This family of models are suitable for projects with well-defined closed requirements, or for projects that cover a small and well-defined domain. On the other hand, the iterative-incremental model structures the development as a set of iterations. Each iteration is developed according to any version of the waterfall model. The iterative-incremental model is more suitable for projects where the requirements evolve, or involve groups of developers with different profiles. Thus, the iterative-incremental life-cycle model is well-suited for col- 
laboratively building an ontology network in a large, heterogeneous domain like the library domain.

Methodological guidelines - The last component of the framework is a set of prescriptive guidelines to inform users on how to perform the different development processes and activities. These guidelines cover several but not all scenarios, processes, and activities. A guideline is composed of: $i$ ) a guiding card, that provides direct and practical information; $i i)$ a graphical workflow, depicting the expected flow and relations among inputs, outputs, and actors; and iii) examples of use.

As discussed above, the application of the $\mathrm{NeOn}$ methodology is driven by the application of a combination of scenarios. We briefly introduce these scenarios below.

Scenario 1. From specification to implementation. This scenario consists of the core activities involved in any ontology development and is expected to be combined with other scenarios. The scenario is composed by five activities: $i$ ) specification; ii) scheduling; iii) conceptualization; iv) formalization; and, v) implementation.

Scenario 2. Reusing and reengineering non-ontological resources. This scenario is applicable only when non-ontological resources are available. A non-ontological resource is a knowledge resource that describes aspects of a certain domain but has not been previously formalized as an ontology. Examples of such resources are lexica, dictionaries, and thesauri.

Scenario 3. Reusing ontological resources. This scenario applies when the developers can have access to ontological resources that cover at least partially the domain of the ontology to be built. These ontological resources can be reused either as a whole or by selecting specific constructs (e.g., class definitions). To perform this scenario, the $\mathrm{NeOn}$ framework defines the ontology resource reuse process, that entails activities such as searching, assessment, selection, and integration.

Scenario 4. Reusing and reengineering ontological resources. This scenario should be applied when the output of Scenario 3 needs some adjustments and extensions to meet the requirements defined in the ontology specification activity. To support this scenario, the framework provides the ontology resource reengineering process, inspired by the software reengineering process of Byrne [1992]. 
The process consists of three activities applied to ontological resources: $i$ ) reverse engineering; $i i)$ restructuring; and, iii) forward engineering.

Scenario 5. Reusing and merging ontological resources. This scenario applies when in Scenario 3, there are available several possibly overlapping ontological resources that can be aligned and merged during the ontology development process.

Scenario 6. Reusing, merging, and reengineering ontological resources. This scenario should be applied when the output (i.e., the merged ontological resources) of Scenario 5 needs some adjustments and extensions. Its application is anologous to the one described for Scenario 4.

Scenario 7. Reusing ontology design patterns. Ontology design patterns provide modelling solutions to well-known ontology modelling problems (Gangemi [2005]). This scenario is useful during the conceptualization and implementation activities, when the developers face recurring modelling problems that can be approached using ontology design patterns.

Scenario 8. Restructuring ontological resources. This scenario is suitable where the ontological resources to be integrated need to be reorganized. In this scenario, the following activities can be applied: $i$ ) modularization; ii) pruning; iii) extension; and, iv) specialization.

Scenario 9. Localizing ontological resources. This scenario applies in cases where the ontological elements of the output ontology network need to be described in different natural languages.

\subsubsection{Methods for ontology reuse}

As highlighted in the previous section, reusing widely adopted ontologies can reduce the time and effort required for developing ontologies. One of the open questions in ontology reuse is how to help users to find the appropriate terms and ontologies for a certain application or domain of interest. In this section, we review relevant works related to this question. In particular, we review the works related to ontology search and the related works on ontology similarity as are the main core techniques used in ontology reuse so far. 


\section{Ontology search}

Allocca et al. [2011] argued that a key aspect for users searching and browsing ontology repositories lies in the ability of the system to show the relationships between ontologies, and more specifically some relationships that are not found in the ontology statements (i.e., not explicit statements via imports, reuse of terms, etc.) such as their similarity to other ontologies in terms of the domain they model or the topics they cover (i.e., thematic relationships).

Allocca et al. [2011] introduced the DOOR ontology as an attempt to describe different types of relation among ontologies. The main goal was to facilitate the explicit description of the relationships that were previously implicit. With respect to overlapping ontologies, the authors defined the lexicographically similar to relation which is defined as how an ontology overlap/cover parts of the same area of interest of another ontology by directly measuring the overlapping of terms among two ontologies.

Allocca et al. [2012] later hypothesized that some of the difficulties of searching ontologies are related to the information about implicit relationships between ontologies in search results. To authors studied the impact of grouping ontology search results by six types of relationships: comes from the same domain, similar to, is previous version of, is syntactically similar to and is included in. To this end, the authors carried out a user-centered evaluation showing that the most useful ontology relationships for users were comes from the same domain and similar to. These experimental results pointed at the importance of thematic relationships and sophisticated similarity measures. In the light of these results, the authors highlighted the need of sophisticated mechanisms to automatically extract this type of relationships.

Schaible et al. [2014] conducted a study to investigate the current practice of vocabulary reuse during linked data modelling. In their study, the authors identified three different aspects of the decision process when reusing vocabularies, namely: $i)$ reusing vocabularies versus creating new terms and linking them to other vocabularies, ii) appropriate mix of vocabularies, and iii) usefulness of vocabulary metadata. Regarding metadata, the survey results showed that the information on the number of data sets reusing the vocabulary and the information on the domain of the vocabulary are the preferred pieces of information. In particular, a combination of both obtained the best results in the study. There are already several 
methods to gather approximate information on the popularity of a vocabulary such as the LOV repository ${ }^{47}$ or LODstats. ${ }^{48}$ However, there is a lack of mechanisms to (semi-)automatically gather topical information of vocabularies.

Vandenbussche and Vatant [2014] developed the Linked Open Vocabularies (LOV) repository to facilitate finding vocabularies for their reuse in the context of linked data. The most interesting aspect of LOV is their curation workflow, assisted by human editors, to guarantee a certain degree of quality of the vocabularies made available through the repository. Regarding the search capabilities, LOV search is based on tf-idf and a basic relevance mechanism based on the popularity of the vocabularies. In LOV, the popularity of a vocabulary is based on the number of data sets that reuse it in the LOD cloud ${ }^{49}$. Regarding thematic relationships among the vocabularies, LOV offers a set of categories or tags that have been assigned by humans.

\section{Ontology similarity and relatedness}

Ding et al. [2005] proposed several algorithms for ranking semantic web ontologies to promote reuse. Their algorithms were purely based on explicit reference or links between the ontologies, and no textual information was taken into account.

David and Euzenat [2008] compared several ontology distances to calculate the similarity between ontologies. The metrics studied were either based on the structure of the ontologies or on string similarity metrics applied to their lexical elements. In later work, they proposed metrics exclusively based in the alignments between ontologies (David and Euzenat [2008]).

Cheng and $\mathrm{Qu}$ [2013] proposed a classification for vocabulary relatedness on the Web of Data and performed an empirical study with more than 2,000 vocabularies using graph analysis techniques. Their classification differentiates three kinds of relatedness: $i$ ) declarative relatedness, which was related to the structural properties contained in the ontology statements such as imports or equivalences, $i i$ ) topical relatedness, which measured the overlap of the topics reflected in the textual descriptions of vocabularies and iii) distributional relatedness, which com-

\footnotetext{
${ }^{47}$ http: //lov.okfn.org/dataset/lov (Last viewed 13th April 2016)

${ }^{48}$ http: //stats. lod2.eu/ (Last viewed 13th April 2016)

${ }^{49}$ This information is gathered from the LODstats data set
} 
pared lexical descriptions of each pair of terms of the same type (i.e., a class is compared only with another class) using a string metric for ontology alignment (Stoilos et al. [2005]). Textual descriptions were gathered from $i$ ) labels of terms (e.g., rdfs:label, dc:title, and 84 inferred sub-properties), and ii) the term IRI local name, $i i$ ), The results of the analysis indicated that many topically related vocabularies are either independent copies of the same underlying conceptualization or developed by different publishers to describe a common domain.

\subsubsection{Discussion}

In this section, we have reviewed the main methodologies and approaches for ontology development. In this section, we summarize their main limitations with respect to the development of library ontologies. We organize the discussion into three key features for the library domain: knowledge acquisition and requirements elicitation, collaboration of domain experts, and ontology reuse.

Knowledge acquisition and requirement elicitation - Starting with Grüninger and Fox [1995], most of the approaches rely on competency questions for the requirements elicitation (Fernández-López et al. [1997], Staab et al. [2001], SuárezFigueroa et al. [2015], Hristozova and Sterling [2002], Presutti et al. [2012]). However, none of the approaches provides explicit guidelines or mechanisms to facilitate the extraction of evolving competency questions and requirements. Moreover, only the $\mathrm{NeOn}$ methodology identifies the knowledge acquisition activity as a key support activity that spans through the whole ontology development process.

Active participation of domain experts - Several approaches and methodologies highlight that the collaboration of domain experts is a critical aspect of ontology development (Suárez-Figueroa et al. [2015], Pinto et al. [2004], Hristozova and Sterling [2002], Auer [2006]). However, none of the approaches provides specific support for enabling the active collaboration between domain experts and ontological engineers through informative patterns extracted from data.

Ontology reuse - The majority of approaches assume that ontologies are built from scratch and do not take into account the scenarios where reusable ontologies already exist. Only the $\mathrm{NeOn}$ methodology provides concrete guidelines and mechanisms for ontology reuse. Regarding the methods for ontology search 
and reuse, most of the works in the literature have focused on: $i$ ) structural relationships such as imports or equivalentClass statements; and $i i)$ string similary and term-based measures, such as tf-idf (Salton and Michael [1983]).

\subsection{Definitions}

To conclude this chapter, we introduce the definitions of the core concepts that will be used throughout the thesis. First, following Gutierrez et al. [2004], we assume there exist pairwise disjoint infinite sets $I, B$, and $L$ (IRIs, Blank nodes, and Literals):

Definition 2.5.1 (RDF triple) A triple $(s, p, o) \in(I \cup B) \times I \times(I \cup B \cup L)$ is called an RDF triple. In this tuple, $s$ is the subject, $p$ is the predicate, and $o$ is the object.

Definition 2.5.2 (RDF graph) An RDF graph $\mathcal{G}$ is a finite set of $R D F$ triples.

Second, based on the MARC21 standard introduced in Chapter 2, assume there are pairwise disjoint finite set $C F$ and $D F$ (Control fields, Data fields):

Definition 2.5.3 (Record) A record $r=(l, F)$ is defined by a leader $l$ and a non empty set offields $F=\left(f_{1}, \ldots, f_{n}\right)$ and $F \in(C F \cup D F)$.

Definition 2.5.4 (Control field) A control field $c f=(t, v), \forall c f \in C F$. In this tuple, $t$ is the field tag, and $v$ is the value of the field.

Definition 2.5.5 (Data field) A data field $d f=(t, i, S F), \forall d f \in D F$. In this tuple, $t$ is the field tag, $i$ is the indicator, and $S F=\left(s f_{1}, \ldots, s f_{p}\right)$ is a non empty set of subfields.

Definition 2.5.6 (Subfield) A subfield $s f=(t, v), \forall s f \in S F$. In this tuple, $t$ is the subfield tag, and $v$ is the value of the subfield.

Definition 2.5.7 (catalogue) A catalogue $\mathcal{C}$ is a finite set of records. 



\section{Hypotheses and objectives}

In this chapter we define the objectives and scope of the work presented in this thesis. First, based on the discussion presented in Chapter 2, we ellaborate on the open research problems and their related research questions. Second, we formulate our main hypotheses under a set of assumptions and limitations. Taking these hypotheses into account, we introduce our research methodology and main objectives. Finally, we introduce the conceptual, methodological and technological contributions of our work.

\subsection{Open research questions}

This work aims at providing theoretical foundations and technical solutions for transforming and publishing library data sources using ontologies and semantic technologies. From the analysis presented in Chapter 2, we have identified a series of open research challenges that can be summarized into the following research problems:

P1. The formal definition of interoperable mapping rules using well-grounded languages and formal frameworks.

P2. Systematic analysis and modelling of library data sources using ontologies to enrich their semantics.

P3. The study of the impact of ontology-based library data access in library applications.

For the first open research problem (P1), regarding the formalization and definition of interoperable mapping rules, we tackle in this thesis the following research question: 
- Can we develop extensions to standard mapping and query languages that are suitable for transforming library catalogue data into RDF? The data integration, ontologybased data access and databases research fields have long studied the mapping and transformation problem. By leveraging constructs and models from these fields, we aim at developing a formal framework for mapping library catalogues into RDF using ontologies.

For the second open research problem (P2), concerning the analysis and modelling of library data sources using ontologies, the research questions that we address in this thesis can be formulated as follows:

- How can we extract patterns from catalogue data to improve the library modelling process? We aim at developing a set of methods and techniques that are able to extract patterns from catalogue data and represent them so that they can be used to improve the library data modelling process.

- How can we support the participation of library experts in the mapping and ontology development processes? Several well-established ontology development methodologies are available. We aim at extending their ability to integrate the feedback from domain experts during the knowledge acquisition activity using the patterns extracted from the catalogue.

- Can we develop methods that help to better understand the thematic similarities between library ontologies? Currently, there are available a wide range of potentially overlapping library ontologies. Moreover, current search engines provide limited support to understand the similarities between existing ontologies. We aim at designing and developing methods that go beyond classical information retrieval methods with the goal of providing more sophisticated mechanisms for comparing and analyzing library ontologies.

Finally, for the third open research problem (P3), regarding the impact of semantic technologies in library end-user applications, in this thesis we deal with the following research question:

- Does the application of semantic technologies to library applications improve the overall experience of end-users? By building a system that applies the methods, models, and constructs developed in this thesis, we aim at measuring their impact on end-user library applications according to different information retrieval performance and usability measures. 


\subsection{Hypotheses}

In this thesis, we formulate five hypotheses that follow from the research problems and that aim at providing answers to the open research questions discussed in the previous section. For the first open research problem (P1), concerned with the definition of interoperable mapping rules, we identify the following hypotheses:

H1. The structure of MARC 21 records can be fully represented using a nested relational model.

H2. A mapping language with minimal modifications to the W3C R2RML can be designed to define mapping rules between MARC 21 data sources and RDF.

For the second open research problem (P2), we propose the evaluation of the following hypotheses:

H3. Analytical data and the feedback of library experts can be used for mapping library catalogues into RDF to develop a library ontology with sufficient quality with respect to state of the art quality metrics.

H4. Probabilistic topic modelling techniques can produce coherent descriptions of ontologies and can perform better than existing methods used in ontology search.

Finally, regarding the impact of semantic technologies on end-user library applications, the third research problem (P3), we aim at testing the following hypothesis:

H5. The application of semantic technologies to end-user library application can result in significantly better user satisfaction and efficiency in completing information retrieval tasks, with respect to current end-user library applications.

\subsection{Assumptions and restrictions}

Our work hypotheses are evaluated considering the following assumptions:

A1. We assume that bibliographic and authority records are available and accessible.

A2. We assume the participation of library experts in the mapping and ontology development processes. 
A3. Our ontology development and mapping methods assume that there are library ontologies available to be reused.

Finally, there is a set of restrictions that defines the limits of our contribution and establishes future research objectives. These restrictions are the following:

R1. The source catalog data conforms to the MARC 21 standard.

R2. The records are described in the MARC 21 Bibliographic and Authority formats.

R3. Our methods focus exclusively on metadata describing library resources. Our methods do not use any content such as the full text of books, images, or videos.

R4. The mapping and transformation methods are executed as batch processes.

\subsection{Research methodology}

This thesis follows the design-science method proposed by von Alan et al. [2004]. The design-science research method focuses on extending the boundaries of human and organizational capabilities by building new and innovative artifacts.

Artifacts are broadly defined as constructs, models, methods, and instantiations. In the design-science method, constructs provide the vocabulary and symbols used to define problems and solutions and enable the construction of models or representations of a specific domain. Methods define processes, typically in the form of algorithms and practices, to find a solution for the problem within the domain. Instatiations, typically in the form of prototypes, show that constructs, models, and methods can be implemented in a working system.

Specifically, in this thesis, we design and build an artifact for transforming and consuming library data sources in the context of the Web of Data. We call this artifact the marimba framework. The marimba framework encompasses a set of constructs, models, methods, and instantiations that are designed and implemented to evaluate the hypotheses presented in Section 3.2. In particular:

- We develop several methods to analyze MARC 21 library catalogues, to assist library experts in mapping and transforming library catalogues into RDF using ontologies.

- We design several constructs and models. First, we propose a formal framework for representing library catalogues using a nested relational model. Second, we 
propose a query language for querying nested relational data. Third, we propose a mapping language that combines the relational model and the query language.

- We implement an instantiation of our proposal, which is composed of several software components corresponding to the different methods, constructs, and models.

\subsection{Main contributions}

In this section, we specify and describe the marimba framework, an ontology-based library data acccess framework. These components are aligned with the main contributions of this thesis. Figure 3.1 provides a high level overview of the marimba framework.

\subsubsection{Constructs and models}

We design constructs and models supporting the transformation and consumption of library data sources on the Web of Data. These constructs and models are related to our hypotheses $\mathrm{H} 1$ and $\mathrm{H} 2$, which we describe as follows.

marimba-datamodel. First, we define an nested relational model for representing library catalogue data.

marimba-sql. Second, we define a language to query data represented in the proposed data model.

marimba-rml. Third, we design a mapping language that can be used to map existing library catalogues into RDF using ontologies.

\subsubsection{Methods and instantiations}

In this thesis, we define and develop several methods and their instantiations.

marimba-mapping. As a first step, we define a method to transform library information sources using ontologies. This method focuses on three key objectives: $i$ ) to provide built-in analytical mechanisms based on catalogue data; ii) to support library experts in mapping existing library data sources into RDF; and iii) to enable the construction of the ontological framework during the mapping process. The proposed method builds on the definition of two methods. First, 


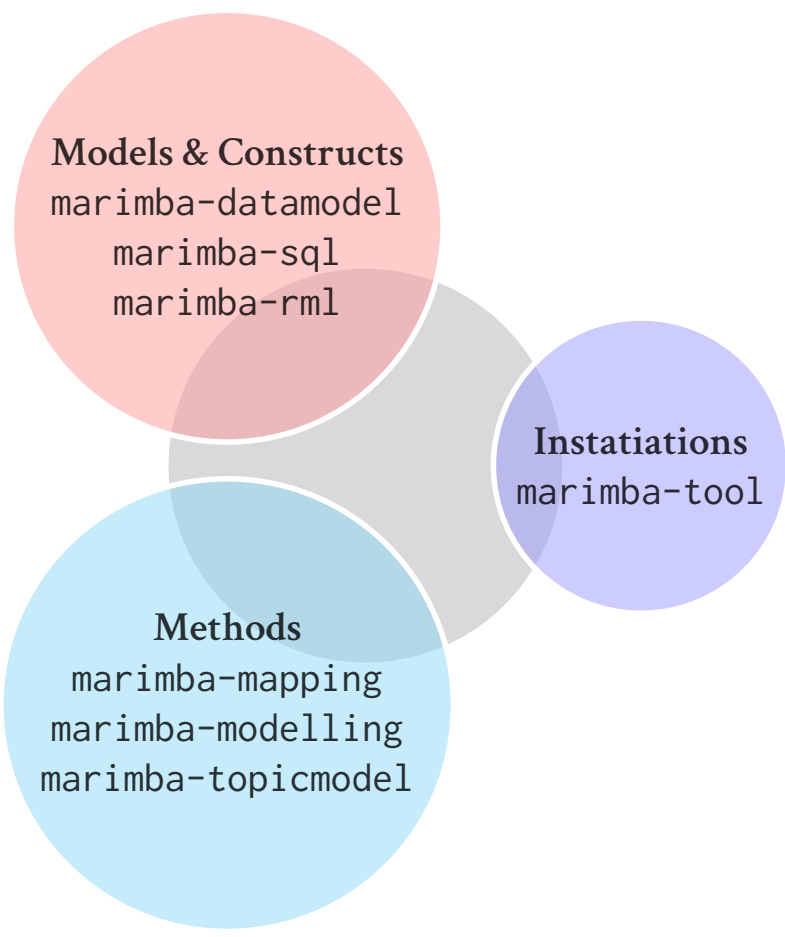

Figure 3.1: The marimba framework and its components

we define a method for extracting and managing mapping templates from existing library data sources. This method enables the representation of mappings in two ways: $i$ ) a human-oriented representation to enable the participation of library experts in the mapping process; and ii) a machine-readable representation, using the marimba-rml and marimba-sql languages.

marimba-modelling. We define a method for semi-automatically supporting the design of the ontological framework during the mapping process. This method supports the library experts in the ontology design process. This method combined with the methods of marimba-mapping is used to evaluate our third hypothesis (H3).

marimba-topicmodel. We design and develop a method to extract the thematic structure of a corpus of ontologies. This method is based on a technique called "probabilistic topic modelling" and provides mechanism for calculating topic-based ontology similarity measures. This method supports the domain experts in the ontology design process process. The method is used to evaluate our fourth hypothesis (H4). 
marimba-tool. We develop an instantiation of the above-described methods and use it to produce a large-scale library data network and a service based on this data network: datos.bne.es. We use this service to assess whether our artifact is able to solve real-world problems, and to test our last hypothesis (H5).

\subsection{Research objectives and outcomes}

Based on the hypotheses and the research method, we organize our work into a set of research and technological objectives. In Table 3.1 we provide a summary of our objectives, contributions, hypotheses, and their evaluation. Our objectives together with the main outcomes are described below:

Research objective 1(RO1). Define an nested relational model to represent library catalog data in a principled way. This research objective is aimed at evaluating our first hypothesis (H1). We perform an analytical study of the proposed nested relational model for representing existing MARC 21 data.

Outcome: marimba-datamodel.

Research objective 2 (RO2). Define a query language and a mapping language to formally represent the mappings of catalogue sources into ontologies. This research objective is aimed at evaluating our second hypothesis (H2). We perform an analytical study, define the syntax and provide the operational semantics of our mapping language.

Outcomes: marimba-sql and marimba-rml.

Research objective 3(RO3). Define the methods for the transformation of library data sources into RDF using ontologies. These two methods allow domain experts to incrementally map existing sources to ontologies, thus participating in the ontological engineering process. This research objective is aimed at evaluating our third hypothesis (H3). We evaluate this hypothesis by: $i$ ) transforming a large and heterogeneous library catalogue; $i i)$ designing an ontology network with a team of library experts to model the catalogue data of the National Library of Spain; iii) performing a topology-based evaluation using state of the art quality metrics; and, iv) performing two user-centered experiment with a large group of users. 
Outcomes: marimba-mapping and marimba-modelling.

Research objective 4(RO4). Define a method for representing ontologies as a mixture of topics in order to facilitate their comparison with other ontologies. This method provides a novel way to support library experts in the selection of ontological terms during the process defined in RO1 using topic-based ontology similarity measures. This research objective is aimed at evaluating our fourth hypothesis (H4) by: $i$ ) measuring the coherence of the produced topics with stateof-the-art coherence measures; and, $i i)$ comparing it to existing approaches on a task-based experiment for ontology clustering.

Outcomes: marimba-topicmodel.

Technological objective 1 (TO1). Develop a system that implements the framework covering the components and processes defined by RO1. This implemented system integrates the different research objectives and is able to process and produce large-scale library data sets. This tool is used to support the evaluation of our first (H3) and fifth hypotheses (H5).

Outcome: marimba-tool.

Technological objective 2 (TO2). Develop different techniques for topic modeling in ontologies that can be used for selection of candidate ontologies during the ontological engineering process. This implementation covers the research objective $\mathrm{RO} 2$ and is used to evaluate our fourth hypothesis (H4).

Outcome: marimba-topicmodel implementation.

In the next section, we provide a brief introduction to our proposed framework to put in context the contributions of the thesis.

\subsection{Marimba framework overview}

In the context of this thesis, we design and build the marimba-framework, an ontologybased library data access framework. The marimba-framework encompasses four core activities: schema extraction, mapping templates generation, ontology development, and RDF generation. Figure 3.2 depicts our main contributions and the flow of activities within the marimba framework. The main input is the catalogue data to be transformed and the output is the transformed catalogue into an RDF graph. We 
3.7. Marimba framework overview

\begin{tabular}{|c|c|c|c|c|c|c|}
\hline 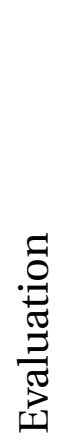 & 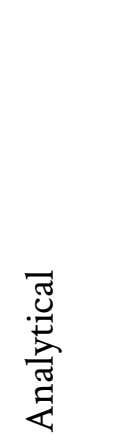 & 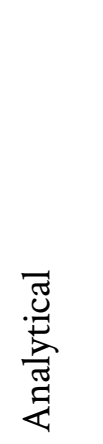 & 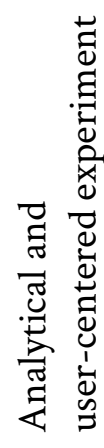 & 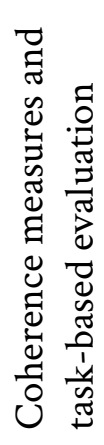 & 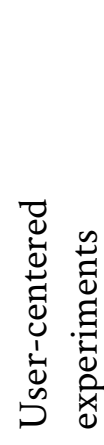 & 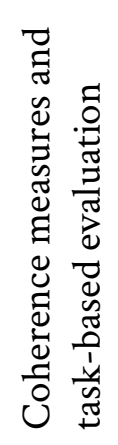 \\
\hline 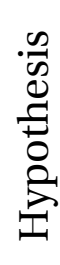 & $\bar{I}$ & $\underline{I}$ & 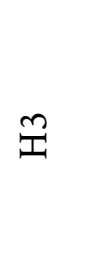 & 竎 & 全 & 空 \\
\hline 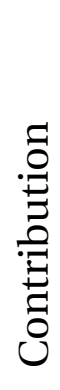 & 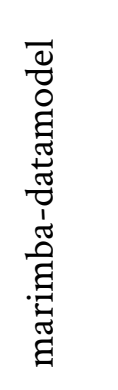 & 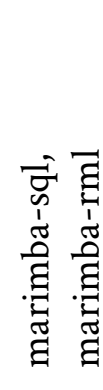 & 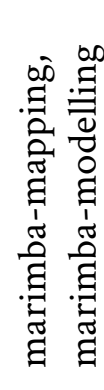 & 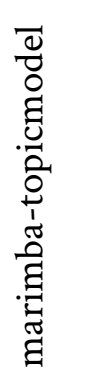 & 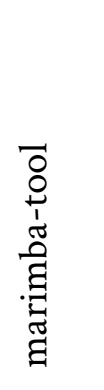 & 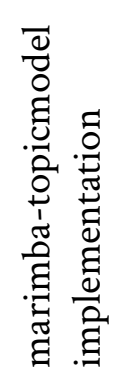 \\
\hline : & 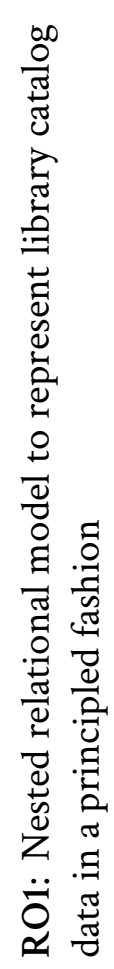 & 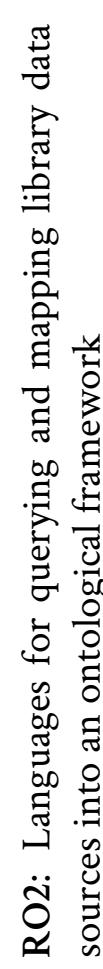 & 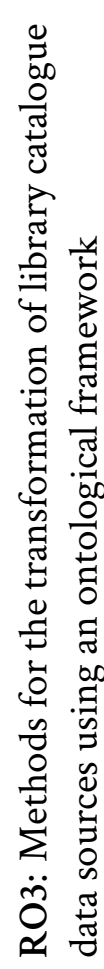 & 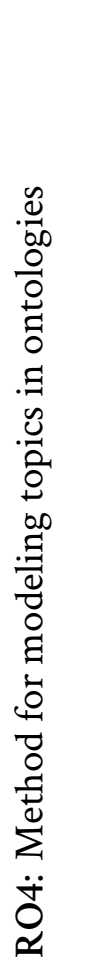 & 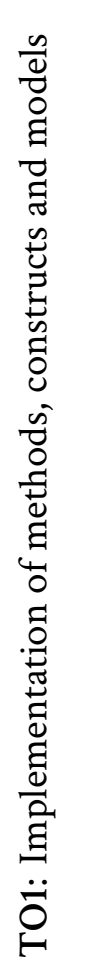 & 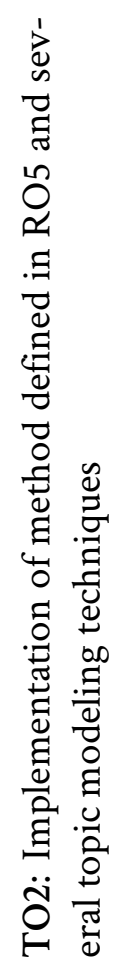 \\
\hline
\end{tabular}


introduce each of the activities and indicate their relation to the main contributions below.

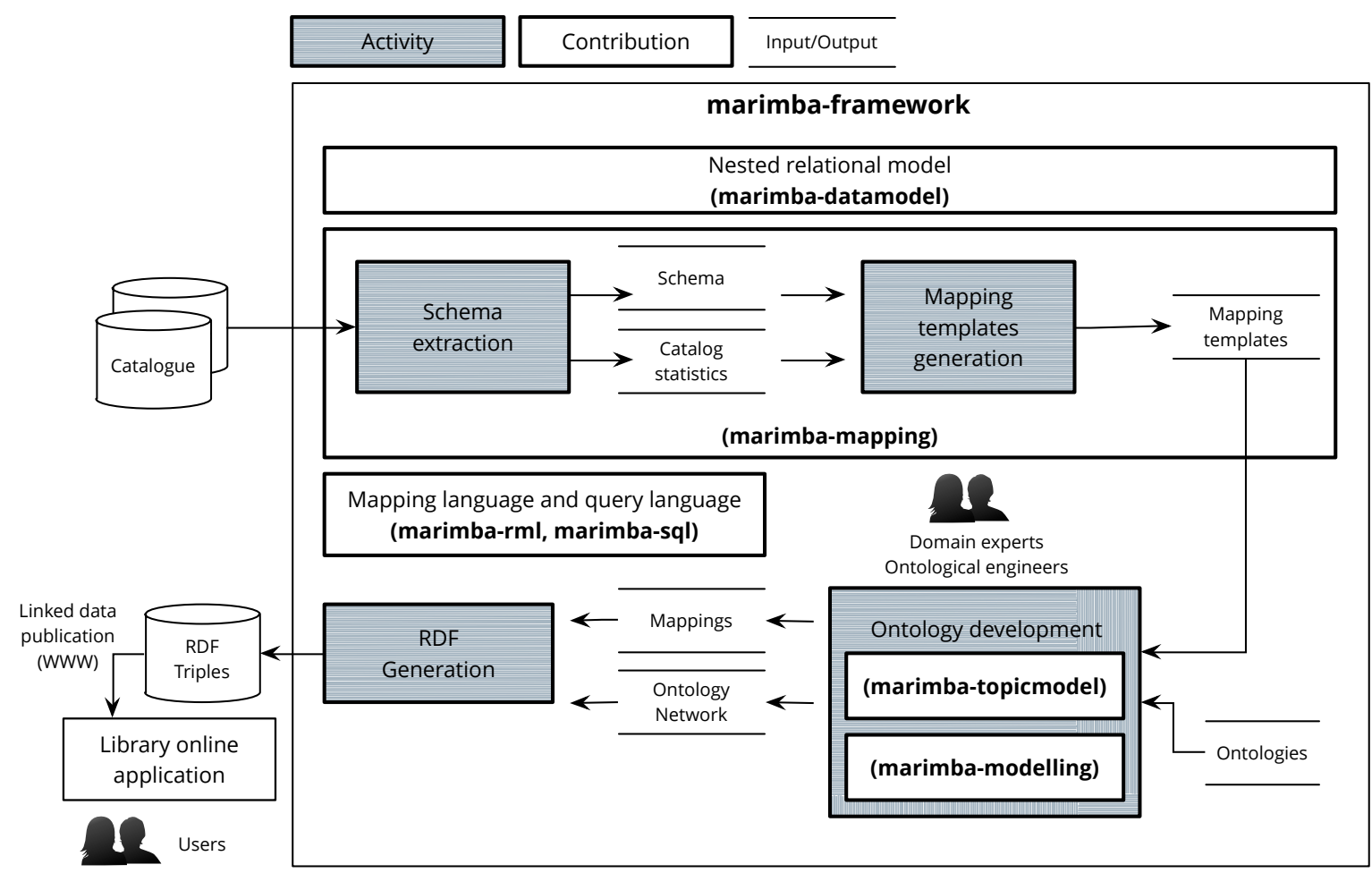

Figure 3.2: Overview of our framework

Schema extraction - This activity uses a novel schema extraction method for analyzing the source catalogue and providing structured information to define the mapping rules. Catalogue data is analized under the formal framework defined by the marimba-datamodel. The schema extraction method is part of our contribution marimba-mapping, which we define and discuss in Chapter 4.

Mapping templates generation - This activity utilizes the schema extracted from the source catalogue to create templates that provide structured feedback to experts participating in the mapping process. This activity uses the extracted schema to present the metadata usage patterns in a way that is useful for experts defining the mapping rules and designing a library ontology. This method is part of our contribution marimba-mapping. We detail this method in Chapter 4. 
Ontology development - This activity corresponds to the construction of an ontology network for transforming library catalogues into RDF. This activity allows experts to reuse several ontological resources during the definition of mapping rules. The activity deals with the methodological aspects of our contribution marimba-modelling and is used to evaluate our third hypothesis (H3). We detail this activity and propose a life-cycle model in Chapter 5. Also, in Chapter 6, we present a method for extracting topics from ontologies and two topic-based ontology similarity measures, which can be used to compare overlapping ontologies (marimba-topicmodel). This topic modelling method is used to evaluate our fourth hypothesis (H4).

RDF Generation - This activity processes the mapping rules and transforms the catalogue into RDF. This activity is discussed in Chapter 7. This component builds on our proposed mapping and query languages (marimba-rml and marimbasql respectively), which are related to hypotheses $\mathrm{H} 1$ and $\mathrm{H} 2$. The marimba-rml and marimba-sql languages are described in Chapter 4.

As mentioned in Section 6.2, the constructs, models, and methods have been implemented as two instantiations: marimba-tool and marimba-topicmodel-tool. These instantiations are used to further evaluate the hypotheses and in particular, our fifth hypothesis (H5) regarding the positive impact of semantic technologies on end-user library applications.

\subsection{Summary}

In this chapter, we have defined our hypotheses and research objectives. Using the definitions provided in Section 2.5, the broad goal of our framework is to transform a catalogue $\mathcal{C}$ into an RDF graph $\mathcal{G}$. Specifically, for each record $r \in \mathcal{C}$, our framework generates a set of RDF triples $\mathcal{T}$. In order to generate RDF triples from a record, we define a set of mapping rules using the formal languages that will be described in Chapter 4. The ontology network used to define the RDF triples in $\mathcal{G}$ is developed through an ontological engineering life-cycle model that is described in Chapter 5. In Chapter 6, we propose a method for extracting latent topics from ontologies and a set of related topic-based ontology similarity measures. In Chapter 7, we discuss the application of our framework to a real application. Finally, we present a user-centered evaluation of this application in Chapter 8. 

As discussed in Section 2.2, mapping MARC 21 catalogues into RDF is a challenging problem. First, MARC 21 records present a nested structure based on records, fields, indicators, and subfields, what makes it difficult to use existing approaches such as mapping languages for relational databases. Moreover, some fields and subfields can contain more than one value. Second, although MARC 21 records are described according to rules and using standard metadata elements, data sources in MARC 21 do not follow an explicit schema and the use of metadata standards varies from one catalogue to another. Finally, unlike other types of data such as relational databases, XML or JSON, there are no standardized methods to query or iterate over MARC 21 records. These characteristics motivate the design, implementation, and evaluation of specific constructs, models and methods to enable and support the mapping processes that will be presented in this chapter.

To this end, the main focus of the chapter is our first research problem (P1), namely the formal definition of interoperable mapping rules using well-grounded languages and formal frameworks. In the next section, we introduce our contribution to this problem, marimba-mapping, and the structure of the chapter.

\subsection{Contributions}

In this section, we introduce our proposal for mapping MARC 21 catalogues into RDF using ontologies. Our proposal is motivated by the limitations introduced in Sections 2.2.1 and 2.3.1, namely: $i$ ) the lack of languages for defining the mapping rules, $i i)$ the lack of schema of MARC 21 data sources, and iii) the lack of support for library experts. To overcome these limitations, we propose marimba-mapping, 
which emcompasses several methods, models and constructs. We present an overview of the marimba-mapping in Figure 4.1 and detail the its components below.

\begin{tabular}{|c|c|}
\hline \multicolumn{2}{|c|}{ marimba-mapping } \\
\hline \multirow[t]{2}{*}{ Construct/Model } & marimba-rml processor \\
\hline & marimba-rml \\
\hline Mapping template generation & marimba-sql \\
\hline Schema extraction & Recursive relational algebra \\
\hline \multicolumn{2}{|c|}{ marimba-datamodel } \\
\hline
\end{tabular}

Figure 4.1: marimba-mapping overview.

marimba-datamodel - We introduce the Nested Relational Model (NRM) and show how MARC 21 catalogues can be fully represented with this model. This data model provides a formal basis for extending the W3C R2RML language to deal with nested data. We call this model marimba-datamodel and introduce it in Section 4.2.

Schema extraction - We propose a method to extract a schema $\mathcal{S}$ in the marimbadatamodel out of a given library catalogue $\mathcal{C}$. In this way, we can model the problem of mapping library data sources into RDF as a "Global as View"1 data integration problem using this schema. This method is intended to overcome the lack of formal schema present in MARC 21 data sources and provide a mechanism to enable the domains experts to map existing catalogues into RDF. We describe this activity and its main features in Section 4.3.

marimba-sql - First, we introduce a recursive algebra for operating with nested relations in the marimba-datamodel, which will be used to provide a formal ground for our light-weight query language. We propose a query language, marimba-sql, that is translatable into the algebra for nested relations and is

\footnotetext{
${ }^{1}$ The "Global as View" (GAV) data integration approach has been described in Section 2.3.
} 
able to query nested relational data in the marimba-datamodel. We define the main features of the query language in Section 4.4. The goal of the marimba-sql query language is to provide a language to query nested data within our extension of the W3C R2RML mapping language.

marimba-rml language and processor - We extend the W3C R2RML mapping language with a formal framework to handle nested relational data in the marimbadatamodel. We present the key features of the marimba-rml language and its marimba-rml processor in Section 4.5.

Mapping template generation - The goal of this method is to bootstrap the mapping process by creating a set of mapping templates that will be used by library experts to define the mapping rules of a mapping $\mathcal{M}$ in terms of the extracted schema $\mathcal{S}$. We describe this method as well as its main features in Section 4.6.

\section{2 marimba-datamodel}

In this section, we introduce the Nested Relational Model and analyze the representation of MARC 21 data in this model.

\subsubsection{Definitions for the Nested Relational Model}

In this section, we present the Nested Relational Model proposed by Makinouchi [1977], using the definitions provided by Colby [1989].

Let $\mathcal{A}$ be the universal set of attribute names and relation scheme names. Let $\mathcal{D}$ be the domain of all the atomic attributes in $\mathcal{A}$.

Definition 4.2.1 (Relation scheme) A relation scheme of a relation is of the form $R(S)$; where $R \in \mathcal{A}$ is the relation scheme name, and $S$ is a list of the form $\left(A_{1}, A_{2}, \ldots, A_{n}\right)$.

Then, each $A_{i}$ is either an atomic attribute or a relation scheme of a sub-relation of $R(S)$. We will refer to a relation scheme using simply $R$ instead of $R(S)$. Further, an instance $r$ of a relation scheme $R$ is a set of ordered n-tuples of the form $\left(a_{1}, a_{2}, \ldots, a_{n}\right)$ such that:

Definition 4.2.2 (Atomic attribute) If $A_{i}$ is an atomic attribute, then $a_{i} \in \mathcal{D}$.

Definition 4.2.3 (Relation-valued attribute) If $A_{i}$ is a relation sheme of the form $R_{i}\left(S_{i}\right)$ then $R_{i}$ is called a relation-valued attribute of $R$ or sub-relation of $R$. 
Definition 4.2.4 (Relation) If $A_{i}$ is a relation scheme, then $a_{i}$ is an instance of $A_{i}$. An instance of a relation scheme is also referred to as a relation.

Definition 4.2.5 (Attribute names) Attr $(R)$ is the set of all (atomic and relation-valued) attribute names in $S$.

Definition 4.2.6 (Relation-valued attribute names) RAttr $(R)$ is the set of all relationvalued attribute names in $S$.

Definition 4.2.7 (Atomic attribute names) $F A t t r(R)$ is the set of all atomic attribute names in $S$.

Let $r$ be an instance of $R$ and let $t \in r$ (a tuple in relation $r$ ). If $A \in \operatorname{Attr}(R)$ then $t[A]$ is the value of $t$ in the column corresponding to $A$. If $B \subseteq \operatorname{Attr}(R)$ then $t[B]=t\left[A_{1}\right] t\left[A_{2}\right] \ldots t\left[A_{m}\right]$ where $A_{i} \in B(1 \leq i \leq m)$.

Further, let $c$ be condition on $R$ if

1. $c=\varnothing$

2. $c=a \theta b$ where

a) $a$ is an atomic attribute of $R$ and $b$ is an atomic attribute or an atomic value, $a$ and $b$ have compatible domains and $\theta \in\{\langle\rangle,, \leq, \geq,=, \neq\}$.

b) $a$ is a relation-valued attribute of $R$ and $b$ is relation-valued attribute of $R$ or an instance of the domain of $a$, and $\theta \in\{\subset, \subseteq,=, \neq, \supset, \supseteq\}$.

c) $b$ is a relation-valued attribute of $R$ and $a$ is a tuple in some instance of $b$ and $\theta \in\{\in, \notin\}$.

3. $c 1$ and $c 2$ are two conditions on $R$ and $c=c 1 \wedge c 2$ or $c=c 1 \vee c 2$ or $c=\neg c 1$

If $t$ is a tuple in some $r \in R$, then

1. If $c=\varnothing$ then $c(t)=$ true

2. If $c=a \theta b$ then either:

a) $c(t)=t[a] \theta t[b]$ if $a$ and $b$ are both attributes.

b) $c(t)=a \theta t[b]$ if only $b$ is an attribute.

c) $c(t)=t[a] \theta b$ if only $a$ is an attribute. 
3. $c(t)=c 1(t) \wedge c 2(t), c(t)=c 1(t) \vee c 2(t)$, and $c(t)=c 1(t)$ when $c=$ $c 1 \wedge c 2, c=c 1 \vee c 2$, and $c=c 1$ respectively.

\subsubsection{Representing MARC 21 records in the marimba-datamodel}

The goal of this section is to study the representation of MARC 21 records using the Nested Relational Model introduced in the previous section. In order to structure the study, we analyze each of the elements of the MARC 21 standard, and map them using the definitions of the NRM defined above. The elements and structure of the MARC 21 standard have been introduced in Section 2.1.1. As mentioned in Section 2.5, a MARC 21 catalogue $\mathcal{C}$ is a finite set of records. A record $r=(l, F)$ is defined by a leader $l$ and a non-empty set of fields $F=\left(f_{1}, \ldots, f_{n}\right)$. Further, $F \in(C F \cup D F)$, where $C F$ are control fields and DF are data fields. As shown in Figure 4.2, we can intuitively draw a nested relational table where each record is a row in a nested relation with the field 001 as primary key, which, as explained in Section 2.1.1, corresponds to the unique identifier of MARC 21 records.

Onwards, we will denote:

- Control and data fields using their three-digit code preceded by an $f$ symbol.

- Indicators using a digit preceded by an $i$ symbol.

- The positions within fixed-length fields using the position digit preceded by a $p$ symbol.

- Subfields using their alphabetic code (e.g., $t$ for the title subfield).

Using the definitions of Section 4.2.1, the complete table (or relation) shown in Figure 4.2 corresponds to $R(S)$ with $S=(f 001, f 005, f 100, f 700)$, where:

(i) $f 001$ and $f 005$ are atomic attributes, thus $F A t t r(S)=(f 001, f 005)$;

(ii) $f 100$ and $f 700$, are relation-valued attributes, thus $R \operatorname{Attr}(S)=(f 100, f 700)$. We name these relation-valued attributes $R_{f 100}\left(S_{f 100}\right)$ and $R_{f 700}\left(S_{f 700}\right)$ respectively, where $S_{f 100}=(i 0, i 1, a, d)$ and $S_{f 700}=(i 0, i 1, a, d)$. Finally, each of the attributes of $S_{f 100}$ and $S_{f 700}$ are atomic attributes.

We discuss each of the variants for the different types of field below. Please note that the examples correspond to a sub-relation scheme of the complete relation scheme bib shown in Figure 4.2 except from the leader which is not shown in the figure for simplicity: 


\begin{tabular}{|c|c|c|c|c|c|c|c|c|c|}
\hline \multirow[t]{2}{*}{001} & \multirow[t]{2}{*}{005} & \multicolumn{4}{|c|}{100} & \multicolumn{4}{|c|}{700} \\
\hline & & 0 & 1 & $\mathrm{a}$ & d & 0 & 1 & $\mathrm{a}$ & d \\
\hline \multirow[t]{2}{*}{ bima0000007176 } & \multirow[t]{2}{*}{20150511} & \multirow[t]{2}{*}{1} & \multirow[t]{2}{*}{0} & \multirow[t]{2}{*}{$\begin{array}{l}\text { Cervantes } \\
\text { Saavedra, } \\
\text { Miguel de }\end{array}$} & \multirow[t]{2}{*}{$1547-1616$} & 1 & null & Ferrer, Josep & fl. s. XVII \\
\hline & & & & & & 1 & null & $\begin{array}{l}\text { Mey, Pedro } \\
\text { Patricio }\end{array}$ & fl. $1582-1623$ \\
\hline bimo 0000776072 & 20091223 & 1 & 0 & $\begin{array}{l}\text { Diago, } \\
\text { Francisco }\end{array}$ & null & & & null & \\
\hline
\end{tabular}

Figure 4.2: Table representation of two MARC 21 bibliographic records in the Nested Relational Model (we denote this table as the relation $b i b$ ).

Leader - The leader field has a fixed length of 24 characters where each position has an specific meaning. Therefore, in the NRM this field corresponds to a relationvalued attribute where each of the positions corresponds to an atomic attribute. Figure 4.3 shows the relation scheme $R_{\text {leader }}\left(S_{\text {leader }}\right)$ that is composed by a set of 24 atomic attributes $S_{\text {leader }}=(p 00, p 01, p 02, \ldots, p 23)$ corresponding to each of the character positions.

\begin{tabular}{|c|c|c|c|c|c|c|c|}
\hline \multicolumn{8}{|l|}{ leader } \\
\hline p00 & p01 & $\mathrm{p} 02$ & . . & p06 & p07 & . & p23 \\
\hline$b$ & $\mathrm{a}$ & C & & $b$ & C & & $\mathrm{a}$ \\
\hline
\end{tabular}

Figure 4.3: Example of the leader field and its representation as relation-valued attributes

Control Fields - These fields can be further classified into two types: $i$ ) fixed-length positional fields; and, ii) single-valued fields. Fixed-length positional fields correspond in the NRM to relation-valued attributes where each of the positions corresponds to an atomic attribute. Single-valued fields correspond to atomic 
attributes. Figure 4.4 depicts an example of two single-valued control fields (f001,f005) and Figure 4.5 depicts an example of a fixed-length positional field.

\begin{tabular}{|l|l|}
\hline \multicolumn{2}{|l|}{ f001 } \\
\multicolumn{2}{|l|}{ b005 } \\
\hline bima0000007176 & 20150511 \\
\hline bimo0000776072 & 20091223 \\
\hline
\end{tabular}

Figure 4.4: Example of two single-value control fields corresponding to the attomic attributes f001 and f005

\begin{tabular}{|ccccccccc|}
\hline 008 & & & & & & & \\
\hline p00 & p01 & p02 & p35 & p36 & p37 & $\ldots$ & p39 \\
$\#$ & $\#$ & $\#$ & s & p & a & \\
\hline
\end{tabular}

Figure 4.5: Example of a fixed-length control field corresponding to the relation-value attribute f008. The symbol \# is used for undefined values in the MARC 21 standard. Positions 35-37 indicate the language of the resource, Spanish in the example.

Data Fields - These fields are further specified by subfields and two indicator characters. Therefore, data fields correspond to relation-valued attributes, where indicators and subfield codes correspond to the relation schemes of the subrelations within the attribute.

Furthermore, some Data Fields are repeatable (e.g., field $f 700^{2}$ ), which is represented as several distinct tuples in the relation of the field. Figure 4.6 shows a single tuple of a relation from a non-repeatable data field. Figure 4.7 shows two tuples of a relation from a repeatable data field corresponding to the editor (i.e., Josep Ferrer) and the printer (i.e., Mey, Pedro Patricio) of the edition of "Don Quixote".

\footnotetext{
${ }^{2}$ The field 700 in the MARC 21 format for Bibliographic records is used to describe persons having some form of responsibility for the creation of the work (authorship, publishing, etc.)
} 


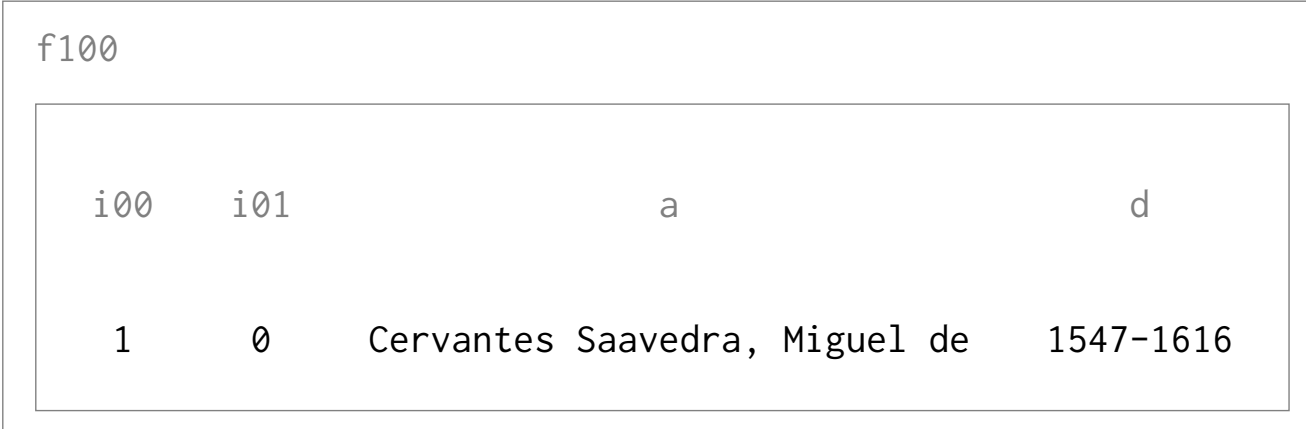

Figure 4.6: Example of a non-repeatable data field and its representation a relationvalued attribute

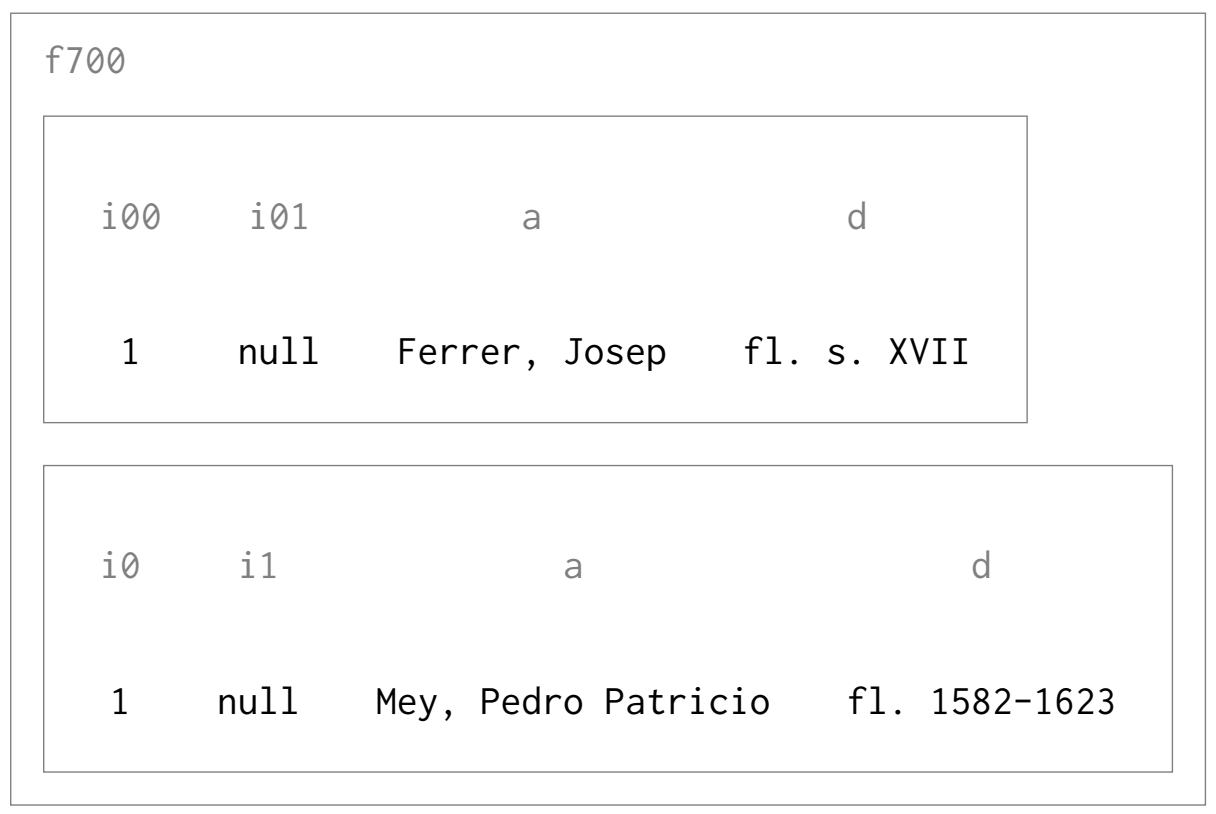

Figure 4.7: Example of two tuples of a repeatable data field and its representation as relation-valued attribute

Given the definitions and analysis above, we have shown analytically that MARC 21 records can be fully represented in the NRM. We call this model marimba-datamodel. To conclude this analysis, in Table 4.1, we summarize the complete mapping from the MARC 21 standard elements to the marimba-datamodel. The analysis and complete mapping presented in this section, sustain the validation of our first hypothesis, which we formulated as follows The structure of MARC 21 records can be fully represented using an abstract relational model.. To complete this analysis, we present in the next section a novel extraction algorithm to extract the schema of MARC 21 data sources represented in the marimba-datamodel. 


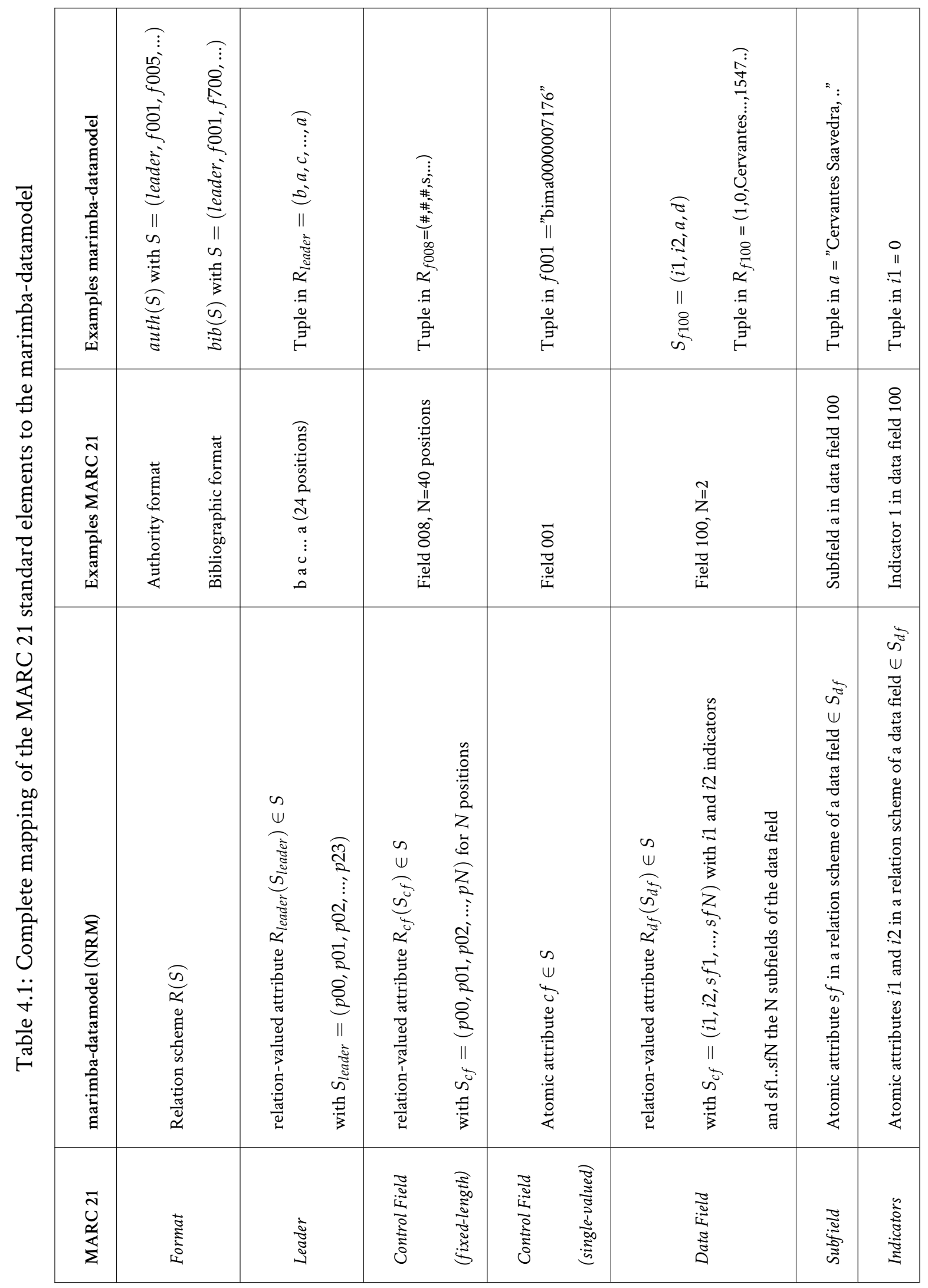




\subsection{Schema extraction}

As discussed in Chapter 2, one of the challenges of transforming library data into a semantic representation is to extract the schema of MARC 21 data sources. We call this activity schema extraction.

The term "schema extraction" has been extensively used in the field of database research (Batini et al. [1986a], Andersson [1995]), XML (Hegewald et al. [2006]), and more recently in the context of tabular data on the Web (Adelfio and Samet [2013]). Schema extraction can be loosely defined as the activity of extracting the implicit logical structure of one or more data sources. For instance, given that XML documents contain data as well as their implicit logical structure, the task of schema extraction from XML documents consists in automatically specifying their XML Schema directly from the data. ${ }^{3}$ In the context of this thesis, we define schema extraction as a method for extracting the logical structure of a given set of library records that conform to the MARC 21 standard. In this section, we introduce a novel schema extraction method for MARC 21 data sources. First, we discuss the information that can be extracted from MARC 21 data sources. Second, we introduce our schema representation method. Finally, we define the schema extraction method.

\subsubsection{Information in MARC 21 records}

The following information is available for each MARC 21 record:

Record level information - Information at the level of record can be extracted in the form of statistics. The most relevant statistics to register are: i) distribution of fields (i.e., in how many records a field appears?), and ii) repetition of fields (i.e., what fields appear more than once in any of the records?). This information may be potentially useful for library experts to define the mapping rules, as well as to identify potential cataloging errors.

Leader - As introduced in Chapter 2, the leader field ${ }^{4}$ is a field that carries information for processing the records (e.g., record length), and potentially useful information for mapping, such as the type of record (e.g., musical sound recording, cartographic material). The field has a fixed length of 24 characters, where

\footnotetext{
${ }^{3}$ The W3C XML Schema specification provides the ability of defining a machine-processable schema for XML documents: http: //www.w3 .org/XML/Schema

${ }^{4}$ https://www. loc.gov/marc/bibliographic/bdleader. html (Last viewed 19/01/2016)
} 
different positions have specific meanings. For instance, the character at position 06 defines the type of record property. Therefore, a method for extracting the schema should be able to extract information from those positions that are relevant for mapping.

Control fields - Control fields provide a great deal of information for mapping. Control fields have no indicator or subfield codes, thus the schema extraction should work with tags (e.g., 001) and values (e.g., XX174567). Moreover, similarly to the leader field, some control fields are fixed-length fields with different positions indicating different properties about the resource being described in the record. For instance, the field 008 provides relevant semantic information of the resource (e.g., characters at positions 35-37 indicate the language of the resource).

Data fields - Data fields contain major information for mapping, such as author name, title of work, etc. These fields have tag, indicators, and subfield codes that should be taken into account by the schema extraction approach. It is worth noting that the presence of indicators may modify the meaning of certain subfields, thus the schema extraction approach must take them into account. Moreover, fields and subfields can be repeatable (i.e., a record can have several instances of the same field or subfield).

In summary, a schema extraction approach can gather information at different levels for each record component. More specifically, the information available is the following:

- Statistics for record-level information.

- Tags for control fields, data fields, indicators, and subfield codes.

- The nature of data fields and subfields (repeatable or single-valued).

- Values of fixed-length positions of the leader and the fixed-length control fields.

In the following section and based on this analysis, we propose our novel algorith for extracting the schema of MARC 21 data sources. 


\subsubsection{Method}

In this section, we describe our method for extracting the schema of library catalogues. The input of the schema extraction method is a catalogue $\mathcal{C}$ and the outputs are: $i$ ) a schema $\mathcal{S}$ in the marimba-datamodel, and $i$ ) a set of record-level statistics $\mathcal{R}$. We compute the schema and the statistics by means of a novel algorithm, whose pseudocode is presented in Algorithm 1 and which consists of six steps:

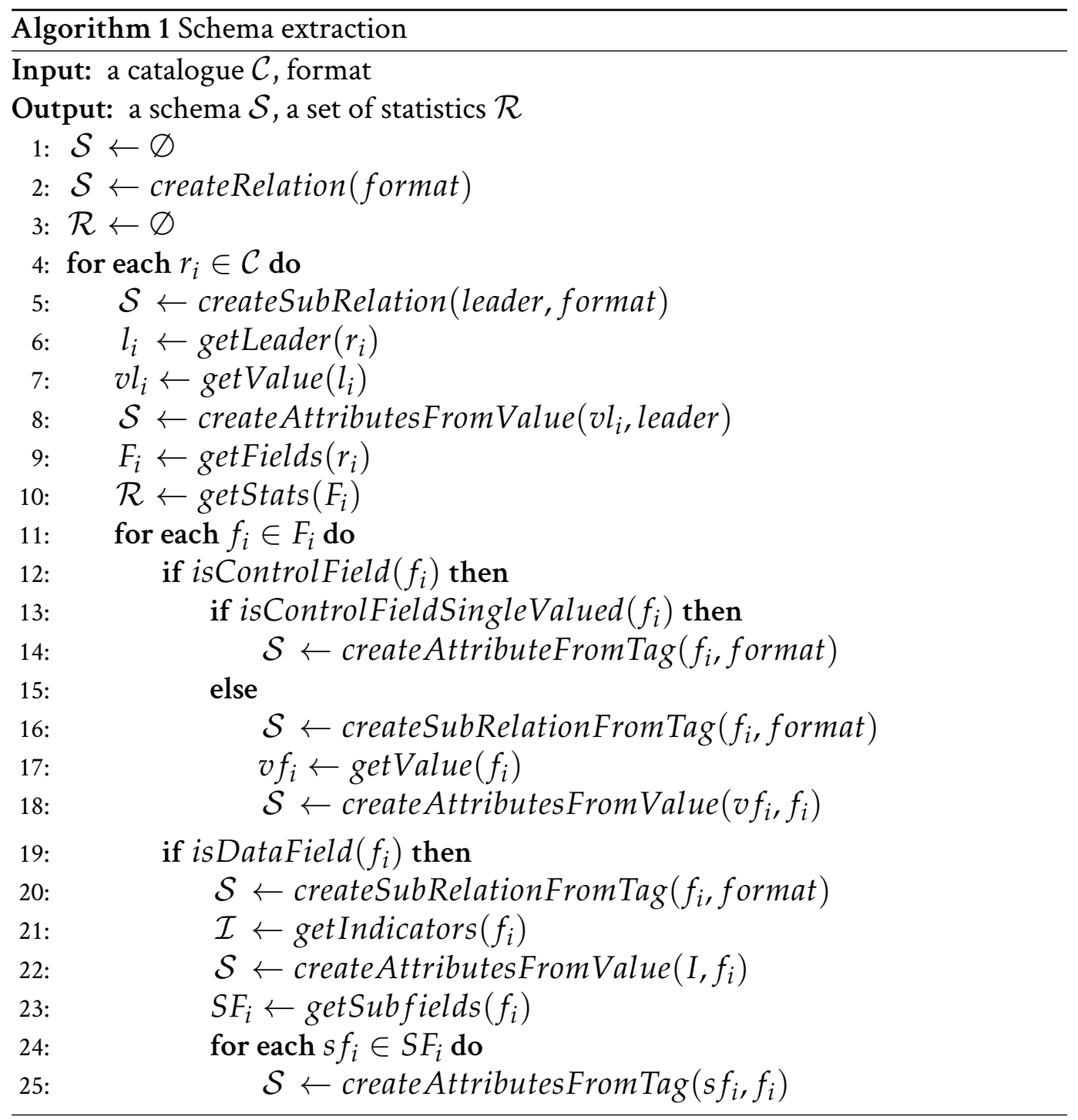

1. Initialization (lines 1-3). We start by initializing the schema $\mathcal{S}$ with a relation for the format (i.e., authority or bibliographic) and the set of statistics $\mathcal{R}$. Then, the method iterates over the set of records of the catalogue $\mathcal{C}$ that will be processed by the next steps. It is worth noting that the next steps (i.e., 2, 3, 4, 5) are applied to each of the records. 
2. Leader extraction (lines 5-8). As discussed in Section 4.3.1, the leader is a fixedlength field that contains potentially useful information in certain positions of its value. Thus, in this step we first create a sub-relation, named leader (line 6), of the relation format (from step 1), and then extract the value of those positions and create an atomicattribute for each of them (lines 7 and 8 ).

3. Statistics gathering (line 10). In order to gather statistics about the records in the catalogue, in this step we extract the following features for each field: $i$ ) the repetition of the field within the record, this feature will indicate which fields are repeatable; and ii) the number of occurences of the field within the records, this feature will indicate the overall frequency of fields within the catalogue.

Then, we extract the fields $F_{i}$ for the record $r_{i}$ (line 9) and perform the following steps:

4. Control fields extraction (lines 12-18). In this step we are interested in extracting information from those control fields that are: $i$ ) single-valued (lines 13-14), and ii) fixed-length and contain descriptive positions, namely the fields 006, 007, and 008 (lines 13-14). For single-valued fields, we create an atomic-attribute of the relation format using the tag of each of these fields. For fixed-length fields, we create a sub-relation of the relation format, and then we create an atomic-attribute of that subrelation for each of the positions.

5. Data fields extraction (lines 19-25). In this step we are interested in the complete set of fields, their indicators and subfields. In this case, we are not interested in the values, as there are no fixed-length data fields. Thus, we first create a sub-relation of the format relation for each field. Then, we extract the indicators and create atomicattributes of the sub-relation created, loop through the set of subfields, and create a atomic-attribute with the subfield tag.

After the execution of the schema extraction method, we have the schema $\mathcal{S}$ in the marimba-datamodel, and the set statistics $\mathcal{R}$. It is worth noting that during the schema extraction algorithm, we register the nature of the data fields and subfields (i.e., repeatable or single-valued). The schema and the statistics are used by the mapping template generation method that we present in Section 4.6. Finally, we show an example of an extracted schema in the following listing. Please note that we use the keyword TABLE to indicate relations, ITEM TABLE to indicate sub-relations, ITEM to indicate atomic-attributes, and REPEATABLE to indicate that a field or subfield is repeatable (i.e., can define several values). 
Listing 4.1: Sample of a MARC 21 schema extracted with our algorithm

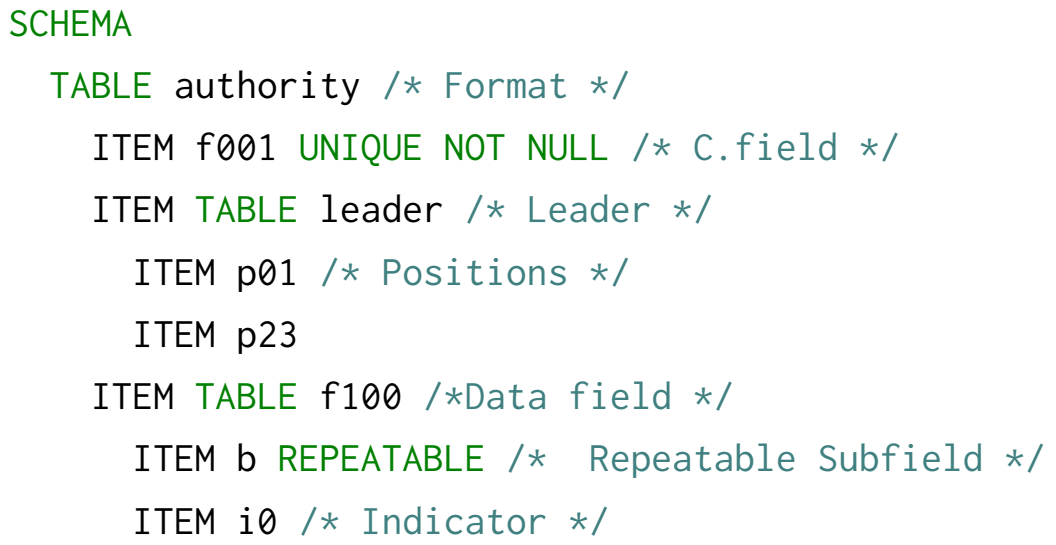

\section{4 marimba-sql query language}

In this section, we describe marimba-sql, the query language to be used to query nested data in the marimba-rml mapping language, our extended R2RML mapping language. marimba-sql is a subset of the SQL/NF language that was proposed by Roth et al. [1987] to deal with relational databases that are not in first-normal-form and in particular the nested relational model. First, we present the recursive algebra of Colby [1989] that provides a powerful abstraction to deal with the Nested Relational Model and that will be used to define our proposed query language marimba-sql. Second, we introduce the main features of the SQL/NF language. Finally, we present marimbasql as a subset of SQL/NF and its translation to the recursive algebra.

\subsubsection{Recursive algebra for the marimba-datamodel}

The goal of this section provide a concise formalism to handle catalogue data in NRM. This mechanism is inspired by the research in query languages for the NRM developed over the years. On the one hand, non-recursive algebras have been proposed (e.g., Thomas and Fischer [1986]). One of the issues with these algebras is that they operate at the outermost level of relations. Therefore, they suffer from a lack of conciseness and require series of complicated nest, unnest, and index operations to deal with sub-relations. On the other hand, recursive algebras like the one proposed by Colby [1989], provide a more concise and elegant way of operating with nested relational data. The rationale for using this recursive algebra is that it provides a good basis for optimization of the transformation process, which is out of the scope of this thesis, as 
well as for the application of modern approaches for large-scale data querying and processing. A notable example of the use of this algebra is the work on Dremel by Melnik et al. [2010], which provides read-only in-place querying of very large datasets. The goal of this section is to introduce the core operators of this algebra that will serve as the basic formalism to support our query language marimba-sql.

The operators of this algebra are recursively defined, what means that they can apply themselves to the sub-relations at different levels of a relation. The algebra defines four unary operators (selection, projection, nest, and unnest) and five binary operators (union, difference, intersection, cartesian product, and equi-join). marimba-sql will be designed to provide a minimal query language. Thus, we will not introduce the nest and unnest unary operators. The nest and unnest operators provide data restructuring mechanisms that are out of the scope of the constructs proposed in this thesis. We introduce below the operators that will be used in this thesis.

\section{Selection $(\sigma)$}

The selection operator retrieves all the tuples in the relation which satisfy a certain condition. When the selection condition involves attributes that are not at the outermost level (i.e., attributes inside a relation-valued attribute), a non-recursive algebra requires a series of operations to flatten the involved sub-relations. The recursive version can operate on attributes at any level without having to flatten the relations.

Let $R L=\left(R_{1 c 1} L_{1}, R_{2 c 2} L_{2}, \ldots, R_{n c n} L_{n}\right)$ be a "select list", where each $R_{i}$ is a relation-valued attribute of $R, c_{i}$ is a condition on $R_{i}$, and $L_{i}$ is a (possibly empty) select list of $R_{i}$. The selection can then be defined in terms of $L$ :

$$
\sigma\left(r_{c}\left(R_{1 c 1} L_{1}, R_{2 c 2} L_{2}, \ldots, R_{n c n} L_{n}\right)\right)
$$

where $r$ is a relation with relation scheme $R$ and $c$ is a condition on $R$.

Example 4.4.1 (Selection of subfield within a data field) A selection operation over the relation bib shown in Figure 4.2 is $\sigma\left(\right.$ bib $\left.\left(f 100_{d=1547-1616}\right)\right)$, that selects the first row of the table.

Example 4.4.2 (Selection of indicator within a data field) $\sigma\left(b i b\left(f 100_{i 1=0}\right)\right)$ selects both rows as they have the same value for indicator 1 . 


\section{Projection (П)}

The projection operator "projects" out the columns corresponding to the attributes in the set $A$. The recursive definition of projection defines a list $L$ in the same way as for the selection operator.

Let $L=\left(R_{1} L_{1}, R_{1} L_{2}, \ldots, R_{n} L_{n}\right)$ be a "project list" of $R$, where each $R_{i}$ is an attribute of $R$ (relation-valued or atomic), and $L_{i}$ is a project list of $R_{i}$ that is empty if $R_{i}$ is an atomic-attribute. Then, a projection can be defined as:

$$
\pi\left(\left(R_{1} L_{1}, \ldots, R_{i} L_{i}\right) r\right)
$$

where $r$ is a relation with relation scheme $R$.

Example 4.4.3 (Projection of a control field and a subfield.) A possible projection operation over the table $r_{1}$ shown in Figure 4.2 is $\pi((f 001, f 100(a))$ bib), that projects the relation shown in Figure 4.8

Example 4.4.4 (Projection of a data field and two subfields.) The projection operation $\pi((f 001, f 100(a, d)) b i b))$ over the relation bib adds the attribute $d$ containing the dates to the relation shown in Figure 4.8.

Example 4.4.5 (Combination of selection and projection.) The combination of selection and projection $\Pi\left((f 001, f 100(a, d))\left(\sigma\left(b i b\left(100_{a=\text { Diago,Francisco }}\right)\right)\right)\right)$ over the relation bib selects and projects the second row of the relation shown in Figure 4.8.

\begin{tabular}{|c|c|}
\hline 001 & 100 \\
\cline { 2 - 2 } & a \\
\hline bima0000007176 & $\begin{array}{c}\text { Cervantes } \\
\text { Saavedra, } \\
\text { Miguel de }\end{array}$ \\
\hline bimo0000776072 & $\begin{array}{c}\text { Diago, } \\
\text { Francisco }\end{array}$ \\
\hline
\end{tabular}

Figure 4.8: Relation resulting from applying a projection operation to $b i b$ of Figure 4.2 


\section{Union ( $\cup$ ), Intersection $(\cap)$, Difference $(-)$}

The non-recursive version of these operators operate at the level of the outermost relation considering entire tuples. Let $r_{1}, r_{2} \in R$, then

$$
\begin{aligned}
& r_{1} \bigcup r_{2}=\left\{t \mid t \in r_{1} \vee t \in r_{2}\right\} \\
& r_{1}-r_{2}=\left\{t \mid t \in r_{1} \wedge t \notin r_{2}\right\} \\
& r_{1} \bigcap r_{2}=\left\{t \mid t \in r_{1} \wedge t \in r_{2}\right\}
\end{aligned}
$$

In this way, if we perform a union of the tuples (Cervantes,(Quijote,Novelas Ejemplares)) and (Cervantes, (Galatea)) the result will be a relation that has the tuples (Cervantes,(Quijote,Novelas Ejemplares)) and (Cervantes,(Galatea)). However, we might want to receive the tuple (Cervantes,(Quijote,Novelas Ejemplares,Galatea)) instead. This means operating at the sub-relation level. The recursive version of the union, intersection, and difference, provides the ability to work at this level by recursively defining $\bigcup^{e}\left(r_{1}, r_{2}\right), \bigcap^{e}\left(r_{1}, r_{2}\right)$ and $-^{e}\left(r_{1}, r_{2}\right)$.

\section{Cartesian-Product $(\times)$,Equi-join $\left(\star_{=}\right)$}

The recursive nested relational algebra also provides two operators to join the tuples of a relation to tuples inside nested relation-valued attributes. The recursive version provides two new operators $\times$ and $\aleph_{=}$. These operators allow joins and cross-products to be applied to a relation or a relation-valued attribute and another relation. Thus, these operations are not symmetric, meaning that one operand can be a relation or a sub-relation while the other operand must be a relation. We introduce the two operators below.

We start by defining the Cartesian-Product. Let $L$ be an "access path" of $R$. Then, $\mathrm{L}$ is either: $(i)$ an empty list, or $(i i) R_{i}\left(L_{i}\right)$ where $R_{i}$ is a relation-valued attribute of $R$ and $L_{i}$ is an access path of $R_{i}$.

Let $r$ and $q$ be two relations with relation schemes $R$ and $Q$ respectively. The Cartesian-Product of $r$ and $q$ is either: $i)(r, q)$ when $r$ and $q$ are relations, or $i i)$ $\left(r\left(R_{i}\left(L_{i}\right)\right), q\right)$ where $R_{i}\left(L_{i}\right)$ is an access path of $R_{i}$. Notice that common attributes in $R$ and $Q$ are renamed in order to resolve ambiguity.

Based on the above definition, we can define the Equi-Join operator as follows. Let $R$ and $Q$ be two relation schemes. Let $L$ be 'equi-join' list of $R$ and $Q$. Then $L$ is either: $(i)$ a list of the form $\left(R_{1}, \ldots, R_{n}\right)$ where each $R_{i} \in \operatorname{Attr}(R) \operatorname{Attr}(Q)$, or 
(ii) a list of the form $R_{i}\left(L_{i}\right)$ where $R_{i}$ is a relation-valued attribute of $R$ and $L_{i}$ is an equi-join list of $R_{i}$ and $Q$.

Let $r$ and $q$ be two relations with relation schemes $R$ and $Q$ respectively. The Equi-Join of $r$ and $q$ is either: $i) \aleph_{=}\left(r\left(R_{1}, \ldots, R_{n}\right), q\right)$, or $\left.i i\right) \aleph_{=}\left(r\left(R_{i}\left(L_{i}\right)\right), q\right)$ where $R_{i}\left(L_{i}\right)$ is an access path of $R_{i}$.

\subsubsection{SQL/NF}

In this section, we briefly introduce the SQL/NF language, which is a superset of our proposed query language. SQL/NF is an extension of standard SQL version of 1993 (Date and Darwen [1993]) to operate on nested relational data. SQL/NF adds to SQL the ability to define nested relations in the data definition language and query these relations directly in the extended data manipulation language. Furthermore, changes are made to the standard SQL to allow the data and relation restructuring operations NEST and UNNEST. It is worth mentioning that although there are other proposals in the literature for query languages over NRM (Korth and Roth [1989]), SQL/NF is the less complex and the closest to the standard SQL language. The core idea under SQL/NF is that wherever a constant or a scalar-value may appear in SQL, a relation or expression evaluating to a relation may appear in SQL/NF too.

SQL/NF operates with schemes and relations that comply with the definitions of the NRM given in Section 4.2.1. The only restriction is that relations must be in partitioned normal form (PNF). A relation is in PNF if the atomic attributes of each nested relation form a key for that relation. We must therefore keep this property in mind when representing MARC 21 catalogue data in the NRM. The SQL/NF queries conform to following structure:

Listing 4.2: SQL query structure

\section{SELECT attribute-list \\ FROM relation-list \\ WHERE predicate}

The standard SQL language has the closure property where the result of a SelectFrom-Where (SFW) query is itself a relation. Following the principle of orthogonality, a query language should allow SFW expressions wherever a relation name can exist in a query. In standard SQL that means allowing nested SFW expressions in the FROM clause. In SQL/NF since attributes can be relation-valued, SFW expressions are also allowed in the SELECT clause. Moreover, SFW expressions are allowed in the 
WHERE clause to enable predicates to operate with attributes within nested relations. Another feature of SQL/NF is its ability to select the complete set of attributes of a relation directly by using the relation name without the need of a SELECT FROM clause. For instance, the following two queries are equivalent:

Listing 4.3: SQL/NF simplified notation

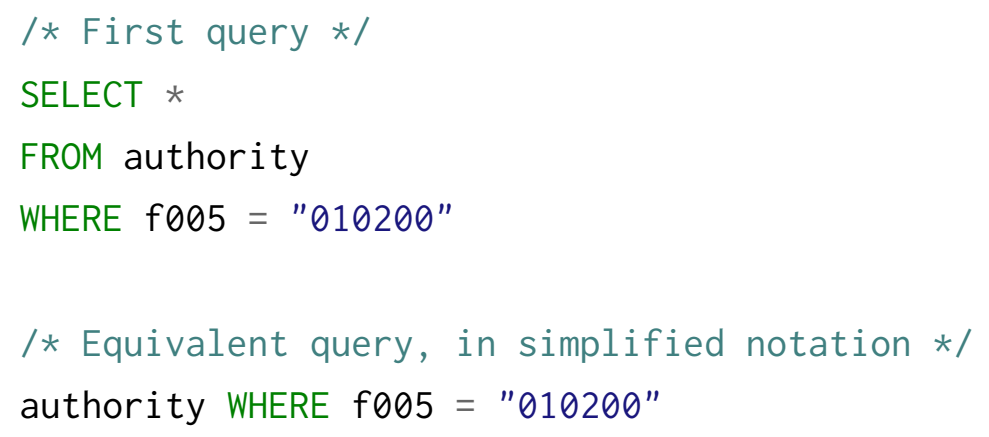

The combination of nested SFW expressions and the simplified notation for selecting relations results in compact and readable queries such as the one shown below, where we refer to the subfields $a$ and $t$ within the field 100:

Listing 4.4: Nested SQL/NF expression

authority WHERE EXISTS ( $f 100$ WHERE a $!=$ NULL AND $t=$ NULL)

Finally, another relevant feature of SQL/NF is the application of the principle of orthogonality to functions (e.g., SUM, AVG) by allowing every function to be applied to any expression that returns a relation. For instance, we can apply the function COUNT to a relation-valued attribute such as the field 700 to retrieve the number of instances of the field for each record in the authority relation:

Listing 4.5: SQL/NF function

SELECT f001, COUNT(f700)

FROM authority

In this section we have briefly introduced some of the most useful features of SQL/NF. The complete details and the BNF grammar are available in the original paper of Roth et al. [1987]. In the next section, we describe the subset of SQL/NF that correspond to our proposed query language: marimba-sql 


\subsection{3 marimba-sql syntax}

The W3C R2RML standard provides the means for using the standard SQL language to query relational data. In order to extend this mapping language to deal with nested relational data, we propose a new query language. Our main goal is to provide a minimal query language that can be used within the extended R2RML mappings defined in the marimba-rml mapping language, which will be presented in Section 4.5. Thus, we focus exclusively on the data query language constructs of SQL/NF. We enumerate the main characteristics of the marimba-sq $1^{5}$ query language with respect to SQL/NF below:

1. Data query construct: The language provides support for the SELECT construct.

2. Functions: The language provides support for the following functions: SUM and COUNT.

3. Operators: The language provides support for the following operators: UNION, DIFFERENCE, INTERSECT, and SUBSET OF.

4. Our language does not include support for the NEST and UNNEST data and relation restructuring constructs. The reasons for not including these operations are that: $i$ ) they will add complexity to our R2RML extension in terms of its operational semantics, and $i i$ ) they are very costly operations when dealing with millions of rows (i.e., records)

Listing 4.6 shows several examples of queries to select bibliographic records based on their type of resource, which is defined in different positions of the leader and of the field 007.

Listing 4.6: marimba-sql expression examples

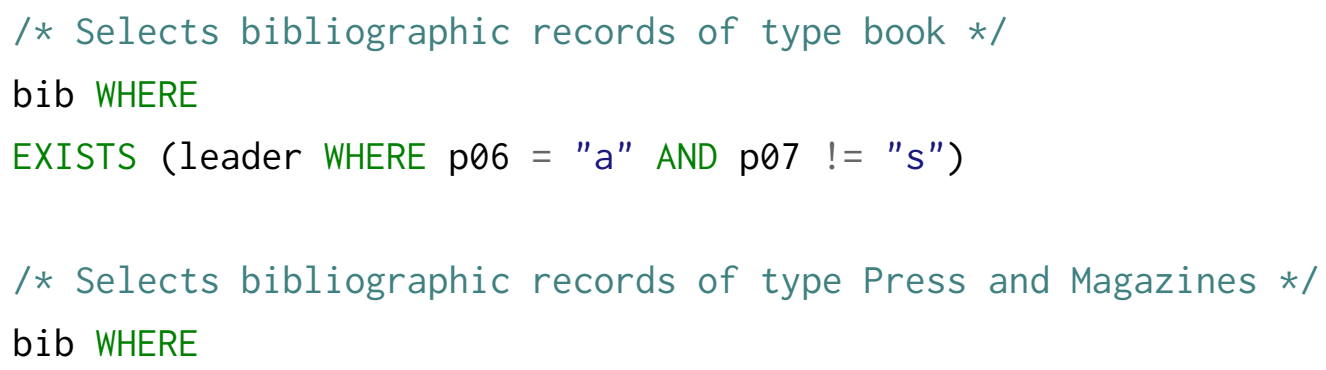

\footnotetext{
${ }^{5}$ We provide the complete BNF grammar definition of the marimba-sql language in Appendix A.
} 
EXISTS (leader WHERE p06 = "a" AND p07 = "s")

/* Selects bibliographic records of type Drawing */

bib WHERE

EXISTS (leader WHERE $p 6=" k "$ ) AND EXISTS ( f007 WHERE $p 2=" d "$ )

\subsection{4 marimba-sql to recursive algebra translation}

In this section, we briefly describe the translation rules from marimba-sql to the recursive algebra of Colby [1989], based on the translation method proposed by Schnepf [1990].

The three clauses of a query (SELECT,FROM, WHERE) shown in Listing 4.2 can be translated into the recursive algebra of Colby [1989] using the relational operators select $(\sigma)$, and project $(\pi)$ and the cartesian-product $(\times)$. Notice that the attribute and predicate list can contain nested queries that comply to the same SFW as described above. The result of the translation of a query into nested relational algebra is an expression of the form:

$$
\pi((P) \sigma(S))
$$

where $P=\left(R_{1} L_{1}, \ldots, R_{n} L_{n}\right)$ is a "projection list", and $S$ is a "select list" potentially including several cartesian products. To obtain these expressions, the translation procedure is performed according to a set of rules. The complete translation procedure is described in Schnepf [1990]. We present below two example translations from marimba-sql into the recursive algebra using the translation procedure.

If we apply the translation procedure, the query "SELECT fO01 (SELECT a FROM f100) FROM bib" is translated into:

$$
\pi((f 001, f 100(a)) \sigma(b i b))
$$

If we add some conditions, the query "SELECT f001 (SELECT a FROM f100 WHERE $a=" M i g u e l "$ ) FROM bib" is then translated into:

$$
\pi\left((f 001, f 100(a)) \sigma\left(b i b\left(f 100_{a=\text { Miguel }}\right)\right)\right.
$$

\section{5 marimba-rml language}

The goal of this section is to define the syntax and operational semantics of marimba$\mathbf{r m l}$, the mapping language proposed in this thesis. marimba-rml is based on R2RML 
and addresses the limitations of KR2RML for querying nested data. We extend R2RML to provide a more systematic method to deal with nested relations and mappings. We define marimba-rml by combining the W3C R2RML mapping language, marimbadatamodel, and the marimba-sql query language.

\subsection{1 marimba-rml syntax}

In this section we propose an extension of the R2RML mapping language to extend its capabilities to deal with the nested relational data defined by the model marimbadatamodel. This extension is guided by the following goals:

1. Provide a mechanism to create R2RML views by explictly querying data in a nested relational model. This mechanism overcomes a limitation of KR2RML, which also works with materialized views, but where views are handled and generated outside the mapping specification (i.e., they are generated by users within the Karma tool).

2. Provide a method to select columns within nested relations in a systematic and orthogonal way. This feature is included in KR2RML through the use of JSON arrays, and therefore with very limited support for sophisticated queries (e.g., joins or projections).

To pursue the aforementioned goals, we propose a re-interpretation of several constructs of the R2RML language that we describe below. Every other R2RML construct not mentioned in this section, remains as defined in the official W3C Recommendation.

\section{rr:LogicalTable}

In the R2RML specification a logical table, $r r$ :LogicalTable, is either: i) a SQL base table or view, or ii) an R2RML view. An R2RML view is a logical table whose contents are the result of executing a SQL query against the source database. It is defined by exactly one valid SQL expression indicated in the $r r$ : sqlQuery property. A SQL query is a SELECT query in the SQL language that can be executed over the input database to produce multiple rows with no duplicate columns names.

Furthermore, an R2RML view may have one or more SQL version identifiers through the property $r r$ :sqlVersion. A SQL version identifier must be a valid IRI. We notice that beyond the official SQL version (i.e., Core SQL 2008), other versions 
can be defined outside the recommendation. In fact, a list of alternative versions is available, maintained, and updated by the W3C. ${ }^{6}$ Therefore, in marimba-rml we specify the query language as marimba-sql by indicating it with a valid IRI. ${ }^{7}$

As our target source are MARC 21 records represented as NRM, we only allow R2RML views. It is worth noting that while in the R2RML mappings and processors, the rr:sqlQuery is assumed to produce multiple rows with atomic columns, in marimba-rml a marimba-sql query produces multiple relations that may contain both atomic and relation-valued attributes. We discuss the implications of this change in the next section.

Example 4.5.1 (R2RML view for persons using marimba-sql) Let us imagine that we want to select and transform personal information from an authority catalogue. One way of identifying persons is to analyze the presence or absence of certain subfields within the main access point (i.e., field 100). Therefore, we can define a nested query to define an R2RML view corresponding to records describing persons ${ }^{8}$ in the authority catalogue shown in the following listing. In the example, we select every authority record without a title subfield (\$t).

Listing 4.7: marimba-rml logical table for person

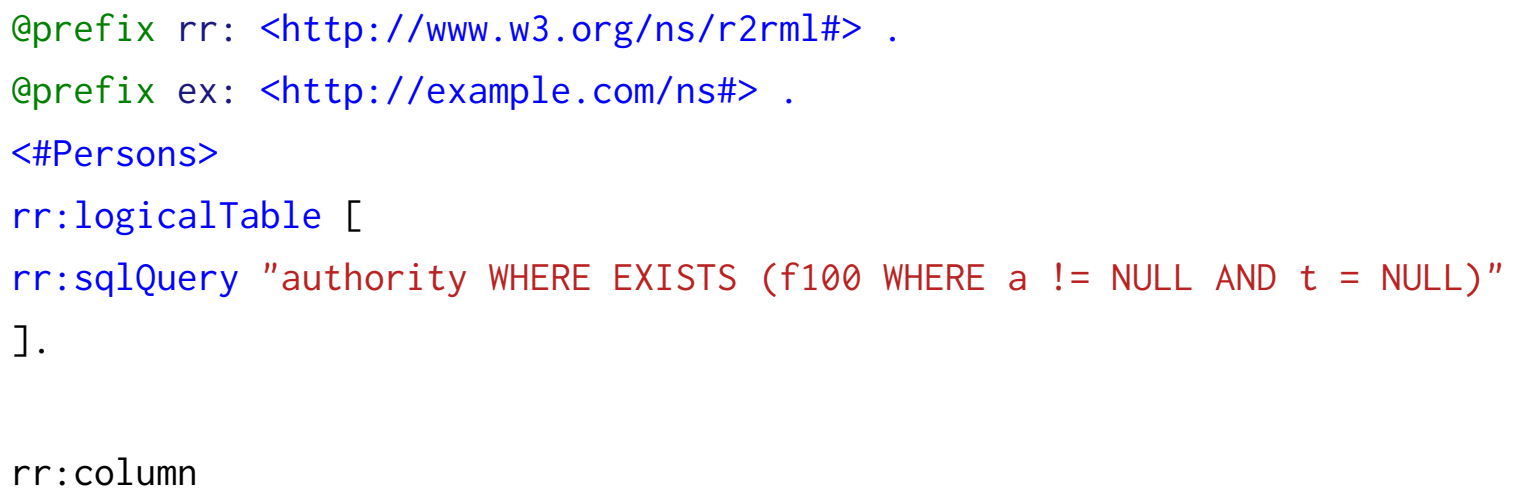

In the R2RML specification, a column ( $r$ : column) defines a column-valued term map. The value of $r$ : column must be a valid column name.

In marimba-rml, we process relations potentially containing relation-valued attributes. To keep our approach aligned with the specification of the nested relational model, we allow the application of SELECT queries to indicate either an atomic attribute, or navigate to atomic attributes within a nested relation. Specifically, the

\footnotetext{
${ }^{6}$ https://www.w3.org/2001/sw/wiki/RDB2RDF/SQL_Version_IRIs

${ }^{7}$ http://marimba4lib.com/ns/r2rml/marimba-sql. Please note that this URI is not dereferenceable.

${ }^{8}$ In the example we indicate that a person is described in a record that contains a personal name (i.e., subfield a), but does not contain a title (i.e., subfield t).
} 
value of a $r$ : column must be a valid SELECT marimba-sql query over the relations produced in the R2RML view. This query can be nested and must produce zero or more values of exactly one atomic attribute.

Example 4.5.2 (marimba-rml column properties to describe persons) We now want to map some authority fields and subfields to datatype properties. We can define two queries to select an atomic attribute (e.g., field 001) and an atomic attribute within a nested relation (e.g., subfield a offield 100 ) as follows

\section{Listing 4.8: marimba-rml columns for properties of person}

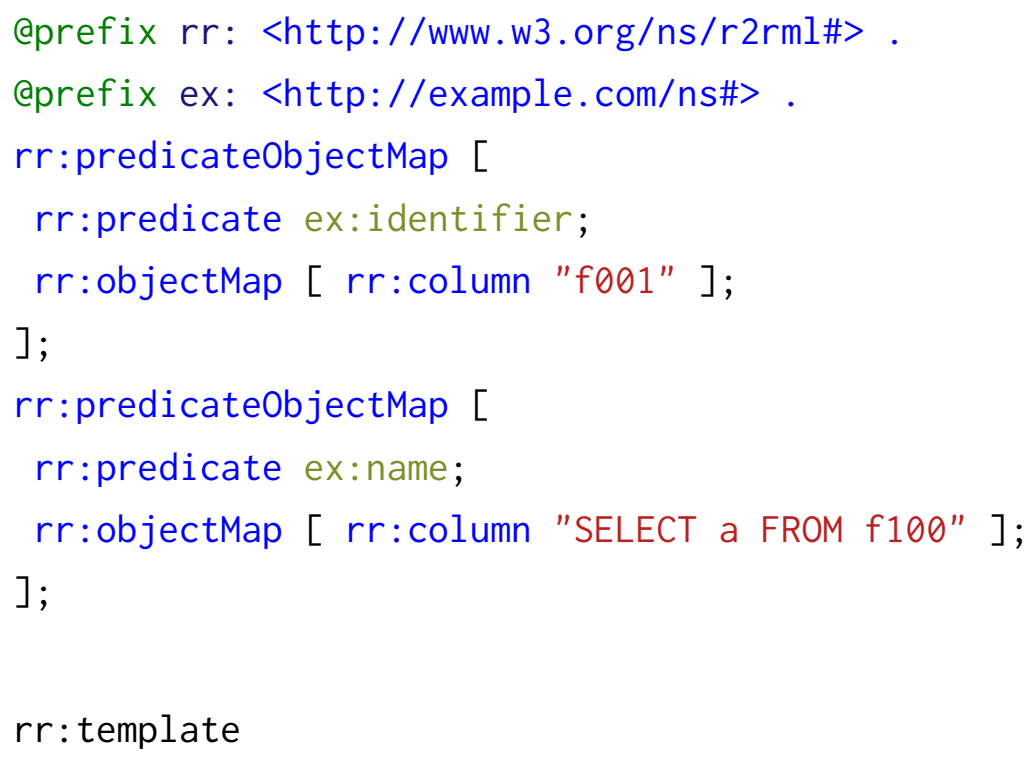

In the R2RML specification a template, $r r$ : template, defines a template-valued term map. The value of $r r$ : template must be a valid string template. A string template is a string that can be used to build strings from multiple components. This template can reference column names by enclosing them in curly braces (i.e., "\{" and "\}").

In marimba-rml and analogously to the interpretation of the values of $r r$ : column, a column name defined in the template must be a valid SELECT query over the relations produced in the R2RML view, with the additional restriction of returning a singlevalued result.

Example 4.5.3 (R2RML templates to describe persons) We now want to map some authority fields and subfields to datatype properties using template-valued term maps. We can define two queries to select an atomic attribute (e.g., field 001) and an atomic attribute within a nested relation (e.g., subfield a of field 100) as follows 
Listing 4.9: marimba-rml templates for properties of person

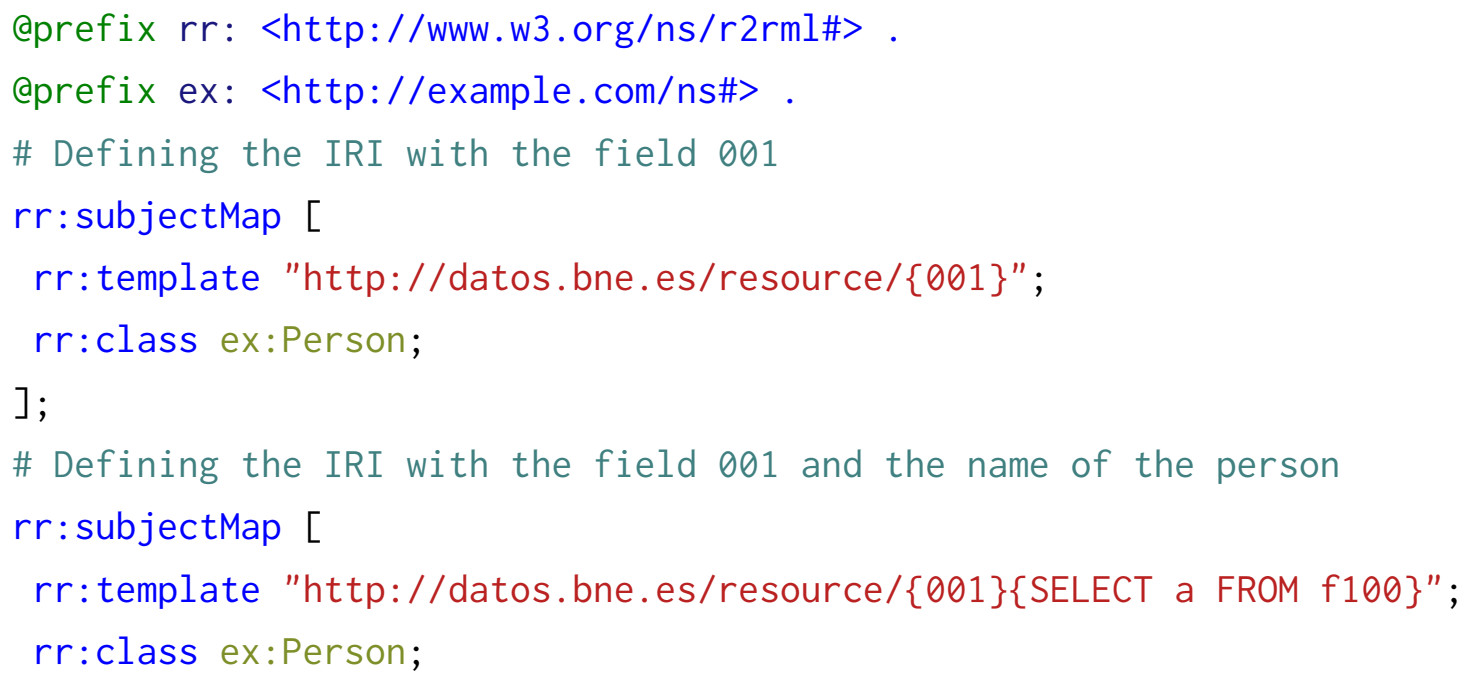

In the R2RML specification, a referencing object map ( $r$ : RefObjectMap) enables relationships to be established between two different Triples Maps. In particular, a referencing object map can be used within a Predicate Object Map to create the objects using the subjects of another Triples Map called the parent Triples Map. A referencing object is defined through: $i$ ) exactly one $r r$ :parentTriplesMap property, whose value must be a Triples Map, and ii) zero or more $r r$ : joinCondi tion properties, whose values must be join conditions. If the child and parent query generating their respective logical tables are not identical, then the referencing object must have a least one join condition. In marimba-rml, we keep the same rules as in the R2RML recommendation but extend the possibilities of the Join Condition to account for nested relations.

\section{rr:joinCondition}

A join condition is further defined by the following properties: $i$ ) a child column ( $r$ : child) that must exist in the logical table of the Triples Map that contains the referencing object map, ii) a parent column ( $r r$ :parent) that must exist in the logical table of the parent Triples Map. The value of these properties are used by the processor to build the joint query that generates RDF triples corresponding to the evaluation of the Referencing object map. 
In marimba-rml, we allow join conditions to be declared using marimba-sql queries, following the orthogonality principle applied to column-valued and template-valued term maps. Moreover, as the join conditions can now themselves be relations, we introduce a new comparison property in marimba-rml, marimba: comparator, which we describe in the next section.

marimba: comparator

In marimba-rml, we allow the use of an additional operator available in marimbasql, SUBSET OF (see Section 4.4). To this end, we introduce a property to specify the comparison operator beyond the equality operator. Nevertheless, the new property is optional and equality is the default comparator. The new property can take the value SUBSET OF. In the next section, we explain the method to build the joint query using the $r$ : joinCondition and the new marimba: comparator property.

\subsection{2 marimba-rml processor}

In this section we describe the general proccess for the generation of RDF triples from a Triples Map. The general process is based on Section 11.1 of the W3C R2RML recommendation and defines the operational semantics of marimba-rml. The main differences of our approach with regards to the original recommendation are:

R2RML views. The results from the evaluation of the marimba-sql query defining the R2RML vi ew correspond to tuples potentially containing nested relations as well as atomic-values.

R2RML Term Maps. The method to evaluate Term Maps to deal with nested relations, which we will be described in Section 4.5.3.

For the sake of completeness, we define below the complete algorithm for generating RDF triples based on the W3C recommendation and our extensions:

1. Let $s m$ be the Subject Map of the Triples Map $t m$.

2. Let $T$ be the tuples resulting from the evaluation of the marimba-sql query of the Logical Table of the Triples Map.

3. Let classes be the Class IRIs of sm.

4. Let $s g m$ be the set of Graph Maps of $s m$. 
5. For each tuple $t \in T$ :

a) Let subject be the generated RDF Term that results from applying $s m$ to $t$

b) Let subjectGraphs be the set of generated RDF Terms that result from applying each Graph map in $s g m$ to $t$.

c) For each class in classes, add triples to the output dataset as follows:

$$
\begin{gathered}
\text { (subject,rdf : type,class, } \\
\text { subjectGraphs } \mid r r: \text { defaultGraph) }
\end{gathered}
$$

d) For each Predicate-object Map pom $\in t m$ :

i. Let predicates be the set of generated RDF Terms that result from applying each of the Predicate maps $p m$ of pom to $t$.

ii. Let objects be the set of generated RDF Terms that result from applying each of the Object maps om of pom to $t$. (This step excludes Referencing Object Maps).

iii. Let pogm be the set of Graph Maps of pom.

iv. Let predicateObjectGraphs be the set of generated RDF terms that result from applying each Graph Map in pogm to $t$.

v. For each possible combination $<$ predicate, object $>$ where predicate $\epsilon$ predicates and object $\in$ objects, add triples to the output dataset as follows:

$$
\begin{gathered}
\text { (subject, predicate, object, } \\
\text { subjectGraphs } \cup \text { predicateObjectGraphs } \mid r r: \text { defaultGraph) }
\end{gathered}
$$

e) For each Referencing Object Map rom of a Predicate-object Map pom of $t m$ :

i. Let psm be the Subject Map of the Parent Triples Map of rom.

ii. Let pogm be the set of Graph Maps of pom.

iii. Let $n$ be the number of attributes in the Logical Table of $t m$.

iv. Let $T$ be the resulting tuples of after evaluating the Joint marimbasql query of rom.

v. For each $t \in T$ :

A. Let childRow be the Logical Table derived by taking the first $n$ attributes of $t$. 
B. Let parentRow be the Logical Table derived by taking all but the first $n$ attributes of $t$.

C. Let subject be the generated RDF Term that results from applying $s m$ to childRow.

D. Let predicates be the set of generated RDF Terms that result each of the Predicate Maps of pom to childRow.

E. Let object be the generated RDF Term that results from applying psm to parentRow.

F. Let subjectGraphs be the set of generated RDF Terms that result from applying each Graph map of sgm to childRow.

G. Let predicateObjectGraphs be the set of generated RDF Terms that result from applying each Graph map of pogm to childRow.

H. For each predicate $\in$ predicates, add triples to the output dataset as follows:

$$
\begin{gathered}
\text { (subject, predicate, object, } \\
\text { subjectGraphs } \cup \text { predicateObjectGraphs) }
\end{gathered}
$$

\subsubsection{Evaluation of Term Maps: the Generated RDF Term of a Term Map}

In this section we describe the expected behaviour of the evaluation of Term Maps by the mapping processor described in the previous section. As defined in the R2RML specification, a Term Map is a function that generates an RDF Term from a logical table row. The result of that function can be:

1. Empty, if any of the referenced columns of the term map has a NULL value.

2. An RDF Term.

3. An error.

In marimba-rml, we apply the same rules as in the original specification but extend its behaviour. As we deal with nested values and possibly several data instances for an attribute, the function of a Term Map can generate a set of RDF Terms, corresponding to the instances of the attribute. More specifically, as described in Section 4.5.1, our extension modifies the processing mechanism of Column-valued Term Maps, and Template-valued Term Maps. 
Column-valued Term Maps

Recall that we allow the specification of a SELECT query inside the $r r$ : column. This query must be a valid marimba-sql query with the following characteristics:

1. It is interpreted as a subquery applied to the relations obtained by applying the rr:sqlQuery property defined in the Logical Table of the parent Triple Map. The parent Triple Map is the triple map where Column-valued Term Map is defined.

2. It returns zero or more values corresponding to exactly one atomic attribute (i.e., column). We allow the query to produce more than one result in order to deal with repeated fields, that are understood by the processor as instances of a nested relation. Then, foreach result value the processor creates one RDF Term.

Template-valued Term Maps

The evaluation of Template-valued Term Maps is equivalent to the one for Columnvalued Term Maps, with one exception:

1. If term type of the Template-valued Term Map is an $r r$ :IRI. The execution of the query should produce one and only one value. Otherwise, the processor will use the first result value. This characteristic of the processor is intended to avoid the generation of duplicated IRIs.

\subsubsection{Building the joint query}

As described in the previous section, evaluation of the Reference object maps results in a joint query that is evaluated to generate RDF triples. In marimba-rml, a Joint marimba-sql query is built according to the following rules.

Undefined join condition When no join condition is defined, the processor builds a joint query of the form:

SELECT * FROM ((child-query)) AS tmp

where (child-query) corresponds to the value of $r r$ : sqlQuery in the logical table of the current Triples Map. 
With join condition When the property $r r$ :joinCondition is defined and complies with the validation requirements, the processor builds the a joint query of the form:

\section{SELECT ALL}

FROM (child-query) AS child, (parent-query) AS parent

WHERE child.(child-column1)(comparator)parent. (parent-column1) AND child.(child-column2) (comparator)parent. (parent-column2) AND ...

where (child-query) and (parent-query) correspond to the values of queries in the logical tables of the current Triples Map and the parent Triples Map respectively. Each (child-column( $n)$ ) and (parent-column $(n)$ ) correspond to each of the join condition pairs.

As a straightfoward example, imagine that we want to generate RDF triples establishing relations between the contributors of a bibliographic resource (indicated in the field 700) and the bibliographic resources. Then, we can define two Triples maps, one for bibliographic resources (Bibliographic) and one for contributors (Contributors). In this way, the Bibliographic Triples Map can be referenced as Parent Triples Map inside a referencing object map of the Contributors Triples Map. We illustrate this in the mappings below:

Listing 4.10: Creating a referencing object map for contributors in marimba-rml

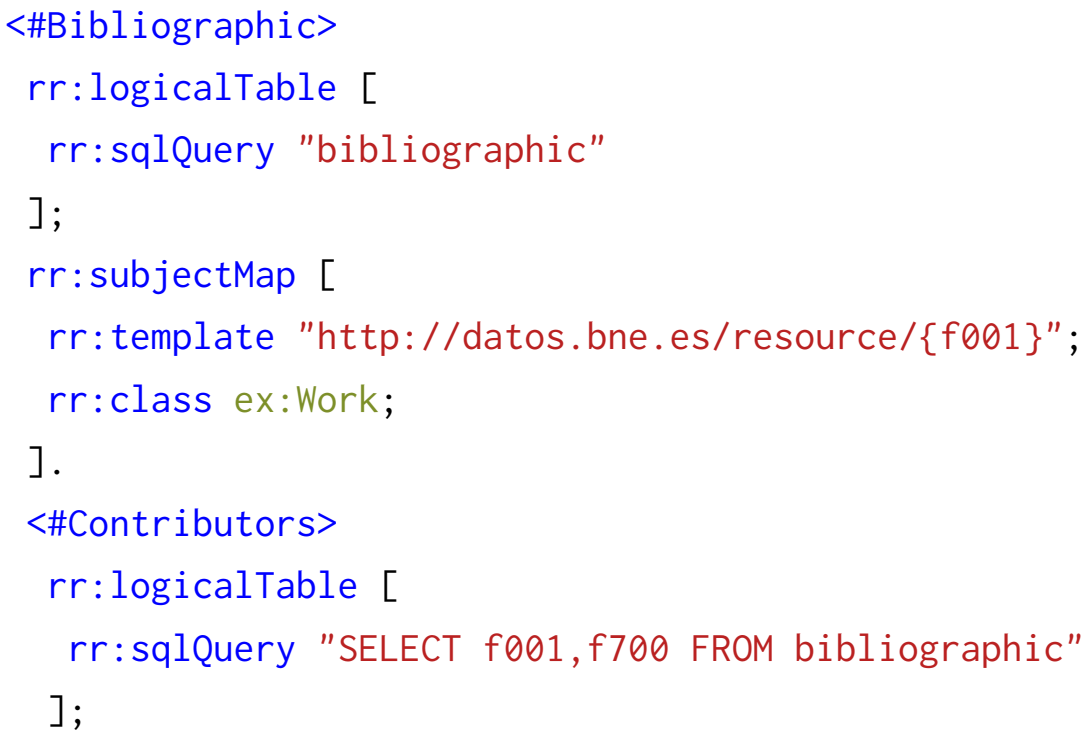




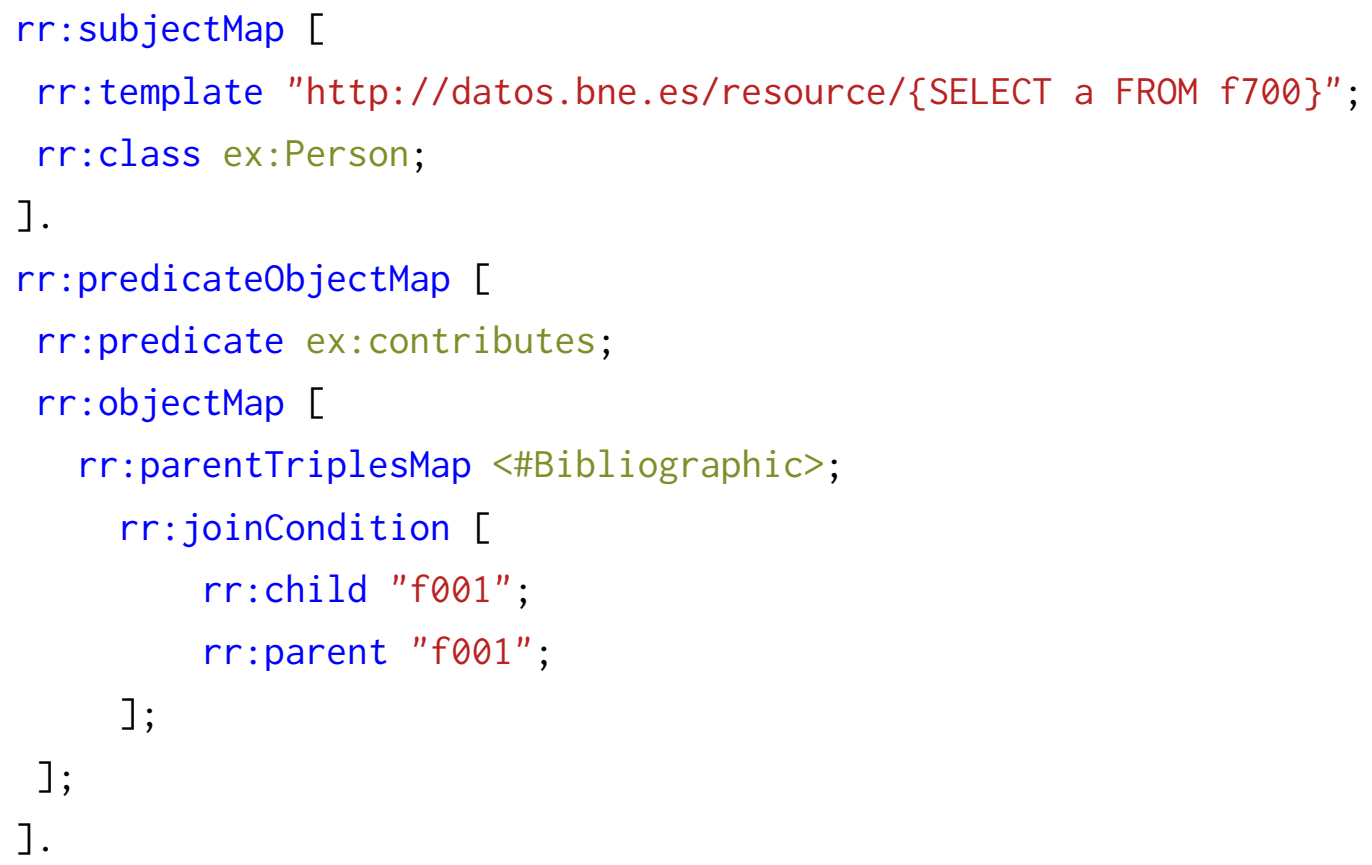

From the example above, the Predicate object map is evaluated and generates the following joint marimba-sql query:

Listing 4.11: Query corresponding to the predicate object map of Example 4.10

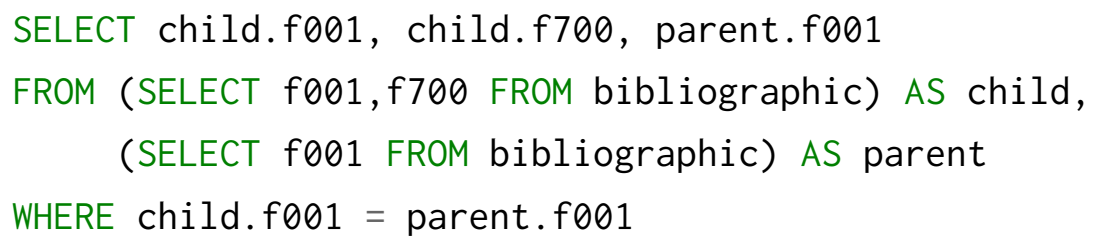

As a more complex example, imagine that we want to generate RDF triples establishing relations between the persons and works of different authority records. It is frequently the case that the authorship relationship is implicit in the authority records corresponding to works. More specifically, the main access point of a work (e.g., field 100) contains the author properties (e.g., the name and the dates in subfields a and d respectively) as well as additional title information (e.g., the subfield $t$ ). In this way, we can define two Triples Maps, one for persons (Persons) and one for works (Works). In this complex case, we can benefit from the operator for comparing relations in marimba-sql: SUBSET OF. Therefore, the Works Triples Map can be referenced as Parent Triples Map inside a referencing object map of the Persons Triples Map but in this case using a special comparator defined by the marimba: comparator property. We illustrate this in the example below: 
Listing 4.12: Creating a referencing object map for creators

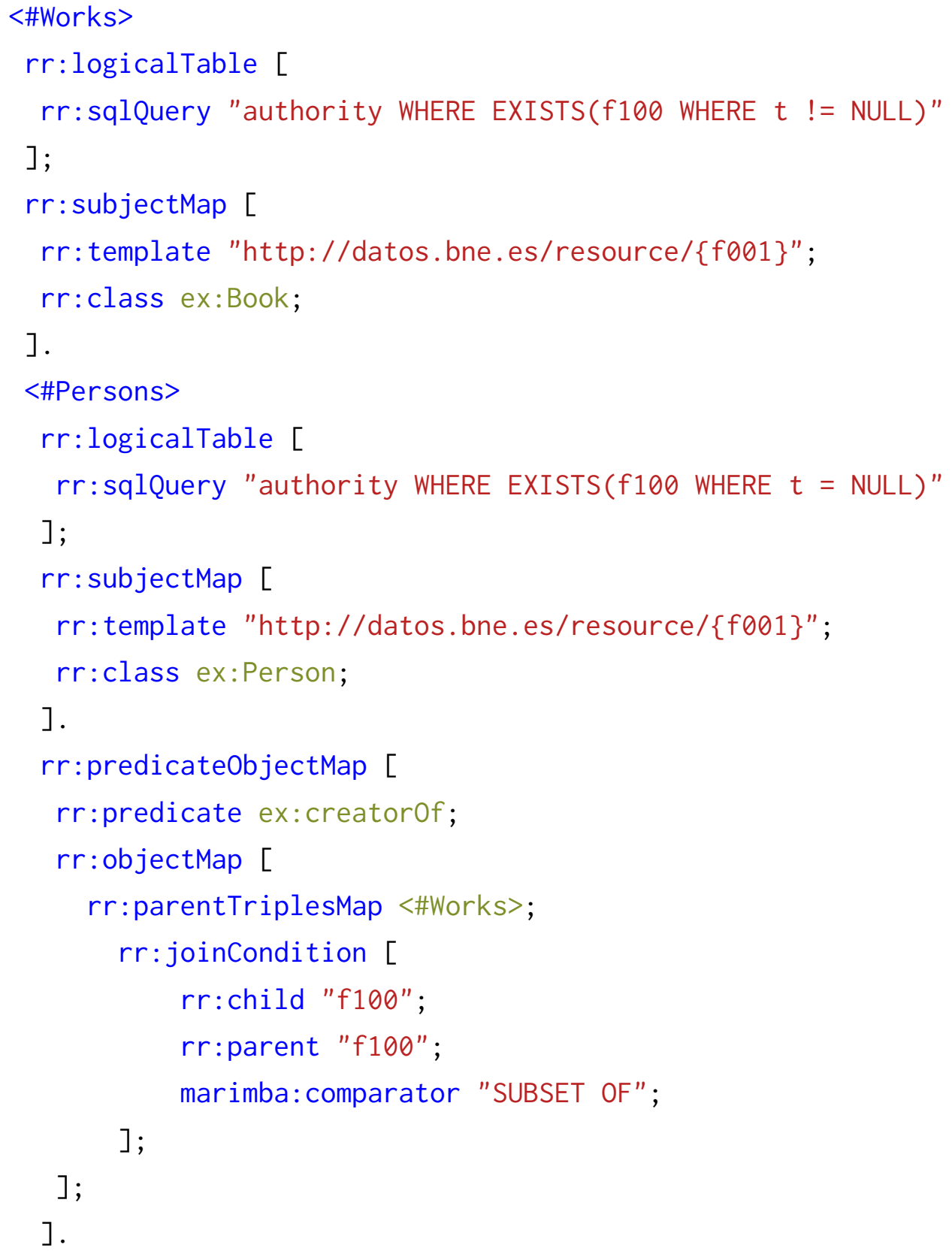

From the example above, the Predicate object map is evaluated and generates the following joint marimba-sql query:

Listing 4.13: Query corresponding to the predicate object map of Example 4.12

SELECT ALL

FROM (authority WHERE EXISTS(f100 WHERE $t=$ NULL)) AS child, 
(authority WHERE EXISTS(f100 WHERE $t \quad !=$ NULL)) AS parent WHERE child.f100 SUBSET OF parent.f100

\subsection{Mapping template generation}

This method utilizes the schema extracted from the source catalogue to create templates that provide structured feedback to experts participating in the mapping process. This method uses the extracted schema $\mathcal{S}$ and record-level statistics $\mathcal{R}$ to present the metadata usage patterns in a way that is useful for experts defining the mapping rules. The ouput of this activity is a set of mapping template documents that we note as $\mathcal{D}$. This method provides a collaborative mechanism for library and ontology experts to define the mapping rules. In this section, we discuss the main features of this method. Each of the mapping rules defined in these templates can be translated into documents defined in the marimba-rml mapping language.

\subsubsection{Mapping templates}

The mapping template generation method generates three mapping templates: $i$ ) classification, $i$ i) annotation, and iii) relation extraction mapping templates, that are described as follows.

Classification mapping template - Unlike other works from the literature (Manguinhas et al. [2010], Aalberg et al. [2006]; Aalberg [2006]) and similarly to Simon et al. [2013], our method transforms authority and bibliographic records. Currently, our method to extract one and only one entity per record. In this way, the entity classification step consists in classifying each bibliographic and authority record into one or several target classes from an ontology, or, in other words assigning one or several $r d f$ : type statements to the resource created from the record. Thus, to support this step we need to provide the domain experts with information about relevant features of the record so they can choose the class of the resource.

Annotation mapping template - Most of the works in the literature include the entity annotation step (also referred to as the property definition step). The entity annotation step consists in mapping fields and subfields to ontology properties. Thus, to support this step we need to provide the experts with information 
about the possible combinations, found in the source catalogue, of fields, subfields, indicators, etc.

Relation extraction mapping template - Relation extraction is probably the most challenging step. It consists in extracting and defining the relationships between the resources that have been created in the previous steps. The challenge is how to identify this relationships within the field and subfields of records. Therefore, to support this step we need to present the experts with valuable patterns found in the source catalogue so that relationships can be found in the source records.

\subsubsection{Templates structure}

In this section, we describe the format and structure of the three mapping templates generated by the template generation method. In order to make the templates usable for experts, we avoid highly technical formats or languages such as XML, XSLT, XQUERY, or Python. Similarly to Sarkar [2015], we argue that non-technical experts are likely to be familiar with tabular formats for data manipulation, in particular with spreadsheets, and that such kind of interfaces can enable experts to define complex mapping rules in a relatively easy way. Therefore, the preferred mechanism for presenting mapping templates are spreadsheets. However, it is worth noting that our method is general and other possibilities such as ad-hoc graphical user interfaces can be built. We describe each of the templates below.

\section{Classification mapping template}

The structure of the classification mapping template, shown in Figure 4.9, consists of three columns: i) MARC 21 metadata; ii) Record count; and iii) Class IRIs. The MARC 21 metadata column contains the field and subfields patterns for the main access point of MARC 21 Authority records, thus the fields in the range 1XX. The Record count presents the number of records in which the MARC 21 metadata pattern was found. The Class IRIs column (coloured/grey column) is used by library experts to define the target class or classes that will be assigned to records presenting the pattern defined in the MARC 21 metadata column. Multiple classes can be assigned by providing a list of IRIs separated by commas. We will explain in Chapter 5, the methodology for assigning values to this column during the mapping and ontology development processes. In Figure 4.9, the black and white columns indicate the information extracted by the 
marimba-mapping method, and the coloured/grey column indicates the information introduced by library experts during the mapping and ontology design processes. The information in the classification template is translated into the marimba-rml queries defining the logical tables of the subject maps in the marimba-rml mappings.

\begin{tabular}{|c|c|c|}
\hline MARC 21 metadata & Record count & Class IRI \\
\hline $100 \mathrm{adt}$ & $1,222,400$ & \\
\hline $100 \mathrm{ad}$ & 999,789 & \\
\hline $100 \mathrm{adtl}$ & 567,534 & \\
\hline $100 \mathrm{ae}$ & 1,658 & \\
\hline $100 \mathrm{ac}$ & 20,768 & \\
\hline
\end{tabular}

Figure 4.9: Classification mapping template

\section{Annotation mapping template}

The structure of the annotation mapping template, shown in Figure 4.10, consists of four columns: i) MARC 21 metadata; ii) Record count; iii) Datatype property IRI; and, iv) Domain IRI. The MARC 21 metadata column presents different patterns of MARC 21 control and data fields, as well as subfields and indicators. These patterns can be classified as follows: $i$ ) control field, such as for example the field 001 for the record identifier; ii) data field, such as for example the field 100 for the author information; and iii) data field and subfield, such as for example the combination $100 a$ for the name of the author. The Record count presents the number of records in which the MARC 21 metadata pattern was found. The Datatype property IRI column is used by the library experts to define the target property or properties that will be assigned to values of the selected pattern variants defined above for the MARC 21 metadata column. The Domain IRI column is used by the library experts to restrict the application of the mapping to resources of an specific type, or in other words, the RDFS domain of the property indicated in the Datatype property IRI column. In Figure 4.10, the black and white columns indicate the information extracted by the marimba-mapping method, and the coloured columns indicate the information introduced by library experts during the mapping process. The information in the annotation template is translated into the marimba-rml queries defining the predicate object maps in the marimba-rml mappings. 


\begin{tabular}{|c|c|c|c|}
\hline MARC 21 metadata & Record count & $\begin{array}{c}\text { Datatype } \\
\text { property IRI }\end{array}$ & Domain IRI \\
\hline $245 n$ & 542,34 & & \\
\hline $321 a$ & 9,589 & & \\
\hline $110 a$ & 63,454 & & \\
\hline $400 a$ & 10,581 & & \\
\hline
\end{tabular}

Figure 4.10: Annotation mapping template

\section{Relation extraction mapping template}

The structure of the relation extraction mapping template, shown in Figure 4.11, consists of five columns: i) MARC 21 metadata; ii) Record count; iii) Object property IRI; iv) Inverse property IRI; and, v) Domain IRI. The MARC 21 metadata indicates the variation of subfields in the main access point fields (i.e., 1XX) found for every pair of MARC 21 authority records. This pattern indicates the latent relationship between a pair of authority records. For example, a record describing an author with the fields 100ad, will eventually have an authorship relationship with other record that contains the same information for the fields 100 ad but includes a subfield $t$ to define the title of a work. This type of relationship is represented with the mapping rule that uses the SUBSET OF comparator described in Listing 4.12. The Object Property IRI column is used by library experts to define the target object property that will be assigned to values of the selected pattern. The Domain IRI column is used by library experts to restrict the application of the mapping to resources of an specific type, or in other words, the RDFS domain of the object property indicated in the Object Property IRI column. The Inverse Property IRI column is optional and is used by library experts to indicate the inverse relationship to be generated during the transformation process. In Figure 4.11, the black and white columns indicate the information extracted by the marimba-mapping method, and the coloured/grey columns indicate the information introduced by library experts during the mapping process. The information in the relation extraction template is translated into the marimba-rml queries defining the referencing object maps in the marimba-rml mappings.

Finally, it is worth noting that more complex marimba-rml mappings can be written independently from these mapping templates, and added to the marimba-rml mappings translated using the templates. 


\begin{tabular}{|c|c|c|c|c|}
\hline $\begin{array}{c}\text { MARC 21 } \\
\text { metadata }\end{array}$ & Record count & $\begin{array}{c}\text { Object property } \\
\text { IRI }\end{array}$ & $\begin{array}{c}\text { Inverse } \\
\text { property IRI }\end{array}$ & Domain IRI \\
\hline $\mathrm{t}$ & 132,541 & & & \\
\hline $\mathrm{I}$ & 57,959 & & & \\
\hline $\mathrm{n}$ & 5,454 & & & \\
\hline
\end{tabular}

Figure 4.11: Relation extraction mapping template

\subsection{Summary}

In this chapter, we have presented several constructs, models and methods to enable the mapping of library catalogues into RDF using ontologies. First, we have proposed the marimba-datamodel, a formal framework based on the Nested Relational Model that can be used to model library data. We have also introduced a recursive algebra that can operate with data in the Nested Relational Model. Moreover, we have introduced a novel method for extracting the nested relational schema of library catalogue sources.

Further, based on the recursive algebra, we have defined a minimal query language marimba-sql that can operate with data in the Nested Relational Model. By combining this query language and our extension of R2RML, marimba-rml we have defined a mapping language that can benefit from the expressive power of both the NRM, and the recursive nested relational algebra. This language overcomes the limitations of languages such as RML or $\mathrm{xR} 2 \mathrm{RML}$ with respect to avoiding ad-hoc iteration mechanisms in the mapping rules, and provides the ability of performing complex queries, including joins. It is worth noting that our main objective is to transform library data sources into RDF not into the marimba-datamodel, which is used for representing library records so that they are processed using declarative and machine-readable mappings. $^{9}$

Finally, using the extracted schema, we have introduced a method to build mapping templates that can be used by library experts to define the mapping rules and build an ontological framework. In the next chapter, we present our contribution for facilitating the ontological engineering process to library experts.

\footnotetext{
${ }^{9}$ In Chapter 7, we describe our current technical implementation of the marimba-rml processor.
} 



\section{Chapter}

\section{Ontology development}

In the context of this thesis, ontology development refers to the process of designing and implementing an ontology network to transform MARC 21 data sources into $\mathrm{RDF}$. As introduced in Chapter 3, the ontology development process makes use of the mapping templates provided by the mapping template generation method described in Section 4.6. These mapping templates are used by domain experts to map the metadata elements of the MARC 21 data sources into RDF using a selection of ontological resources. To this end, the use of the mapping templates by domain experts is directly integrated in the ontology development life-cycle that we describe in this chapter.

This chapter deals with our novel contributions for building library ontology networks within the marimba-framework. These contributions are the following: $i$ ) the extension of existing ontological engineering for the development of library ontology networks with the active participation of domain experts; and $i i$ ) the development and evaluation of a library ontology network, the BNE ontology of the National Library of Spain. In this chapter, we tackle our second open research problem (P2) and in particular the following research question: How can we facilitate the participation of library experts in the modelling and ontology development processes?. To this end, the goal of this chapter is to address our third hypothesis (H3), which stated that analytical data and the feedback of library experts can be used for mapping library catalogues into RDF to develop a library ontology with sufficient quality with respect to state of the art quality metrics.

The rest of the chapter is organized as follows. In Section 5.1, we briefly introduce our contributions for the development of library ontology networks within the marimba-framework. Then, from Section 5.3 to Section 5.6, we describe the application of our contributions to design and develop the BNE ontology, the library 
ontology network for the datos.bne.es project of the National Library of Spain.

\subsection{Contributions}

In this section, we introduce our contribution, marimba-modelling, for the development and publication of library ontology networks within the marimba-framework. This contribution tackles two open research challenges discussed in Section 2.4.5: knowledge acquisition and requirement elicitation and active participation of domain experts. To this end, we propose an iterative and incremental ontology life-cycle model based on the $\mathrm{NeOn}$ methodology and composed of four phases: initialization phase, ontology reuse phase, merging and localization phase, and evaluation and publication phase. The first two phases (initialization and ontology reuse) are mandatory and produce an ontology by directly reusing terms from existing library ontologies. The last two phases (merging and localization and evaluation and publication) may be carried out in later stages of the project. The contributions of this chapter encompass the following novelties with respect to existing ontological engineering methodologies:

1. The elicitation and evolution of requirements and terminology directly from library data sources to support an iterative-incremental process. The elicitation of requirements and terminology are supported by the mapping templates described in Section 4.6.

2. The active participation of domain experts in the ontology design process. marimba-modelling enables the active participation of domain experts in the ontology development process by introducing the requirements specification as an iterative-incremental activity that is performed by the domain experts using the mapping templates generated by marimba-mapping.

3. The definition of a new activity called ontology publication. The ontology publication activity aims at ensuring the publication on the Web of the developed ontology network, following the linked data principles.

\subsubsection{Elicitation and evolution of requirements}

Suárez-Figueroa et al. [2009] proposed a template-based elicitation of requirements which results in an Ontology Requirements Specification Document (ORSD), and defined a set of prescriptive guidelines for this activity. In order to build this document, SuárezFigueroa et al. [2009] proposed eight sequential tasks. The first three tasks are related 
to the identification of the purpose, scope, implementation, intended end-users, and intended uses of the ontology. The rest of the tasks (Tasks 4 to 8) of Suárez-Figueroa et al. [2009] deal with the definition and management of ontology requirements. As we argue in this section, the application of the marimba-framework for ontology development has direct implications on the mechanisms and guidelines for the elicitation of requirements and the terminology extraction.

Figure 5.1 depicts the flow of activities proposed in marimba-modelling and the $\mathrm{NeOn}$ methodology for the requirements specification activity. On the left hand side (a), Figure 5.1 shows an overview of the $\mathrm{NeOn}$ ontology requirements specification activity as described by Suárez-Figueroa et al. [2009]. The activities proposed in marimbamodelling are shown on the right hand side (b). The key novelties of the requirements elicitation in marimba-modelling with respect to the NeOn methodology (SuárezFigueroa et al. [2009]) are the following:

1. A general ORSD document is produced during Task 4. The ORSD documents only the general requirements of the ontology, namely: $i)$ purpose; ii) scope; iii) implentation language; iv) intended end-users; v) intended uses; and, vi) nonfunctional requirements. The main rationale for not creating a detailed ORSD is that the functional requirements have been already analyzed and documented in existing standards (e.g., FRBR, BIBFRAME). Thus, the ontology development team can rely on those standards and focus on fine-grained requirements using the mapping templates. We present and discuss the ORSD document produced for datos.bne.es in Section 5.3.

2. The schema extraction and mapping template generation methods are applied to produce a set of mapping templates, which include the core terminology and document the fine-grained functional requirements. Thus, marimbamodelling does not rely directly on competency questions. In the marimbaframework, the terminology is extracted systematically and fully covers the data in the catalogue sources. The mapping templates are produced by marimbamapping and correspond to the main input of Task 5 (Group requirements). The structure of these templates is depicted in Figure 5.2 and we describe their use and structure in Section 5.1.2.

3. The mapping templates are used to group, validate and prioritize the requirements. This process is performed iteratively through the whole ontology development lifecycle. For instance, based on the statistics provided in the map- 
ping templates, the ontology development team can initially discuss and model those properties that will cover more data in the catalogue (e.g., indicated by the number of records).
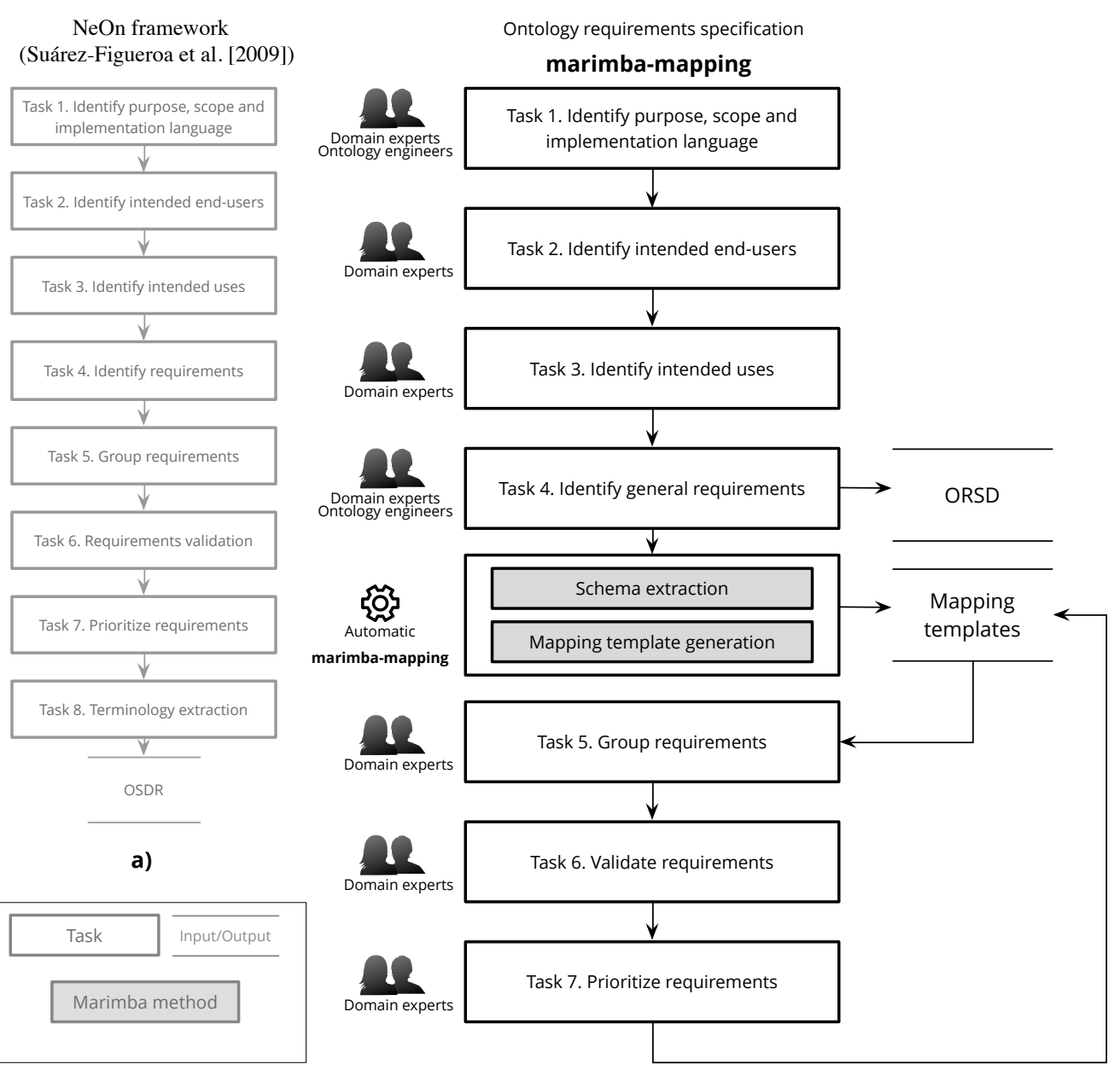

b)

Figure 5.1: marimba-modelling Ontology Requirements specification activity. Adapted from Suárez-Figueroa et al. [2009].

\subsubsection{Active participation of domain experts}

In the marimba-framework, library domain experts directly participate in the mapping process using the mapping templates. The use of mapping templates for mapping MARC 21 data sources into RDF implies defining the classes and properties that are used to model the RDF data to be produced. In this way, domain experts provide the 


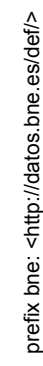
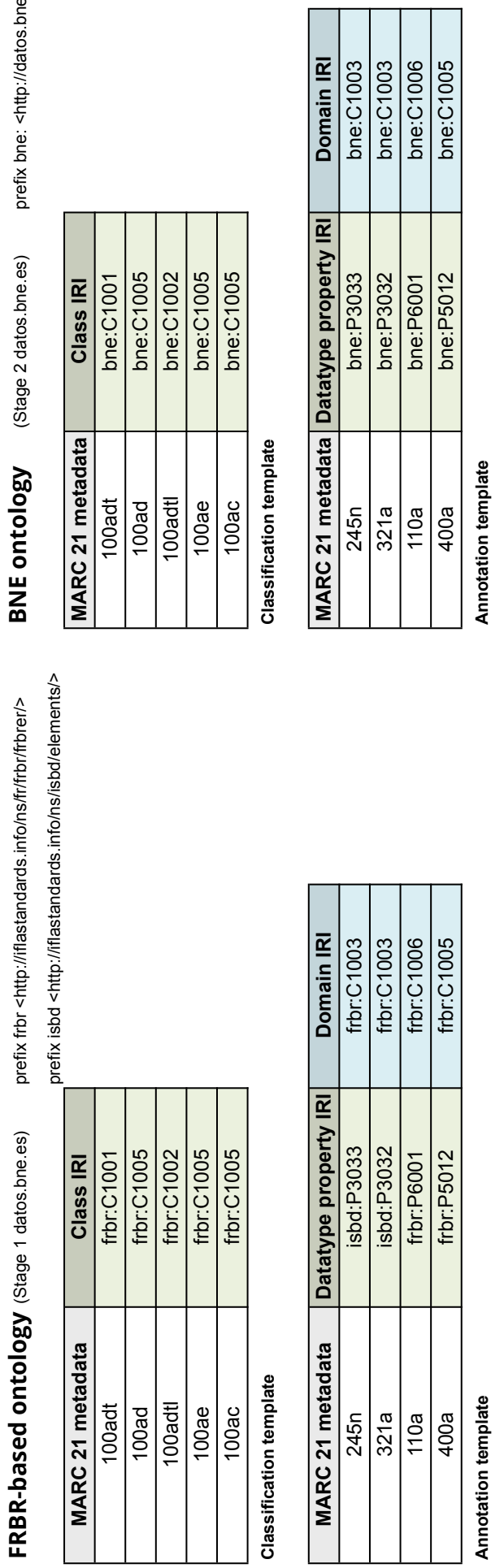

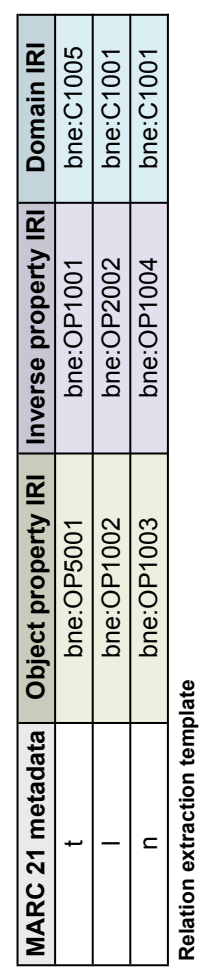

बे.

E

过

D

迆

完

च

동 을

逢

䒕 bo

명 움

范

하욬

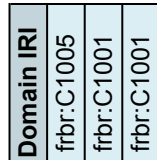

궁

灵

이.

寻

总

\&

范

린.

琼

를

苂

幽

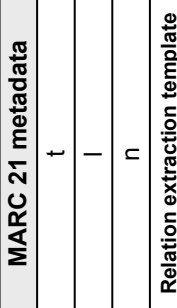

놈

芯

ํ.

i

党 
ontological elements to be used in the mapping and ontology development processes. As described in Section 4.6, the mapping template generation method provides three mapping templates: classification, annotation, and relation extraction. These mapping templates provide information about the use of metadata fields in the MARC 21 data sources. This information facilitates the knowledge acquisition process by enabling domain experts to define the ontological terms to be used, based on the patterns found in the MARC 21 data sources. Each mapping template provides a different input to the ontology development process.

In Figure 5.2, we provide a sample of each type of mapping template used during the development of the FRBR-based ontology (left-hand side of the figure) and the BNE ontology (right-hand side of the figure). Please note that we use IRIs in compact notation (e.g., bne:C1005 and isbd:P3033) and the corresponding prefixes are listed in the figure. For the sake of clarity, we omit the "number of records" column created by marimba-mapping that was shown in the introduction to the templates of Section 4.6.2. The first column of each table (i.e., MARC 21 metadata) in the mapping template is provided by the mapping template generation method, and indicates the use of metadata elements within the MARC 21 data sources. The rest of the columns (i.e., coloured/grey columns) are used by the ontology development team, and particularly the domain experts, to provide the ontology terms and some of their features (e.g., RDFS domain). We explain these mapping templates and their fields below:

Classification template The function of this mapping template is to classify each record into one or several target classes of an ontology. Using this template, the domain experts provide input class IRIs to the ontology development process.

Annotation template The function of this mapping template is to map fields and subfields to ontology properties. Using this template, the domain experts provide input datatype properties and their corresponding RDFS domain to the ontology development process.

Relation extraction template The function of this mapping template is to extract and define the relationships between the ontology instances. Using this template, the domain experts provide input object properties, and their corresponding inverse object properties and RDFS range to the ontology development process. 


\subsubsection{Ontology publication activity}

In this section, we introduce a new activity, ontology publication, that has not been sufficiently covered by existing methodologies. We propose the following definition for the ontology publication activity:

Ontology publication refers to the activity of making available on the Web the machine-readable definition of an ontology or ontology network following the principles of linked data.

The motivation for this activity is the need for enabling machine-readable access through the Web to maximize usability and reusability. This motivation is in line with linked data principles. In fact, together with the growth of Linked Open Data more effort has been made to provide guidelines for ontology publishers (Berrueta and Phipps [2008], Janowicz et al. [2014]). These guidelines complement the ontology publication activity proposed in this section. To introduce this new activity, we follow the documentation procedure defined in the NeOn methodology. Namely, we provide a definition of the process, that can be included in the NeOn glossary, and we create a filling card following the template defined by the NeOn methodology. In Table 5.1, we present the filling card corresponding to the ontology publication activity.

\subsection{Ontology life-cycle model}

marimba-modelling follows an iterative-incremental life-cycle model, which is depicted in Figure 5.3. As shown in the figure, the life-cycle model is composed of four phases:

Phase 1: Initialization - This phase is performed at the beginning of the ontology development process and consists of one core activity, requirements specification, that is part of Scenario 1 of the $\mathrm{NeOn}$ methodology. This phase corresponds to the general requirements specification activity detailed in Section 5.1.1. We discuss the application of this phase during the datos.bne.es project in Section 5.3.

Phase 2: Ontology reuse and design - This phase may be performed during several iterations and includes three core activities: requirements specification, reuse, and design. This phase assumes that a selection of classes and properties from external ontologies is used to directly model the library data sources. As shown in Figure 5.3, the activities of this phase correspond to scenarios 1 and 3 of the $\mathrm{NeOn}$ 


\begin{tabular}{|c|c|}
\hline \multicolumn{2}{|c|}{ Ontology Publication } \\
\hline Definition & $\begin{array}{l}\text { Ontology publication refers to the activity of making } \\
\text { available on the Web the machine-readable definition } \\
\text { of an ontology (network) following the principles of } \\
\text { Linked Data. }\end{array}$ \\
\hline Goal & $\begin{array}{l}\text { The goal of this activity is to make available and ac- } \\
\text { cessible on the Web the code and, ideally, the human- } \\
\text { readable documentation of the ontology (network). }\end{array}$ \\
\hline Input & $\begin{array}{l}\text { The code of the ontology in RDFS and/or OWL. } \\
\text { Optionally, human-oriented documentation describing } \\
\text { core aspects of the ontology such as overview, motiva- } \\
\text { tion, and scope. }\end{array}$ \\
\hline Output & $\begin{array}{l}\text { The ontology is available and dereferenceable under a } \\
\text { stable IRI. Ideally, each ontology term IRI is also avail- } \\
\text { able individually with its IRI. The ontology should be } \\
\text { available at least in one RDF serialization (e.g., Tur- } \\
\text { tle) following linked data best practices (Berrueta and } \\
\text { Phipps [2008]). Optionally, a human-oriented docu- } \\
\text { mentation is available in HTML. }\end{array}$ \\
\hline Who & Ontological engineers and IT administrators. \\
\hline When & $\begin{array}{l}\text { The process should be carried out after the ontology } \\
\text { maintainance activity once the ontology has been im- } \\
\text { plemented in an ontology language. }\end{array}$ \\
\hline
\end{tabular}

Table 5.1: Filling card of the Ontology publication activity

methodology. It is worth noting that this phase does not include the ontology implementation activity. Thus, the outcome of this phase is an ontology formed exclusively by a selection of external classes and properties, in which class and property IRIs are reused directly from external library ontologies. We discuss the application of this phase during the datos.bne.es project in Section 5.4.

Phase 3: Merging and localization - This phase may be performed during several iterations and adds three activities to the second phase: merging, implementation, and localization. This phase also focuses on reuse but includes a merging phase to create a new ontology network, where each class and property is defined 
by a new IRI. This phase includes the processes and activities of the Scenario 5 and 9 of the NeOn methodology. The main idea is that the reused classes, properties and constraints are integrated within an ontology network, owned by the organization developing and publishing the ontology with the marimbaframework. We discuss the application of this phase in Section 5.5.

Phase 4: Evaluation and publication - This phase may be performed during several iterations and consists of the ontology evaluation and publication activities. We discuss the application of this phase during the datos.bne.es project in Section 5.6.

The phases proposed in our life-cycle model can be applied incrementally. Specifically, an ontology development team may execute only the first two phases at early stages of the project, and carry out the third and fourth phases during a second stage of the project. We discuss the implications of these two approaches below:

1. Directly reusing ontological resources (Phase $1+$ Phase 2): Some examples of the use of this approach are the data.bnf.fr and British National Bibliography projects. The main advantages of this approach are its agility and simplicity. However, this approach also presents several disadvantages: $i)$ potential data inconsistencies; and $i i$ ) uncontrolled changes and lack of maintainance of the reused ontologies, which can lead to data that is poorly modelled. Nevertheless, we argue that these phases can be executed during the first iterations of the ontology development project, and later combined with the rest of the phases.

\section{Reusing and merging ontological resources into a new ontology network} (all phases): This approach also focuses on reuse but adds a merging phase to create a new ontology network owned by the organization developing the ontology. As we discuss in this chapter, the benefits of developing an ontology network owned by the organization behind the data and ontology publication are the following: $i$ ) it provides a better identification and removal of ontology inconsistencies; $i i$ ) it improves change and documentation management processes; and, iii) it offers more flexible ontology mantainance, localization, and publication.

In the next section, we describe the application of the proposed life-cycle model for the ontology development process in the datos.bne.es project, and highlight the benefits of applying the above described ontology life-cycle. 


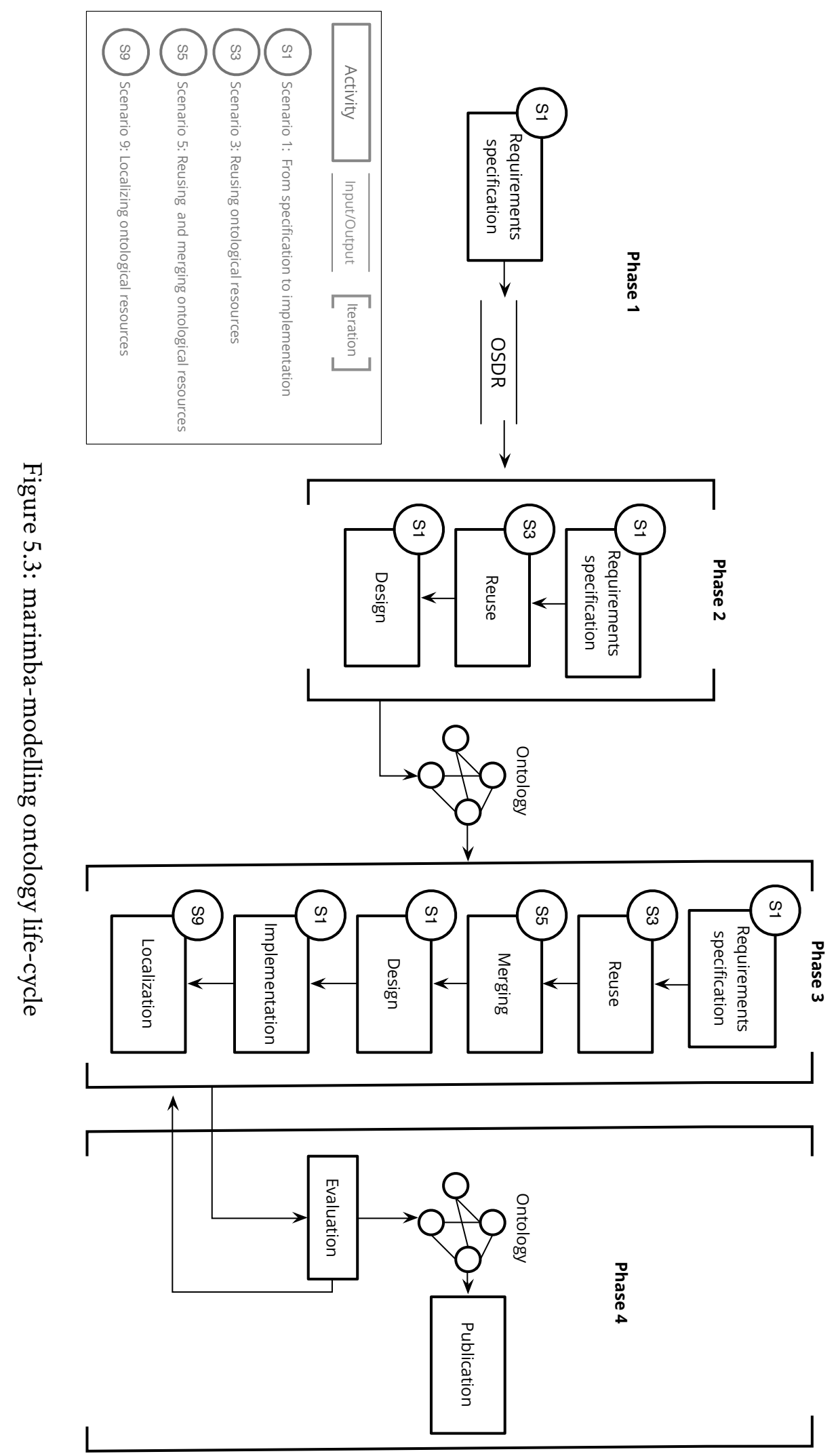




\subsubsection{Ontology life-cycle model for datos.bne.es}

In this section, we briefly introduce the application of the marimba-modelling lifecycle model for the ontology development process in the datos.bne.es project. The ontology development process was carried out into two well defined stages, which produced two distinct ontologies during two different milestones of the datos.bne.es project: the FRBR-based ontology and the BNE ontology. Both stages were carried out in several iterations that incrementally improved and extended the resulting ontologies. In the following, we introduce the key characteristics of the application of the life-cycle model, which is depicted in Figure 5.4.

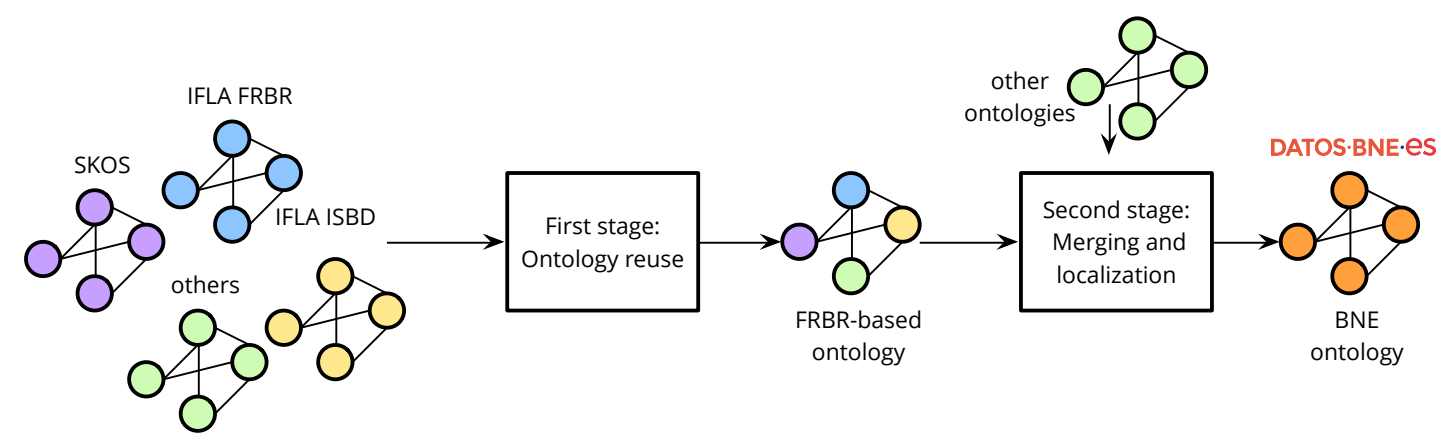

Figure 5.4: Ontology life-cycle model followed during the datos.bne.es project. The first stage included the first and second phases of the life-cycle. The second stage included the first, second, third and fourth phases of the life-cycle.

The first stage consisted in performing phases 1 and 2 of the life-cycle model, namely Initialization, and Ontology reuse. The outcome of this stage was the FRBRbased ontology, an ontology that reused the classes and properties of several library ontologies. The FRBR-based ontology was one of the first direct applications of the IFLA FR and ISBD ontologies to linked data initiatives, as described in Vila-Suero and Escolano [2011]. Additionally, the FRBR-based ontology included classes and properties from the following ontologies: SKOS, IFLA ISBD, IFLA FRAD, IFLA FRSAD, RDA Group Elements 2, RDA Relationships for WEMI, Dublin Core terms, SKOS, and MADS/RDF. We describe the application of this stage for the development of the FRBR-based ontology in Section 5.4

The second stage built on the ontology defined in the previous phase. In this stage, the ontology development team carried out the second, third and fourth phases of the life-cycle model, namely Ontology reuse and design, Merging implementation and localization, Evaluation and publication. The outcome of this stage was the BNE ontology, 
which contains classes and properties defined within the datos.bne.es domain. The BNE ontology is the result of the application of the merging and localization phase and includes alignments with the ontologies that were reused by the FRBR-based ontology developed during the first stage, as well as definitions of ontological elements in Spanish and English. Moreover, the BNE ontology has been made publicly available and documented under a stable URI ${ }^{1}$ by carrying out the ontology publication task introduced in Section 5.1.3 We describe the process of developing, localizing, publishing, and evaluating the BNE ontology, as well as its main features in sections 5.5 and 5.6.

In the remainder of this chapter, we discuss each of the phases proposed by the marimba-modelling life-cycle, their application in datos.bne.es, and discuss the characteristics of the development of the FRBR-based and BNE ontologies.

\subsection{First phase: Initialization}

In this section, we describe the application of the first phase of the marimba-modelling life-cycle. The core activity of this phase is the general requirements specification activity that produces the ORSD document, which will be used during the next phases.

In the datos.bne.es project, this phase was executed at the beginning of the project. The first step was to produce the ORSD documenting the purpose, scope, implementation language, intended end-users, and intended uses of the ontology. Table 5.2 presents an overview of the resulting ORSD.

The next step in this phase consisted in the definitions of the functional and nonfunctional requirements. Following the approach presented in Section 5.1.1, the functional requirements elicitation consisted in analyzing and selecting existing functional requirements for the library domain. The IFLA Functional Requirements for Bibliographic Records standard were used due to their maturity and natural alignment with other ontological frameworks such as RDA. The FRBR standard defines a set of functional requirements to be fulfilled by library data. The scope of the FRBR requirements is similar to the scope of CQs in existing ontology engineering methods. In particular, Section 7.1 of the current text (IFLA [2009]) clearly defines questions that should be answered by library data. More importantly, the bibliographic entities and their properties that enable these questions to be answered are carefully documented in the standard. Finally, IFLA, the standardization body maintaining FRBR,

\footnotetext{
${ }^{1}$ http://datos.bne.es/def/
} 
has recently undertaken the implementation of FRBR in the OWL language, which provided a good basis for developing an ontology for the datos.bne.es project.

The last step consisted in gathering and documenting five high-level non-functional requirements which we discuss below.

\begin{tabular}{|c|c|}
\hline & BNE Ontology ORSD \\
\hline \multirow[t]{2}{*}{1} & Purpose \\
\hline & $\begin{array}{l}\text { The purpose of the BNE Ontology is to a provide an agreed } \\
\text { knowledge model of the catalogue data within BNE that is } \\
\text { aligned to existing bibliographic and library standards. }\end{array}$ \\
\hline \multirow[t]{2}{*}{2} & Scope \\
\hline & $\begin{array}{l}\text { The ontology has to focus on representing BNE catalogue data } \\
\text { and the level of granularity is directly related to the character- } \\
\text { istics of this catalogue data. }\end{array}$ \\
\hline \multirow[t]{2}{*}{3} & Implementation language \\
\hline & The ontology has to be implemented in the OWL language \\
\hline \multirow[t]{3}{*}{4} & Intended End-Users \\
\hline & $\begin{array}{l}\text { User 1. A general user that is searching for information about } \\
\text { specific library resources hosted by BNE. }\end{array}$ \\
\hline & $\begin{array}{l}\text { User 2. A specialized user that is collecting catalogue informa- } \\
\text { tion to reuse it within another catalogue. }\end{array}$ \\
\hline \multirow[t]{3}{*}{5} & Intended Uses \\
\hline & $\begin{array}{l}\text { Use 1. Publish library catalogue data coming from library } \\
\text { records }\end{array}$ \\
\hline & $\begin{array}{l}\text { Use 2. Search library information about authors, organiza- } \\
\text { tions, library resources, subjects, etc. }\end{array}$ \\
\hline
\end{tabular}

Table 5.2: BNE Ontology OSRD summary

Aligning the used terminology with existing library standards (NFR-1). The library community has produced several standards and best practices to promote interoperability and richer data models. Therefore, one key requirement is to align as much 
as possible the terminology used within the ontology with existing standards that are already in place and extensively used to drive the catalogue data model and cataloging processes. In particular, the ontology should be aligned as much as possible with two mature and widely accepted standards to describe the bibliographic domain: ISBD and FRBR.

Reusing existing ontologies (NFR-2) Following the Linked Data approach and best practices, one of our goals is to re-use ontologies already available and extensively used on the Web in order to describe library data. It is important to note, that this requirement is directly influenced by requirement NFR-1, in the sense that ontologies that are based on widely accepted standards are given a higher priority than those not aligned with bibliographic standards.

Including labels and descriptions in Spanish (NFR-3) The National Library of Spain is an important source of cultural resources in the Spanish-speaking world. An important part of the data contained in the catalogue is in Spanish and reused by many other institutions both in Spain and Latin America. Moreover, library standards mentioned in the requirement NFR-1 have been translated into Spanish and serve as reference for cataloging practices. In this context, an important requirement is to provide labels and descriptions in Spanish to serve as a source for other institutions and to align the ontology with current data models and cataloging practices and to enable the addition of the other official languages in future versions of the ontology.

Providing a stable namespace to host the ontology (NFR-4) As stated in Janowicz et al. [2014], Poveda-Villalón et al. [2013], and Atemezing et al. [2013] many of the ontologies available in the Web of Data are often unspecified, unstable, and poorly maintained. This issue has a direct impact on several aspects of the linked data produced using these ontologies. One of these aspects is the usability and understandability of the data, in the sense that it is often limited for the data consumer to access the ontological axioms or even their descriptions. Another aspect is reasoning capabilities over the data, in the sense that if axioms are not accessible and/or change frequently this can lead to unexpected or undesired results. Lastly, another aspect is ontology evolution and versioning, in the sense that the data publisher will eventually evolve the ontology and there are not proper mechanisms to do this when exclusively reusing ontology components that are not under the control of the data publisher. Furthermore, the requirement NFR-3 mandates the addition of labels and descriptions in Spanish 
and this inclusion is limited when working exclusively with ontologies under a namespace not owned by the ontology publisher, the BNE in this case.

Publishing the ontology on the Web by following linked data publication best practices (NFR-5) The publication of ontologies on the Web has been frequently overlooked in the literature over the years. This question becomes even more important when the ontology will directly support the publication of a linked dataset, as it is the case of the BNE ontology. Therefore, the BNE ontology should be made available following linked data and ontology publication best practices.

\subsection{Second phase: Ontology reuse}

As introduced in Section 5.2, this phase of the marimba-modelling life-cycle may be performed during several iterations and includes three core activities: fine-grained requirements specification, reuse, and design. This phase assumes that a selection of classes and properties from external ontologies are used to directly model the library data sources.

The main input of this phase is the ORSD generated by the initialization phase. The output of this phase is an ontology formed by a selection of classes and properties, in which class and property IRIs are reused directly from external library ontologies. As the ontology development team does not produce any formalization of the ontology, the ontology implementation activity from the $\mathrm{NeOn}$ methodology is not included during this phase. The fine-grained requirements specification is managed within the mapping templates described in Section 5.1.2. These mapping templates may be generated several times during the iterations of this phase to reflect changes in the MARC 21 data sources, such as use of new metadata elements to describe bibliographic resources. In the following, we describe the application of this phase during the development of the FRBR-based ontology.

In the FRBR-based ontology, the IRIs of external library ontologies were used directly during the mapping process. We have provided a review of these library ontologies in Section 2.1.4. In particular, the core of the ontology was composed by the classes Manifestation, Work, Person, Expression, and Corporate body from the IFLA FRBR ontology. Furthermore, the class Thema from the FRSAD ontology and the class Concept from SKOS were used to model data describing the subjects of Works. The properties for describing bibliographic data were reused from a number of on- 
tologies, namely IFLA ISBD, RDA Group Elements 2, RDA Relationships for WEMI, Dublin Core terms, SKOS, and MADS/RDF; whereas the properties for describing authority data were reused from the IFLA FRBR, IFLA FRAD, IFLA FRSAD, and RDA Group Elements 2 ontologies. Figure 5.5 depicts the core of the FRBR-based ontology produced during this phase.

This phase did not include the ontology publication activity. Nevertheless, the reused ontologies were loaded into the datos.bne.es SPARQL endpoint, where the linked dataset was hosted. ${ }^{2}$ This approach to ontology publication was limited in the sense that it did not provide a global view of the ontology and its documentation for potential consumers. Moreover, this method did not fulfill the non-functional requirements related to ontology publication and maintainance of the ontology network.

\subsection{Third phase: Ontology merging and localization}

This phase combines the ontology reuse process of Scenario 3 of the NeOn methodology with the ontology merging process of Scenario 5 and the ontology localization activity of Scenario 9. Moreover, this phase includes the ontology implementation activity of Scenario 1 . This phase may be carried out iteratively and incrementally to merge and localize the ontological terms of potentially overlapping and evolving external ontologies.

\subsubsection{Ontology merging}

The ontology merging process is composed of two steps (Suárez-Figueroa et al. [2015]):

1. Ontology alignment: The goal of this step is to obtain a set of alignments among the selected ontological resources.

2. Ontology merging: The goal of this step is to merge the ontological resources using the alignments to obtain a new ontological resource from the aligned resources.

The ontology network designed after the second phase of the life-cycle consists of external set of classes, properties and statements from different ontologies, each of them with external IRIs. When merging is applied, two strategies can be followed: $i$ ) performing the ontology alignment activity and publishing the alignments using a standard

\footnotetext{
${ }^{2}$ http://datos.bne.es/sparql
} 
5.5. Third phase: Ontology merging and localization
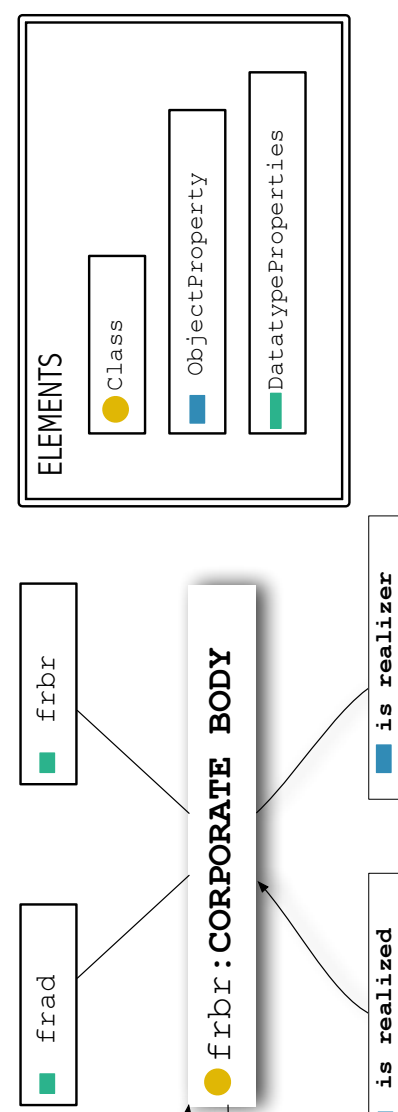
format such as OWL or the Alignment format (David et al. [2011]); and ii) performing the alignment and create new IRIs for each element to merge the external ontological definitions. The advantage of the second strategy is that the developers have more control over the definitions and can more naturally extend, maintain, and document the ontology network.

Ontology merging in datos.bne.es. The goal of the merging process in datos.bne.es was to produce a new ontology network building on the FRBR-based ontology designed during the second phase. The main idea was to create an IRI for every class and property using a stable domain maintained by the BNE. The namespace selected for creating the IRIs was http://datos.bne.es/def/. This namespace follows the recommendation of the "Persistent URIs Report" by the ISA (Interoperability Solutions for European Public Administrations) programme. ${ }^{3}$ The process of creating these IRIs was mainly manual and was carried out by the ontological engineers using the Protegé tool. The merging process was applied to each class and property in the following way:

1. Creating an IRI for the term. The ontology development team created an IRI for each term. Whenever possible, the identifier of the original IRI was reused within the BNE namespace. For example, the Work class from FRBR is frbr: C1001 and was mapped to bne:C1001. The ontology development team used the following IRI conventions: $i$ ) the prefix $C$ was used for classes; $i i)$ the prefix $\mathrm{P}$ was used for data properties; and $i i i$ ) the prefix OP was used for object properties.

2. Including an alignment relation. A relation with the reused term was then added using the following RDFS primitives: the property rdfs: subClassOf for classes; and the property rdfs : subProperty0f for properties. These primitives were used to indicate that the newly generated ontological elements extended external ontological resources.

Following the above process, a minimal ontology network was created to be used as input of the ontology design and implementation activities.

Ontology design and implementation for datos.bne.es. During these two activities, the ontological engineers focused on improving the minimal ontology network pro-

\footnotetext{
${ }^{3}$ https://joinup.ec.europa.eu/community/semic/document/ 10-rules-persistent-uris (Last viewed 3rd May 2016)
} 
duced by the ontology merging process. The ontology development team added different axioms, domain and range restrictions and annotations such as labels. We describe the resulting ontology network in Section 5.5.2.

Ontology localization in datos.bne.es. This activity consisted in adding labels in Spanish to classes and properties. The ontology development team reused the guidelines and previous work of Montiel-Ponsoda et al. [2011], that dealt with translation guidelines for the IFLA ontologies. Specifically, two type of labels were added to every class and property: the rdfs: label annotation property, that was aligned with the library standards (e.g., FRBR), and a bne: label annotation property, which provided a more end-user friendly definition of the terms. For example, the class frbr:C1002 that corresponds to the FRBR Expression class was labelled with the rdfs: label labels "Expression" and "Expresión"; and with the bne : label labels "Version" and "Versión". Moreover, every label was annotated with the corresponding language tag, following the rules defined by the RDF 1.1 recommendation (e.g., "Versión"@es and "Version”@en).

\subsubsection{The BNE ontology}

The BNE ontology is the result of the iterations and phases described in the previous sections. This ontology is used to model the data of the service of datos.bne.es. The BNE ontology reuses the core classes of FRBR (i.e., Person, Corporate Body, Work, Expression, Manifestation, and Item) but incorporates several datatype and object properties ${ }^{4}$ to the model in order to widen the coverage of a large library catalogue like the BNE catalogue. Figure 5.6 shows the core classes and object properties of the BNE ontology where the reader can observe how we relate and interconnect core FRBR concepts.

The ontology is composed of six classes and directly reuses the class Concept from the SKOS vocabulary. As the SKOS vocabulary is a stable standard, the ontology development team did not apply the merging process for the Concept class. Nevertheless, this class is used within the axioms of the BNE ontology as the range of several object properties. Regarding the datatype properties, the ontology network integrates more than $\mathbf{2 0 0}$ datatype properties to describe fine-grained properties of the catalogue resources. The BNE ontology defines 33 object properties, that include the FRBR "Primary" and the "Responsibility" relationships for relating authority and

\footnotetext{
${ }^{4}$ For instance, a direct authorship object property to connect persons and manifestations.
} 
bibliographic data ${ }^{5}$. The full documentation describing each of the classes, datatype properties and object properties is available online. ${ }^{6}$

\subsection{Fourth phase: publication and evaluation}

This phase corresponds to publication and evaluation of the produced ontology. The two activities can be carried out iteratively to improve the quality of the ontology. In this section, we describe these two activities in the context of the datos.bne.es project.

\subsubsection{Ontology publication}

This ontology publication activity, described in Section 5.1.3, was carried out to publish the BNE ontology. Specifically, the ontology development team executed the following steps:

1. Producing an HTML ontology documentation using the tool Widoco ${ }^{7}$. Widoco expects an ontology in OWL/RDFS as input and produces an HTML document by using the ontological definitions, including their axioms, labels and descriptions. The resulting HTML document was revised and extended by the ontology development team. Figure 5.7 presents a screenshot of the HTML documentation that is available online at http://datos.bne.es/def/.

2. Exporting the ontology network in two RDF serializations, RDF/XML and Turtle using the Protegè tool.

3. Configuring a content negotiation mechanism, following the linked data patterns described by Berrueta and Phipps [2008] and in particular the 303 pattern. The content-negotiation mechanism allows the datos.bne.es service to serve three different formats (HTML, RDF/XML or Turtle), depending on the preferences of the user-agent requesting the ontology. For instance, a web browser normally requests the format text/html so the content-negotiation mechanism provides the documentation generated by Widoco in HTML. We explain this content-negotiation mechanism below.

\footnotetext{
${ }^{5}$ We point the reader to Section 2.1.1, where we have introduced "Primary" and the "Responsibility" relationships of FRBR.

${ }^{6}$ http://datos.bne.es/def/

${ }^{7}$ https://github.com/dgarijo/Widoco (Last viewed 12th May 2016)
} 


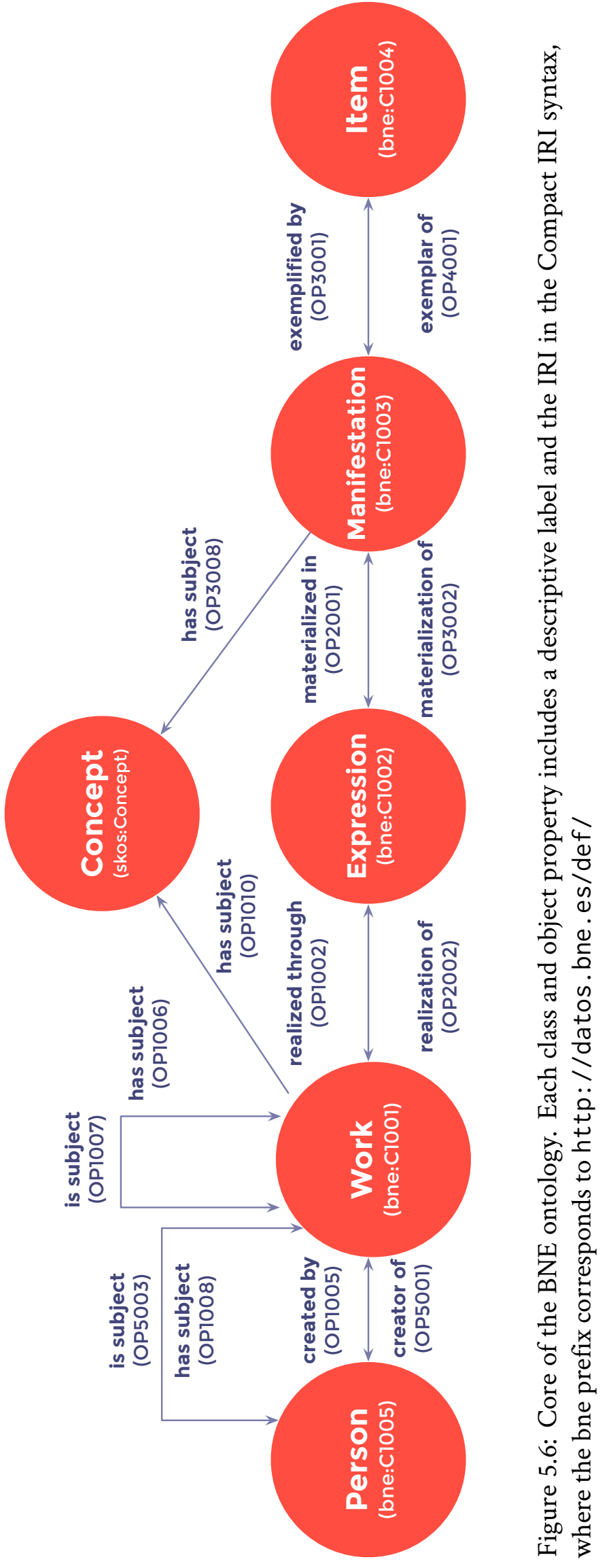


The canonical IRI for the ontology is http://datos.bne.es/def/. Using content negotiation, this canonical IRI is resolved into:

- http://datos.bne.es/def/ontology.html by default and if the user agent requests text/html.

- http://datos.bne.es/def/ontology.ttl when the user agent requests text/turtle.

- http://datos.bne.es/def/ontology.rdf when the user agent requests application/rdf $+x m l$.

\subsubsection{Ontology evaluation}

According to the $\mathrm{NeOn}$ methodology, ontology evaluation refers to the activity of checking the technical quality of an ontology against a frame of reference. A frame of reference can be an specification of requirements, a set of competency questions, or the application of the ontology to a real-world scenario. Ontology evaluation approaches can be classified into five categories:

Assessment by humans The quality of the ontology is assessed by human experts. The human experts define the criteria or requirements to be met by the ontology. Examples of this category are the framework proposed by Gómez-Pérez [1996] and OntoClean by Guarino and Welty [2009].

Application-based The quality of the ontology is measured with respect to its suitability for a specific application or task. Examples of this category are the case study of Fernández et al. [2009], and the task-based approach proposed by Porzel and Malaka [2004].

Topology-based The quality of the ontology is assesed by computing a set of measures based on the internal structure of the ontology. Examples of this category are OOPS! by Poveda-Villalón et al. [2014], the ranking-based approach by Alani et al. [2006], the framework proposed by Duque-Ramos et al. [2010], and OntoQA by Tartir et al. [2005].

Data-driven The ontology is compared against an unstructured resource representing the problem domain. Examples of this category are the approach by Alani et al. [2006] and the approach by Brewster et al. [2004]. 


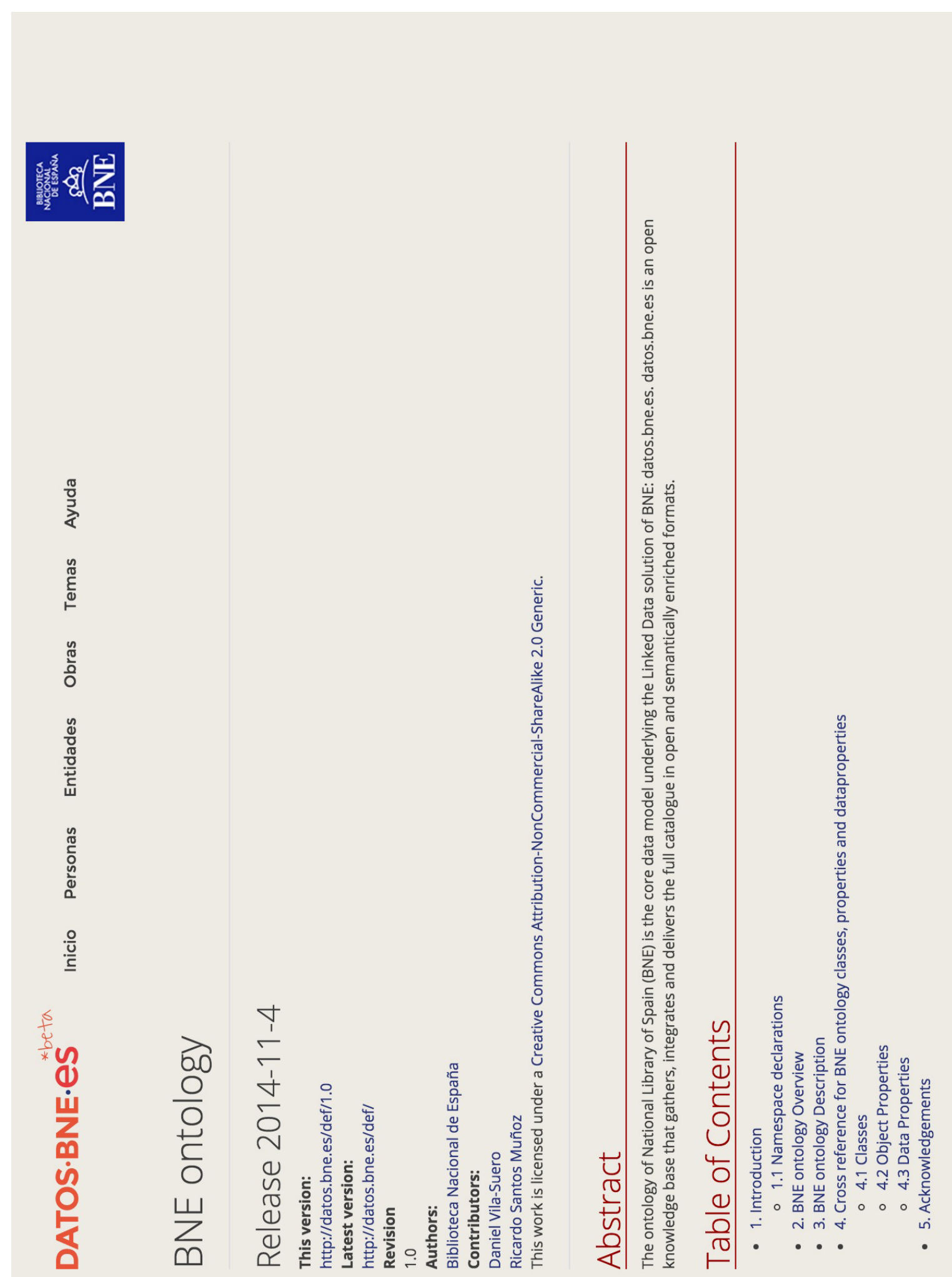


Gold standard The ontology is compared against a structured resource representing the domain of the problem. Examples of this category are the metrics-based approach by Burton-Jones et al. [2005] and the methods proposed by Maedche and Staab [2002].

In this thesis, we evaluate the BNE ontology by performing two types of evaluation: i) an application-based evaluation, based on the task-based experiment which will be described in Chapter 8, and ii) a topology-based evaluation using OOPS!, a state-ofthe-art evaluation tool, which we describe below. OOPS! (OntOlogy Pitfall Scanner!) is an online application for detecting pitfalls in ontologies. The tool is based on a pitfall catalogue that contains 41 pitfalls and the current version of the tool ${ }^{8}$ is able to detect 33 out of the 41 defined pitfalls. The tool defines an indicator for each pitfall according to their possible negative consequences (critical, important, minor). For each pitfall, the tool provides detailed explanatory and provenance information.

\section{BNE Ontology evaluation}

In this section, we describe and discuss the use of OOPS! to evaluate the BNE ontology during two iterations. These iterations consisted in two steps: $i$ ) diagnose, in which the ontology development team used the online version of OOPS! to detect pitfalls in the ontology; and, ii) repair, in which the ontology development team fixed the pitfalls identified by OOPS!.

Figure 5.8 shows a screenshot of the results provided by OOPS! before starting the first diagnose-repair iteration. Below, we discuss the iterations carried out by the ontology development team for evaluating and improving the BNE ontology.

First iteration. As shown in Figure 5.8, the tool detected seven pitfalls (three pitfalls labelled as important and four as minor). The first iteration focused on repairing one of the three pitfalls labelled as important. Below, we describe the pitfalls ${ }^{9}$ and the different actions taken by the ontology development team:

- P08: Missing annotations (Minor). This pitfall indicated the lack of $r d f s$ : comment annotations to describe the ontology classes and properties. The ontology development team agreed not to repair this pitfall because every class and property contains labels both in English and Spanish and the majority of the terms refer

\footnotetext{
${ }^{8}$ oops. linkeddata.es/ (Last viewed 10th April 2016)

${ }^{9}$ We use the alphanumeric codes of the pitfall catalogue.
} 


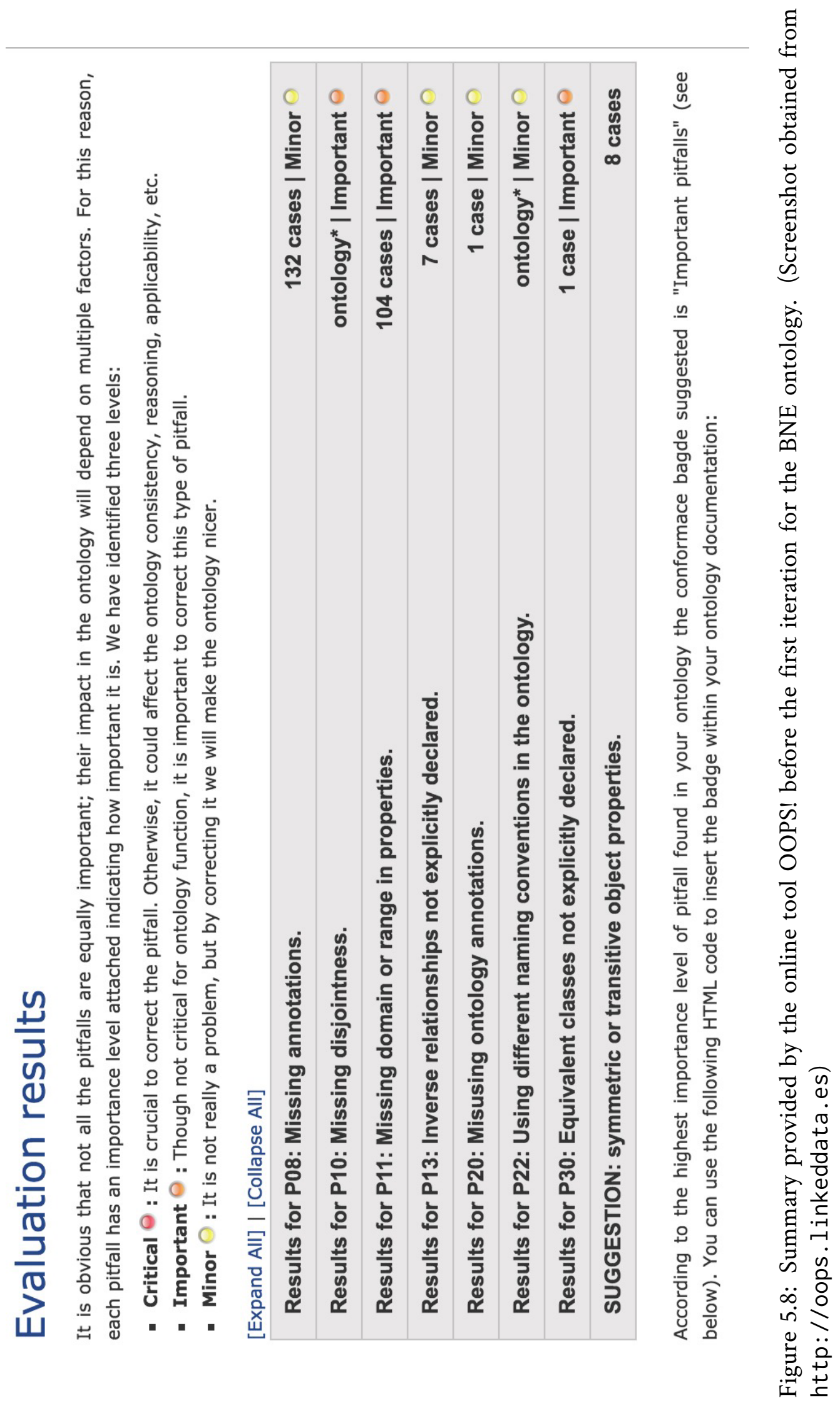


to existing standards such as FRBR and ISBD, which already provide precise descriptions.

- P10: Missing disjointness (Important). This pitfall indicated that none of the six classes of the BNE ontology were defined as disjoint of one another. As this was an important pitfall, the ontology engineers added several owl : disjointWith statements to represent that every class in the BNE ontology is disjoint with the rest of the classes.

- P11: Missing domain or range in properties (Important). This pitfall indicated the lack of a large number of domain and ranges declarations for properties. As explained in Section 5.1.2, the mapping templates include a column to define the RDFS domain of datatype properties and object properties (see Figure 5.2). These domain and range declarations were added to the BNE ontology during the ontology implementation activity of phase 3 . However, the majority of missing domains indicated by OOPS! corresponded with missing ranges for datatype properties, which are not addressed by the mapping templates. After analyzing the missing domains and ranges, the ontology development team did not consider this pitfall as critical for the publication of the ontology.

- P13: Inverse relationships not explicitly declared (Minor). This pitfall indicated the lack of seven owl: inverse0f statements for object properties. After analyzing the missing statements, the ontology development postponed their inclusion until the next iteration.

- P20: Misusing ontology annotations (Minor). This pitfall indicated one case of potentially incorrect use of an annotation for a datatype property. In this case, the tool was really useful as it helped to detect a problem that occurred during the ontology localization activity, which was a highly manual activity. In particular, instead of adding an extra rdfs: label with a language tag, the ontological engineers had incorrectly added rdfs: comment. This pitfall was easily repaired during this iteration.

- P22: Using different naming conventions in the ontology (Minor). This pitfall indicated a potential inconsistency in the BNE ontology naming policy, which has been presented in Section 5.5. Unfortunately, the tool did not provide any further guidance that made it possible to fix this issue. 
- P30: Equivalent classes not explicitly declared (Important). This pitfall stated that the class Expression (bne:C1002) and the class Work (bne:C1001) were equivalent. However, no further explanation was provided, and, after careful evaluation, the ontology engineers did not find any statement producing the inconsistency highlighted by the tool.

Second iteration. This iteration was carried out after the publication of the first version of the BNE ontology, before a new release of the datos.bne.es service. The tool detected the five remaining pitfalls from the first iteration (two pitfalls labelled as important and three as minor). The second iteration focused on repairing one of the two pitfalls labelled as important (P11). In fact, after contacting the authors of the OOPS! tool, the remaining important pitfall was found to be not applicable in this case. Moreover, the ontology development team tackled one of the minor pitfalls (P13), as we describe below:

- P11: Missing domain or range in properties (Important). It is a known issue that MARC 21 metadata elements do lack a standard structure for most of the fields (e.g., fields related to dates usually contain textual descriptions, not well-formatted dates). Therefore, the safer range for datatype properties in this context is rdfs:Literal, which were added to the datatype properties declarations in this iteration. Moreover, some missing ranges for object properties were detected, which were included in this iteration by the ontology engineers.

- P13: Inverse relationships not explicitly declared (Minor). During this iteration, the ontology development team was able to repair four out of seven owl : inver se0f statements for object properties. The remaining owl: inverse0f statements were actually not correctly identified by OOPS! and corresponded to object properties that had been purposedly declared without inverse properties in the BNE ontology.

\subsection{Summary}

In this chapter we have described our methodological and technological contribution for building ontology networks to model library data. These methodological aspects are integrated into our ontology-based data framework and build on the methods described along the thesis. In particular, we have proposed an ontology network life- 
cycle model that enables the participation of domain experts during the whole lifecycle and specially during the ontology design process. Moreover, we have introduced a new activity Ontology publication, that facilitates the potential reuse and the understanding of the ontology network. This contribution has focused on our third hypothesis $\mathrm{H} 3$ that is formulated as follows: Systematic approaches based on catalogue data and domain expert inputs can produce an ontology with sufficient quality. To study this hypothesis, we have described the application of the proposed life-cycle model for building an ontology network to model the National Library of Spain catalogue. More importantly, the resulting ontology network has been evaluated with a topology-based approach. Specifically: $i$ ) no critical issues were found in the BNE ontology, indicating the sufficient quality of the ontology; $i i)$ seven pitfalls were identified during the first iteration, three of them labelled as important; and, iii) during the second iteration, the important pitfalls were repaired, leaving only two minor pitfalls that did not critically affect the quality of the BNE ontology.

The BNE ontology network is the backbone of the datos.bne.es data set and service. This data set and service will be introduced in the next chapter and then used in Chapter 8 for the evaluation of our last hypothesis H5, regarding the impact on enduser applications, which adds an application-based evaluation of the BNE ontology (see definition in Section 5.6.2). 


\section{Topic-based ontology similarity}

As discussed in Chapter 2, there are many available and overlapping library ontologies, which makes it difficult for ontological engineers and domain experts to compare them. Moreover, as reviewed in Section 2.4.5, the majority of approaches for ontology search and similarity are either based on structural properties of the ontologies or on string similarity measures. In this thesis, we hypothesize that probabilistic topic models can be used to provide improved capabilities to compare overlapping ontologies in the library domain.

To this end, the contribution of this chapter is focused on our second research problem (P2) and in particular on the research question: Can we develop methods that help understanding thematic relations between library ontologies?. This contribution is used in this chapter to evaluate our fourth hypothesis, which states that: probabilistic topic modelling techniques can produce coherent descriptions of ontologies and perform better in terms of precision than classical term-count based methods for ontology search.

The rest of this chapter is organized as follows. First, we provide an introduction to topic models and topic modelling techniques. Second, we provide the set of formal definitions and the notation that will be used to describe our contribution. Third, we describe our proposed method, marimba-topicmodel, and illustrate its with a real example from the library domain. Finally, we describe the set of experiments carried out using marimba-topicmodel to validate our fourth hypothesis and discuss the results of the evaluation. 


\subsection{Introduction to topic models}

In the literature, the terms topic modelling and topic model are frequently used interchangeably to refer to the algorithms, the task, and the probabilistic model. Here we will refer to topic model as the probabilistic model, and topic modelling as the task and methods to build a probabilistic model of a corpus. Probabilistic topic modelling algorithms are statistical methods that analyze the words in large corpora of documents to discover the thematic structure of documents. Topic modelling algorithms can be thought of as unsupervised machine learning algorithms, in the sense that they do not require any prior annotations of the analyzed documents for learning the thematic structure.

Topic models are generative probabilistic models. In generative probabilistic models, the data is observed as being generated by a generative process that includes hidden or latent random variables. This generative process defines a joint probability distribution over the observed and latent random variables. The main objective of topic modelling techniques is to compute the conditional distribution of the latent variables given the observed variables. To introduce topic models, we will use Latent Dirichlet Allocation model (LDA) by Blei et al. [2003], which is the most cited and widely-used topic model. Most of the other topic models in the literature are variations or extensions of the ideas behind LDA.

LDA formally defines a topic as a probability distribution over the fixed vocabulary of a document corpus. For example the artificial intelligence topic has words related to natural language processing with high probability, and words about french literature with lower probability. LDA assumes a generative process where the set of topics has been generated before any other data in the corpus. Once the set of topics has been generated, the documents are generated in two steps. Specifically, for each document in the corpus:

1. A distribution over topics for the document is randomly chosen. Loosely speaking, this distribution corresponds to the proportions of each topic in the document. This is known as the per-document topic distribution. For example, an article about natural language processing will have $60 \%$ of the words about artificial intelligence, $35 \%$ of the words about linguistics, and even $5 \%$ of the words about literature if the article deals with natural language processing applied to literary texts. 
2. Then, for each word in the document

a) A topic is randomly chosen from the per-document topic distribution of step 1. Continuing with our example, for the natural language processing article, the artificial intelligence topic will have higher probability of being chosen in this step.

b) A word is randomly chosen from the distribution over the vocabulary corresponding to the topic of step 2a. For our article and having chosen the artificial intelligence topic, words highly related to the artificial intelligence topic will have higher probability of being chosen, and words barely related to the topic will have lower probability.

In the above generative process, the observed variables are the words in the documents of the corpus, and the latent variables are the distribution over the vocabulary for each topic, and the per-document topic distributions. In this way, the computational problem tackled by LDA is to infer the latent variables that most likely generated the observed corpus. In formal terms, this problem corresponds to computing the conditional distribution of the latent variables given the documents in the corpus. This conditional distribution is also called the posterior distribution. Unfortunately, the computation of the posterior distribution is intractable because it would mean to compute every possible instantiation of the latent topic structure for the observed words in the corpus. For this reason, as with other probabilistic models, the goal of topic modelling algorithms is to approximate the posterior distribution. Loosely speaking, topic modelling algorithms infer a distribution over the latent variables that is close to the true posterior distribution. Regarding the approximation or training methods, topic modelling algorithms typically fall into two distinct categories:

1. Sampling methods, which attempt to collect samples from the posterior to approximate it with an empirical distribution. A notable example of this category is the collapsed Gibbs sampling method (Griffiths and Steyvers [2004]).

2. Variational methods, which are deterministic methods that posit a parameterized family of distributions over the hidden structure and then find the member of that family that is closest to the posterior distribution. A survey of methods belonging to this category is provided in Jordan et al. [1999]. 
Each category of methods presents drawbacks and advantages. ${ }^{1}$ An in-depth comparison of different training methods is out of the scope of this thesis. In our experimental evaluation, we will use the collapsed Gibbs sampling method (Griffiths and Steyvers [2004]), but it is worth noting that marimba-topicmodel can be trained with any other sampling or variational method.

An important characteristic in order to measure the effectiveness of topic models is the length of the documents to be modelled. In this sense, LDA and its extensions (e.g., Hoffman et al. [2010]) have proven to be an effective and scalable solution to model the latent topics of collections of relatively large documents. However, LDA has been shown to suffer from the data sparsity of words within short documents. In this thesis, we hypothesis that documents extracted from ontological descriptions are frequently short and LDA can suffer from data sparsity issues. To alleviate the data sparsity issues on short text, several approaches have been proposed. These approaches can be classified into two categories:

1. Extensions to LDA, which use aggregated or external information before training an LDA topic model to mitigate the data sparsity problem. Approaches of this category have the limitation of depending on the availability of external information in order to train the topic model (Weng et al. [2010]; Hong and Davison [2010]).

2. New topic models, which propose a new statistical topic model. These approaches provide modifications to the topic model to mitigate the data sparsity problem and work directly with the documents without any additional source of information (Zhao et al. [2011], Nigam et al. [2000])

The most notable approach focused on short-text documents is the Biterm Topic Model (BTM) proposed by Cheng et al. [2014], which belongs to the second category. BTM that has been shown to perform better than LDA and other approaches for shorttext documents (Zhao et al. [2011], Nigam et al. [2000]).

\subsection{Contributions}

Given the effectiveness of topic models in revealing the latent structure of large collections of documents and the growing number of ontologies on the Web, in this the-

\footnotetext{
${ }^{1}$ For an in-depth discussion on sampling and variational methods, we refer the reader to the article by Asuncion et al. [2009].
} 
sis we propose the marimba-topicmodel method. marimba-topicmodel is a novel method that applies word-sense disambiguation and topic modelling to ontologies to reveal their latent thematic structure. The extraction of the ontology latent topics enables the comparison of ontologies in terms of thematic relatedness, providing sophisticated mechanisms to identify similarities among ontologies. The extracted latent topics can be used for enhancing applications such as ontology clustering, ontology search, and ontology matching.

As we will detail in this section, marimba-topicmodel applies word-sense disambiguation before training the topic model, with the goal of $i$ ) reducing lexical ambiguity thus improving the coherence of topics, and $i i$ ) linking the topic terms to the Linked Open Data (LOD) cloud, opening up new possibilities for the exploitation of topics.

In order to infer the latent thematic structure of a corpus of ontologies, marimbatopicmodel performs three main steps:

1. Extraction of ontology documents from a corpus of ontologies. This step extracts the textual descriptions of the ontologies in the corpus and creates an ontology document for each ontology using the words in their textual descriptions.

2. Annotation of the words in the ontology documents with external senses using word-sense disambiguation. This step processes the ontology documents and annotates their words with senses using word-sense disambiguation. We hypothesize in this thesis that by disambiguating the words in the ontology documents, we can increase the coherence of the produced topics.

3. Training of a topic model using the sense-annotated ontology documents. This step uses the sense-annotated ontology documents to train a topic model. In this step, the main decision is which topic model to use. We hypothesize that sense-annotated ontology documents will suffer from data sparsity problems due to their short length and noisy nature.

Based on the above topic model, in this contribution we introduce two topic-based ontology similarity measures. The remainder of the chapter is organized as follows. In Section 6.3 we introduce the notation and main concepts used in the chapter. In Section 6.4, we describe in detail the marimba-topicmodel method and introduce a real application to a corpus of library ontologies. Finally, in Section 6.5 we describe and discuss two experiments to validate our main hypothesis. 


\subsection{Notation and definitions}

In this chapter we use classical terminology for describing text collections and refer to entities such as "words", "documents", and "corpora". In particular, an ontology can be defined as a "document" containing a collection of "words" that describe the entities and properties of the domain covered by the ontology. A collection of ontologies can be then defined as a "corpus" of documents. The rationale of this terminology is providing an intuitive framework for describing the different techniques in a way that is easy to understand and that is aligned with classical information retrieval terminology.

For dealing with words, documents and corpora, we use the following definitions adapted from Blei et al. [2003]:

Definition 6.3.1 (Word) A word is our basic unit of discrete data. A word is an item from a vocabulary indexed by $\{1, \ldots, V\} . V$ is the size of the vocabulary containing every word in a certain collection. Words are represented as vectors of size $V$ that have a single component equal to one and all other components equal to zero. We use superscripts to denote the vector components and the vth word in the vocabulary is denoted by a vector $w$ where $w^{v}=1$ and $w^{u}=0$ for every $u \neq v$.

Definition 6.3.2 (Document) A document is a sequence of $n$ words denoted by $\mathbf{d}=\left(w_{1}, w_{2}, \ldots, w_{n}\right)$, where $w_{i}$ is the $i$ th word in the sequence. In our case, an ontology is represented as a document where the sequence of words corresponds to the words used to describe the different ontological entities ${ }^{2}$ (see definition of ontology document below).

Definition 6.3.3 (Corpus) A corpus is a collection of $m$ documents denoted by $\mathbf{D}=\left\{d_{1}, d_{2}, \ldots, d_{m}\right\}$.

\subsubsection{Ontology documents}

In order to align these definitions with the terminology of the ontology field, we use the following definitions:

Definition 6.3.4 (Ontology) An ontology is a collection of $p$ ontology entities denoted by $\mathbf{O}=\left\{e_{1}, e_{2}, \ldots, e_{p}\right\}$.

\footnotetext{
${ }^{2}$ In our context, ontology entities refer to the classes, properties and individuals of the ontology.
} 
Definition 6.3.5 (Ontology document) An ontology document is a sequence of $n$ words denoted by $\mathbf{d}=\left(w_{1}, w_{2}, \ldots, w_{n}\right)$, where $w_{i}$ is the ith word in the sequence and the words have been extracted from the textual descriptions of ontology entities within an ontology $O$.

\subsubsection{Topic models}

To introduce the notation for defining topic models, in Figure 6.1 we present the LDA model using the standard notation for graphical models. This notation reflects, in an intuitive way, the conditional dependencies between the observed and the latent random variables. In the figure, each node is a random variable. The latent variables are unshaded and correspond to the topic proportions for each document $\theta_{d}$, the topic assignments for each word $Z_{d, n}$, and the topic distributions $\varphi_{k}$. The observed variables are shaded and correspond to the words of each document $W_{d, n}$. LDA typically uses symmetric Dirichlet priors for initializing $\theta_{d}$ and $\varphi_{k}$. These Dirichlet priors are parameterized using the hyperparameters ${ }^{3} \alpha$ and $\beta$. The rectangles are in plate notation (Buntine [1994]) and denote replication. We provide the complete definitions below:

Definition 6.3.6 (Words $W_{d, n}$ ) The words $W_{d, n}$ are the observed random variable of the model and correspond to the sequence of words of each document $d \in D$.

Definition 6.3.7 (Per-document topic distribution $\theta_{d}$ ) The per-document topic distribution $\theta_{d}$ is the topic distribution for document $d$.

Definition 6.3.8 (Per-topic word distribution $\varphi_{k}$ ) The per-topic word distribution $\varphi_{k}$ is the word distribution for topick.

Definition 6.3.9 (Per-word topic assignment $Z_{d, n}$ ) The per-word topic assignment $Z_{d, n}$ is the topic assigned to the $n$th word in document $d$.

Definition 6.3.10 $(\alpha)$ The hyperparameter $\alpha$ is the parameter of the Dirichlet prior on the per-document distributions.

Definition 6.3.11 $(\beta)$ The hyperparameter $\beta$ is the parameter of the Dirichlet prior on the per-topic word distributions.

\footnotetext{
${ }^{3}$ Hyperparameter is the common term for defining initialization parameters configured by the users of statistical models.
} 


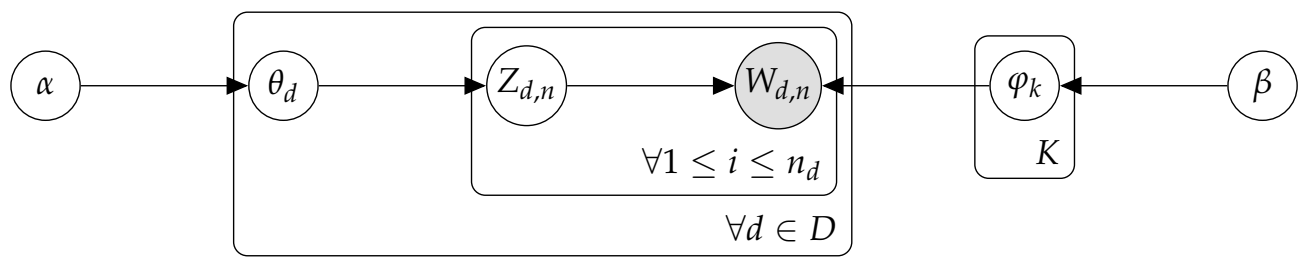

Figure 6.1: Graphical model representation of the conditional dependencies of LDA. Each node is a random variable. The latent variables are unshaded and correspond to the topic proportions $\theta_{d}$, the topic assignments for each word $Z_{d, n}$, and the topic distributions $\phi_{k}$. The observed variables are shaded and correspond to the words of each document $W_{d, n}$. The variable $\alpha$ determines the topic distribution for each document. The rectangles are in plate notation and denote replication (Adapted from Blei [2012])

Besides LDA, in this thesis we will use the Biterm Topic Model (BTM). The complete description of BTM can be found in Cheng et al. [2014]. BTM directly models word co-ocurrence patterns within the whole corpus of documents, thus making the word co-ocurrence frequencies more stable and eventually mitigating data sparsity issues in short-text documents. Specifically, instead of modelling the document generation process like in LDA, BTM models the co-ocurrence of words using biterms. We define a biterm as follows:

Definition 6.3.12 (Biterm) A bitermb is an unordered combination of every two words in a documentd. For example, given a document d with three words such as (author, name, work), the biterm set $b$ generated for d corresponds to \{(author,name),(author,work),(work,name)\}.

Definition 6.3.13 (Corpus biterm set) A corpus biterm set $B$ is extracted for a corpus of documents $D$ by combining the biterms of each document.

BTM uses the corpus biterm set $B$ to carry out the inference of the model parameters using sampling methods, such as the collapsed Gibbs sampling method (Griffiths and Steyvers [2004]), which will be used in the evaluation of the marimba-topicmodel method. As shown in Figure 6.2, the main differences with respect to LDA are: $i$ ) BTM uses a global topic distribution $\theta$ instead of per-document topic distributions $\theta_{d}$; and $\left.i i\right)$ the observed random variables are the words $W_{i}$ and $W_{j}$ of each biterm in the corpus. As mentioned above, BTM does not directly model the per-document topic distribution $\theta_{d}$ during the inference process. Instead, the topic proportion of a document $\theta_{d}$ can be derived using the topics of biterms. ${ }^{4}$

\footnotetext{
${ }^{4}$ The complete details on this derivation can be found in Cheng et al. [2014].
} 


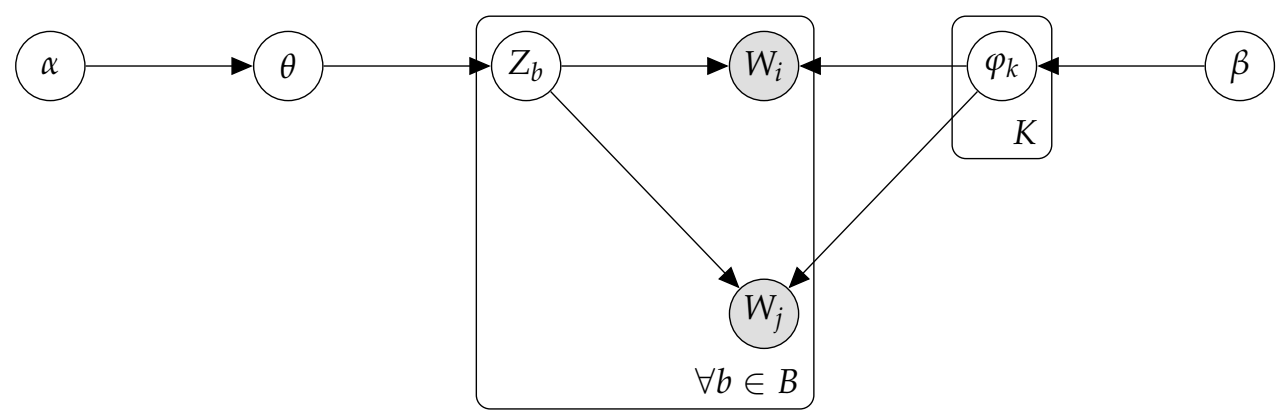

Figure 6.2: Graphical model representation of the conditional dependencies of BTM. Each node is a random variable. The latent variables are unshaded and correspond to the global topic proportions $\theta$, the topic assignments for each biterm $Z_{b}$, and the topic distributions $\phi_{k}$. The observed variables are shaded and correspond to each pair of words in the corpus corpus biterm set $B, W_{i}$ and $W_{j}$. The variable $\alpha$ parameterizes the global topic distribution. The rectangles are in plate notation and denote replication (Adapted from Cheng et al. [2014])

\subsubsection{Topic-based ontology similarity}

As described above, probabilistic topic models can represent ontology documents as a probability distribution over topics or topic proportions (i.e., the per-document topic distribution $\theta_{d}$ ). Specifically, through the inference process, each ontology document is represented as a vector of size $\mathrm{K}$ (i.e., the number of topics used for the model inference) and each position $k$ in the vector contains the probability of that document having the $k t h$ topic. Formally, a document is represented as follows:

$$
d_{i}=\left[p\left(z_{1} \mid d_{i}\right), \ldots, p\left(z_{k} \mid d_{i}\right)\right]
$$

where $z_{k}$ is the topic assignment for the $k t h$ topic.

Given such probability distributions for each ontology document, we can measure the similarity between two ontology documents $d_{i}$ and $d_{j}$ using the widely-used Jensen-Shannon divergence (JSD) (Lin [1991]), which is an information-theoretic measure to compare probability distributions. Furthermore, the Jensen-Shannon divergence measure has been extensively used for document classification tasks involving topic distributions (e.g., Cheng et al. [2014]). Formally, the Jensen-Shannon divergence of two probability distributions is defined as follows:

$$
J S D\left(d_{i}, d_{j}\right)=\frac{1}{2} D_{K L}\left(d_{i}|| R\right)+\frac{1}{2} D_{K L}\left(d_{j}|| R\right)
$$


where $R=\frac{d_{i}+d_{j}}{2}$ is the mid-point measure and $D_{K L}(\cdot \| \cdot)$ is the Kullback-Leibler divergence (Lin [1991]), which is defined as:

$$
D_{K L}(p \| q)=\sum_{i} \log \left(\frac{p_{i}}{q_{i}}\right) p_{i}
$$

The Jensen-Shannon divergence is a symmetric and bounded measure, which provides values in the $[0,1]$ range. The intuition is that smaller values of JSD mean that two ontology documents are topically similar. This intuition can be used as a distance measure, although $J S D$ is not a formal distance metric. Given its effectiveness and simplicity for measuring the similarity of topic probability distributions, we will use Jensen-Shannon divergence measure as our main measure for topic-based ontology similarity. Moreover, the square root of the JSD measure, frequently known as Jensen-Shannon distance or Jensen-Shannon metric (JSM) has been shown to be a distance metric in Endres and Schindelin [2003]. Formally, the Jensen-Shannon metric is defined as follows:

$$
\operatorname{JSM}\left(d_{i}, d_{j}\right)=\sqrt{\operatorname{JSD}\left(d_{i}, d_{j}\right)}
$$

\subsection{Marimba Topic Model}

The input to marimba-topicmodel is a corpus $O$ of ontologies and the output is a probabilistic topic model $T$. The marimba-topicmodel method performs three steps: i) extraction of the ontology documents from the textual descriptions contained in the ontologies, $\mathrm{i}$ ) annotation of the words in the ontology documents with external senses using word-sense disambiguation, $\mathrm{iii}$ ) modelling ontology topics based on the annotated words using a probabilistic topic model. Algorithm 2 provides an overview of the inputs, outputs and three main steps of marimba-topicmodel. We describe each of the steps in the following sections.

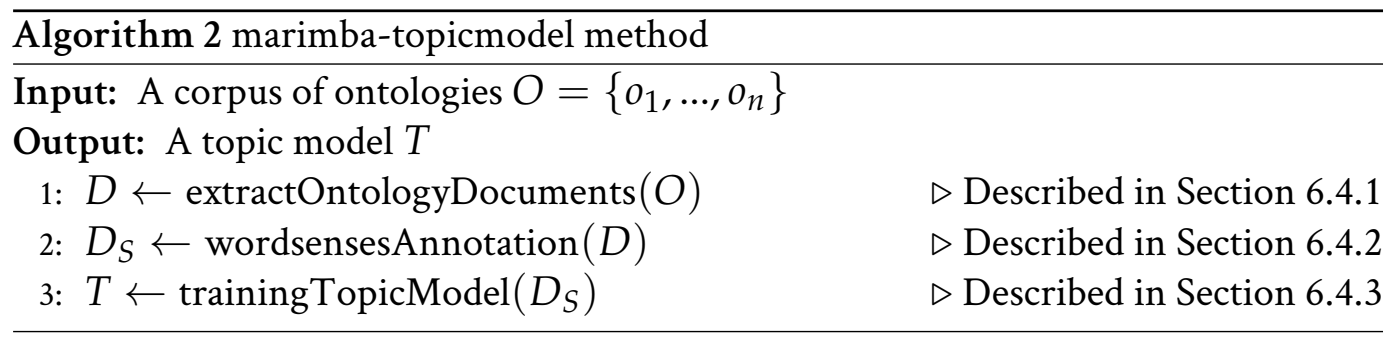


To illustrate the marimba-topicmodel method in this section, we will use Bibo and $\mathrm{FaBiO}^{5}$, two well known library ontologies. In this section, we show only partial samples of the results, the complete results can be found in the additional online material. ${ }^{6}$

\subsubsection{Ontology document extraction}

In the ontology document extraction step, our method extracts one ontology document for each ontology in the input corpus $O$. The goal of the step is to extract the textual description of each ontology element in the ontology.

A variety of extraction functions can be built to extract the textual descriptions of an ontology element. In our case, we extend the implementation developed in CIDERCL by Gracia and Asooja [2013], a tool for ontology alignment and semantic similarity computation. In our method, we limit the extracted ontological elements to the IRI local name and every property value whose data type is a literal. ${ }^{7}$ In this way, each ontology document is constructed as text directly extracted and concatenated from the textual descriptions of each ontology element. This representation is one of the main assumptions of LDA and the topic models used in this thesis. The algorithm for the ontology document extraction step is presented below in Algorithm 3.

The application of the ontology document extraction step to the ontologies $O_{B i b o}$ and $o_{F a B i O}$ produces the following ontology documents:

$$
\begin{gathered}
d_{\text {Bibo }}=(\text { author }, \text { workshop }, \text { chapter }, \text { edition }, . . .) \\
d_{F a B i O}=(\text { edition, preprint, created, workshop }, . . .)
\end{gathered}
$$

\subsubsection{Word-senses annotation}

In the second step, the input is the corpus of ontology documents $D$ generated by the ontology document extraction step and the output is the corpus $D_{S}$ of sense-annotated ontology documents. The goal of the word-senses annotation step is to annotate each ontology document of $D$ with senses using a word-sense disambiguation (WSD) method. Word sense disambiguation deals with reducing lexical ambiguity by linking singleword and multiword units to their meanings, also known as senses, based on their

\footnotetext{
${ }^{5}$ http://www.essepuntato.it/lode/http://purl.org/spar/fabio (Last viewed 13th May 2016)

${ }^{6}$ https://github.com/dvsrepo/tovo

${ }^{7} \mathrm{~A}$ literal in ontology terminology is a string.
} 


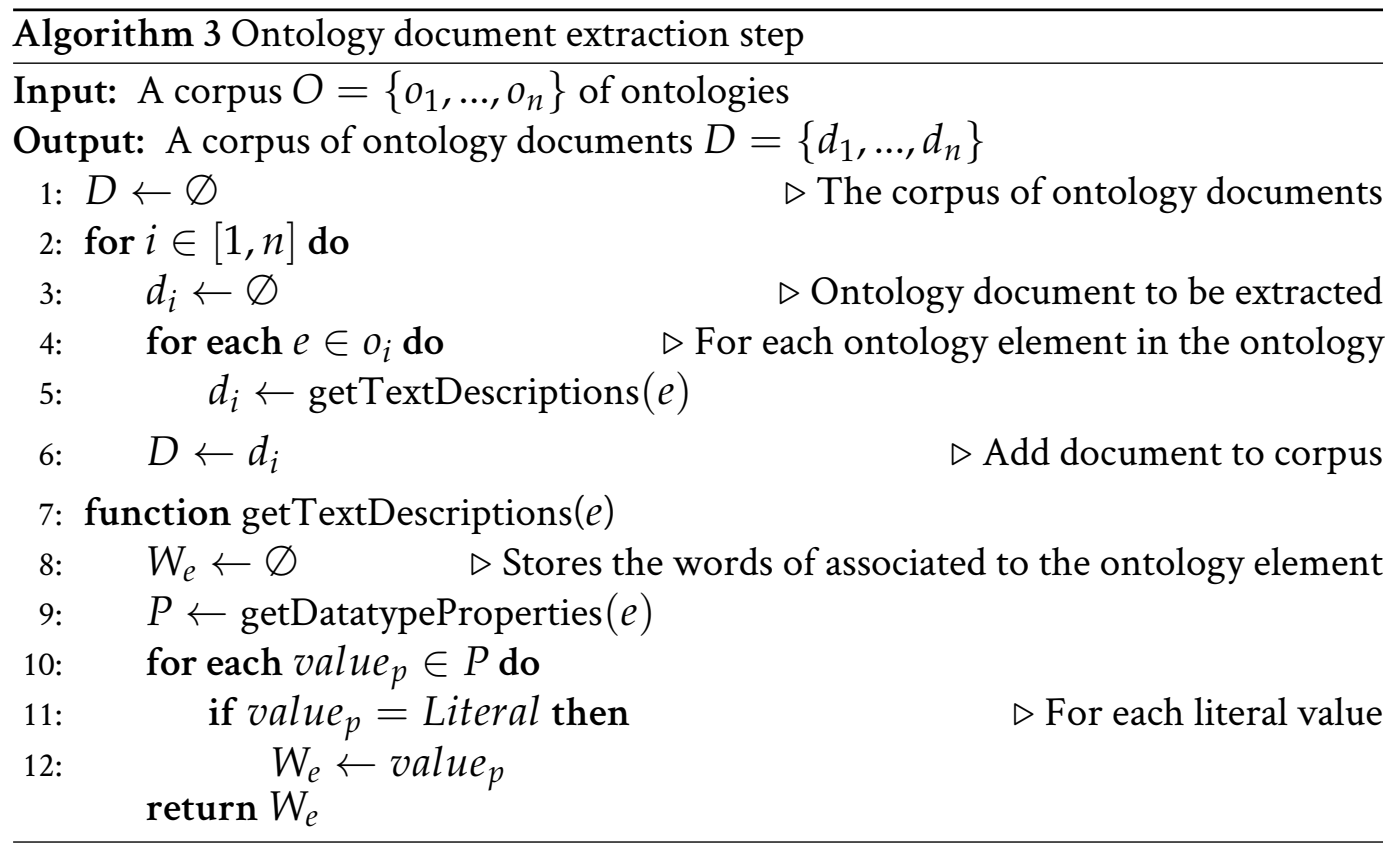

context (Navigli [2009]). Our underlying hypothesis is that by disambiguating the words in the ontology documents and by linking them to external senses, their lexical ambiguity is reduced and thus the coherence of the generated topics is expected to be higher.

Although the marimba-topicmodel method could operate with any WSD tool, we evaluate our hypothesis using the tool Babelfy ${ }^{8}$ (Moro et al. [2014]). The Babelfy tool has shown state-of-the-art performance and presents the additional benefit of linking senses to a large multilingual knowledge base, BabelNet (Navigli and Ponzetto [2012]), which integrates several wordnets, Wikidata, Wikipedia, and other large web resources. Additionally, Babelfy presents the following desirable properties within our context:

1. Babelfy can identify senses of words and multi-words in sequences of words with maximum length of five, which contain at least a noun. Ontology contexts extracted from classes, properties, and individuals show a significant predominance of (compound) nouns, and usually do not conform entire sentences, for instance Person, Corporate Body, or name of person.

2. Babelfy is a graph-based approach that relies exclusively on similarity of semantic signatures derived from the Babelnet graph, taking individual candidates

\footnotetext{
${ }^{8}$ The tool is described at http: //babelfy.org/about (Last viewed 25th April 2016)
} 
from the text and not relying on the sentence structure, which can mitigate the lack of structure of ontology contexts.

Using Babelfy, the ouput of this step is a corpus $D_{S}$ where each $d \in D$ is annotated with sense IDs coming from BabelNet (in BabelNet, senses are called BabelSynsets). A sense ID is an IRI within the http://babelnet.org/rdf/ namespace. For example, $b n: 00046516 n$ is one of the sense IDs corresponding to the word "person".

The Babelfy tool provides a web API to annotate the documents with sense IDs. As shown in Algorithm 4, for each ontology document $d \in D$, the word-senses annotation method calls the Babelfy API (Line 5). The Babelfy API call retrieves the corresponding sense IDs, which are then used to build the sense-annotated document added to $D_{S}$ (Line 6). It is worth noting that sense-annotated ontology documents contain exclusively sense IDs and the word-senses annotation method removes the words that could not be annotated by Babelfy.

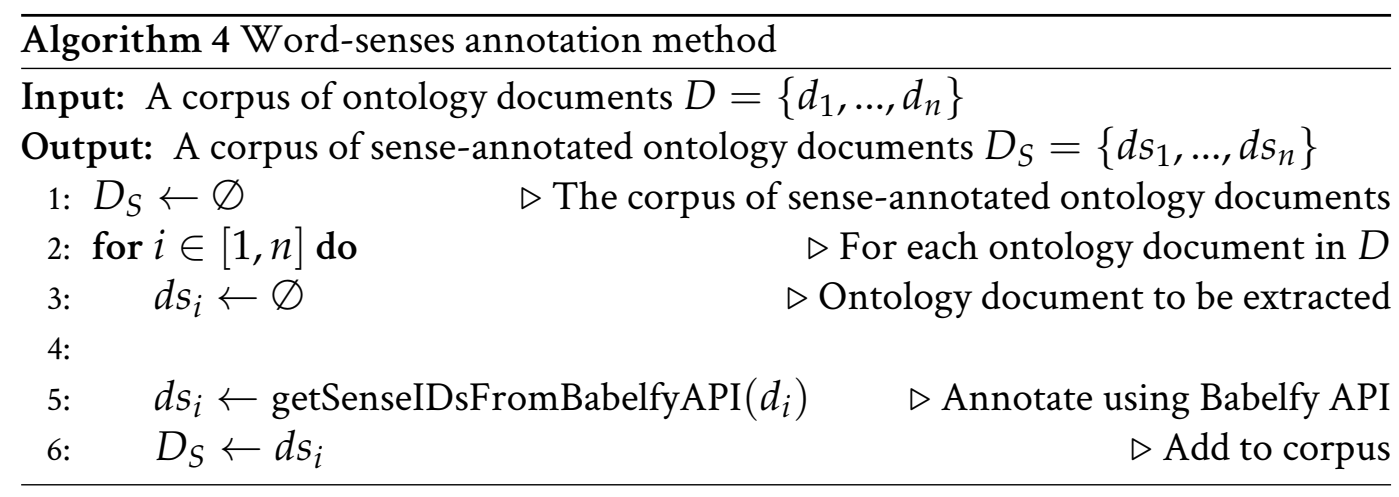

For example, given an ontology document $d$ containing five words (info, data, email, person, nomen), the word-senses annotation step will create senseannotated ontology document with four BabelNet sense IDs (bn:s00046705n,bn:s00025314n,bn:00029345n,bn:00046516n). As it can be seen from the example, the word "nomen" will be excluded as it is not annotated with a sense ID. As a more complete example, the application of the word-sense annotation step to the ontology documents $d_{B i b o}$ and $d_{F a B i O}$ produces the results shown in Table 6.1.

\subsubsection{Topic modelling}

The final step consists in using the sense-annotated ontology documents of $D_{S}$ to train a topic model. The inputs to this step are the topic model hyperparameters $\alpha$ and $\beta$, 
Table 6.1: Excerpt of Bibo and $\mathrm{FaBiO}$ ontology documents annotated with the wordsenses annotation method. The prefix bn: corresponds to http://babelnet.org/rdf/

\begin{tabular}{lcccc}
\hline Ontology & Senses (terms) & & & \\
\hline Bibo & bn:s00007287n & bn:s00071216n & bn:s00182115n & bn:s00029770n \\
& (author) & (workshop) & (chapter) & (edition) \\
FaBiO & bn:s00029770n & bn:s00823736n & bn:s00086008v & bn:s00071216n \\
& (edition) & (preprint) & (created) & (workshop) \\
\hline
\end{tabular}

the number of topics $K$, the number of iterations of the sampling method, and the corpus of sense-annotated ontology documents $D_{S}$. Regarding the topic model hyperparameters and the number of topics, we provide a discussion of different settings in the evaluation section (Section 6.5). The output of this step are the inferred parameters after training the topic model: $i)$ the per-document topic distribution $\left(\theta_{d}\right)$; and, ii) the per-topic word distribution $\left(\phi_{k}\right)$.

As discussed in Section 6.2, we hypothesize that BTM will outperform LDA for modelling topics within ontology documents. Therefore, in this section we describe the procedure followed by marimba-topicmodel to train a BTM topic model using sense-annotated ontology documents. We use the collapsed Gibbs sampling method (Griffiths and Steyvers [2004]) for inferring the model parameters. The collapsed Gibbs sampling method estimates the model parameters using samples drawn from the posterior distributions of the latent variables sequentially conditioned on the current values of all other variables and the observed data.

The method is presented in Algorithm 5. First, the corpus biterm set $B$ is generated from the corpus of sense-annotated ontology documents $D_{S}$ (Line 1). Second, a topic is randomly assigned to each biterm in the corpus biterm set (Line 2). Then, the sampling method is executed during the number of iterations defined by $N_{i t e r}$ (Line 3). In each iteration, the topic assignment for each biterm $b_{i}$ in $B$ is updated sequentially by calculating the conditional probability ${ }^{9} \mathrm{P}\left(z_{i} \mid z_{-i}, B\right)$, where $z_{-i}$ denotes the topic assignments for all biterms except $b_{i}$ (Line 5). Then, the number of biterms $n_{k}$ in each topic $k$, and the number of times each word is assigned to topic $k$, denoted by $n_{w_{i, 1} \mid k}$ and $n_{w_{i, 2} \mid k}$, are updated (Line 6). Finally, the counts $n_{k}$ and $n_{w \mid k}$ are used to estimate

\footnotetext{
${ }^{9}$ The complete details about the derivation of the conditional probability distribution can be found in Cheng et al. [2014] and http: //doi . i eeecomputersociety.org/10.1109/TKDE. 2014. 231387 (Last viewed 26th April 2016)
} 
$\phi$ and $\boldsymbol{\theta}$ as follows:

$$
\phi_{k, w}=\frac{n_{w \mid k}+\beta}{n_{. \mid k}+W \beta}
$$

where $n_{. \mid k}$ is the total number of biterms assigned to topic $k$ and $W$ is the total number of words in the corpus.

$$
\theta_{k}=\frac{n_{k}+\alpha}{N B+K \alpha}
$$

where $N_{B}$ is the total number of biterms in the corpus biterm set.

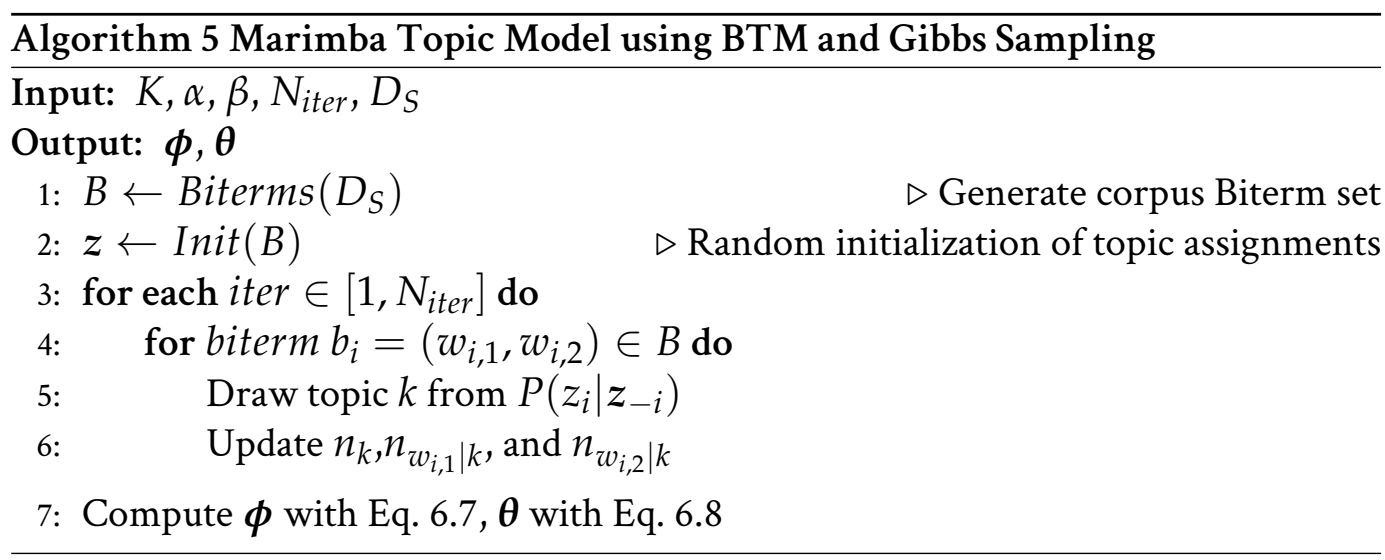

For example, if we apply the topic modelling method to a corpus of library ontologies $D_{S}$ with twenty topics $(K=20)$, we observe that the per-document topic distribution for Bibo and $\mathrm{FaBiO}$ are very similar. In particular, both present a high probability for topic $k=8$, meaning that this topic is a highly descriptive topic for both ontologies. Also, they present higher probability for other topics (fourth and eighteenth), indicating other thematic aspects of the ontologies. We show below the distribution for the three most probable topics:

$$
\begin{aligned}
& d_{\text {Bibo }}=\left[. .,(0.1242)_{4}, . .,(\mathbf{0 . 6 7 0 7})_{8}, . . .,(0.0899)_{18}, . .\right] \\
& d_{\mathrm{FaBiO}}=\left[. .,(0.0397)_{4}, . .,(0.0308)_{6}, . .,(\mathbf{0 . 8 4 4 9})_{8}, . .\right]
\end{aligned}
$$

Regarding the per-topic word distribution, the topic $k=8$ can be represented by ranking its most probable words. We show below the nine most probable words and their corresponding senses for topic $k=8$, which is the most probable topic for Bibo and $\mathrm{FaBiO}$ : 


$$
\begin{gathered}
\text { bn:s00046705n (info) } \\
\text { bn:s00025314n (data) } \\
\text { bn:s00029345n (email) } \\
\text { bn:s00046516n (person) } \\
\text { bn:s00049910n (language) } \\
\text { bn:s00023236n (country,nation) } \\
\text { bn:s00021547n (concept) } \\
\text { bn:s00047172n (site,website) } \\
\text { bn:s00052671n (journal,periodical,magazine) }
\end{gathered}
$$

\subsubsection{An application of Marimba Topic Model}

We conclude this section by discussing a direct application of marimba-topicmodel to a set of library-related ontologies. We selected twenty-three ontologies from the LOV corpus, including specialized library ontologies such as IFLA ISBD, the RDA family of vocabularies, or BIBFRAME, as well as more general vocabularies such as schema.org, the DBpedia ontology, and the FOAF ontology ${ }^{10}$.

In this example, we performed the following steps:

1. We applied marimba-topicmodel with $K=20$ to the LOV corpus.

2. After applying our method, we obtained the per-document topic distribution for each ontology, and used these distributions to analyze their similarity. We used the topic-based ontology similarity based on JSD, which we defined in Section 6.3.

3. We applied the JSD measure to each pair of the selected library ontologies and obtain a $23 \times 23$ divergence matrix. Using this matrix, for each vocabulary we obtained the most topically-similar ontologies.

For instance, the ontologies most similar to $\mathrm{FaBiO}^{11}$ are $\mathrm{Bibo}(0.06)$, RDA properties for describing manifestations (0.07), and FOAF (0.09). Bibo is the most similar ontology because it models very similar entities, especially those related to academic publishing. Regarding RDA properties for manifestations, it is a FRBR-based vocabulary composed exclusively by properties to model bibliographic entities and that can in fact be used in combination with $\mathrm{FaBiO}$ which is also FRBR-based. Finally, FOAF and $\mathrm{FaBiO}$ are closely related in the way they model personal and biographical information.

\footnotetext{
${ }^{10}$ The complete list is shown on the right-hand side of Figure 6.3.

${ }^{11}$ The ontologies are ordered by ascending values of JSD.
} 


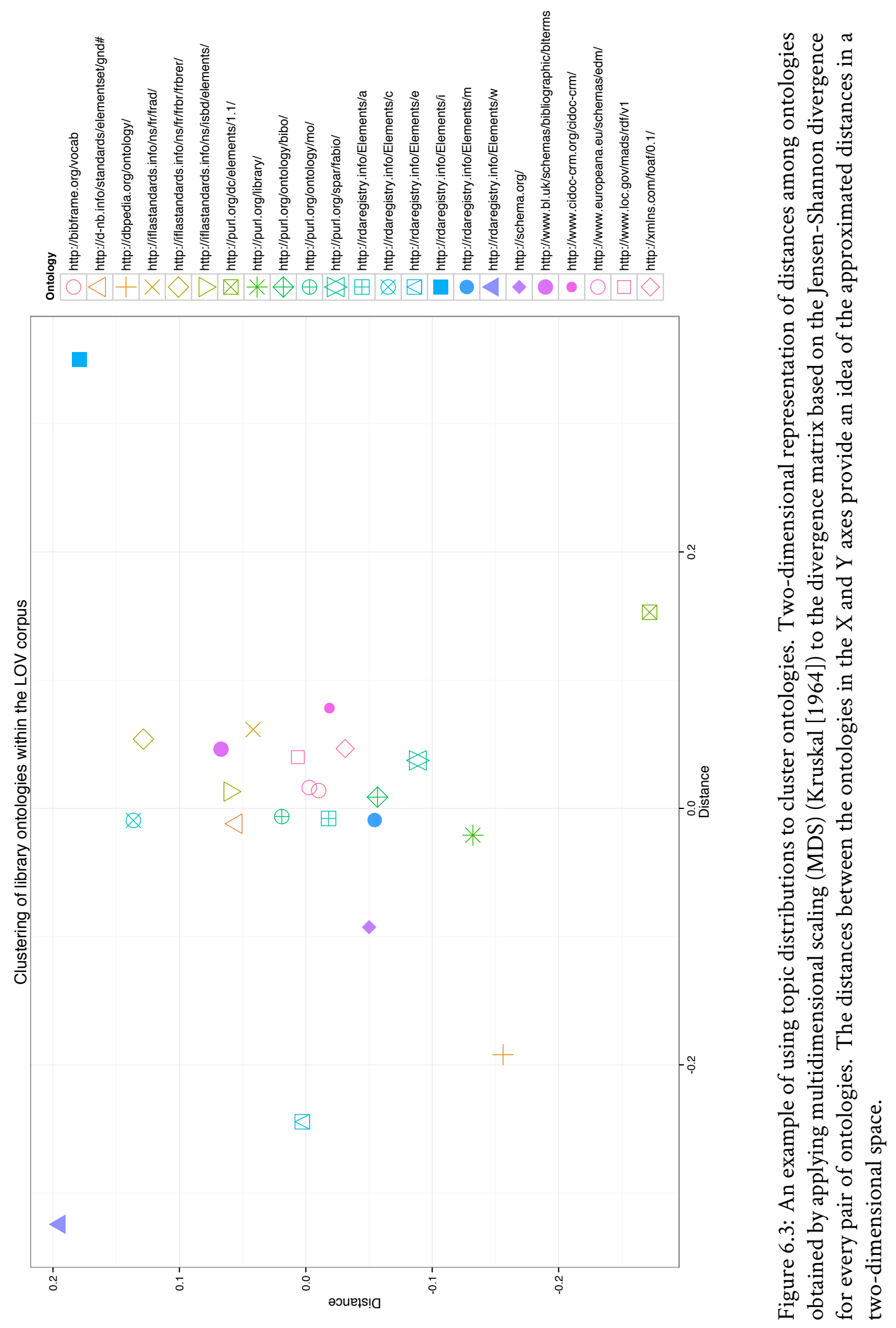


Using the divergence matrix, we show a graphical example of clustering library ontologies. In particular, we applied multidimensional scaling (Kruskal [1964]) to the aforementioned divergence matrix. By applying multidimensional scaling with two dimensions, we obtained a graph that groups together in a two-dimensional space those ontologies that are more similar to each other. We present the results in Figure 6.3.

From the graph in Figure 6.3, the first observation is that the majority of ontologies clearly concentrate in a cluster, indicating that our method correctly identifies that they describe an specific domain of interest (i.e., the library domain). Another observation is that the Europeana Data Model ${ }^{12}$ (EDM) and the BIBFRAME vocabulary are highly related $(J S D=0.03)$. In fact, the EDM and BIBFRAME ontologies take a similar approach in terms of the entities they describe (e.g., agents, aggregations of resources, events, etc.), their granularity, and their focus on maximum coverage of bibliographic and cultural entities. It is worth noting that even when they both use different terminologies (e.g., Instance in BIBFRAME and Cultural object in EDM), marimba-topicmodel is able to identify that they are highly related. This indicates that using topic distributions can provide more sophisticated mechanisms than those based on pure lexical similarity.

Finally, we observe that two ontologies are clearly distant from the others, namely the RDA properties for Works and the RDA properties for Items. By analyzing the topic distribution of the former, we observe that more prominent topics contain senses for terms such as video, film, and instrumentalist. These terms are aligned with the purpose of the vocabulary which is to widely cover different types of creative works, as opposed to more general ontologies to describe works such as IFLA FRBR. Regarding the latter, it is a vocabulary for describing bibliographic items or exemplars by providing physical details, which is a level of granularity barely covered by other library ontologies.

\subsection{Evaluation}

The main objective of marimba-topicmodel is the evaluation of the fourth hypothesis of this thesis (H4): probabilistic topic modelling techniques can produce coherent descriptions of ontologies and can perform better than existing methods for ontology search. However, it can be seen that we build on two initial sub-hypotheses, namely:

\footnotetext{
${ }^{12}$ http://www. europeana.eu/schemas/edm/ (Last viewed 26th April 2016)
} 
H4a The annotation of ontology documents with word senses can improve the coherence of the extracted topics (i.e., word-senses annotation step described in Section 6.4.2).

H4b Sense-annotated ontology documents will suffer from data sparsity issues, and, thus, short-text oriented topic models will produce more coherent topics than classical topic models (i.e., the topic modelling step using BTM described in Section 6.4.3).

In order to validate our main hypothesis and the two initial sub-hypotheses, in this section we carry out and discuss the following two experiments:

Experiment 1. Topic coherence evaluation. We carry out an evaluation of our method using a coherence measure to quantify the coherence of the extracted topics. First, we compare the topic coherence of $i$ ) LDA with non-annotated ontology documents and $i$ i) LDA with sense-annotated ontology documents to test our first sub-hypothesis H4a. Moreover, we compare the aforementioned LDA variants with our proposed topic model, the state-of-the-art topic model for short-text BTM using sense-annotated ontology documents, to test our second sub-hypothesis $\mathrm{H} 4 \mathrm{~b}$.

Experiment 2. Task-based evaluation. We report on a task-based experiment using our method to train a BTM topic model with sense-annotated ontology documents, and a baseline method using tf-idf, which is a recurring technique used by ontology search repositories such as LOV or Falcons. The performance of the methods is tested through an ontology clustering task with a humanannotated gold-standard corpus extracted from the LOV repository and the LODStats dataset. This evaluation tests our fourth hypothesis H4.

\subsubsection{Training corpus}

Although our method can work with any corpus of ontologies, for our experiments we train the topic models with the LOV corpus (Linked Open Vocabularies corpus) ${ }^{13}$, a collection of more than 500 curated and thematicly diverse ontologies. Originally, the corpus contained 511 ontologies, but after applying the lexical extraction step we reduced their number to 504 ontologies, due to parsing issues or inability to extract lexical elements.

\footnotetext{
${ }^{13}$ The complete corpus is available for download at http://lov.okfn.org/lov.n3.gz (Retrieved 12nd February 2016)
} 


\subsubsection{Experiment 1. Topic coherence evaluation}

In order to evaluate the quality of the generated topics, we apply the topic coherence measure proposed by Mimno et al. [2011] for topic quality evaluation. Mimno et al. [2011] empirically showed that this measure is highly correlated with human-judgements and has since then become a standard quality measure for topic models. The underlying idea of this measure is that the semantic coherence achieved by a topic model is related to the co-ocurrence patterns of words describing the topics within the documents of the corpus.

\section{Experimental setting}

In this experiment, we use three different configurations:

- LDA: An LDA implementation trained with non-annotated ontology documents, which means skipping the word-senses annotation step.

- LDA $A_{S}$ : The same LDA implementation ${ }^{14}$ trained with sense-annotated ontology documents.

- marimba: Our contribution, an implementation of the Biterm topic model trained with sense-annotated ontology ontology documents, which corresponds to our proposed marimba-topicmodel method.

The three topic models are configured with standard values ${ }^{15}$ for hyperparameters $\alpha$ and $\beta:(\alpha=(50 / k)+1, \beta=0.1+1)$ for $L D A$, and $L D A_{S}$; and $(\alpha=$ $(50 / k), \beta=0.01)$ for marimba-topicmodel. The results presented in this experiment are the average of ten runs with each method. Finally, we run the experiments with different values for $K$ in order to analyze the impact of the number of topics. It is worth noting that we are not interesting in finding the best configuration for the training corpus, but instead in comparing our method and the proposed baselines under different number of topics.

\section{Topic coherence measure}

In this section, we define the topic coherence measure proposed by Mimno et al. [2011]. Given the document frequency of word $w, f(w)$ (i.e., the number of documents with

\footnotetext{
${ }^{14}$ For $L D A$ and $L D A_{S}$ we use the implementation provided by the Apache Spark framework in its version 1.5.0.

${ }^{15}$ For LDA we use the hyperparameter values recommended by the Apache Spark framework and for BTM the values used in Cheng et al. [2014]
} 
at least one occurence of word $w$ ) and the co-document frequency of words $w$ and $w^{\prime}$ (i.e., the number of documents containing one or more occurrences of $w$ and at least one occurrence of $\left.w^{\prime}\right)$. The topic coherence measure is defined as follows:

$$
c\left(t ; W^{(t)}\right)=\sum_{m=2}^{M} \sum_{l=1}^{m-1} \log \frac{f\left(w_{m}^{(t)}, w_{l}^{(t)}\right)+1}{f\left(w_{l}^{(t)}\right)}
$$

where $W^{(t)}=\left(w_{1}^{(t)}, \ldots, w_{M}^{(t)}\right)$ is a list of the M most probable words in topic t. Please note that a count of 1 is included to avoid taking the logarithm of zero.

Further, given the topic coherence for each topic $t \in K$, we can calculate the average topic coherence for each topic model as follows

$$
C(T ; K)=\frac{1}{K} \sum_{k=1}^{K} c\left(t_{k} ; W^{\left(t_{k}\right)}\right)
$$

where $\mathrm{T}$ is the topic model being evaluated and $\mathrm{K}$ the number of topics.

\section{Results}

In our evaluation, we calculate the average topic coherence for values of $K$ from 10 to 60 and of $M$ from 5 to 20. The results, presented in Figure 6.4, show that marimbatopicmodel consistently outperforms $L D A$ and $L D A_{S}$ for every $K$ and length of the top $\mathrm{M}$ words of the topic and the improvement is statistically significant $(P-$ value $<0.001)$. We discuss these results in Section 6.6.

\subsubsection{Experiment 2. Task-based evaluation}

In this section, we present the results of a task-based evaluation. In particular we would like to measure the precision of our topic-based ontology similarity method by using it to cluster thematicly related ontologies. The underlying idea is that if our topic model produces good quality results, the distribution of topics for each ontology can be used to automatically group and discriminate among topically related ontologies. In this experiment, we compare our method marimba-topicmodel with a baseline method based on tf-idf with different similarity and distance measures.

\section{Gold standards: LOVTAGS and LODTHEMES datasets}

To be able to perform this type of evaluation, we need a dataset that has been annotated with thematic information for each ontology. In this thesis, we propose and create two 

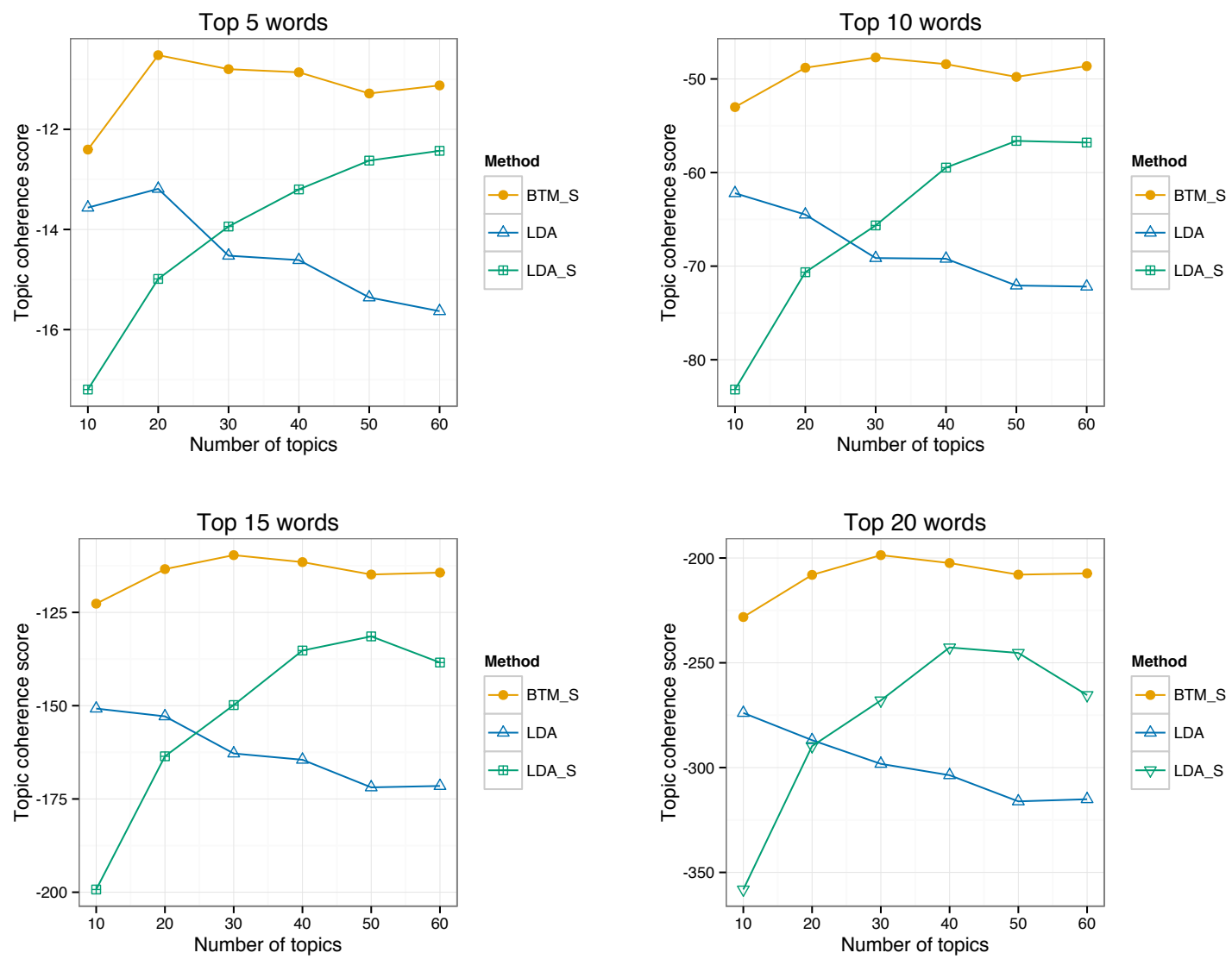

Figure 6.4: Coherence scores $\mathrm{C}(\mathrm{T} ; \mathrm{K})$ results for $L D A, L D A_{S}$ and marimbatopicmodel with $K$ from 10 to 60 . A larger value for the coherence score indicate more coherent topics. The average of ten runs with each method is shown.

gold-standard datasets: LOVTAGS and LODTHEMES. We have extracted these two gold-standard datasets from human annotations of two different datasets, the LOV corpus, and the LODstats ${ }^{16}$ dataset respectively. We introduce the gold-standards below:

LOVTAGS is a corpus containing tags for a subset of the LOV corpus. The tags and their corresponding ontologies have been extracted from the metadata dump available in the LOV website. ${ }^{17}$ It is worth noting that the tags for each ontology have been assigned by the human editors and curators maintaining the LOV repository. The

\footnotetext{
${ }^{16}$ The LODstats dataset is part of an initiative for collecting statistics about LOD datasets, the data is available at http: //stats. lod2. eu/ (Retrieved 12nd February 2016)

${ }^{17}$ http://lov.okfn.org/lov.n3.gz (Retrieved 12nd February 2016)
} 
LOVTAGS gold-standard has the following characteristics:

- We have manually selected those tags that indicate a thematic category and excluded other tags such as W3C Rec for W3C Recommendations, or Metadata for general metadata ontologies. Specifically, the LOVTAGS dataset contains the following 27 tags with the number of member ontologies between brackets: Academy (9), Travel (4), eBusiness (6), Government (10), Society (18), Support (18), Industry (10), Biology (11), Events (12), General and Upper (15), Image (3), Music (6), Press (6), Contracts (6), Catalogs (30), SPAR (12), SSDesk (5), Geography (23), Food (6), Quality (23), Multimedia (12), Health (3), People (20), Environment (9), Methods (36), Security (7), and Time (14).

- We have selected only the ontologies that belong to exactly one tag with the goal of having more clearly delimited thematic clusters. This decision was motivated by the need of having clearly defined clusters of ontologies during the experiments.

LODTHEMES is a smaller dataset containing thematic tags for a subset of the ontologies within the LOV corpus. The main idea for the creation of the LODTHEMES goldstandard was to use the human annotations about themes done by the maintainers of the LOD Cloud diagram. ${ }^{18}$ We have created the dataset by automatically processing the LODstats dataset and the LOV corpus in the following way:

1. For each dataset described in the LODstats dataset, we extracted the annotations about: $i$ ) the ontologies used in each data set of the LOD cloud; and ii) the thematic category. In this way, we associated each ontology with one or more thematic category. As with the LODTAGS dataset, we are interested only in those ontologies belonging to exclusively one category.

2. For each ontology extracted in the previous step, we selected those that were available in the LOV corpus.

3. The resulting LOVTHEMES gold-standard dataset contains the following five themes with the number of member ontologies in brackets: Life-sciences ${ }^{19}(21)$,

\footnotetext{
${ }^{18}$ See online at http://lod-cloud. net (Last viewed 26th April 2016)

${ }^{19}$ http://lod-cloud.net/themes/lifesciences (Last viewed 26th April 2016)
} 
Government $^{20}$ (52), Media $^{21}$ (9), Geographic ${ }^{22}$ (31), Publication ${ }^{23}$ (53).

In summary, the LODTAGS dataset contains 27 thematic clusters and the LODTHEMES dataset contains 5 thematic clusters. In the following section, we describe the measures to be used in our experiment to evaluate the quality of our method for the task of ontology clustering using these two gold-standards.

\section{Measures}

In order to measure the precision of the marimba-topicmodel method in the task of ontology clustering, we propose a measure called $\mathrm{H}$ score, based on the work of Bordino et al. [2010], which quantifies the ability of a method to cluster together similar ontologies. The measure is based on the notion that: $i$ ) ontologies belonging to the same cluster should show a high degree of similarity; and ii) ontologies belonging to different clusters should show a lower degree of similarity. We formalize this notion below by defining the $\mathrm{H}$ score.

Let $G=\left\{G_{1}, \ldots, G_{n}\right\}$ be the set of $n$ clusters defined in a gold-standard dataset, and dis a similarity measure. Given a cluster $G_{n} \in G$, the intra-cluster distance measures the similarity between ontologies within a given cluster and is defined as:

$$
\operatorname{IntraDis}\left(G_{n}\right)=\sum_{\substack{d_{i}, d_{j} \in G_{n} \\ i \neq j}} \frac{2 \operatorname{dis}\left(d_{i}, d_{j}\right)}{\left|G_{n}\right|\left|G_{n}-1\right|}
$$

Applying this measure to every cluster we can evaluate the overall quality of a method:

$$
\operatorname{IntraDis}(G)=\frac{1}{N} \sum_{n=1}^{N} \operatorname{IntraDis}\left(G_{n}\right)
$$

On the other hand, given two clusters $G_{n}, G_{n^{\prime}} \in G$ where $n \neq n^{\prime}$, we can measure the inter-cluster distance as:

$$
\operatorname{InterDis}\left(G_{n}, G_{n^{\prime}}\right)=\sum_{d_{i} \in G_{n}} \sum_{d_{j} \in G_{n^{\prime}}} \frac{\operatorname{dis}\left(d_{i}, d_{j}\right)}{\left|G_{n}\right|\left|G_{n^{\prime}}\right|}
$$

\footnotetext{
${ }^{20}$ http://lod-cloud.net/themes/government (Last viewed 26th April 2016)

${ }^{21}$ http://lod-cloud.net/themes/media (Last viewed 26th April 2016)

${ }^{22}$ http://lod-cloud.net/themes/geographic (Last viewed 26th April 2016)

${ }^{23}$ http://lod-cloud.net/themes/publications (Last viewed 26th April 2016)
} 
In order to evaluate the clustering method with respect to the set of clusters $G$, we define $\operatorname{InterDis}(G)$ as:

$$
\operatorname{InterDis}(G)=\frac{1}{N(N-1)} \sum_{\substack{G_{n}, G_{n^{\prime}} \in G \\ n \neq n^{\prime}}} \operatorname{InterDis}\left(G_{n}, G_{n^{\prime}}\right)
$$

Based on the notion that a effective clustering method will show a smaller value of $\operatorname{IntraDis}(G)$ value with respect to the value $\operatorname{InterDis}(G)$, we define the $H(G)$ score as:

$$
H(G)=\frac{\operatorname{IntraDis}(G)}{\operatorname{InterDis}(G)}
$$

Regarding the similarity measure $d i s$, in this evaluation we experiment with four different measures: $i$ ) the $J S D$ measure defined in Equation 6.2 ; ii) the Cosine distance cos; $i i i)$ the Jensen-Shannon metric JSM; and iv) the Euclidean distance euc. To defined $\cos$ and euc, let $\boldsymbol{x}$ and $\boldsymbol{y} \in \mathbb{R}^{n}$ be two vectors, $\cos (\boldsymbol{x}, \boldsymbol{y})$ and $e u c(\boldsymbol{x}, \boldsymbol{y})$ are defined as follows:

$$
\begin{aligned}
& \cos (\boldsymbol{x}, \boldsymbol{y})=1-\frac{\boldsymbol{x} \cdot \boldsymbol{y}}{\|\boldsymbol{x}\|\|\boldsymbol{y}\|} \\
& \operatorname{euc}(\boldsymbol{x}, \boldsymbol{y})=\sqrt{\sum_{i=1}^{n}\left(x_{i}-y_{i}\right)^{2}}
\end{aligned}
$$

\section{Experimental setting}

In this task-based experiment, we compare the following methods:

- tf-idf: This baseline method generates tf-idf vectors for each sense-annotated ontology document. Specifically, these vectors are generated by using the nonannotated corpus $D$ obtained by applying the ontology document extraction step described in Section 6.3.1.

- marimba: An implementation of the Biterm topic model trained with senseannotated ontology ontology documents, which corresponds to our proposed marimba-topicmodel method.

For each method we calculate the $H$ score using distance measures and distance metrics. Regarding the distance measures, we compare the two methods in the following way: 
- tf-idf-cosine: The tf-idf method using the cosine distance defined in Equation 6.18.

- marimba-cosine: The marimba-topicmodel method using the cosine distance defined in Equation 6.18.

- marimba-jsd: The marimba-topicmodel method using the topic-based ontology similarity measure defined in Equation 6.2.

Regarding the distance metrics, we compare the two methods in the following way:

- tf-idf-euclidean: The tf-idf method using the Euclidean distance defined in Equation 6.19.

- marimba-jsm: The marimba-topicmodel method using the Jensen-Shannon metric defined in Equation 6.4.

The marimba topic model is trained with the following hyperparameters values: $(\alpha=(50 / k), \beta=0.01)$ and $K=30$.

\section{Results}

The results of the experiment, presented in Table 6.2, show that our method, marimbatopicmodel, achieves consistently better $H(G)$ score using similarity measures for the LOVTAGS and LODTHEMES gold-standard datasets, and the best score is achieved using the JSD topic-based ontology similarity (Eq. 6.2). Furthermore, as shown in Table 6.3, the marimba-topicmodel method achieves better $H(G)$ score using distance metrics for the LOVTAGS and LODTHEMES gold-standard datasets. Specifically, the best score is achieved using the JSM metric (Eq. 6.4). We discuss these results in Section 6.6.

\subsection{Discussion}

As an overall observation, we note that the evaluation results are in line with our initial hypotheses, namely: $i$ ) probabilistic topic modelling can be effectively applied to represent ontologies as a mixture of latent features, $i i$ ) applying word-sense disambiguation to annotate ontology contexts reduces lexical ambiguity and increases the quality of the topics, even $L D A_{S}$ achieves better topic coherence than traditional $L D A$ (H4a), and iii) our marimba-topicmodel method using BTM mitigates the 
Table 6.2: Results of the ontology clustering experiment for our task-based evaluation using distance measures. The value of the $\mathrm{H}$ score $\mathrm{H}(\mathrm{G})$ for each method and goldstandard dataset are shown. Lower values of the $\mathrm{H}$ score indicate that the method is more precise. For marimba-topicmodel the average of ten runs is shown, with the standard deviation in brackets.

\begin{tabular}{lll}
\hline Method & LOVTAGS $(\mathrm{H}(\mathrm{G}))$ & LODTHEMES $(\mathrm{H}(\mathrm{G}))$ \\
\hline tf-idf-cosine & 0.924 & 0.842 \\
\hline marimba-cosine & $0.861(\mathrm{sd}=0.009)$ & $0.722(\mathrm{sd}=0.034)$ \\
\hline marimba-jsd & $0.859(\mathrm{sd}=0.009)$ & $0.683(\mathrm{sd}=0.11)$ \\
\hline
\end{tabular}

Table 6.3: Results of the ontology clustering experiment for our task-based evaluation using distance metrics. The value of the $\mathrm{H}$ score $\mathrm{H}(\mathrm{G})$ for each method and goldstandard dataset are shown. Lower values of the $\mathrm{H}$ score indicate that the method is more precise. For marimba-topicmodel the average of ten runs is shown, with the standard deviation in brackets.

\begin{tabular}{lcc}
\hline Method & LOVTAGS $(\mathrm{H}(\mathrm{G}))$ & LODTHEMES $(\mathrm{H}(\mathrm{G}))$ \\
\hline tf-idf-euclidean & 0.808 & 0.998 \\
\hline marimba-jsm & $\mathbf{0 . 7 2 3}(\mathrm{sd}=0.008)$ & $\mathbf{0 . 7 4 1}(\mathrm{sd}=0.037)$ \\
\hline
\end{tabular}

data sparsity issues with sense-annotated ontology documents, achieving the best results for every experiment (H4b).

\subsubsection{Topic coherence}

Regarding topic coherence, the results highlight that marimba-topicmodel shows not only the best scores for every combination, but also that is the most stable. This stability lies in the fact that it models the generation of biterms within the whole corpus as opposed to LDA that models the document generation process. Although the results are very positive, additional evaluation should be performed eventually involving human annotators or applying other coherence metrics, extending for example those proposed by Lau et al. [2014].

Another aspect to highlight from the results is that $L D A$ is outperformed by $L D A_{S}$ starting from $K=20$ which seems to indicate that annotating with senses can help to reduce data sparsity issues; although this would need to be verified with 
additional evaluations focusing on measuring this question.

\subsubsection{Task-based evaluation: LOVTAGS and LODTHEMES}

Regarding the task-based evaluation, the results show that marimba-topicmodel consistently achieves the best average ratio $H(G)$ among the evaluated methods for both similarity measures and distance metrics. Additionally, to identify potential weaknesses of our approach, we perform a qualitative evaluation. In particular, we analyze the Intra-cluster distance for each cluster.

In terms of a more qualitative evaluation, we analyzed the Intra-cluster distance for each cluster in the LOVTAGS gold standard. On the one hand, we observe that clusters such as e-business, multimedia, or health achieve very good intra-cluster scores (e.g., in the order of 0.01 in the case of e-business and health).

On the other hand, the tag geography achieves poor scores that are close to 1. By analyzing the data, we find that there are a number of ontologies with a very low score that could not be correctly modeled by marimba-topicmodel due to the fact that they contain textual descriptions in languages other than English (e.g., Norwegian, French, or Spanish). This points to the problem of multilingualism, a limitation of this work and a future line of research. The WSD method that we use can annotate senses in different languages, which make this a natural step. Apart from these ontologies, we find that thematicly related geography vocabularies are close to each other and, in fact, marimba-topicmodel can separate in different clusters ontologies that describe administrative units to those that describe geo-spatial features. To illustrate this, we applied the same technique which we showed in Section 6.4.4 (i.e., multidimensional scaling) to the set of ontologies tagged as Geography in the LOV corpus. We show the result of applying this technique in Figure 6.5. In the figure, those ontologies that contain descriptions that are not in English, are the ones that are less closer to the rest of the ontologies. In particular, the Geo-deling ${ }^{24}$ ontology, on the right side of the figure, is described in Norwegian, and the Geodan ontology, on the top center side of the figure, is a localized ontology from the Netherlands. Finally, it is worth noting that other techniques that rely on the bag of words assumption (e.g., tf-idf) will suffer from the same issues related to multilingualism, and our WSD-based solution is better prepared to deal with these issues by disambiguating to senses that are shared accross languages.

\footnotetext{
${ }^{24}$ http: //vocab. lenka.no/geo-deling (Last viewed 24th May 2016)
} 


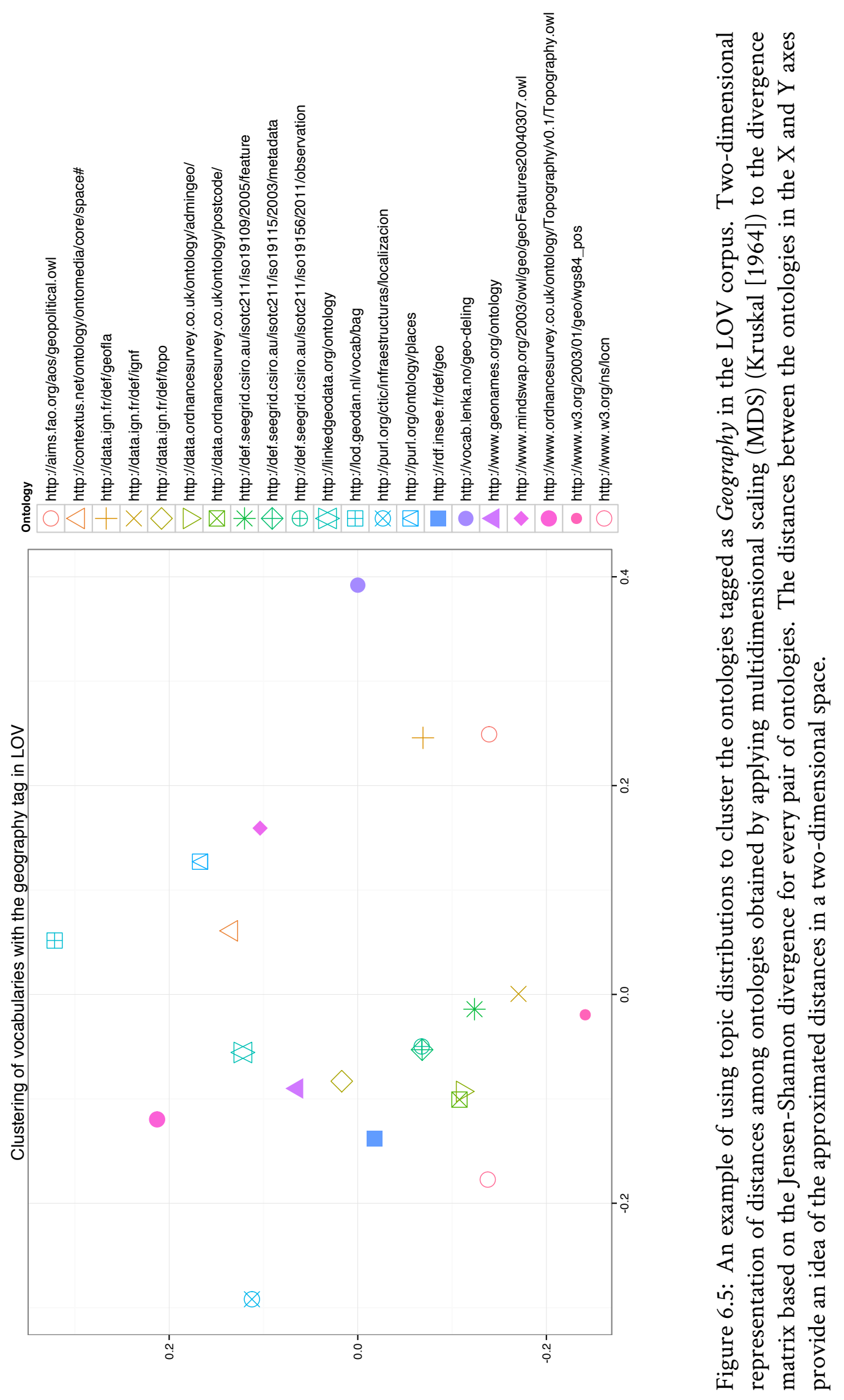




\subsection{Summary}

We have explored the task of modelling topics within ontologies. We have proposed a novel method that extracts the lexical elements of the ontologies, annotates them with external senses, connected to the LOD cloud, and we have used these annotated documents to train a probabilistic topic model. Moreover, we have evaluated three topic models, traditional $L D A, L D A_{S}$ and marimba-topicmodel, in order to investigate the suitability of our method for topic modelling of ontologies. Our findings reveal that marimba-topicmodel consistently outperforms the other approaches, and produces coherent topics that can be applied to tasks such as ontology clustering or enhancing ontology search. This evaluation validates our fourth hypothesis H4. Accompanying the method, we release two evaluation gold-standards, LOVTAGS and LODTHEMES, as well as the implementation of the measures and metrics used, which can be used to evaluate further approaches. 


\section{Generation and publication: datos.bne.es}

Motivated by the growing interest in LOD and semantic technologies, in 2011 the National Library of Spain (BNE) and the Ontology Engineering Group from "Universidad Politécnica de Madrid” started the "datos.bne.es" with the goal of transforming the authority and bibliographic catalogues into RDF following linked data best practices. The technical contributions presented in this thesis have been extensively applied in the context of this project. Moreover, the methodological guidelines presented in this chapter have been widely applied in other domains such as the linguistic, meteorological (Atemezing et al. [2013]) and building construction (Radulovic et al. [2015]) domains. In particular, the methodological guidelines presented in this chapter have been published in Vila-Suero and Gómez-Pérez [2013], Vila-Suero et al. [2013], VilaSuero et al. [2014a], and Gracia et al. [2014b].

In this chapter, we describe and discuss the application of the methods, models and constructs proposed in this thesis within the datos.bne.es project. First, we provide an overview of the project and its main phases (Section 7.1). Second, we introduce the methodology applied to generate, publish and consume the linked dataset of the datos.bne.es project (Section 7.2). Third, from Section 7.3 to Section 7.7 we describe the main activities of the methodology and their application to the datos.bne.es project. Finally, in Section 7.8, we give an overview of the main design and technical features of the datos.bne.es service, which exploits the datos.bne.es dataset to provide the enduser and machine-oriented data service at http://datos.bne.es. 


\subsection{Overview of the datos.bne.es project}

Started in 2011, the datos.bne.es project has been developed in the following phases:

Cervantes dataset (2011) The initial phase of the project consisted in the transformation of a subset of the catalogue into RDF, modelled with the ontologies developed by the IFLA. During this phase, the team explored different techniques for mapping and extracting entities and relationships out of records in the MARC 21 standard. The data sources transformed during this phase included the works authored by "Miguel de Cervantes Saavedra", the publications related to these works, the "authorities" (persons, organizations and subjects) related to these publications, and finally, the works related to these authorities. The data sources comprised 8,552 records in the MARC 21 bibliographic format and 41,972 record in the MARC 21 authority format. The RDF dataset was modelled using several classes and properties from the IFLA FRBR, FRAD, and ISBD ontologies. The RDF dataset produced during this phase was not published on the Web. During this initial phase, the need for the active participation of library experts during the mapping process was identified, which led to the design and development of the constructs, models, and methods presented in this thesis. The results of this phase are described in Vila Suero and Escolano Rodríguez [2011].

datos.bne.es initial dataset (2012-2013) The second phase of the project consisted in the generation and publication of a significant part of the catalogue following the linked data principles. The results of this phase are described in Vila-Suero et al. [2013]. The main result of this phase was the release of a large and highly interlinked RDF dataset under the public domain Creative Commons $\mathrm{CCO}^{1}$ license. The data sources transformed consisted in 2,390,103 bibliographic records and $2,028,532$ records authority records. During this phase a first prototype of the marimba-framework was designed and developed. This first prototype allowed domain experts to actively participate in the mapping and ontology development process described in Chapter 4. The ontology used for transforming the data sources into RDF was the FRBR-based ontology described in Chapter 5. Regarding the publication on the Web, the dataset was made available in two different ways: $i$ ) through a public SPARQL endpoint ${ }^{2}$, and $i$ ) through a stan-

\footnotetext{
${ }^{1}$ https: //creativecommons.org/about/cc0/ (Last viewed 3rd May 2016)

${ }^{2}$ http://datos.bne.es/sparql
} 
dard linked data front-end, Pubby ${ }^{3}$, which provided access to the data in different formats using content-negotiation. Regarding the links to external datasets, the RDF dataset produced during this phase included 587,521 owl : sameAs links to the following datasets: $\mathrm{VIAF}^{4}, \mathrm{GND}^{5}, \mathrm{DBpedia}, \mathrm{Libris}^{6}$, and SUDOC ${ }^{7}$. At this stage the marimba-tool prototype did not make use of the marimba-rml and marimba-sql languages for mapping and transforming the data sources into $\mathrm{RDF}$. Instead, the prototype relied on a programmatic transformation using an early version of the mapping templates described in Chapter 4. Finally, during this phase the methodological process for transforming library catalogues into RDF was defined and described in Vila-Suero and Gómez-Pérez [2013].

datos.bne.es 2.0 (2014-2015) The current phase of the project, started in 2014, corresponds to the dataset and the service that is described in this chapter and will be used for the user-centered evaluation presented in Chapter 8. The main characteristics of this phase are summarized as follows:

- The National Library catalogue was transformed and interlinked covering 4,784,303 bibliographic records and 3,083,671 authority records to generate 143,153,218 unique RDF triples. Moreover, the number of owl:sameAs links to external datasets was increased up to 1,395,108 links. Additionally, 108,834 links to digitalized materials were added.

- The data was modelled using the BNE ontology described in Chapter 5. The BNE ontology was published following the linked data principles. ${ }^{8}$

- An end-user online service was developed to give access to the vast amounts of interconnected entities. This user interface leverages the RDF data and the underlying BNE ontology to index, present, and arrange information. As of July 2016 more the service has received more than 1 million visitors with an average of 45,000 visitors per month.

\footnotetext{
${ }^{3}$ The front-end used by projects like DBpedia

${ }^{4}$ The Virtual International Authority File

${ }^{5}$ The Authority file of the German National Library

${ }^{6}$ The authority and bibliographic catalogues of the National Library of Sweden

${ }^{7}$ The French Union Catalogue for Public Universities

${ }^{8} \mathrm{http}: / /$ datos.bne.es/def/
} 


\subsection{Methodology}

In this section, we provide an introduction to the methodology followed for the generation and publication of the datos.bne.es dataset. Over the last years, several methodological guidelines for generating and publishing linked data have been proposed (Heath and Bizer [2011b], Villazón-Terrazas et al. [2011], Hyland et al. [2014]). These methodological guidelines provide a principled way for generating, publishing and eventually consuming linked data through a series of activities whose objective is to produce high quality linked data. However, existing guidelines present two major limitations with respect to their application in the library domain. First, as we argued in VillazónTerrazas et al. [2012], a general application of existing guidelines to every domain is not possible, due to the intrinsic characteristics of every use case and domain of knowledge. Second, as we argued in Vila-Suero et al. [2014a], existing guidelines do not sufficiently address the multilingual aspects of the generation, publication, and exploitation of linked data. Therefore, in this thesis we present an extension of existing guidelines to: $i$ ) adapt them to the library domain, and $i i$ ) include methodological guidelines that account for multilingual features of linked data. The methodology proposed in this thesis is composed of seven activities:

1. Specification: The goal of this activity is to analyze and specify the data sources to be transformed into RDF and the identifiers of the RDF resources to be produced during the RDF generation activity. This activity comprises two tasks: $i$ ) identification and analysis of data sources, and ii) URI and IRI design. This activity is described in Section 7.3.

2. Data curation: The goal of this activity is to analyze, identify and potentially fix existing issues in the data sources. This activity is divided into two tasks: $i$ ) identifying issues, and $i i$ ) reporting and fixing. This activity is described in Section 7.6.

3. Modelling: The goal of this activity is to design and develop the ontology to be used for modelling the data sources to be transformed into RDF. This activity comprises the ontology development methodology that has been already presented in Chapter 5.

4. RDF generation: The goal of this activity is to transform the data sources into RDF using the ontology developed during the modelling activity. This activity 
deals with the mapping of the data sources using the ontology developed during the modelling activity. This activity is described in Section 7.4.

5. Data linking and enrichment: The goal of this activity is to identify and establish links to external linked datasets. This activity comprises three tasks: $i$ ) identification and selection of target datasets, $i i$ ) link discovery, and iii) link validation. This activity is described in Section 7.5.

6. Publication: The goal of this activity is to make the dataset available on the Web following the linked data principles and best practices. This activity comprises two tasks: $i)$ data publication, and ii) dataset metadata publication. This activity is described in Section 7.7.

7. Exploitation: The goal of this activity is to develop applications that consume the published linked data to provide services for end-users and/or third-party applications. This activity is described in Section 7.8.

\subsection{Specification}

The goal of this activity is to analyze and describe the data sources and the identifiers of the RDF resources to be produced during the $R D F$ generation activity. This activity is composed of two tasks (Identification and analysis of data sources and IRI design) that we describe below.

\subsubsection{Identification and analysis of data sources}

The goal of this task is to identify and describe the data sources to be transformed into RDF. Specifically, at least the following features of the data sources should be documented: $i$ ) data formats, $i$ ) access mechanisms, and $i i i)$ main concepts described in the data sources. In Table 7.1, we provide an overview of the features of the data sources for the datos.bne.es 2.0 dataset.

\subsubsection{IRI and URI design}

The goal of this task is to design the structure of the URIs and IRIs that will be used to uniquely identify the resources to be generated. When designing URIs, it is advisable to follow well-established guidelines, such as those presented in the articles "Cool 
Table 7.1: Description of the data sources for datos.bne.es 2.0 dataset.

\begin{tabular}{|l|l|}
\hline Data formats & MARC 21 bibliographic and authority formats \\
\hline Access mechanisms & Files in the ISO2709 encoding \\
\hline \multirow{3}{*}{ Main concepts } & $\begin{array}{l}\text { Persons, Organizations, Conferences, Subjects, } \\
\text { Works, Translations of Works, Maps, Manuscripts, Books, } \\
\text { Software, Musical scores, Sound and Audiovisual recordings }\end{array}$ \\
\hline
\end{tabular}

URIs don't change" and "Cool URIs for the Semantic Web"10, the guidelines "Designing URI sets for the UK public sector" ${ }^{11}$, the study on Persistent URIs by the ISA (Interoperability Solutions for European Public Administrations) programme ${ }^{12}$, or the Linked Data patterns online book. ${ }^{13}$ From these guidelines, we have extracted five features that should be taken into account when designing IRIs for the library domain: $i$ ) namespace, $i$ ) type of identifiers, $i i i)$ URI/IRI strategy, iv) content-negotiation, and $v$ ) readability. We describe each of these features and the available options below. Namespace. The first feature to be defined when designing the IRIs for publishing linked data is the namespace under which the created resources will be available. The main recommendation is that the namespace should be owned and maintained by the organization publishing the data. In the case of the datos.bne.es project, the namespace corresponds to the subdomain "datos" (data in Spanish) under the main domain of the BNE (i.e., bne.es).

Type of identifiers. The main recommendation is to separate the identifiers at least into two types: $i$ ) ontology identifiers, which are used for uniquely identifying the classes and properties of the ontology to be developed in the Modelling activity, and ii) instance identifiers, which are used to identify the RDF resources to be generated as instances of the ontology in the RDF generation. In the case of the datos.bne.es project, we separated the ontology and instance identifiers in the following way. For the ontology identifiers, we followed the recommendation made by the ISA guidelines and used the following base URI: http://datos.bne.es/def/. For instance identifiers,

\footnotetext{
${ }^{9}$ https://www.w3.org/Provider/Style/URI (Last viewed 3rd May 2016)

${ }^{10}$ https: //www.w3.org/TR/cooluris/ (Last viewed 3rd May 2016)

${ }^{11}$ https://www.gov.uk/government/publications/designing-uri-sets-for-the-uk-public-sector (Last viewed 3rd May 2016)

12 https://joinup.ec.europa.eu/community/semic/document/ 10-rules-persistent-uris (Last viewed 3rd May 2016)

${ }^{13}$ http://patterns.dataincubator.org/book/ (Last viewed 3rd May 2016)
} 
we followed the pattern used by well-known projects such as DBpedia and used the following base IRI: http: //datos. bne.es/resource/.

URI/IRI strategy. There are two basic strategies of URIs/IRIs. The first strategy is called the hash IRI, in which a URI contains a fragment that is separated from the rest of the URI by a hash character ('\#'). In the case of hash IRIs, every time a client requests the resource the whole file or document containing the resource is retrieved from the server, which makes this approach costly for long files. Therefore, this approach is only suitable for small ontologies and datasets. The second strategy is known as Slash ('/) IRIs, which implies an HTTP 303 redirection to the location of a document that represents the resource and uses content-negotiation. In this case, resources can be accessed individually or in groups, which makes this approach more suitable for large ontologies and datasets. In the datos.bne.es project, the ontology and instance identifiers follow the Slash ('/) IRIs form.

Content-negotiation. Content-negotiation refers to an HTTP mechanism that provides the ability of requesting and serving different formats and versions of the same web resource. In the context of the Semantic Web, this mechanism is highly used for serving different serializations of an RDF resource (e.g., RDF/XML and Turtle) as well as human-readable descriptions (e.g., HTML). The most widely used contentnegotiation mechanism is to use an HTTP 303 redirection indicating the location in the HTTP server of the document describing the requested resource. In the datos.bne.es project, we use the HTTP 303 content-negotiation strategy and provide resource serializations in Turtle, RDF/XML, JSON-LD and HTML.

Readability. Regarding the readability of the identifiers, there are two approaches available. The first approach is known as meaningful or descriptive identifiers, which uses natural language descriptions to identify the resource in the IRI. An example of a descriptive URI is the class Expression ${ }^{14}$ in the FRBR core ontology, which uses the term Expression directly in the IRI. The second approach is known as opaque identifiers, which uses non-human readable codes to identify the resource in the IRI. An example of an opaque URI is the class Expression ${ }^{15}$ in the IFLA FRBR ontology, which uses the alpha-numerical code $\mathrm{C} 1002$ as identifier. The main advantage of the descriptive identifiers approach is that the URI/IRI is human readable and can help to

\footnotetext{
${ }^{14}$ http://vocab.org/frbr/core\#Expression

${ }^{15} \mathrm{http} / / /$ iflastandards.info/ns/fr/frbr/frbrer/C1002
} 
understand the resource being described by the identifier. However, as discussed in "Cool URIs don't change" and Vila-Suero et al. [2014a], identifiers should not change and descriptive identifiers are less stable and tend to change over time. Moreover, it has been argued (Montiel-Ponsoda et al. [2011]) that "opaque URIs" are more stable and suitable for multilingual scenarios as they can be used to avoid language biases (i.e., choosing one language over others to define the identifier). A notable example of the use of "opaque URIs" are the multilingual ontologies maintained by the IFLA (Montiel-Ponsoda et al. [2011]). In the datos.bne.es project, we used "opaque URIs" for both instance and ontology identifiers, motivated by the multilingual nature of both the data and the BNE ontology.

\subsection{RDF Generation}

The goal of this activity is to map and transform the data sources into RDF using the ontology developed during the modelling activity. This activity is composed of two tasks (Configuration of RDF generation technologies and Mapping sources into RDF) that we describe below.

\subsubsection{Configuration of RDF generation technologies: marimba-tool}

A key decision in the RDF generation activity is the choice and configuration of technologies to be used for mapping and transforming the data sources. In the context of this thesis, in Chapter 4, we have proposed marimba-mapping, that is composed of a schema extraction method to analyze the data sources in MARC 21 and extract the schema in the marimba-datamodel, the marimba-rml mapping language, the marimba-sql query language, and the mapping template generation method. In the context of the datos.bne.es project, we have developed the marimba-tool which implements the constructs, methods and techniques proposed by marimba-mapping. The marimba-tool has been used in production for the datos.bne.es project since 2013 and is licensed to the BNE to support the data transformation for the datos.bne.es service. The marimba-tool is entirely developed in Java $8^{16}$ and, as shown in Figure 7.1, is organized into six modules, which we describe below.

\footnotetext{
${ }^{16}$ https://docs.oracle.com/javase/8/ (Last viewed 04-05-2016)
} 


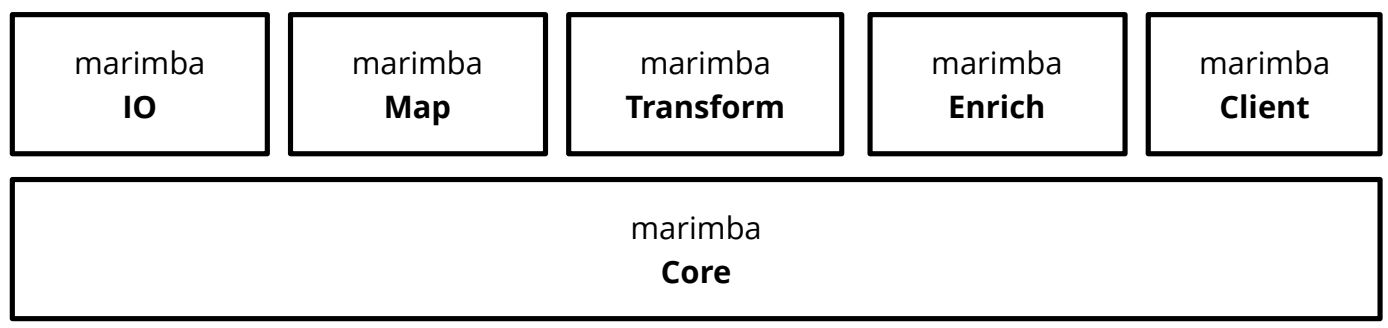

Figure 7.1: marimba-tool modules.

\section{Marimba Core}

This module defines the core architecture and data processing model of marimba-tool. The module provides the interfaces and the core API for the other marimba modules and can be used by third-party applications as an independent library. In particular, the module defines the following components:

1. Model. This component defines the data model of the objects to be processed within the marimba-framework. Specifically, this data model corresponds to the marimba-datamodel based on the nested relational model described in Chapter 4.

2. Parse. This component defines the interface of a generic parser of a data source into objects modelled using the marimba-datamodel. For example, the marimbatool provides an implementation of a parser for MARC 21 files, which parses files containing MARC 21 records and produces a list of objects in the marimbadatamodel. In order to add new formats such as JSON file, a user of the Marimba Core library would only need to implement the generic parser interface to read JSON objects and produce a list of objects in the marimba-datamodel. This feature allows for a flexible integration of new data formats.

3. Processor. This component defines the interface of a generic data processor that receives a list of objects in the marimba-datamodel and produces a list of objects of any type. For example, the Marimba Transform module implements this interface with a processor that receives a list of objects in the marimbadatamodel and produces a list of RDF statements.

4. Persistence. This component defines the interface of a generic data persistor. A data persistor is responsible for storing the output of a data processor. A key design feature of the marimba data processing model is to enable in-memory and 
paralell data processing. Therefore, a data persistor collects objects in-memory and store them in disk periodically. An example of a data persistor, is the $R D F$ persistor implemented by the Marimba Transform module that collects lists of RDF statements and stores them periodically in an RDF repository. ${ }^{17}$

5. Metaprocessor. This component defines the data processing flow of marimba. The metaprocessor is configured with three objects: a parser, a processor and a persistor. Using these three objects the metaprocessor manages the data transformation and storage by parsing, processing and storing the results. The metaprocessor can be configured with a "bucket size", which indicates the size of the list of objects to be processed in parallel, and a "storage ratio", which indicates the size of the list of objects to be collected in-memory before storing them.

\section{Marimba IO}

This module provides methods for importing and exporting data in different formats. The current version of the tool provides methods for $i$ ) importing files in RDF/XML, JSON-LD and Turtle into RDF repositories, and $i$ ) exporting data from RDF repositories into RDF/XML, JSON-LD and Turtle.

\section{Marimba Map}

This module is in charge of modelling and managing the mapping constructs, methods and techniques of the marimba-framework. In particular, the module defines the following components:

1. Model. This component defines the interfaces and classes to model the marimbarml language presented in Chapter 4.

2. Schema. This component implements the schema extraction method, presented in Chapter 4, which is implemented using a MARC 21 parser to parse the MARC 21 data sources, a processor to extract the schema information from the objects produced by the parser, and an $R D F$ persistor to store the schema in an RDF repository. This component also provides methods for reading and loading an existing schema from an RDF repository.

\footnotetext{
${ }^{17}$ The current implementation of the tool uses Apache Jena TDB as RDF repository (http: //jena. apache.org/documentation/tdb/index. html Last viewed 04-05-2016)
} 
3. Mapping. This component provides methods for reading and writing the mapping rules in two formats: $i$ ) mapping templates, which correspond to the mapping template generation method described in Section 4.6, and ii) marimbarml mapping files following the language syntax described in Section 4.5.

\section{Marimba Transform}

This module implements the generic marimba-rml mapping processor presented in Chapter 4. This module processes the MARC 21 data sources, using the mapping rules defined by the domain experts and the ontology engineers, and produces RDF data. More specifically, this module uses

- a MARC 21 parser to parse the MARC 21 data sources,

- a Mapping Processor which uses the components of the Marimba Map module to transform the objects produced by the parser into RDF statements using the marimba-rml mapping rules,

- an $R D F$ persistor to store the generated RDF statements.

It is worth noting that, in order to transform new types of data such as JSON, a developer would only need to implement the generic parser interface to read JSON objects and produce a list of objects in the marimba-datamodel. These objects can then be processed by the Marimba Transform module. In this way, by handling generic objects in the marimba-datamodel, the processor is completely decoupled from the MARC 21 format.

\section{Marimba Enrich}

This module provides methods for creating links and integrating data from external RDF data sources.

Linking. The current version of the tool provides a method for generating links to VIAF and the library organizations that are included in the VIAF mappings. The VIAF mappings are available for download and include mappings between VIAF and several library organizations world-wide including BNE, BNF, and Library of Congress, among others. The Marimba Enrich module parses the VIAF mappings and extracts the links from the generated RDF resources to VIAF and the other library organizations. We will describe this process in more detail in Section 7.5. 
Enrichment. The tool currently provides a configurable mechanism to enrich the generated RDF resources with external RDF datasets. Specifically, the module can be configured with a path (either in the local file system or a web URL), and the datatype properties to be used for enriching the generated RDF resources. Using this configuration, the module is able to add external properties to the generated RDF resources. For example, for the datos.bne.es, the module is configured to include the properties $\mathrm{dbp}$ : abstract and dbp: thumbnail from the DBpedia data dumps. In this particular case, the module includes these properties to those RDF resources that contain links to DBpedia.

\section{Marimba Client}

This module provides a user-facing interface for excuting the different methods offered by the marimba-tool. This module provides an API for executing commands and can be integrated with different web Services or to provide a graphical user interface for interacting with the marimba-framework. The current version of the tool provides a command-line interface to execute the following commands: $i$ ) extract or update the schema from MARC 21 data sources, $i i$ ) transform MARC 21 data sources into RDF, and iii) import and export RDF data into different formats (using the Marimba IO module).

\subsubsection{Mapping sources into RDF}

This tasks deals with the creation of mapping rules from the MARC 21 data sources into RDF using the ontology developed during the Modelling activity. This taks is performed by the domain experts with the assistance of the ontology engineers. The domain experts can modify the mapping templates to add new mappings from MARC 21 metadata elements to RDF, and execute the marimba-tool using the command provided by the Marimba Client module. Moreover, if new MARC 21 data sources are added or updated, the domain experts can run the command for updating the schema and the updates will be reflected in the mapping templates.

\subsection{Data linking and enrichment}

The goal of this activity is to generate links from the generated RDF dataset to other RDF datasets in order to allow the data consumers to discover additional data from external data sources. Moreover, in this activity, relevant data from other RDF datasets 
can be integrated within the generated RDF resources. In the context of this thesis, we call this task data enrichment. The activity is decomposed into four tasks: $i$ ) identifying target datasets for linking; $i$ ) discovering the outgoing links; iii) validating the outgoing links; and, iv) data enrichment.

\subsubsection{Identifying target datasets for linking}

The goal of this task is to identify datasets of similar topics or general datasets that can provide extra information to the dataset. The datasets can be looked up through data catalogs such as datahub.io or datacatalogs.org.

In the datos.bne.es project, we have focused on linking authority data (Persons, Corporate Bodies, Works, and Expressions). In the library domain, the most complete data source for authority data is the VIAF dataset. Moreover, VIAF makes the "VIAF mappings dataset" available online. This dataset contains links accross several library organizations world-wide.

\subsubsection{Discovering and validating the outgoing links}

The goal of this task is to discover similar entities in the target datasets. There are several tools for discovering links between datasets, such as the SILK framework (Isele et al. [2011]), or Limes (Ngonga Ngomo and Auer [2011]). For a complete survey on data linking methods and tools we point the reader to the survey from Ferrara et al. [2013].

Although these tools can be used to discover links, the validation of potential links poses a challenge for large datasets like the datos.bne.es dataset. Therefore, in the datos.bne.es project, we have initially focused on reusing the VIAF mappings to generate the links to external data sources. Regarding the validation of links, in datos.bne.es we have relied on the quality of the links maintained by VIAF.

For generating the links, the Marimba Enrichment module benefits from the fact that libraries have published their authority files by means of natural keys in order to build the URIs of their RDF resources. Therefore, marimba generates the links by parsing the VIAF mapping file and prepending the namespaces to the different keys found in the file. For instance, we know that GND URIs follow the pattern gnd: \{GNDID $\}$ and that BNE URIs, the pattern bne: \{BNE-ID\}. Using these two URI patterns, we can establish links between datos.bne.es and GND by creating owl:sameAs statements with GND-ID and BNE-ID pairs found in the VIAF links file. 
In this way, the GND-ID 11851993X found in the same VIAF cluster as the BNEID XX1718747 can be used to create the RDF statement about Miguel de Cervantes shown in Listing 7.1.

Listing 7.1: Example of owl: sameAs links for Miguel de Cervantes

Qprefix bne: <http://datos.bne.es/resource/> .

aprefix gnd: <http://d-nb.info/gnd/> .

bne:XX1718747 owl: sameAs gnd:11851993X

\subsubsection{Data enrichment}

The goal of this task is to use existing links to external RDF datasets to integrate relevant data $\mathrm{RDF}$ within the RDF resources generated during the $R D F$ generation activity. The main idea is to include a copy of certain data elements directly in the generated $\mathrm{RDF}$ resources and not relying on external RDF datasets to provide these data. The rationale for this data enrichment approach is two-fold: $i$ ) to provide fast and reliable access to critical data elements, and $i i$ ) to use these data elements for data consumption such as indexing and ranking documents in a search engine.

In the datos.bne.es project, we used the Marimba Enrichment module to integrate two properties from DBpedia data dumps: $d b p$ : abstract and dbp : thumbnail. These two properties are extensively used by the datos.bne.es user interface and search engine that will be presented in Section 7.8.

\subsection{Data curation}

The goal of this activity is to assess and ensure the quality of both the data sources and the generated RDF. Since data quality in the original data sources has a direct impact on the quality of the RDF generated, data curation is a crucial activity in the early stages of the linked data generation process. Therefore, one of the main contributions of our approach is to propose data curation as an activity to be carried out in parallel with the specification, modeling and generation activities.

The task of data source curation is decomposed into three-subtasks: $i$ ) identifying data issues; ii) reporting data issues; and iii) fixing data issues. 


\subsubsection{Identifying data issues}

The RDF generation process, concerning the application of semantically richer models (e.g. FRBR) and the participation of cataloguing experts, brings a good opportunity to assess, report and fix issues in the MARC 21 data sources. During the datos.bne.es project, we have identified the following types of issue:

Coding errors. The most common issue that emerged from the mapping templates was the incorrect use of certain MARC 21 subfield codes. For instance, in the first iteration the classification mapping template showed the combination of subfields $100 \$ a \$ f$ and provided an example (\$a Chicin, Fred, \$f (19542007)). The librarians were able to identify this incorrect use (note that the correct subfield code is $\$ d$ and that $f$ is the starting character of fechas - dates in Spanish). Other examples of problematic issues found were the following: the absence of subfield codes (e.g. 100 \$Astrain, Miguel María), or the absence of subfield delimiters (e.g. 100 \$aMoix, Llàtzer, \$d1955-tLa Costa Brava, \$1 Catalán).

Format errors. This type of issue is related to the format and encoding of MARC 21 records. In this regard, two issues were found: first, the content of certain records was not correctly encoded (e.g. 100 \$1 EspaÛol); and second, the usage of content designators did not comply with the MARC 21 format specification (e.g. a high number of records contained an indicator in the field 001).

Issues derived from the application of FRBR. Perhaps the most interesting type of issues was related to the application of FR models. In this regard, the most relevant issues found were the following:

Non-compliant records according to FRBR. For instance, in the classification mapping for the field 100 , the combination $\$ a \$ 1$ could not be classified into any of the FRBR entities. The mapping revealed that the language subfield (i.e., \$1) was used for including the title information (i.e. \$t) as shown in the following example: \$a Geary, Rick, \$1 A treasure of Victorian murder.

Authority control issues. These issues arose especially during the interrelation step of the mapping process. Specifically, these issues were related to problems concerning the linking of the manifestations to their respective expressions. For instance, several thousands of bibliographic records 
could not be linked to their expression. After evaluation, it was found that there was no authority record created in the authority catalogue for the expression of a work in the original language (e.g., an expression of Don Quijote in Castilian, the original language).

\subsubsection{Reporting and fixing data issues}

In order to report coding errors, format errors, and non-compliant records, the marimba tool automatically generates reports for those content designators that were identified as errors by the librarians in the mapping templates. The report included the list of record identifiers (field 001) classified by the error that was found. In total, the marimba-tool has reported issues on more than two thousand authority records, and more than twenty thousand bibliographic records, during the early stages of the project. The list of record identifiers and types of issues helped the BNE IT team to automatically fix most of the issues, while other less critical issues (e.g., absence of subfields) were assigned to cataloguers to fix them manually.

\subsection{Publication}

The goal of this activity is to make the generated RDF dataset available and discoverable on the Web following the linked data principles and best practices. This activity is decomposed into two tasks: $i$ ) publishing the dataset on the Web; and ii) publishing metadata describing the dataset.

\subsubsection{Publishing the dataset on the Web}

The goal of this task is to make the dataset available following the linked data principles and best practices. The second and third linked data principles stated the following:

"Use HTTP URIs so that people can look up those names" (2)

"When someone looks up a URI, provide useful information, using the standards (RDF)." (3)

The Specification activity described in Section 7.3 already defines that the IRIs assigned to RDF resources should be HTTP IRIs. The goal of the Publication activity tackles the third principle by providing the mechanism for serving RDF data when clients look up these HTTP IRIs. This mechanism is frequently called "dereferenceable URIs". Over 
the years, several technologies have been proposed to provide this mechanism. Examples of these technologies are HTTP servers configured to serve RDF resources ${ }^{18}$, "linked data front-ends" such as Elda ${ }^{19}$ or Pubby ${ }^{20}$, and the more recent W3C Linked Data Platform Recommendation. ${ }^{21}$

As discussed in Section 7.1, the first version of datos.bne.es relied on the "linked data front-end" Pubby to provide "dereferenceable URIs" and content-negotiation in various RDF serializations and HTML. However, since the release of the datos.bne.es 2.0, "dereferenceable URIs" and content-negotiation are provided by the technological stack developed in the thesis to support the datos.bne.es service. We describe in detail this technological stack in Section 7.8.

\subsubsection{Publishing metadata describing the dataset}

In recent years, two major vocabularies have been proposed for describing datasets and catalogs, namely VoID (Vocabulary of Interlinked Datasets) (W3C), and the more recent DCAT (Data Catalog Vocabulary) (Maali et al. [2014]) both published by W3C. VoID focuses exclusively on linked datasets and DCAT provides a wider set of metadata elements for describing datasets and data catalogues (i.e., not neccesarily linked datasets). According to the guidelines provided in the W3C DCAT recommendation (Maali et al. [2014]), DCAT can be used in combination with VoID for describing linked data related aspects such as .

The datos.bne.es dataset is described using a combination of DCAT and VoID and has been published following linked data principles by using content-negotiation under the following IRI: http://datos.bne.es/. If a client requests the Turtle version of the aforementioned IRI, the datos.bne.es server returns an HTTP 303 response with the following location http://datos.bne.es/inicio.ttl, which contains the RDF description of the datos.bne.es dataset using DCAT and VoID. In Listing 7.2, we provide a sample of the metadata description for the current version of the datos.bne.es dataset.

Listing 7.2: Sample of the DCAT/VoID metadata for datos.bne.es dataset

\footnotetext{
${ }^{18} \mathrm{An}$ example is the Apache HTTP Server configured through the .htaccess (see for example https://www.w3.org/TR/swbp-vocab-pub/\#recipe1, Last viewed 04-05-2016)

${ }^{19}$ http: //www. epimorphics.com/web/tools/elda.html (Last viewed 04-05-2016)

${ }^{20}$ http://wifo5-03.informatik.uni-mannheim.de/pubby/ (Last viewed 04-05-2016)

${ }^{21}$ https: //www.w3.org/TR/ldp/ (Last viewed 04-05-2016)
} 
\# see the full file at http://datos.bne.es/inicio.ttl

aprefix datosbne: <http://datos.bne.es/resource/> .

aprefix rdf: <http://www.w3.org/1999/02/22-rdf-syntax-ns\#> .

aprefix rdfs: <http://www.w3.org/2000/01/rdf-schema\#> .

aprefix owl: <http://www.w3.org/2002/07/owl\#> .

aprefix dcat: <http://www.w3.org/ns/dcat\#> .

aprefix void: <http://rdfs.org/ns/void\#> .

aprefix dct: <http://purl.org/dc/terms/> .

aprefix foaf: <http://xmlns.com/foaf/0.1/> .

aprefix rdflicense: <http://purl.org/NET/rdflicense/> .

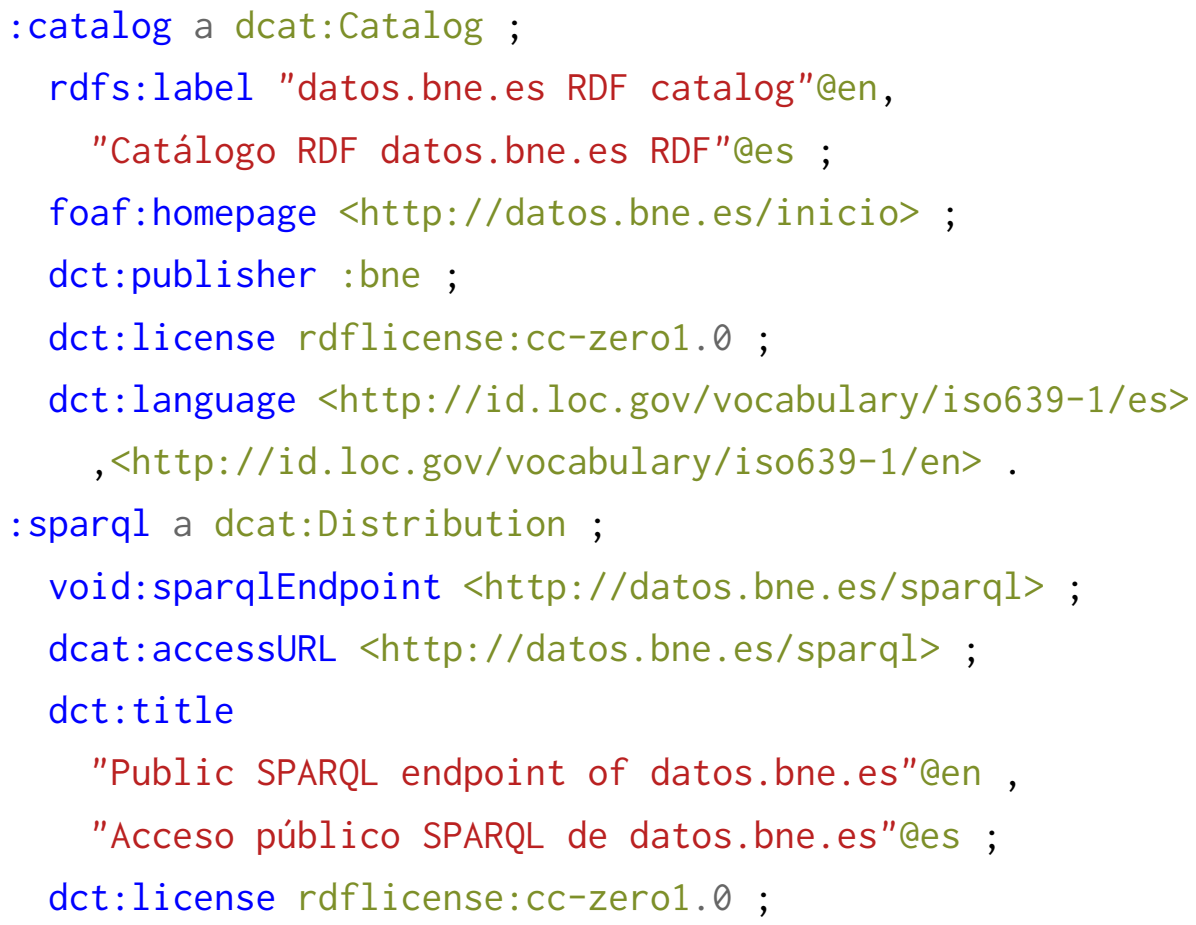

Regarding the multilingual nature of datasets, the DCAT vocabulary provides the ability of annotating the language of the dataset or catalog being described. In Listing 7.2, we show how to include the language following the recommendation provided by the documentation of DCAT. In particular, the DCAT recommendation states that a data publisher should use the URIs maintained by the Library of Congress ${ }^{22}$ in the following way: $i$ ) if an ISO 639-1 (two-letter) code is defined for language, then its

\footnotetext{
${ }^{22}$ The Library of Congress is responsible for maintaining the URIs for the ISO language codes (see http://id. loc.gov/vocabulary/ Last viewed 04-05-2016)
} 
corresponding IRI should be used; $i i$ ) otherwise, if no ISO 639-1 code is defined, then the IRI corresponding to the ISO 639-2 (three-letter) code should be used. In the example of Listing 7.2, we show the annotation corresponding to Spanish and English. The complete file contains the annotation of the 195 languages that are used in the datos.bne.es dataset.

Another important aspect when describing a linked dataset is the license of the data. In DCAT and VoID, the license can be defined using the dct : license property. Ideally, the value of this property should contain a IRI of a machine-readable description of the license in RDF. A valuable resource to find machine-readable descriptions of licenses is the RDFLicense dataset (Rodríguez Doncel et al. [2014]). In Listing 7.2, we show how to define the Creative Commons CCO license for the datos.bne.es dataset.

\subsection{Exploitation: datos.bne.es service}

The goal of this activity is to design and develop applications that use the linked dataset that has been produced and published as a result of the previous activities. Linked data-based applications and user interfaces bring the opportunity of exploring new mechanisms for information navigation, retrieval and visualization. In this section, we introduce the service that we have developed for the datos.bne.es dataset. First, we introduce the main data format used accross the service. Second, we present an overview of its technological architecture. Third, we discuss the main features of the the search engine built on top of the linked dataset. Finally, we give an overview of the main features of the user interface for the service.

\subsubsection{Data format: JSON-LD}

A core design feature of the datos.bne.es service is that it processes, indexes, and handles data directly using the JSON-LD serialization of RDF. This design feature simplifies the data processing and storage across the different layers by avoiding complex data transformations to other data formats. In Listing 7.3, we show the JSON-LD document corresponding the RDF resource of "Miguel de Cervantes Saavedra".

Listing 7.3: JSON-LD document for Miguel de Cervantes

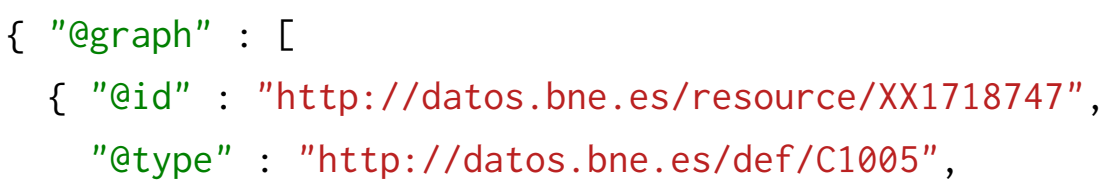




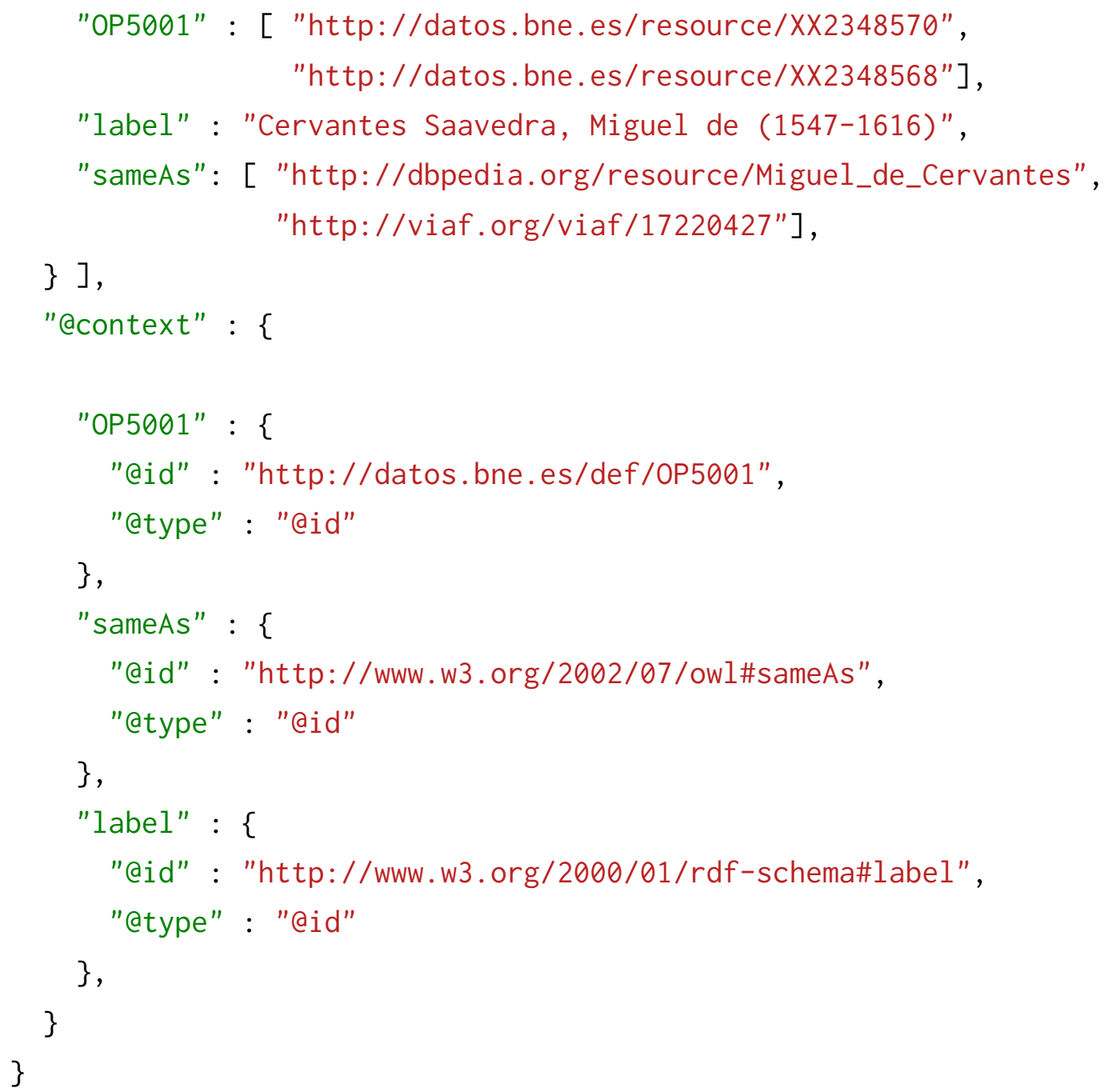

\subsubsection{Architecture}

In this section, we provide an overview of the architecture supporting the datos.bne.es service. As shown in Figure 7.2, the architecture is organized into three layers (from the bottom to the top of the figure): $i$ ) the data layer; $i i)$ the application layer; and, iii) the access layer.

\section{Data layer}

The data layer is in charge of the storage, management and access to the datos.bne.es data. As shown in Figure 7.2, this layer is composed of four components: $i$ ) the indexing and ranking module; $i$ ) the search engine; $i i i)$ the document database; and, $i v$ ) the RDF store. 


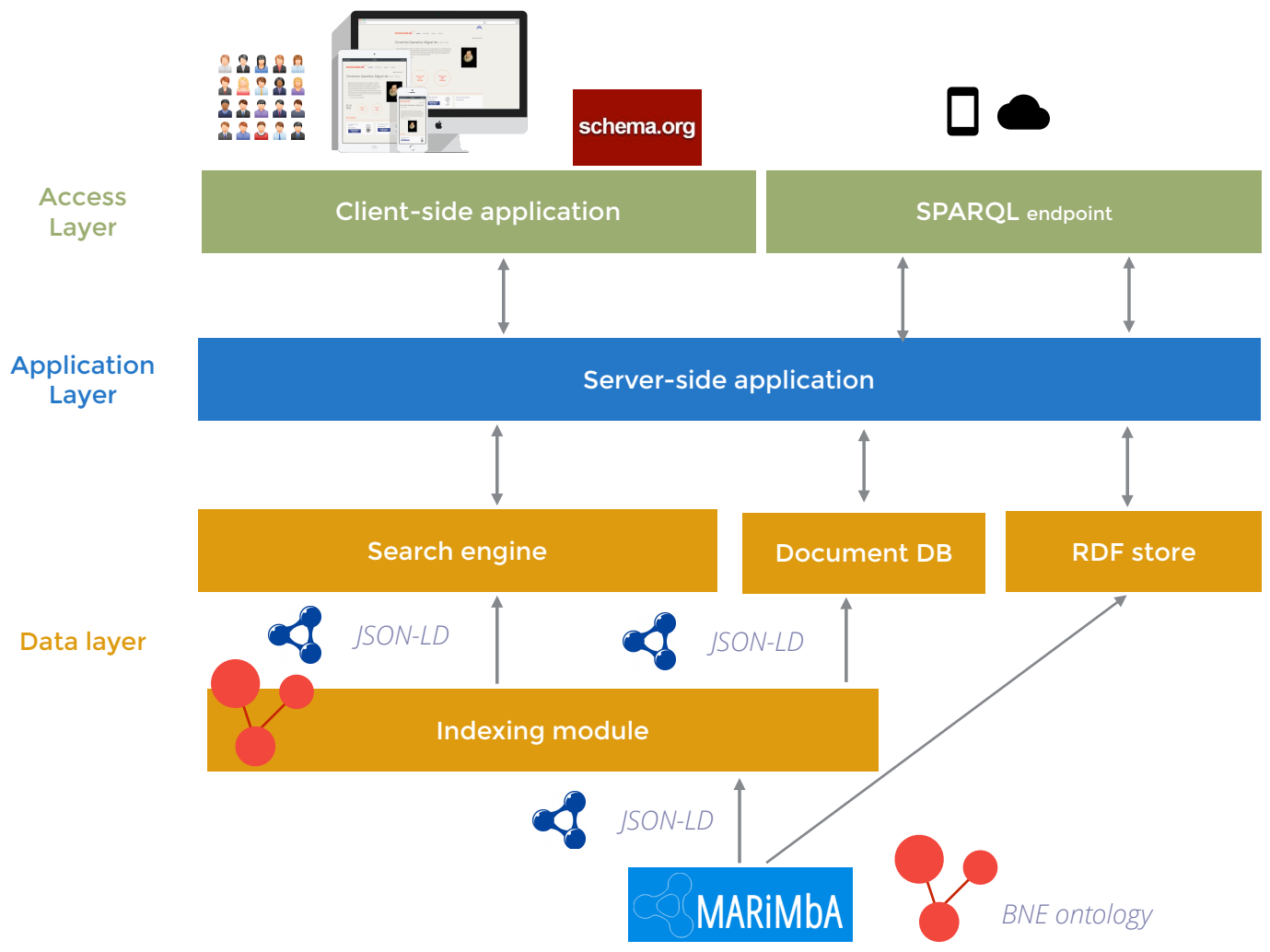

Figure 7.2: Architecture of the datos.bne.es service.

Indexing module. This module processes and indexes the JSON-LD documents, produced by the marimba-tool, into the search engine and the document database. The most important task performed by this module is to assign a weight to each RDF resource. This weight will be used for ranking and presentation in the upper-layers of the architecture. In order to assign a weight to each RDF resource $r$, this modules uses a weighting method $W$, that is described in Algorithm 6. The main idea of the method is to use the outgoing links of an RDF resource to weight its importance in the dataset. These outgoing links are typed according to the object properties defined in the BNE ontology for each type of resource; for instance, the property is creator of (bne:OP5001) for Persons (bne:C1005)). The indexing module allows the dataset maintainers to manually assign the weights assigned to each object property. For example, based on the notion that the higher the number of works created by an author, the more relevant an author is, the weight assigned to the property is creator of (bne:OP5001) would be higher than the weight assigned to other object properties (e.g., owl : sameAs). This type of ranking falls into the category of local link-based rank- 
ing (Baeza-Yates et al. [1999]) with the particularity of using typed links with different weights as opposed to classical link-based ranking where there is only one type of link. Local linked-based ranking produces a ranking based exclusively on direct links between the resources, as opposed to global approaches such as PageRank (Page et al. [1997]), which uses the overall link structure of the dataset to calculate the weigths.

As defined in Algorithm 6, the weighting method $W$ receives two inputs: the $\mathrm{RDF}$ resource $r$ and the weights configuration file $c$. The weighting method proceeds as follows:

- First, the final weight $w$ is initialized to 0 (Line 1).

- Second, the function getOutgoingLinks extracts the number of outogoing links for each object property in $r$ (Line 2).

- Third, for each pair of object property op and number of links $n$ (Line 3):

- The method getWeightForOp retrieves the weight $w_{o p}$ assigned to op in the configuration file $c$ (Line 4).

- Adds the result of $w_{o p} \times n$ to the final weight (Line 5).

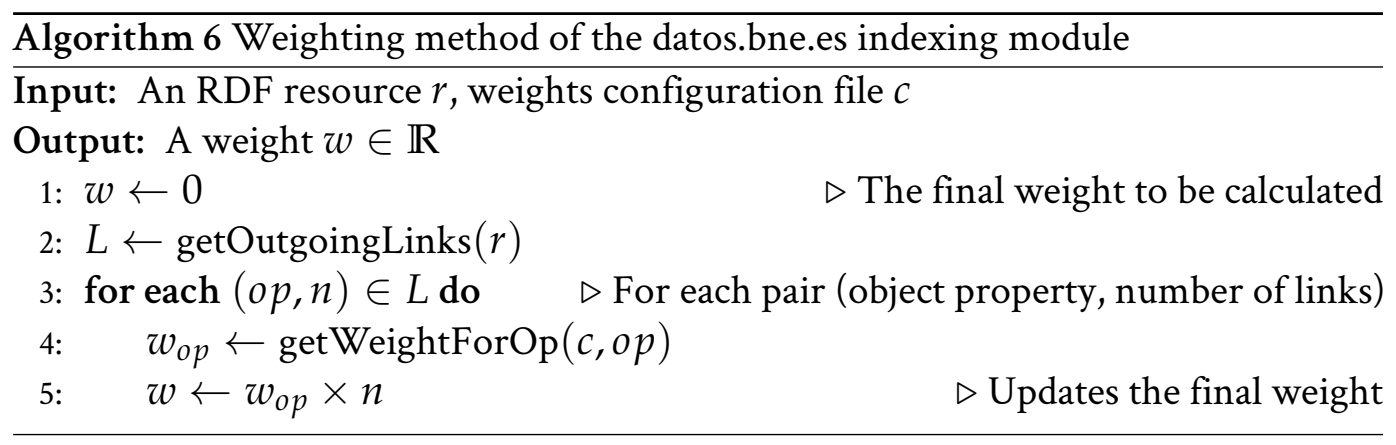

Search engine. The search engine component provides full-text and faceted search capabilities to the datos.bne.es service. The current version of the search engine is based on Elasticsearch ${ }^{23}$, a widely-used search software built on top of Apache Lucene $e^{24}$. One of the most interesting features of Elasticsearch for datos.bne.es lies in the ability to directly index JSON-LD documents, which highly reduces the overhead of preprocessing the RDF documents. The facets provided by the search engine and interface are directly mapped to the main classes of the BNE ontology (e.g., Work, Person).

\footnotetext{
${ }^{23}$ https://www.elastic.co/products/elasticsearch (Last viewed 5th May 2016)

${ }^{24}$ https: //lucene. apache.org/core/ (Last viewed 5th May 2016)
} 
In this way, the user can filter the results by the different types of entity in the dataset. Moreover, in query time, the search engine calculates a ranking score using the weight $w$ assigned to each resource in linear combination with the classical information retrieval score provided by $t f-i d f$. In this way, the search engine provides a search experience which is more oriented to entities than to keyword-based results. Figure 7.3 shows a page with results from the datos.bne.es search interface. The results correspond to the user query "Aristoteles". ${ }^{25}$ In the figure, it can be seen the use of facets by type of entity corresponding to the core classes of the BNE ontology: Obras (Works), Personas (Persons), Entidades (Corporate bodies), and Ediciones (Editions). Moreover, the results are ordered by relevance using the aforementioned ranking score. An interesting feature is that if a certain result is over a certain threshold (empirically configured in the current version), that result is highlighted and a summary of the main connections of the resource are shown (i.e., Aristotle in the left-hand side of the figure). Moreover, as it can be seen in the figure, the keyword "Aristotle" gathered the works of Aristotle among the best ranked results, showing the entity-centric approach of the search engine in datos.bne.es.

Document database. The document database component directly stores and manages the JSON-LD documents generated by the marimba-tool. The document database is the main mechanism used by the applicationg layer for querying and retrieving data. The current version of the datos.bne.es service uses $M o n g o D B^{26}$, a JSON-oriented document database. The main motivation for using a document database, instead of a more sophisticated graph or RDF database, is that the application layer of the service does not need to execute complex queries. For more sophisticated queries, we use an $\mathrm{RDF}$ store and offer a public SPARQL endpoint that is described below.

RDF store. The RDF store component stores RDF data and enables the execution of queries in the SPARQL query language. This component is used by the application layer for: $i$ ) serving different RDF serializations using content-negotiation; and, ii) supporting the public SPARQL endpoint, which can be used by third-party applications to query RDF data. The current version of the datos.bne.es service uses Virtuoso Open Source Edition ${ }^{27}$, a widely-deployed RDF storage software supporting large linked data projects such as DBpedia.

\footnotetext{
${ }^{25}$ http://datos.bne.es/find?s=aristoteles (Last viewed 10th May 2016)

${ }^{26}$ https: //www. mongodb.com (Last viewed 5th May 2016)

${ }^{27}$ https://github.com/openlink/virtuoso-opensource (Last viewed 5th May 2016)
} 


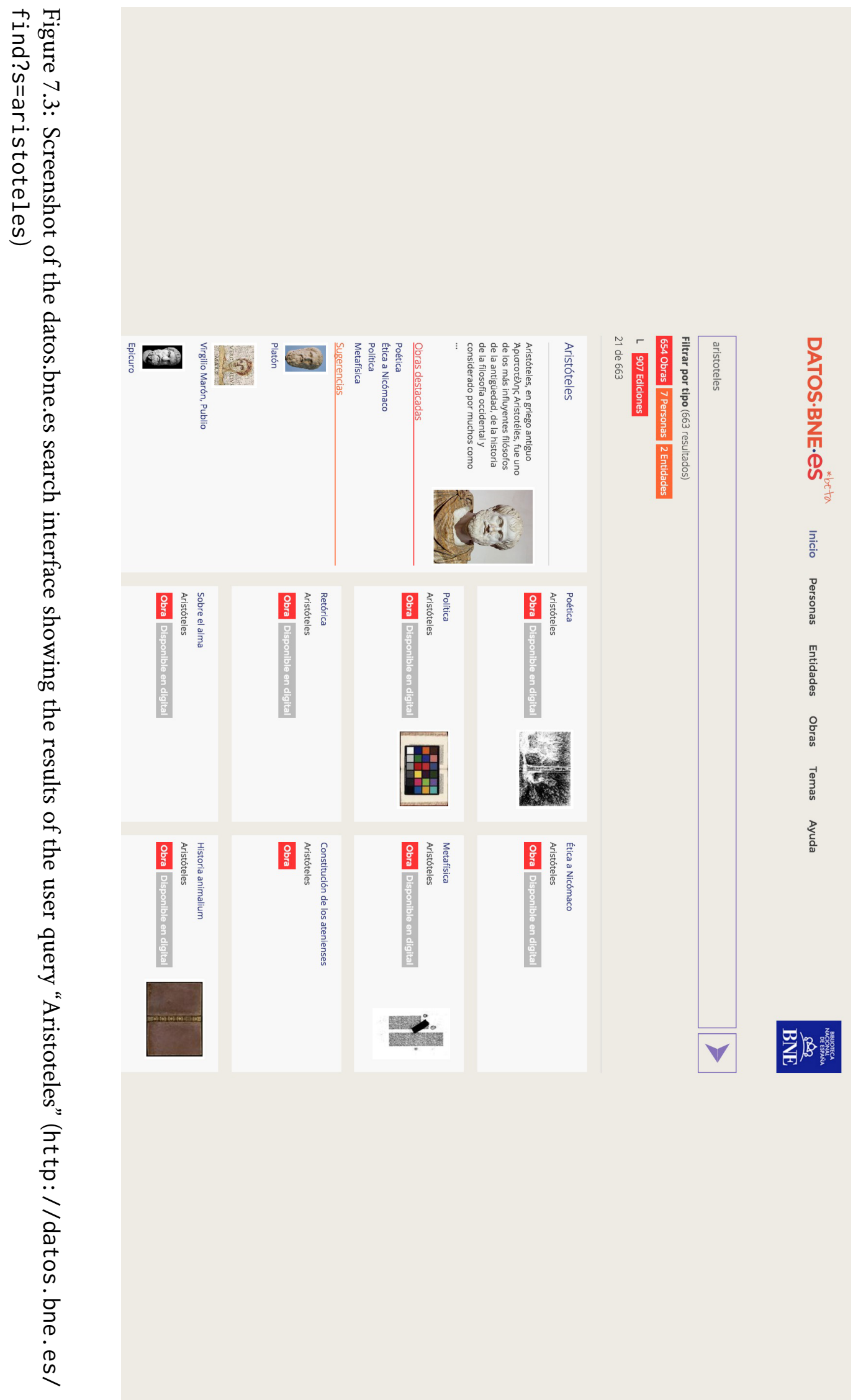




\section{Application layer}

The application layer interacts with the data layer to serve data in different formats, to provide full-text and faceted search capabilities, and to make available the public SPARQL endpoint. The server-side application componenent is completely built in Javascript using node.js. ${ }^{28}$ By using Javascript, node.js provides a natural way of interacting with JSON objects and by extension with JSON-LD objects, which is the core data format used by the datos.bne.es service. The application layer follows the architectural pattern known as "Model-View-Controller" (MVC), where the models abstract the data objects in the data layer, the controllers query and retrieve the models and render different views of the resources. Moreover, the application layer provides a routing mechanism to handle the HTTP requests made to the server. This routing mechanism provides the content-negotiation capabilities of the datos.bne.es service.

\section{Access layer}

This layer provides access to the datos.bne.es data in two different ways: $i$ ) through a User Interface (UI), oriented to end-users; and, $i i)$ through a public SPARQL endpoint oriented to third party applications and developers. Additionally, every RDF resource can be requested in the following RDF serializations: JSON-LD, Turtle and $\mathrm{RDF} / \mathrm{XML}$, using content-negotiation. To this end, the access layer is organized into two components, a client-side application and a SPARQL endpoint.

Client-side application. The client-side application interacts with server-side application to provide an end-user interface, where end-users can search, browse and retrieve information. This application provides a cross-device and cross-browser UI and is developed in HTML5, CSS3, and Javascript. Additionally, the HTML pages generated by the application are annotated with JSON-LD annotations using the schema.org vocabulary. We discuss the UI features in more detail in Section 7.8.3.

SPARQL endpoint. The public SPARQL endpoint of the datos.bne.es service is available at http: //datos. bne.es/sparql. This endpoint enables end-users and applications to issue queries that conform to the W3C SPARQL 1.1 query language.

\footnotetext{
${ }^{28}$ https://nodejs.org/en/ (Last viewed 5th May 2016)
} 


\subsubsection{User interface}

The datos.bne.es user interface allows end-users to search and browse information contained in the datos.bne.es 2.0 dataset. Library catalogues are based on bibliographic records, which are to a great extent both structured and normalized. This characteristic has enabled the use of common "advanced search" options such as filtering and field searching, topic browsing and search using controlled vocabularies. Linked data solutions and UIs can build on these features and bring the opportunity of exploring new mechanisms for information navigation, retrieval and visualization. In particular, the linked data UI of datos.bne.es extensively uses the graph structure of linked data and the BNE ontology to deliver enhanced search, navigation, presentation and access to information. In Chapter 8, we evaluate the impact of this linked data-based UI on end users. The components of the linked data UI can be classified into three categories.

Search (UI-S). The datos.bne.es system follows an entity-centric approach to indexing, ranking and search. The search engine indexes properties describing entities that are modeled according to the BNE ontology (e.g., Persons, Works, Concepts). Ranking and relevance of results are based on weighted links across entities, and qualified by the object properties in the ontology.

Navigation (UI-N). Information navigation is enabled exclusively by following qualified links from one instance to the other (e.g., from an author to work, to the subject of a work). Each page describes an instance of the ontology and it is linked to other instances via its object properties.

Presentation (UI-P). The presentation of information is also derived directly from the ontology, in the sense that the main information blocks of each entity page and their order are determined by the semantic model (e.g., the author pages holds three major blocks defined by the most relevant object properties: e.g., creator of).

\subsection{Summary}

In this chapter, we have provided a brief overview of the application of the contributions of this thesis in the context of a large project, datos.bne.es. As we have shown throughout the chapter, we have proposed and followed a methodology that leverages the best practices available for linked data, and web standards. Moreover, we have shown how scalable and sophisticated services for the library domain can be built by using semantic technologies and the marimba-framework, which we evaluate in the next chapter. 


\section{User-centered evaluation}

The goal of this chapter is to study and evaluate a variety of aspects related to information retrieval, navigation of library content based on ontologies, and user experience, in a controlled but realistic scenario using the datos.bne.es service presented in Chapter 7. This study tackles our third research problem P3: Understanding the impact of ontology-based library data access in end-user applications. To approach this problem we compare two online services: $i$ ) the Online Public Access Catalogue (OPAC), and ii) the linked data-based service datos.bne.es of the National Library of Spain (BNE), described in Chapter 7. The OPAC service is the main entry point to the library collections and has a large user base. The OPAC and datos.bne.es services are of special relevance due to the social impact of the data publisher, the size, diversity and cultural significance of the collections. Moreover, the comparison of these two services is motivated by the following characteristics:

- They enable access to the same underlying large catalogue data composed by more than 4 million MARC 21 bibliographic records, more than 3 million MARC 21 authority records (e.g., providing personal information, classification information), and to more than 100,000 digitized materials. In other words, the two systems manage, process and make available the same amount and type of information.

- They provide comparable functionalities including a search engine over different types of entities (e.g., authors, works, translated works, or subjects), navigation across different types of connected entities (such as the author of a work). Yet, they take a completely different approach to information navigation, visualization and retrieval. 
- The OPAC service is a widely deployed, established and standard system to provide online access to library catalogues and represents the current practice within the library domain. ${ }^{1}$ The datos.bne.es system, as we described in Chapter 7 , extensively applies semantic and linked data technologies and is comparable to other relevant systems, such as the data.bnf.fr service, both in its scale and applied semantic techniques.

The rest of the chapter is organized as follows. First, in Section 8.1, we present the main objectives of the chapter and introduce the two experiments carried out for the evaluation of our fifth hypothesis. Second, we describe and discuss the first experiment, a task-based experiment with 72 students of "library and information science" (Section 8.2). Third, we describe and discuss the usability and user satisfaction experiment carried out with 36 "library and information science" students (Section 8.3). Finally, in Section 8.4 we discuss and summarize our main experimental results and findings.

\subsection{Evaluation objectives}

The main objective of this chapter is the evaluation of our fifth hypothesis that we formulated as follows: "The application of semantic technologies to end-user library application results in significantly better user satisfaction and efficiency to complete information retrieval tasks". In order to evaluate this core hypothesis, we subdivide it into a set of three hypotheses:

H5a. A user finds and retrieves the required information significantly faster with the datos.bne.es system than with the traditional OPAC system.

H5b. A user finds and retrieves the required information visiting significantly less pages with the datos.bne.es system than with the OPAC system.

H5c. The user perception of the system usability, and its satisfaction concerning the user interface is significantly better with the datos.bne.es system than with the OPAC system.

The aforementioned hypotheses share the underlying idea that semantic technologies applied to library catalogues impact positively on users in order to satisfy their information needs. In order to evaluate these hypotheses, we designed two experiments: $i$ ) a controlled user-centered experiment, described in Section 8.2, to evaluate

\footnotetext{
${ }^{1}$ The OPAC used by BNE is powered by a commercial software called Symphony, which is used by more than 20,000 libraries (http: //www.sirsidynix.com/products/symphony). (Last viewed 10th May 2016)
} 
hypotheses $\mathrm{H} 5 \mathrm{a}, \mathrm{H} 5 \mathrm{~b}$, and $\mathrm{H} 5 \mathrm{c}$; and $\mathrm{ii}$ ) a usability and user satisfaction evaluation, described in Section 8.3, to further evaluate hypothesis $\mathrm{H} 5 \mathrm{c}$.

\subsection{Experiment 1. Task-based experiment}

In order to examine the three hypotheses defined in Section 8.1, we conducted a usercentered experiment. The experiment was conducted in a controlled computer room with students of the Library and Information Science School at "Universidad Complutense de Madrid" in four sessions during May 2015. The details of the experimental settings are explained in the following sections.

\subsubsection{Experimental settings}

The main goal of the experiment was to collect measures to test our hypotheses by comparing two services: the OPAC service, and the datos.bne.es service. We describe the evaluation measures and the application to collect them in Section 8.2.2. In order to compare the two systems, the experiment was designed as a task-based, betweengroup experiment with two different user groups. In the experiments, all participants worked with the same technical setting and in the same computer room where all computers had similar technical specifications. In each session, we splitted the participants into the following two user groups:

OPAC user group. The first user group carried out the experiment using the OPAC system available at http: //catalogo.bne.es.

datosbne user group. The second user group carried out the experiment using the datos.bne.es system available at http://datos. bne.es.

In the experiment, each subject was asked to complete a scenario of ten tasks that involved interacting with one of the two systems to search, browse and select information. We describe this scenario and its design in Section 8.2.5. It is worth noting that: i) every subject only participated once; $i$ ) every subject used exclusively one system, iii) the task-based scenario was the same for every participant and user group, and iv) the subjects did not know in advance which system they would be using for the experiment. In Section 8.2.4, we provide details about the statistical characteristics of the subjects of our study. 


\subsubsection{Evaluation measures}

In the experiment, we measured three variables (two quantitative variables and one subjective variable). These variables were registered for every task and for every participant in the study. We provide a definition of each of these variables below.

Definition 8.2.1 (Task completion time) The task completion time measure (TCT) is the time that goes from the start until the completion of a retrieval task.

Definition 8.2.2 (Task total pages visited) The task total pages visited measure (TPV) is the total number of pages visited from the start until the completion of a retrieval task.

The task completion measure is a widely-used variable for measuring the efficiency and perceived user satisfaction of interactive information retrieval systems and web search systems (Kelly [2009], Xu and Mease [2009]). Additionally, the total number of pages measure provides a measure of the efficiency of a search and retrieval system in satisfying the information needs of a user and complements the TCT measure. The TCT and TPV provide an indicator of the efficiency and user satisfaction offered by a system and are critical to succesful information retrieval systems (Su [1992]). However, several authors have argued that there are other factors that greatly influence the perception of the user about a system, such as "task difficulty" or "usage proficiency" (Cheng et al. [2010]). In order to account for this limitation, in our study we used a subjective measures that is provided by each user after completing a task. Specifically, after each task the user was presented with a "subjective assesment" questionnaire to register the "task user satisfaction" that is defined as follows:

Definition 8.2.3 (Task user satisfaction) The task user satisfaction measures (TUS) quantifies the user satisfaction of the user with respect to an specific task, after its completion. This measure is provided by the participant using a Likert-scale from 1 to 5 (very satisfactory to very unsatisfactory).

The TUS measure is calculated using the standard System Usability Scale (SUS) (Brooke $[1996,2013])$, in which the system with the highest average score is the most satisfactory. To this end, the 1-5 score is rescaled (specifically: $1 \rightarrow 10,2 \rightarrow 7.5$, $3 \rightarrow 5,4 \rightarrow 2.5,5 \rightarrow 0$ ).

The above-described measures were collected during the experiments using an online application, which we describe in Section 8.2.3. 


\subsubsection{Online application and data collection}

The experiment was conducted using an online application, specifically developed for the experiment. The workflow of the experiment is composed of three steps:

1. Context of use questions. In order to understand the characteristics of each participant, the application asks the participant to fill a form. Specifically, we focused on personal attributes (age and academic year), and experience, knowledge and skills (previous experience with the OPAC and datos.bne.es systems). The results obtained are described in Section 8.2.4.

2. Introduction to the scenario. The application provides an introduction to the scenario by providing a detailed description. The overall scenario is described with special attention to aspects that connect it to a real setting. We present the scenario in more detail in Section 8.2.5.

3. Tasks. The application provides the participant with a set of ten consecutive tasks. Each task is composed by a title and a description. For instance, the user is asked: Find and retrieve the title of two works written by Santiago Ramón y Cajal. The maximum time to complete each task is ten minutes. Once the participant reads the description, (s)he is presented with a time counter and the system screen to start the task. Once (s)he completes the task, (s)he is presented with a form to answer the question and a brief questionnaire for the subjective assessment. Once the user finishes the task (s)he must press a stop button. We present the tasks in more detail in Section 8.2.5.

\subsubsection{Subjects}

In this section, we present the statistics of the population of our study, based on the data collected from the use of context questionnaire described in Section 8.2.3. We performed a between-group study with 72 participants distributed into two user groups (UG) of 36 participants each. In the questionnaire, we collected two personal attributes (age and course) of each participant, and two attribute related to experience and skills with the systems under study (OPAC and datos.bne.es). In order to establish the experience, the participants provided a range of times they had previously used the systems with the following 5-point scale: 0, 1-10, 20-40, 40-60, more than 60 times.

The age of the 72 participants ranged from 18 to 58 years with an average of 26.98 years ( $s d=9.94$ years). The age of participants within the OPAC UG ranged from 18 
to 56 years and averaged 27.47 years ( $\mathrm{sd}=9.67$ years). Within the datosbne UG the average age was 26.5 years ( $\mathrm{sd}=10.32$ years).

Regarding the academic year, we ran the experiment with undergraduate students in their first (19 participants), third (28 participants), and fourth year (14 participants), as well as postgraduate students (11 participants). Within the OPAC UG, 10 participants were in their first year, 12 in their third year, 8 in their fourth year and 6 were postgraduate students. Within the datosbne UG, 9 participants were in their first year, 16 in their third year, 6 in their fourth year and 5 were postgraduate students.

Concerning their previous experience, only $12.5 \%$ of participants had never used the OPAC system, approximately $15.3 \%$ had used it more than 60 times, $26.4 \%$ more than 40 times, and $52.8 \%$ less than 20 times. The datos.bne.es was less frequently known, with approximately $28 \%$ of participants that had never used it, and only $3.6 \%$ that had used it more than 20 times. Within the OPAC UG, 19.4\% of participants had previously used the OPAC system more than 60 times, 38.8\% more than 20 times, and $47,2 \%$ between 1 and 20 times. Within the datosbne UG, 88,9\% of participants had used the system less than 10 times, and the rest $11,1 \%$ between 10 and 40 times. These data highlight two important aspects: $i$ ) almost half of the users of the OPAC UG were relatively profficient with the OPAC system, and $i i$ ) almost all users of the datosbne UG had very little experience with the datos.bne.es service.

\subsubsection{Scenario and tasks}

In order to perform our evaluation, we designed a realistic scenario composed of ten related and consecutive tasks. This scenario is related to the areas of documentation, journalism, digital humanities, and information sciences. The participants in the study, students in these areas, were already familiar with this kind of information retrieval tasks. The scenario was carefully designed together with professors of Library and Information Sciences departments and tailored to the background of the expected participants. Nevertheless, as we introduced in Section 8.2.4, we performed a context of use analysis in order to assess the impact of experience and skills of the participants in the results of the study.

From the point of view of the user, the scenario presents an overall objective: to collect information related to Santiago Ramón y Cajal, one of the most important Spanish scientists of the last century, for the documentation department of a media company that is preparing an article about the author. This main objective is divided into ten consecutive and descriptive tasks with different levels of complexity. 


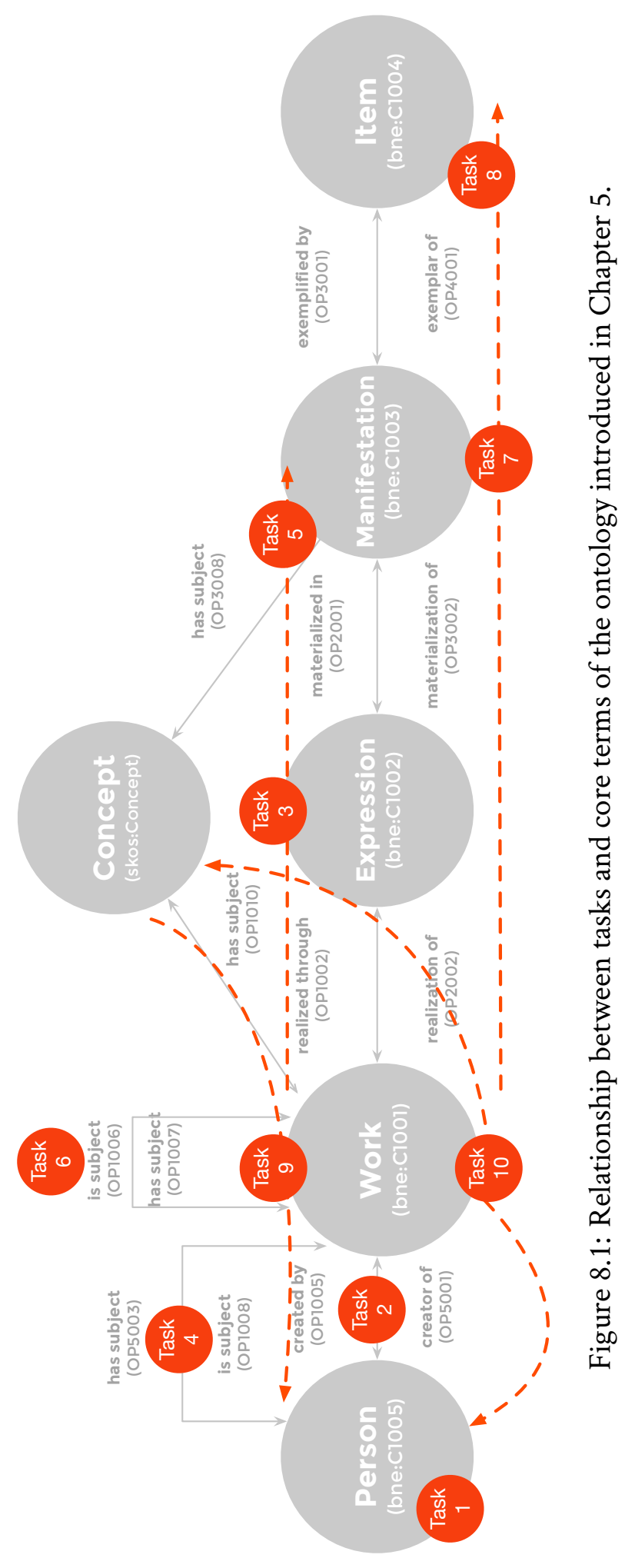


According to Ingwersen and Jarvelin [2005], there is a need for "a further development of the cognitive viewpoint for information seeking and retrieval by providing a contextual holistic perspective”. User-centered approaches offer several advantages, such as simulated work task situations, which might trigger the simulated information needs in "the platform against which situational relevance (usefulness) is assesed" Ingwersen and Jarvelin [2005]. Our experiment described the information need and its context, the problem to be solved and specifically intended to provide to the user a way to understand the goals of the experiment.

With regard to the complexity of individual tasks, it is worth noting that the main aim of our user-centered experiment was the evaluation of a full and realistic exercise, therefore there are several tasks that can be considered easy to solve with both systems (such as retrieving biographical information of an author), and other more complex tasks (such as finding the subject of a certain edition). Thus, our main hypotheses are related to the full scenario composed of interrelated tasks, rather than to individual evaluation of tasks. Nevertheless, to broaden the scope of the study, we designed the tasks to evaluate key aspects that presumably differentiate the datos.bne.es system from the OPAC system. Furthermore, the experiment was designed to keep a balance between a wide scope of analysis, coverage of features, and maintaining the scenario coherent to the participants.

Each task was designed taking into account two dimensions: $i$ ) the ontology terms; and $i$ ) the UI features. Figure 8.1 shows graphically the relations between tasks and the ontology classes and properties presented in Chapter 5, dashed red arrows indicate the expected path to complete the task, and the position of the red circle indicates from which entity the information is expected to be retrieved. Additionally, the tasks were aligned to the UI features described in Section 7.8 .3 of the previous chapter. Finally, Table 8.1 summarizes the ten tasks, the expected navigation, and the associated UI features. The complete description of the tasks is available online. ${ }^{2}$

\subsubsection{Visualization of the experimental results}

Along this chapter we use visually-rich error bars ${ }^{3}$ to provide a mechanism to understand the data distribution and statistical significance of the results of our study. This technique provides a visual alternative to statistical significance tests such as p-values

\footnotetext{
${ }^{2}$ http://sites.google.com/site/usingdatosbne

${ }^{3} \mathrm{~A}$ further description of this visualization technique is available at http://sites.google.com/ site/lightsabererrorbars/ (Last viewed 10th May 2016)
} 


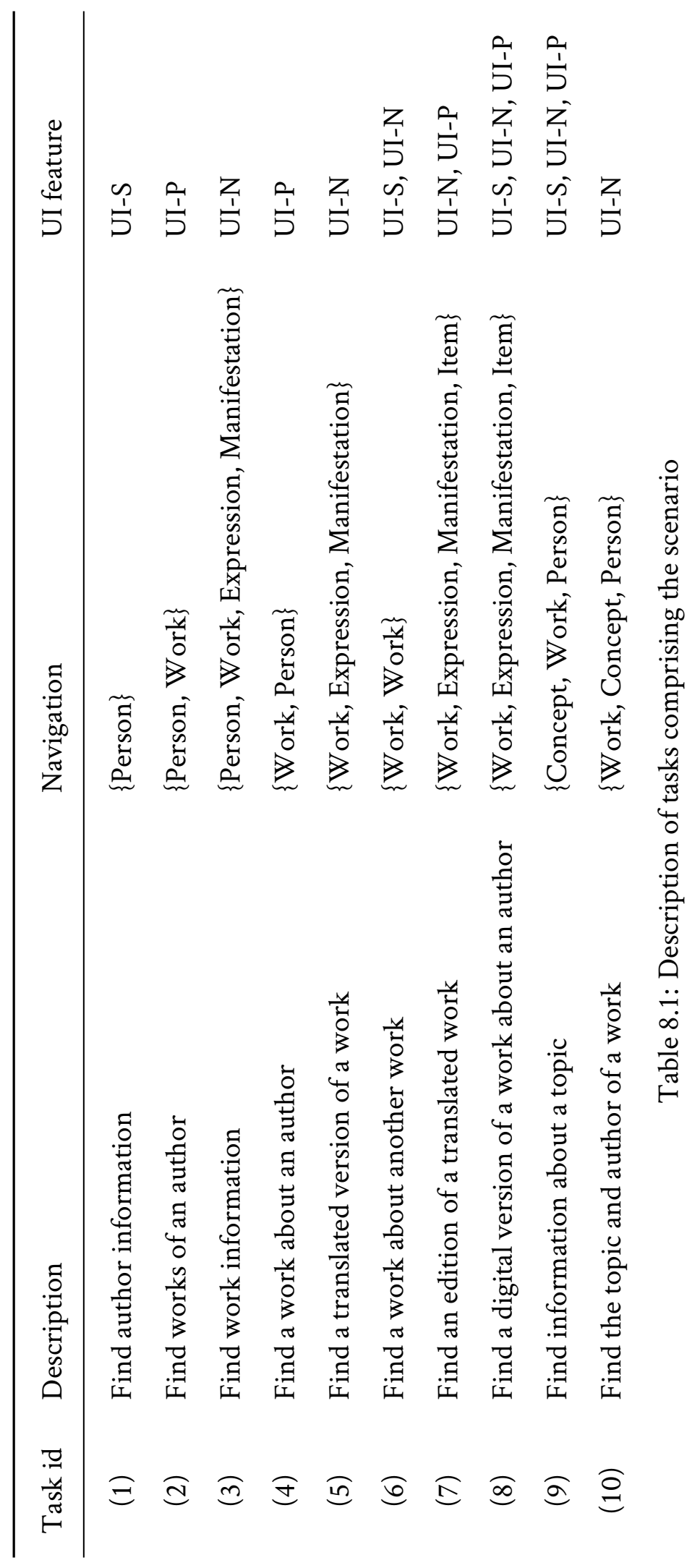


or normality tests. To this end, our graphical representation integrates the following pieces of information:

- The "standard deviation" bar represents the distribution of the data, which we complement with a "violin graph" to provide a graphical representation of the density of data points.

- The "95\% confidence" intervals. The graphical representation of these intervales converge to the mean of the distribution. For a the number of data points that we collect in our study, this graphical representation shows the following properties:

- i) If the intervals of the two data samples do not overlap, there is an evidence of the statistical significance of the results.

- ii) If the intervals of the two data samples overlap up to $50 \%$, there is a 95\% probability that the results are statistically significant.

- The "standard error" bar. If the "standard error" bars overlap, there is a lack of evidence of the statistical significance of the results.

\subsubsection{Experimental results}

In this section, we present the results of our within-group experiment.

\section{Task completion time and visited pages}

Figure 8.2 shows the average time, based on the Task Completion Time (TCT) measure, required by participants to complete all the tasks for both systems. The figure also illustrates the average TCT required per task. The experimental results show that users of the datosbne UG require $32 \%$ less time than users of the OPAC user group, to finish the complete set of tasks. Moreover, for some tasks, such as T5 and T10, the difference is even more significant (50\% less time).

A detailed view of TCT for each participant is shown at the top of Figure 8.3 Each dot represents a participant. The "violin" figure shows the distribution of the responses, and the three error bars (see the description of error bars in Section 8.2.6) show the standard deviation (external thin gray bars), 95\% confidence interval (middle thick gray bar), and standard error (inner thick black bar). The figure clearly shows that there is a statistically significant difference between the systems confirming our first subhypothesis (H5a). 


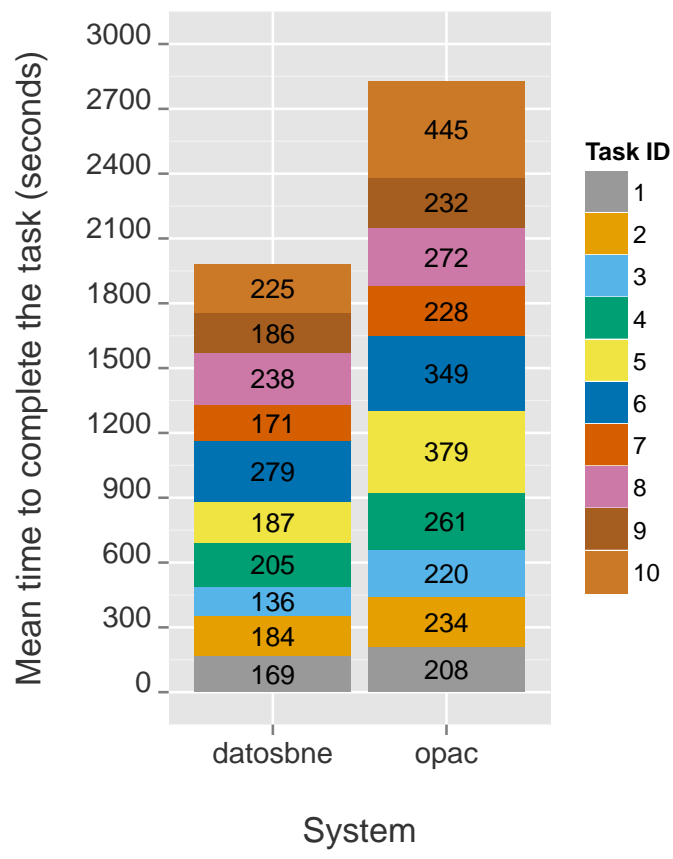

Figure 8.2: Average time to complete all the tasks, showing the average time required for each task.

The bottom part of Figure 8.3 shows the number of pages visited by each user to complete the proposed scenario (an aggregation of the TPV measure for the complete scenario). On average, users of the datosbne UG required to navigate $40 \%$ less pages than users of the OPAC UG to achieve the proposed tasks. The error bars clearly show that there is a statistically significant difference between the systems confirming our second subhypothesis (H5b).

Additionally, we have analyzed the effect of the user's characteristics (e.g. age, course, gender, experience). The most relevant findings are related to age and experience. Figure 8.4 shows the TCT required to complete the proposed scenario faceted by participant age. We aggregated ages in three ranges. It is noticeable that, statistically, there is no significant difference for participants in the ranges (17 to 22 years) and (22 to 30 years). However, people in the higher range (30 to 65 years) showed a significant difference for datosbne. On average, participants with ages in that range (30-65) needed 15\% more time than younger participants (1950 seconds for datosbne users versus 2250 seconds for OPAC user group users).

Concerning the experience of participants, Figure 8.5 shows the time required to complete the proposed scenario, aggregating the participants by the number of times they used the OPAC system. Although we expected that most experienced OPAC UG 

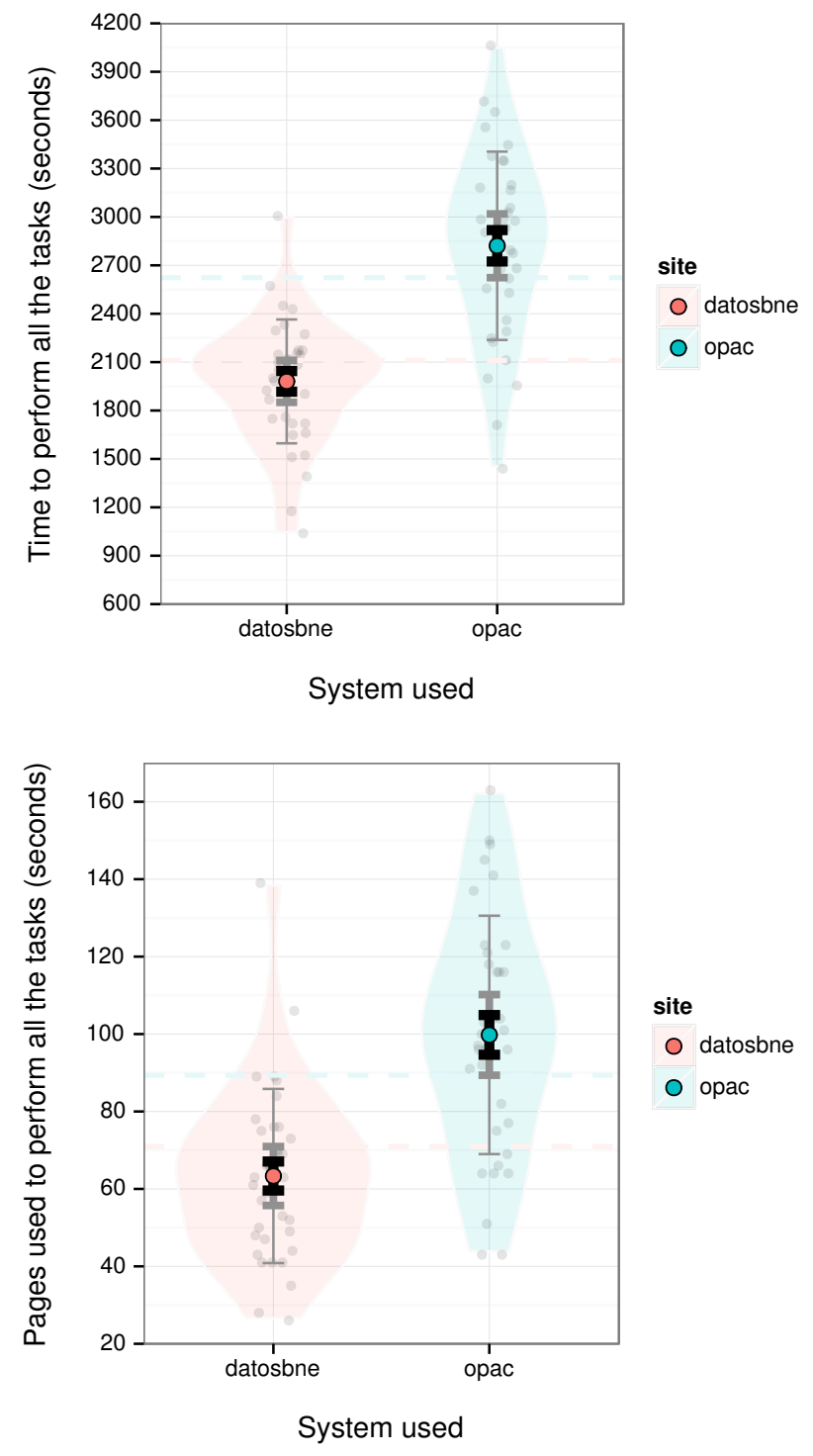

Figure 8.3: Task completion time and visited pages required to complete the tasks proposed to the participants. Each dot represents a user. As the $95 \%$ confidence intervals overlap less than $50 \%$, the differences between the systems are significative and we can reject that differences are due to statistical fluctuations of the samples. 


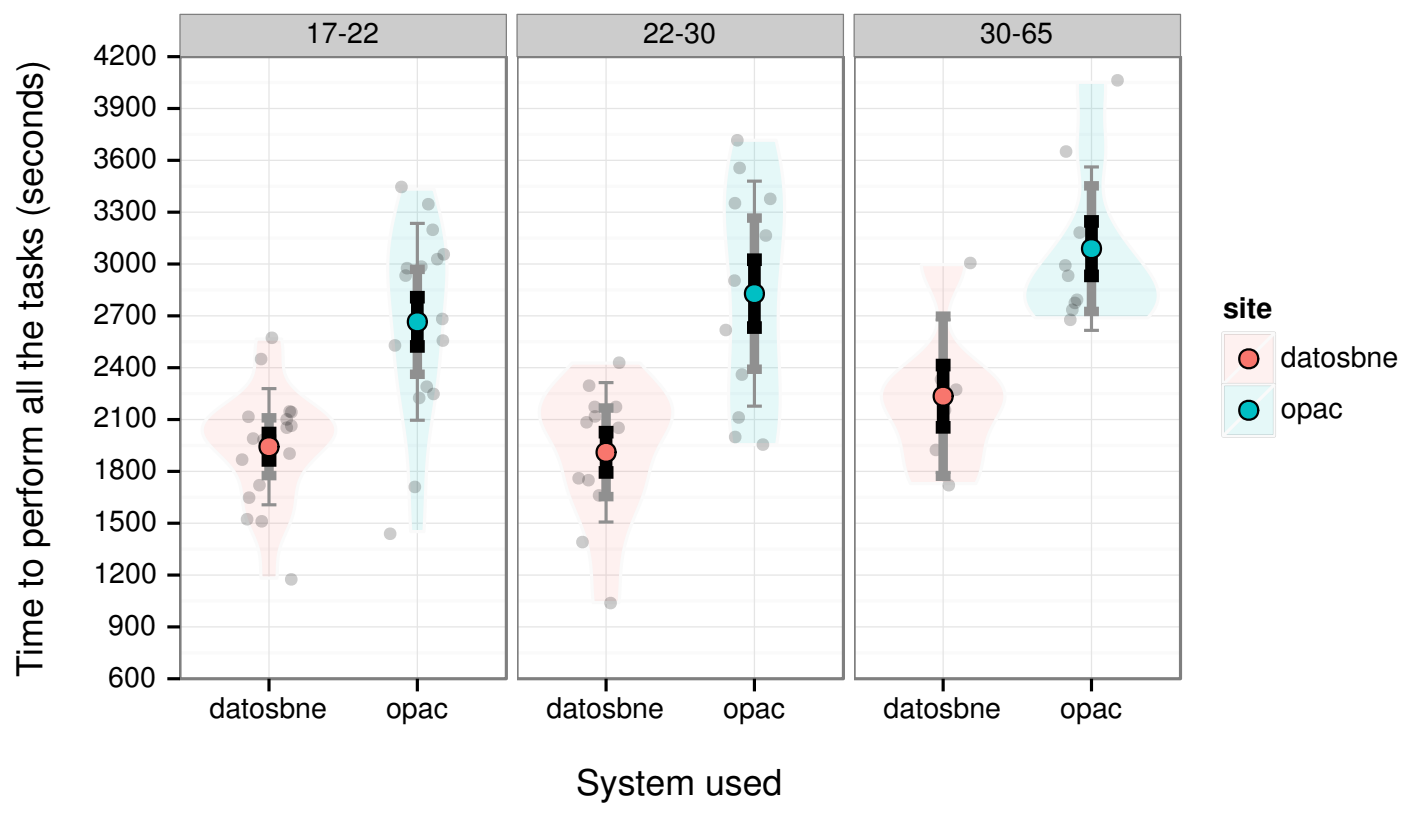

Figure 8.4: Effect of the age. There is a statistical significant increment for datosbne participants in the year range 30-65.

users would require less time to achieve the proposed tasks, we can see that there is no significant differences for any range of usage experience.

Figure 8.6 shows a similar aggregation, but considering the experience with datosbne. Here we also expected better results for participants proficient with the new system and the results are more consistent with the idea that more experienced users will show better task efficiency.

\section{Per-task TCT and TPV results}

In addition to the evaluation of our hypotheses for the complete scenario, in this section we discuss the results by analyzing the effect of datos.bne.es features on each separate task. In particular, we were interested in studying how each task, and the performance of the participants, are related to the semantic modelling (Figure 8.1), and the UI features (Table 8.1). We present the results in Figure 8.7.

From an overall perspective, we observe that datos.bne.es performed better both in task completion time and number of visited pages for every single task. Some differences are more prominent (Tasks 3, 5 and 10), while other less complex tasks like 1 and 2 show an improvement but not highly significant. The fastest task to complete 


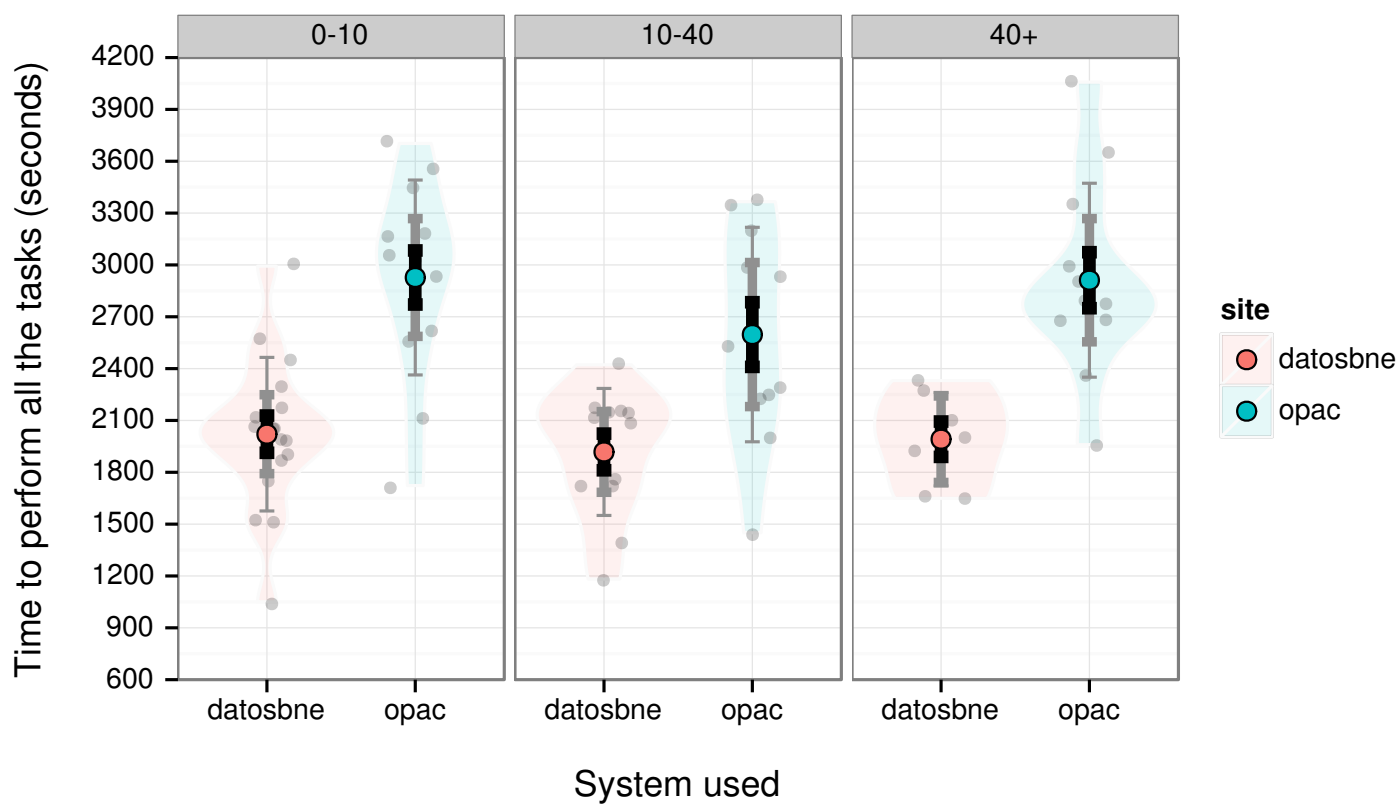

Figure 8.5: Effect of the experience on OPAC. There is no significant relation related to experience on OPAC.
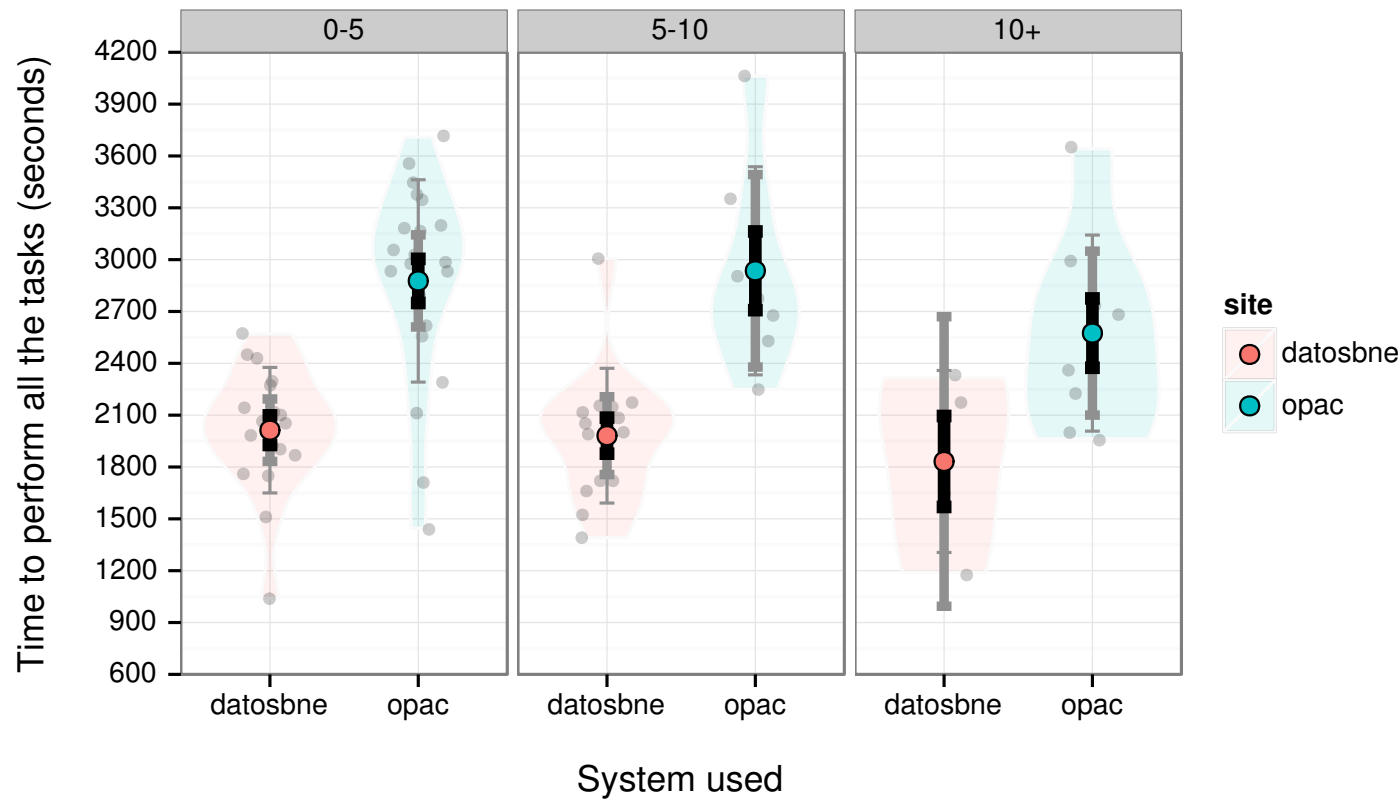

Figure 8.6: Effect of the experience on datos.bne. There is no significant relation related to experience on datos.bne. 
on average with datos.bne.es was task 3 and with OPAC task 1, while the slowest in datos.bne.es was task 6 and with OPAC task 10. Regarding dispersion of the sample for each system, the results by task confirm that the OPAC UG presents a much more dispersed sample of results. However, the results for certain tasks performed by the datosbne $U G$ show a dispersed distribution (especially task 6).

As shown in Figure 8.7, tasks 5 and 10 are significantly faster to perform using the datos.bne.es system. From the point of view of the UI, both tasks have a strong navigational component (UI-N) as they require the user to navigate across several entities. Our results indicate that with this kind of complex navigational tasks users perform significantly better, due to the graph structure and the underlying ontology. Task 3 shows similar characteristics (to a lesser extent) and also has a strong navigational component. With regard to the impact of the semantic modelling, tasks 3 and 5 share a common feature, which is the use of the Work, Expression and Manifestation concepts, where a user starts with a work and navigates to different versions and editions. However, the difference in time between 3 and 5, seems to be the language property (task 5 requires to retrieve a title of a work in English). It is worth noting that finding editions by languages could be reproduced in the OPAC system through the advanced search interface, according to our results, this feature does not seem to have helped users in completing the task.

Finally, in Figure 8.7, the results show that there is not always a correlation between time to complete the task and the number of pages visited. For instance, task 1 and 2 have comparable distributions with regard to time but not with regard to pages.

Regarding the characteristics of users, Figure 8.8 provides a detailed view of the academic course of the participants. The results show that differences between user groups tend to be consistent accross different courses.

\section{User satisfaction}

In order to validate our hypothesis regarding user satisfaction and perception of the compared systems (H5c), we collected user satisfaction after each successfully completed task (TUS). Consequently, we asked the user about the level of satisfaction after completing the task, using a five-point Likert scale (very satisfactory to very unsatisfactory). Figure 8.9 shows the results gathered in the experiment. The figure shows that the datos.bne.es system has an average score of 81 , a score $17 \%$ higher than the OPAC average score (64). The error bars show that this difference is statistically significant, which confirms our third hypothesis ( $\mathrm{H} 5 \mathrm{c})$. 
[Enhancement in time required to achieve the proposed set of tasks]

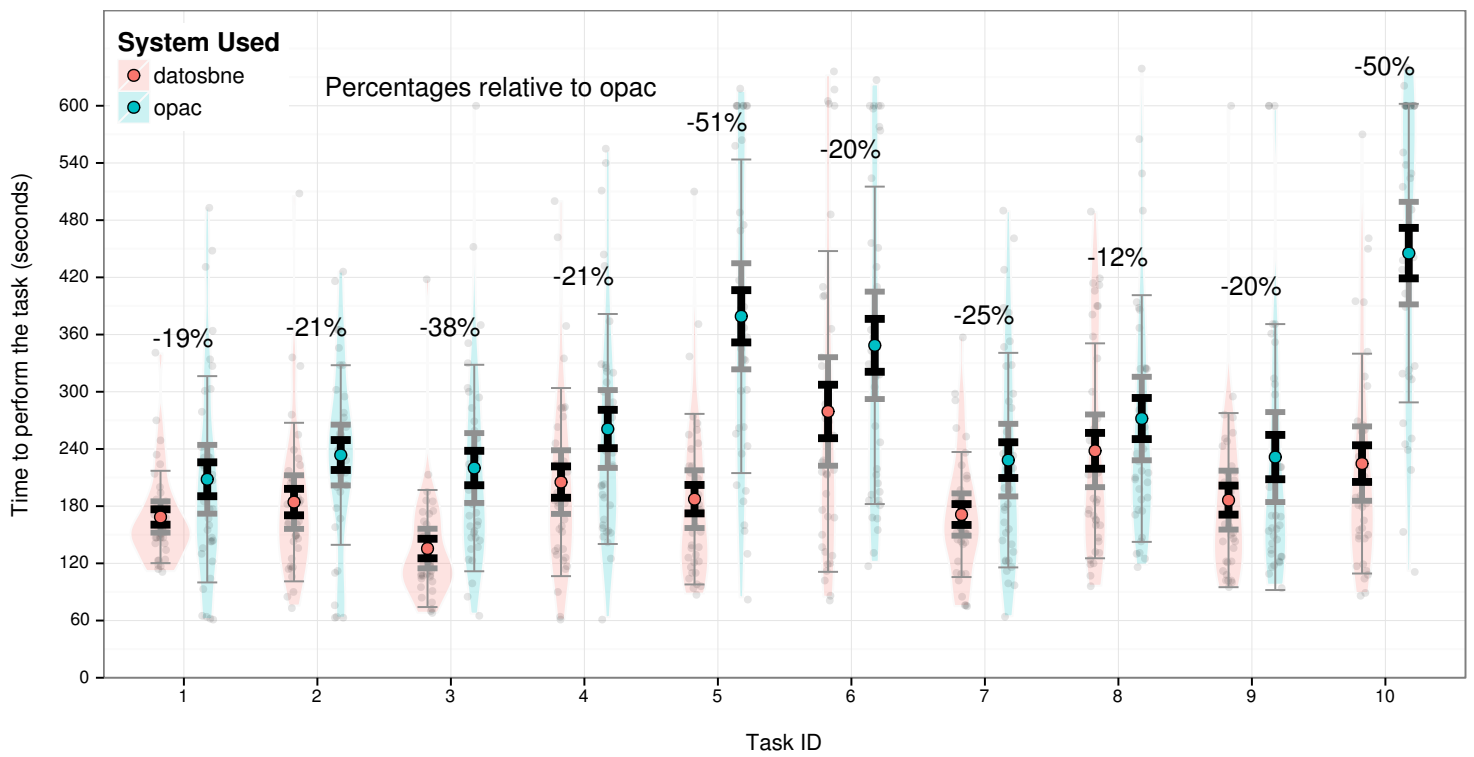

[Enhancement in the number of pages visited to achieve the proposed set of tasks]

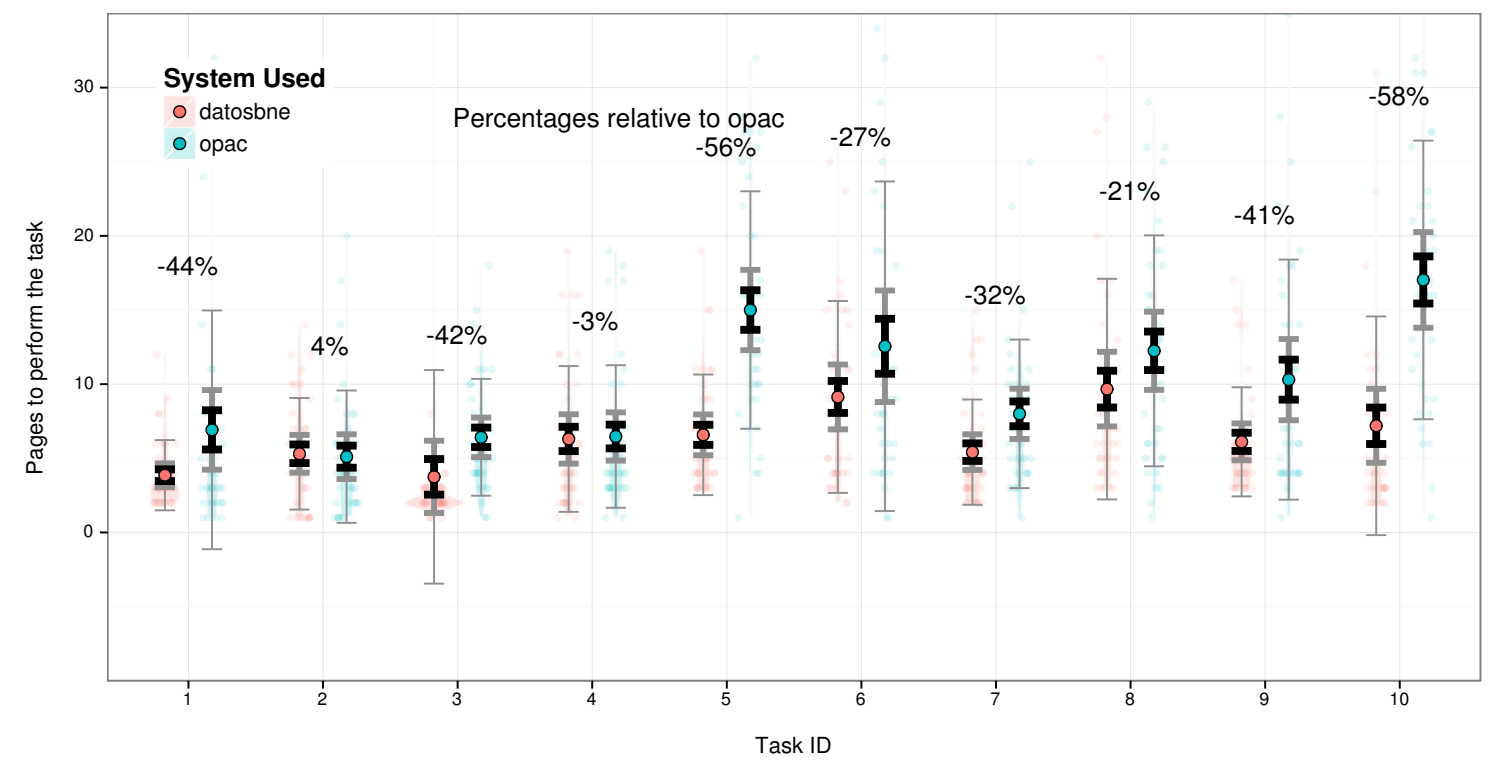

Figure 8.7: Enhancement in task completion time (TCT) required to achieve a task and pages visited (TPV) for the systems compared (OPAC and datos.bne). The error bars correspond to standard deviation (most external), 95\% confidence interval, and standard error (most inner bars). 


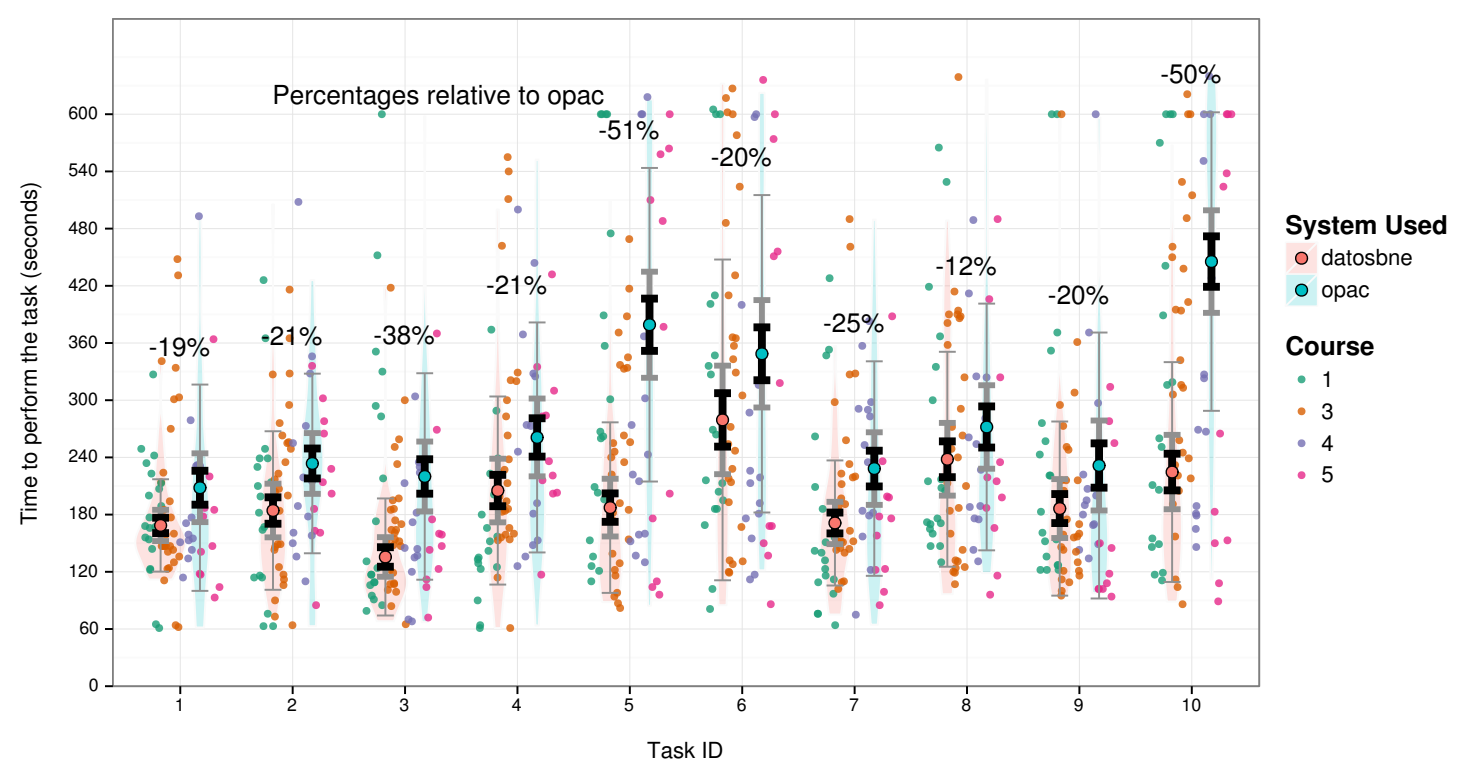

Figure 8.8: Effect of the course. We do not find a significant segmentation by course.

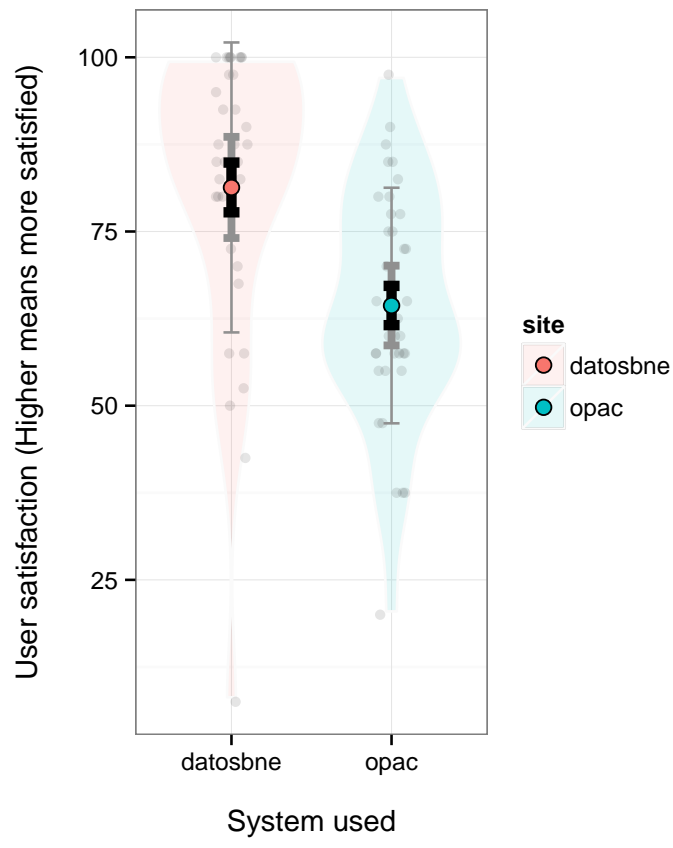

Figure 8.9: Average satisfaction after completing all the tasks, showing the average satisfaction in the range used by the SUS questionnaire. The differences in the average values for both systems are statistically significant. 


\subsection{Experiment 2. Usability and user satisfaction evaluation}

In order to further measure the usability and user satisfaction of the two services, we performed a second experiment with two groups of 18 of students from the Library and Information Science School at "Universidad Complutense de Madrid" during one session in May 2015. The first group evaluated the OPAC system, and the second group evaluated the datos.bne.es service. In order to achieve a $90 \%$ confidence level for a given mean with error lower than $1 \%$, it is required to take 15 measurements at least (Efron and Tibshirani [1986]). This assumes intervals based on a normal population distribution for mean, as we consider in this case. To this end, we ran the experiment with a number of participants slightly superior to 15 participants for each group.

Participants used the system to search, navigate and find information related to the scenario used in the first experiment. In this case, there was no maximum time to finish the use case, most users required around 20 minutes. Once the participant completed the use case, a detailed questionnaire was presented to her or him ${ }^{4}$.

\subsubsection{Methodology and experimental setting}

This questionnaire has 50 questions and 3 free text questions, evaluating two features: i) usability of the online system, and $i i)$ user satisfaction concerning the user interface of the application.

The usability of the system was measured by means of a standard test named "Practical Heuristics for Usability Evaluation” (Perlman [1997]). This test includes 13 questions, in which possible responses must be assigned an integer from 1 (negative evaluation) to 5 (positive evaluation), a Likert scale. Two additional questions were free text questions regarding positive or negative aspects of the evaluated system and its usage. The results of this test are shown in Fig. 8.3.1. This figure shows the average value assigned by the participants to each question, the $95 \%$ confidence error bars, and the standard error bars. The horizontal line is the average value assigned (3.67 to OPAC user group, 3.79 to datosbne) and its deviation bounds (standard deviation is 0.26 for OPAC user group, and 0.25 for datosbne).

In order to evaluate the user's satisfaction, we used a slightly modified version of the standard test "User Interface Satisfaction", proposed by Chin et al. [1988]. The

\footnotetext{
${ }^{4}$ The full questionnaire is available at http://sites.google.com/site/usingdatosbne)
} 
standard version includes 27 questions, but it was reduced to 25 due to overlaps with the usability test described previously. Valid responses to these questions are positive integers ranging from 0 (not satisfied at all) to 7 (completely satisfied).

\subsubsection{Results}

The results for $O P A C$ user group and datosbne are shown in Figure 8.10. The average value for user satisfaction was 5.16 for OPAC user group and 5.48 for datosbne, with a standard deviation of 0.35 and 0.49 respectively.

Figure 8.10 highlights that, on average, datos.bne.es produces better values for both usability and user satisfaction. If we focus on the categories of the questions ( 3 for usability, and 5 for satisfaction), the figure shows, concerning usability, datos.bne.es shows a better evaluation in 4/4 questions of category "Learning", 2/5 questions for "Adapting to the user") and 3/5 questions "Feedback and errors". Concerning user's satisfaction, datos.bne.es has a better evaluation in $5 / 5$ questions in category "Overall", there is no clear benefit in category "Presentation", get a lower evaluation when compared to the OPAC system in category "Information", get better evaluations in 3/6 for category "Learning", and get better evaluation in 5/5 questions for "Capabilities". However, lightsaber error bars show that there is not a statistical evidence to support the better results obtained for datosbne. We cannot reject the possibility of having these results due to a statistical fluke.

The free text questions allows receiving recommendations from participants. Most of these recommendations and suggestions will be implemented in the next version of the application. Most evaluators remark the aforementioned perceived benefits: simplicity, clarity, and intuitiveness. Other users pointed out that they missed an "advanced" search and a clearer navigation accross intermediate search results.

Moreover, we also adapted these data to a 1-7 scale in order to compare with the fine-grained user satisfaction analysis (presented in Section 8.3). Specifically we computed the average satisfaction (mean over each user task) of each user (in the range 1-5), and this value was re-scaled to a 1-7 range. Results are shown in Figure 8.11. It is remarkable that the average values obtained are similar to the ones obtained with the fine-grained questionnaire in its overall facet. 
8. User-centered evaluation

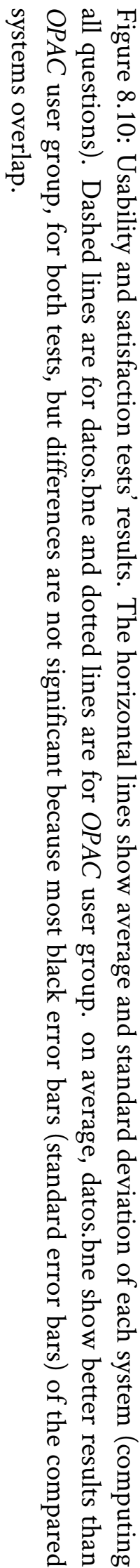

Satisfaction average value
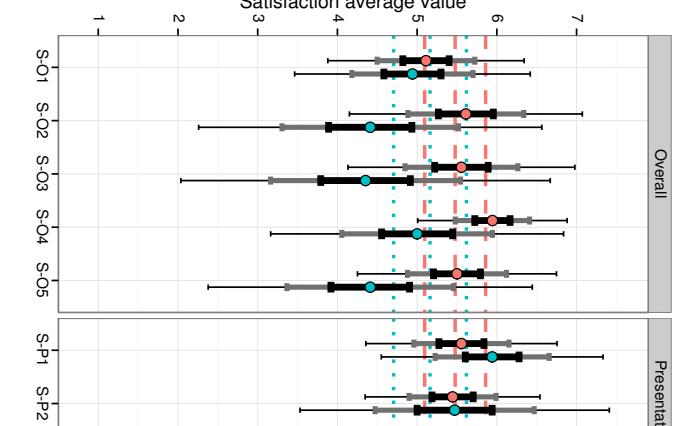

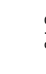

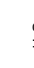

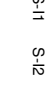

$\frac{\varphi}{\omega}$

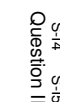

$\bar{\sigma}^{\circ}$

足

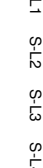

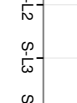

0
5
5
$c$

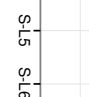

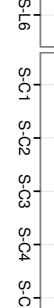

\&

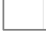

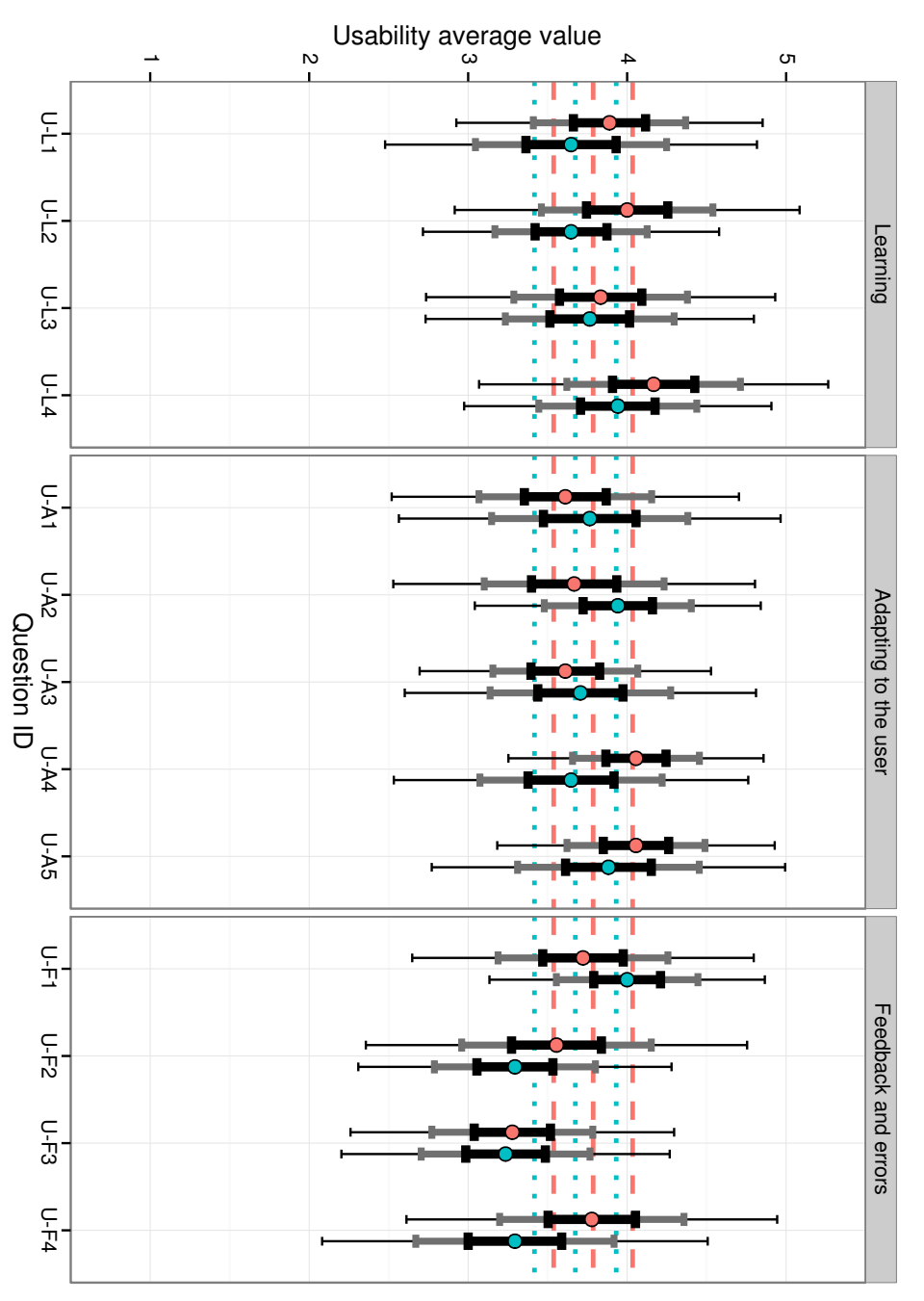

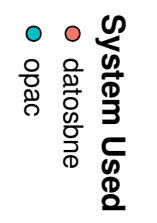




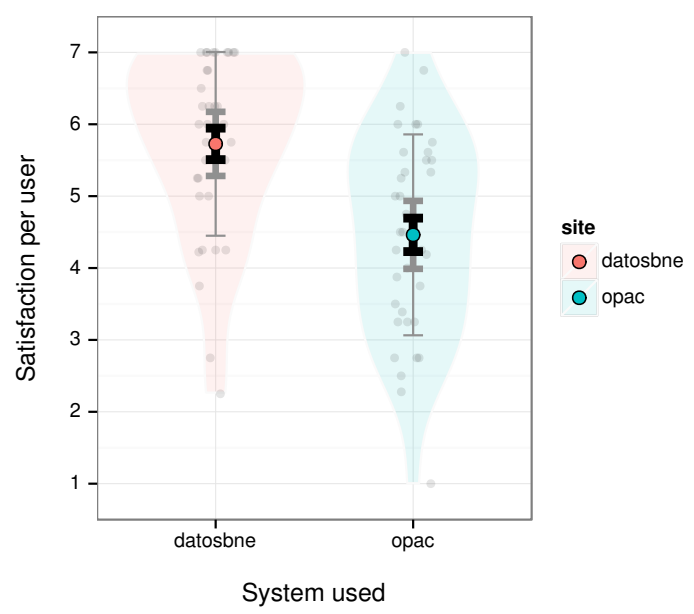

Figure 8.11: Average satisfaction per task for OPAC and datosbne users . Notice that the average values of this coarse-grain satisfaction measure are aligned with the values obtained in the overall facet of the fine-grain satisfaction shown in Figure 8.3.1. Results are also consistent with Figure 8.9.

\subsection{Summary}

Few semantic-based bibliographic systems and applications are accompanied by formal user studies. This situation leaves the question about their potential enhancement of user experiences unanswered. To mitigate this lack of empirical data we present in this chapter two user-centered within-group empirical studies. To our knowledge, our study is the first to compare a novel semantic-based bibliographic system with a traditional, widely deployed online access catalogue. We defined three core hypotheses, or research goals, and we designed a controlled experiment with 72 participants to evaluate our hypotheses following standard and well-established methodologies. These core hypotheses shared the underlying assumption that semantic and linked data technologies provide a good basis for building novel applications to satisfy the users' information needs.

The results of our first empirical experiment, a task-solving scenario linked to the digital humanities and information sciences, provide both quantitative and qualitative evidences for our initial hypotheses. In particular, regarding task completion time, TCT (hypothesis 5a) and visited pages, TPV (hypothesis 5b), our results show a statistically significant evidence that semantic and linked data technologies provide a positive impact for a typical search and retrieval scenario. The analysis of users' characteristics show that age is a factor that influences the time required to complete the 
proposed scenario. Other characteristics such as course, or previous experience with the OPAC or datos.bne.es, do not have a significant effect. Nevertheless, our results suggest that datos.bne.es provides better results compared to OPAC, not only for specialists (e.g., librarians) but for a wider range of users. Additionally, we provided an analysis in a per-task evaluation and related it to the underlying ontology and the core UI features of the datos.bne.es service. Notably, our results indicate that the more complex the navigation the higher the difference between the systems is. Our first experiment also gathered insightful information from a set of qualitative responses provided by the participants. Our results show that task user satisfaction, TUS (hypothesis $5 \mathrm{c}$ ), after completing each task, is significantly higher with the datos.bne.es service.

Furthermore, we conducted a second study, a fine-grained usability and user satisfaction test. The results of our study are complementary to the in-scenario task user satisfaction evaluation, TUS (hypothesis $5 \mathrm{c}$ ) but add up a more detailed assessment. In particular, while the in-scenario results indicate a statistically significant increase in user satisfaction the fine-grained satisfaction questionnaire suggests also an increase in the average satisfaction. However, the mean values obtained in the category overall for the coarse-grain user satisfaction are in line with the mean values obtained for the fine-grained satisfaction study. Regarding the fine-grained usability tests, our results suggest also a better average score. 


\section{Conclusion}

This thesis addresses different challenges in the area ontology-based library data generation, access and exploitation. These challenges converge to the three main research problems we discussed in Chapter 3: (P1) Systematically analyzing and modelling library catalogue data using ontologies to enrich their semantics; (P2) Formally defining the mapping and transformation rules; and (P3) Understanding the impact of ontology-based library data access in end-user applications.

As shown throughout this document, our thesis formulates and evaluates five hypotheses, and presents several contributions to address these research problems. We review these hypotheses and contributions below, in the light of the evidence shown throughout the previous chapters and the evaluations performed.

\subsection{Hypotheses and contributions}

The main contribution of this thesis is the marimba-framework, a framework for ontology-based library data generation, access and exploitation. This framework encompasses several contributions that are aimed at addressing the three aforementioned research problems. Following the design-science research methodology described in Section 3.4, we have evaluated our five hypotheses using the marimba-framework for the large scale linked data project datos.bne.es.

\subsubsection{Systematically analyzing and modelling library catalogue data using ontologies}

As discussed in Chapter 2, previous works dealing with the transformation of library catalogues using semantics lack a methodological approach to deal with inherent com- 
plexity and heterogeneity of catalogue data sources and library ontologies. In this thesis, we have approached this limitation from two different but related angles: $i$ ) from the perspective of ontology development methods that enable the domain experts to deal with data heterogeneity and complexity; and $i i$ ) from the perspective of methods and measures that allow for more sophisticated analysis and comparison of library ontologies. Along these two lines, we have formulated two hypotheses and proposed a series of contributions that we discuss below.

\section{H1. Systematic approaches based on catalogue data and domain expert inputs can produce} an ontology with reasonable quality. This hypothesis is motivated by the limitations of existing works identified and discussed in Section 2.2.1 that can be summarized as follows:

Limitation 1. First, several reviewed works highlight that library data sources, although relatively structured and standardized, show a high degree of data and structural heterogeneity (Manguinhas et al. [2010], Coyle [2011], Takhirov et al. [2012]). However, none of the existing works tackles this heterogeneity from a methodological perspective and they deal with this heterogeneity either from a theoretical perspective (Coyle [2011]), or with hand-crafted solutions for specific use cases (Manguinhas et al. [2010], Takhirov et al. [2012]).

Main contributions. To overcome this limitation, in Chapter 4 we have proposed a contribution, which we called marimba-mapping. Our contribution includes a schema extraction method to extract the metadata schema from MARC 21 library catalogues, and a mapping template generation method to present the extracted schema in a meaningful way to library experts.

Limitation 2. Second, most of the authors agree that library experts can improve the quality of the process of modelling and transforming library catalogues using semantic models (Hickey et al. [2002], Hegna and Murtomaa [2003], Takhirov et al. [2012]). Unfortunately, none of the previous approaches provides the means to enable the active participation of library experts in this process. Moreover, we have reviewed in Section 2.4.5 existing ontology development methods in the light of the library scenario and we have identified two main gaps. First, none of the approaches provides well-defined mechanisms for extracting and representing evolving requirements, which is a critical aspect for the library domain, as discussed in the first limitation (Limitation 1). Second, there is a lack of guidelines and mechanisms for enabling 
an active participation of domain experts in the ontology development process.

Main contributions. To overcome this limitation, in Chapter 5 we have proposed a novel contribution, which we called marimba-modelling. Our contribution instantiates and extends the well-established NeOn methodology (Suárez-Figueroa et al. [2015]) by providing an ontology-development life-cycle with three major novelties: i) the elicitation and evolution of requirements is supported by the mapping templates generated by the marimba-mapping method; $i$ ) the active participation of library experts in the ontology design process by using the mapping templates; and, iii) an ontology publication activity, to publish the ontology on the Web following linked data best practices. Furthermore, in this thesis we have applied our proposed ontology development life-cycle to design and develop a large library ontology, the BNE ontology. ${ }^{1}$ Finally, the BNE ontology is published on the Web as linked data with an open license, following our proposed ontology publication activity.

Evaluation and discussion. We have evaluated our first hypothesis with the application of our contributions to design and develop the BNE ontology through several iterations as described in Chapter 5. Specifically, in Chapter 5, we have shown the application of the marimba-mapping and the ontology development life-cycle in two iterations. During the first iteration, a team of library experts and ontological engineers designed and developed a FRBR-based ontology. During the second iteration, the team improved, extended, and integrated the previous ontology to implement the BNE ontology, a large library ontology merging concepts from more than ten ontologies. To validate our first hypothesis, in Section 5.6.2, we have performed and discussed the results of a topology-based evaluation of the BNE ontology, with respect to a catalogue of 33 ontology design and development pitfalls. We have performed the evaluation in two iterations following a diagnose and repair process. The results of the evaluation show that $i$ ) no critical issues were found in the BNE ontology, indicating the sufficient quality of the ontology; $i i$ ) seven pitfalls were identified during the first iteration, three of them labelled as important; and, iii) during the second iteration, the important pitfalls were easily repaired, leaving only two minor pitfalls that did not critically affect the quality of the BNE ontology. Thus, the empirical and evaluation results validate our hypothesis and show that a systematic approach based on catalogue data and the inputs of library experts produces an ontology with no critical issues or defects.

\footnotetext{
${ }^{1}$ http://datos.bne.es/def/
} 
H2. Probabilistic topic modelling techniques can produce coherent descriptions of ontologies and perform better in terms of precision than classical term-count based methods for ontology clustering. This hypothesis is motivated by the limitations in the state of the art identified and discussed in Sections 2.1.4 and 2.2.1 that can be summarized as follows.

On the one hand, over the last years a great number of ontologies for modelling library data have been proposed. These library ontologies offer a wide range of frequently overlapping classes and properties to model library data. While this variety is in principle a positive aspect, it also adds complexity to the task of finding, comparing and selecting classes and properties that can potentially be used to model the same entities but use different terminology. On the other hand, existing methods, techniques and tools for ontology reuse, search and similarity are based on either topological features (e.g., links between ontologies), or on term-based similarity measures. However, this type of techniques are limited for the library domain, where many ontologies describe similar entities and concepts using heterogeneous terminology.

Main contributions. To address this limitation, in Chapter 6 we have proposed a novel contribution, which we called marimba-topicmodelling. Our contribution encompasses a method for the extraction of ontology documents from ontology corpora, such as the LOV corpus, their annotation with senses using word-sense disambiguation, and a topic modelling method for short-text documents to train a probabilistic topic model. Furthermore, based on the topic model inference capabilities, we have proposed a novel ontology-based similarity measure based on the Jensen-Shannon divergence. Additionally, in Section 6.5.3 we have proposed two gold-standard datasets for evaluating ontology similarity measures through an ontology clustering task.

Evaluation and discussion. With the above-described contribution, we hypothesize that topic modelling techniques combined with word-sense disambiguation can provide more precise measurements of the similarity between ontologies in hetereogeneous domains like the library domain. In Chapter 6 we have evaluated our main hypothesis by first addressing two sub-hypotheses: (H2a) the annotation of ontology documents with senses can improve the coherence of the extracted topics, and (H2b) senseannotated ontology documents will suffer from data sparsity issues, and, thus, topic models for short-text documents will produce more coherent topics than classical topic models. To validate our two sub-hypotheses, in Section 6.5.2, we have presented an experiment to compare the coherence of different settings of the marimba-topicmodelling method using a state-of-the-art coherence measure (Mimno et al. [2011]). The results showed 
that the combination of a topic model for short-text documents (BTM) with senseannotated documents consistently outperforms all the other settings, namely BTM without non-annotated documents, LDA with sense-annotated documents, and LDA with non-annotated documents. These results provide statistically significant evidence for our two sub-hypotheses on a representative training corpus (i.e., the LOV ontology corpus). Furthermore, even for LDA the experiment showed the increase in coherence using sense-annotated documents, which provides stronger evidence of the positive effect of word-sense disambiguation. Finally, in order to validate our main hypothesis, we have presented a task-based experiment to measure the precision of our proposed topic-based ontology similarity measures. Specifically, we compared our method with the widely-used tf-idf method using different configurations of similarity measures and distance metrics. The results of the experiment with two gold-standards showed that our topic-based ontology similarity measures consistently achieved higher precision for the task of clustering similar ontologies. Furthermore, the results showed that the improved precision of our method is statistically significant in both gold-standard datasets, and the improvement ratio is consistent accross different similarity measures and distance measures.

\subsubsection{Formally defining the mapping and transformation rules}

As discussed in Section 2.2.1, existing methods for transforming library catalogues into richer models rely on ad-hoc, programmatically defined and thus not easily interoperable transformation and mapping rules. On the other hand, as reviewed in Section 2.3.1, in the area of ontology-based data access a great deal of research has focused on mapping relational databases into RDF (W3C R2RML language). Moreover, more recent works have started to explore the mapping of other types of data such as nonrelational databases or hierarchical data formats (Dimou et al. [2014], Michel et al. [2015], Slepicka et al. [2015]). Based on these existing lines of research, in this thesis we have studied the problem of mapping MARC 21 data sources into RDF, formulated two hypotheses and presented a series of contributions that we discuss below.

\section{H3. MARC 21 data sources can be represented with an abstract relational model so that they} can be transformed with an extension of existing mapping languages. As highlighted in Section 2.2.1, there is lack of data model and iteration mechanisms of MARC 21 data sources, which are essential to provide a formal framework for defining a mapping language for MARC 21 data sources into RDF. Moreover, motivated by preliminary 
ideas of Slepicka et al. [2015], we hypothesize that the Nested Relational Model can be used to provide a formal data representation for MARC 21 data sources and in turn be able to design a language for mapping MARC 21 data sources into RDF. However, the work of Slepicka et al. [2015] does not cover a full representation of data in the Nested Relational Model and does not provide the ability of using joins accross data sources.

Main contribution. To tackle these limitations, in Chapter 4, we have proposed a data model based on the Nested Relational Model, which we called marimba-datamodel. Our contribution includes the formal definition of the nested relational model and its application to fully model MARC 21 data sources.

Evaluation and discussion. With the above-described contribution, we hypothesize that MARC 21 data source can be modelled using a formal data model. Specifically, in Section 4.2, we have analyzed and defined the translation of the metadata elements of MARC 21 records into the Nested Relational Model proposed by Makinouchi [1977]. Moreover, we have analytically shown how the Nested Relational Model can be formally used to cover the complete record specification of the MARC 21 standard. This formal modelling provides a framework for defining the core components of a mapping language that is both formally grounded by a recursive algebra presented in 4.4.1, and it is aligned to existing standard mapping languages such as the W3C R2RML recommendation.

\section{H4. A minimal query and a mapping language can be designed to define mapping rules be-} tween MARC 21 data sources and RDF. Once we have provided a formal relation data model for MARC 21 data sources, we hypothesize that we can extend the W3CR2RML mapping language with minimal changes to deal with MARC 21 data sources. However, to provide such mapping language extension we need to tackle two existing limitations. First, the closest approach to this problem (Slepicka et al. [2015]) does not provide a formal language to iterate and query data in the Nested Relational Model. Second, the W3C R2RML language and the W3C R2RML processor need to be analyzed and modified in the light of the properties of the Nested Relational Model.

Main contributions. To tackle these limitations, we have approached the problem of querying and mapping MARC 21 data sources in three steps. First, in Section 4.4, we have proposed a minimal query language, which we called marimba-sql. The marimba-sql query language is defined as a subset of the SQL/NF query language proposed by Colby [1989]. Second, in Section 4.5 we have proposed marimba-rml, 
a mapping language that extends the W3C R2RML language in order to deal with data sources in the Nested Relational Model. Finally, we have proposed a marimbarml mapping processor to transform MARC 21 data sources into RDF using mapping documents defined in the marimba-rml.

Evaluation and discussion. With the above-described contributions, in Chapter 4, we have shown that a query language and mapping language can be defined to map and transform MARC 21 data sources with minimal changes to the W3C R2RML recommendation. First, in Section 4.4.3, we have defined the syntax marimba-sql query language and provided the complete BNF grammar definition in Apendix X. Second, we have shown in Section 4.4.4 how the expressions in the marimba-sql language can be translated into the recursive algebra defined in Section 4.4.1. Third, we have defined the syntax of the marimba-rml mapping language in Section 4.5.1, which implies minimal changes to the W3C R2RML language syntax. Finally, in Section 4.5.2 we have defined an marimba-rml processor, which defines the operational semantics of the marimba-rml language.

\subsubsection{Understanding the impact of ontology-based library data access in end-user applications}

The application of ontologies and linked data come with the promise of providing enhanced access, user experiences and reuse of library assets on the Web, as reflected in the final report of the W3C LLD Incubator Group (W3C [2011]) and Alemu et al. [2012]. Despite this intuition, as highlighted in Section 2.2.1, there is a lack of indepth studies to evaluate the effects of these technologies on end-users. To tackle this limitation, in this thesis we have formulated and evaluated the following hypothesis.

H5. The application of semantic technologies to end-user library application results in significantly better user satisfaction and ef ficiency in completing information retrieval tasks, with respect to classical end-user library applications. In this thesis, we hypothesize that the application of semantic technologies in general, and the marimba-framework in particular, to library end-user systems can bring noticeable benefits to users in terms of overall experience. In order to gather insights to ground this intution, in Chapter 8 , we have presented two large experiments with real users to perform a comparitive analysis of two large systems of the National Library of Spain: the traditional online catalogue (OPAC) and the datos.bne.es service described in Chapter 7. 
Main contributions. In Chapter 8, we have designed and performed two experiments to evaluated different standard measures related to user efficiency, user experience and usability. The first experiment was intended to measure the satisfaction of user needs in a task-based realistic scenario. The second experiment was intended to provide a more detailed evaluation of usability and user satisfaction measures. With these two experiments, our study is the first to compare a novel semantic-based bibliographic system with a traditional, widely deployed online access catalogue.

Evaluation and discussion. The results of our first empirical experiment, a tasksolving scenario linked to the digital humanities and information sciences, provide both quantitative and qualitative evidences for our hypothesis (H5). Regarding quantitative measures related to efficiency, we have measured the task completion time, TCT (hypothesis 5a) and visited pages, TPV (hypothesis $5 \mathrm{~b}$ ). Our results show a statistically significant evidence that semantic and linked data technologies provide a positive impact in efficiency for a typical search and retrieval scenario. Our first experiment also gathered insightful information from a set of qualitative responses provided by the participants. Our results show that task user satisfaction, TUS (hypothesis $5 \mathrm{c}$ ), after completing each task, is significantly higher with the datos.bne.es service.

Regarding the second experiment, a fine-grained usability and user satisfaction test, the results of this experiment add up a more detailed assessment to the results of the task user satisfaction evaluation performed during the first experiment (TUS (hypothesis $5 \mathrm{c})$ ). In particular, while the in-scenario results indicate a statistically significant increase in user satisfaction the fine-grained satisfaction questionnaire suggests also an increase in the average satisfaction. Finally, regarding the fine-grained usability tests, our results suggest also a better average score.

\subsection{Impact}

Besides from the above-described contributions, the techniques and technologies presented in this thesis and their deployment on the National Library of Spain information systems have produced a positive impact on the way the National Library interacts with end-users, other institutions, and the society at large.

First, since the deployment of the datos.bne.es service presented in Chapter 7, the number of daily users have increased steadily. As of July 2016 more the service has received more than 1 million visitors with an average of 45,000 visitors per month. More importantly, the number of visitors accessing the online catalogue directly through 
search engines (i.e., Google, Bing, Yahoo, etc.) has doubled, which highlights the increased visibility brought by the techniques presented in this thesis. Figure 9.1 shows a comparison between the daily users of the datos.bne.es and catalogo.bne.es online services coming directly from search engines from November 2015 to June 2016. The figure shows the exact daily users for each service (i.e., the dots in blue correspond to datos.bne.es and the dots in red correspond to catalogo.bne.es). Moreover, the lines in the graph, obtained by a linear model, show that the access trends for both online services are very similar and datos.bne.es is very close to catalogo.bne.es in terms of daily users. Specifically, since its release the datos.bne.es services has received more than 850,000 users from search engines while catalogo.bne.es has received around 950,000 visitors. More interestingly, while almost $99 \%$ of the visits to catalogo.bne.es correspond to queries with general keywords like "catalogo bne" or "libros bne", more than $20 \%$ of the visits to datos.bne.es are unique visits corresponding to very precise keywords such as "author of the song Bolero de la Calamocha". This clearly indicates that the techniques presented in this thesis are increasing the visibility of the so-called "long-tail" resources. ${ }^{2}$

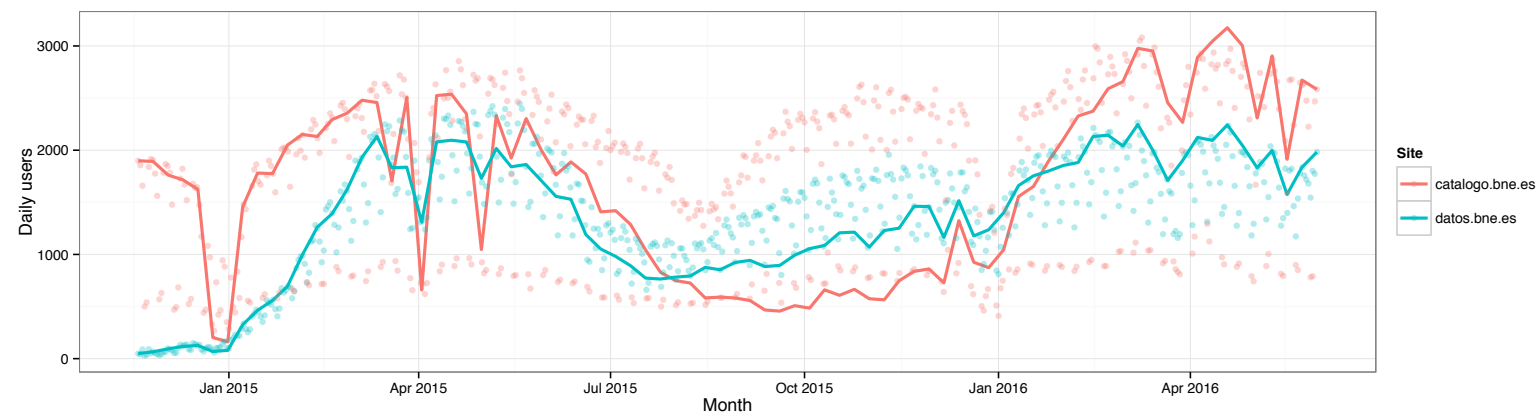

Figure 9.1: Statistics of daily users of the datos.bne.es and catalogo.bne.es portals coming directly from search engines.

Second, the publication of the BNE catalogue as linked data is increasing the reuse of the BNE resources by other cultural institutions in Spain. Two notable examples of this trend are: $i$ ) the digital library of the region of "Galicia" , which reuses the BNE authorities to complete the information about works, persons and organizations; and ii) "Pares", the digital portal for the Spanish National Archives, which reuses

\footnotetext{
${ }^{2}$ Most of the queries in the $20 \%$ of visitors correspond to either $i$ ) very specific queries with patterns like "author of...", or ii) rare resources such as authors, or music bands with very few catalogued works.

${ }^{3}$ http: //www.galiciana.bibliotecadegalicia.xunta.es (Last viewed 1st July 2016).
} 
datos.bne.es data for automatically completing bibliographic references. Since the release of datos.bne.es, the BNE has recognized the value of the technologies presented in this thesis as a mechanism to better interact with other cultural actors in Spain.

Finally, thanks to the technologies implemented and deployed in this thesis, the National Library has reached a wider audience and increased the value of their resources outside the traditional channels (e.g., the research community). A notable example of this trend is the story published by the Spanish Broadcast Agency for Radio and Television ${ }^{4}$, describing datos.bne.es as a novel, more intuitive online catalogue. Finally, another example is the usage of datos.bne.es service in the context of a citizen science initiative with kids, called "Street detective", which received the Fujitsu Laboratories of Europe Innovation Award in 2015.

\subsection{Future directions}

The work presented in this thesis provides contributions that enable mapping and transforming library catalogues into RDF using ontologies in a principled and systematic way. Moreover, our work contributes to the application of semantic technologies to end-user online library services and provides several evidences of their positive impact through the extensive evaluation presented in Chapter 8. Nevertheless, we have already highlighted throughout the thesis several limitations, open issues and new research directions. In this section, we discuss and outline a set of future directions with regard to the contributions of this thesis. We organize the discussion into the following lines of research: i) query and mapping languages, ii) Machine learning and probabilistic approaches, iii) Data linking and enrichment, iv) End-user library applications and their evaluation.

\subsubsection{Query and mapping languages}

In Chapter 4 we have proposed the marimba-sql and marimba-sql languages to query and map existing library data sources into RDF. These two languages are backed by a formal relational model, the Nested Relational Model, and a recursive nested relational algebra. In this thesis, we have demonstrated, both analytically and practically, their suitability for transforming library records into RDF. Moreover, we have provided and applied an implementation of these ideas through the marimba-framework and

\footnotetext{
${ }^{4}$ The story, in Spanish, is available at http://www.rtve.es/noticias/20150310/ biblioteca-nacional-estrena-catalogo-online-mas-intuitivo (Last viewed 1st July 2016).
} 
its marimba-rml processor. However, there are several open issues, challenges and interesting future lines of research that we ellaborate below.

First, we have demonstrated the applicability of marimba-sql and marimba-rml to the transformation of library records. However, these two languages can be applied, in principle, to any type of nested data format such as JSON or XML. In fact, these ideas have been already explored by one of the works reviewed in Chapter 2, namely KR2RML. As we have highlighted in Chapter 4, marimba-sql and marimba$\mathrm{rml}$ provide a step forward towards a more general solution to deal with nested data, which overcomes some of the limitations of KR2RML, namely its ability to deal with joins and the lack of sophisticated query mechanisms. Likewise, we have applied the marimba-framework within the library domain, but there are many other domains where the same techniques could be applied such as archives, museums, or mass media.

Second, in this thesis we have not investigated any of the possible directions of query and transformation optimizations that the application of the NRM and the recursive algebra could bring to the RDF generation process. The research on optimization techniques to both the query and transformation processes is a highly relevant direction of research. As a starting point, the research on this direction can build on the research presented in Melnik et al. [2010]. Melnik et al. [2010] proposed a novel approach based on the nested relational model to enable a highly scalable and optimized mechanism for querying nested data in very large datasets. We believe that this kind of approach brings new possibilities to enable large-scale and real-time transformation of nested relational data into RDF.

\subsubsection{Machine learning and probabilistic approaches}

In Chapter 6, we have introduced a novel approach to model ontologies using probabilistic techniques (i.e., probabilistic topic models). Our approach combines techniques from two different areas of artificial intelligence: classical knowledge representation and statistical approaches. Our work opens up new directions in exploring the frontiers between these two research areas. We ellaborate on some of these directions below.

First, the application of topic models and word-sense disambiguation to represent the knowledge embedded in formal ontologies has several potential applications. For instance, the techniques presented in this thesis can provide new mechanisms for discoverying ontologies by providing web services that compare and propose ontologies 
based on their thematic similarities. Moreover, this kind of service can help existing ontology alignment tools to find potential candidate ontologies during the alignment process. Also, ontology repositories such as LOV can benefit from our techniques to provide enhanced ways of finding and presenting the results to final users thus facilitating the ontology reuse task.

Second, learning approaches can be used to facilitate the mapping process described in Chapter 4. One of the main contributions of this thesis is the participation of domain experts in the mapping and transformation process. To this end, domain experts provide highly accurate inputs to the mapping process, and such inputs can in turn be used to train machine learning algorithms such as conditional random fields. These learning algorithms can then be used to automatize different aspects of the mapping process, thus reducing the manual effort and potentially increasing the quality of the transformed data. A clear candidate for the application of machine learning algorithms is the classification process, in which the domain experts establish the mappings for classifying MARC 21 records into ontology classes.

Finally, another interesting direction is the embedding of the data graphs generated by the marimba-framework. Knowledge graph embedding is a technique to represent RDF or knwoledge graphs in vector spaces. This technique has proven to be very powerful for tasks such as knowledge graph completion, knowledge fusion, entity linking and link prediction. The combination of these embeddings and the rich semantics provided by ontologies open up new possibilities for providing more sophisticated search services, and increasing the quality of the data.

\subsubsection{Data linking and enrichment}

In Chapter 7, we have introduced the data linking and enrichment task. This task supports a central principle of the linked data paradigm, namely providing links to external sources on the Web that provide additional information about a resource. In this thesis, we have shown how to reuse authoritative data sources such as VIAF and DBpedia to boost the data linking and enrichment processes. Nevertheless, exclusively reusing authoritative data sources such as the VIAF mappings hinders the potential of the produced linked data.

Therefore, we have started investigating on automatic linking algorithms during the second research stay at the EXMO group of INRIA Grenoble. Specifically, we have investigated the following lines of research: $i$ ) the automatic selection of properties to be used for data linking using the concept of "keys", that uniquely identify RDF 
resources; $i i)$ the discovery of links accross datasets in different languages, or crosslingual linking; and $\mathrm{iii}$ ) the generation of gold standards for evaluating (cross-lingual) linking algorithms using library datasets such as the data.bnf.fr, datos.bne.es and VIAF. We plan to work on this interesting area and continue our collaboration with the EXMO group.

\subsubsection{End-user library applications and their evaluation}

The studies presented in Chapter 8 evidence the benefits of the application of ontologybased and linked data approaches to library applications. In this thesis, we have shown one application and the integration of ontology-based techniques in classical information search and retrieval techniques (e.g., the ranking algorithm based on the ontology relationships shown in Chapter 7). We believe that the techniques proposed in this thesis, to increase the semantics and connectivity of library resources using ontologies, bring up new possibilities of developing more intuitive and intelligent applications. As a first natural step, we plan to provide new functionalities for semantic search within datos.bne.es by further integrating the ontological knowledge and further exploiting the graph nature of the data. A logical step would be identifying ontological concepts and properties in user queries and using that knowledge to provide more precise answers to queries such as "works of Miguel de Cervantes" or "topics related to Don Quixote".

Regarding the evaluation, our studies leave some open questions for the future. In particular, we could perform wider usability and satisfaction tests in order to gather other significant evidences and insights. Also, a further in-depth analysis of the impact of semantic modeling and UI features can help us to better understand the implications of these technologies on end user applications and thus lead to better semantic solutions. Finally, our results suggest that the new system has a positive impact on users with different backgrounds and skills and a broader study with different user profiles (e.g., including more general public) can bring stronger evidence of such impact. 

Appendix $\mathbf{A}$ 


\section{marimba-sql BNF}

We introduce the grammar of marimba-sql in BNF notation. This grammar is a subset of the grammar presented by Roth et al. [1987].

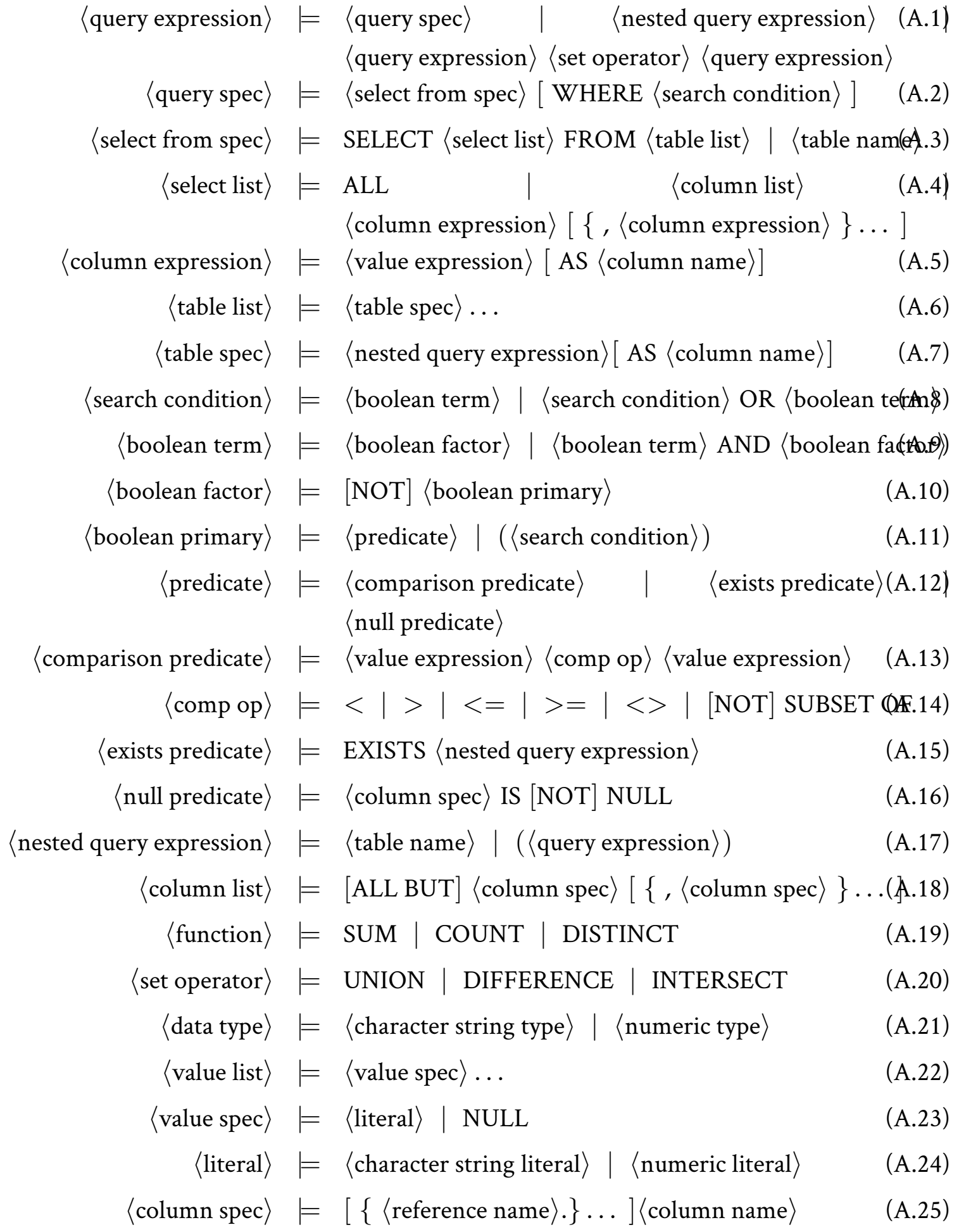




\section{Bibliography}

Trond Aalberg. A process and tool for the conversion of marc records to a normalized frbr implementation. In Shigeo Sugimoto, Jane Hunter, Andreas Rauber, and Atsuyuki Morishima, editors, Digital Libraries: Achievements, Challenges and Opportunities: 9th International Conference on Asian Digital Libraries, ICADL 2006, Kyoto, Japan, November 27-30, 2006. Proceedings, pages 283-292. Springer Berlin Heidelberg, Berlin, Heidelberg, 2006.

Trond Aalberg, Frank Berg Haugen, and Ole Husby. A Tool for Converting from MARC to FRBR. In Research and Advanced Technology for Digital Libraries, pages 453-456. Springer, 2006.

Marco D Adelfio and Hanan Samet. Schema extraction for tabular data on the web. Proceedings of the VLDB Endowment, 6(6):421-432, 2013.

Harith Alani, Christopher Brewster, and Nigel Shadbolt. Ranking ontologies with aktiverank. In The Semantic Web-ISWC 2006, pages 1-15. Springer, 2006.

Getaneh Alemu, Brett Stevens, Penny Ross, and Jane Chandler. Linked data for libraries: Benefits of a conceptual shift from library-specific record structures to rdfbased data models. New Library World, 113(11/12):549-570, 2012.

Carlo Allocca, Mathieu d'Aquin, and Enrico Motta. Towards a formalization of ontology relations in the context of ontology repositories. In Ana Fred, JanL.G. Dietz, Kecheng Liu, and Joaquim Filipe, editors, Knowledge Discovery, Knowlege Engineering and Knowledge Management, volume 128 of Communications in Computer and Information Science, pages 164-176. Springer Berlin Heidelberg, 2011. ISBN 978-3642-19031-5. 
Carlo Allocca, Mathieu d'Aquin, and Enrico Motta. Impact of using relationships between ontologies to enhance the ontology search results. In Elena Simperl, Philipp Cimiano, Axel Polleres, Oscar Corcho, and Valentina Presutti, editors, The Semantic Web: Research and Applications, volume 7295 of Lecture Notes in Computer Science, pages 453-468. Springer Berlin Heidelberg, 2012. ISBN 978-3-642-30283-1.

Martin Andersson. Extracting an entity relationship schema from a relational database through reverse engineering. International Journal of Cooperative Information Systems, 04(02n03):259-285, 1995.

Marcelo Arenas, Alexandre Bertails, Eric Prud'hommeaux, and JF Sequeda. A Direct Mapping of Relational Data to RDF, W3C Recommendation 27 September 2012. World Wide Web Consortium. http://www.w3.org/TR/2012/REC-rdb-directmapping-20120927, 2013.

Arthur Asuncion, Max Welling, Padhraic Smyth, and Yee Whye Teh. On smoothing and inference for topic models. In Proceedings of the Twenty-Fifth Conference on Uncertainty in Artificial Intelligence, pages 27-34. AUAI Press, 2009.

Ghislain Auguste Atemezing, Oscar Corcho, Daniel Garijo, José Mora, María PovedaVillalón, Pablo Rozas, Daniel Vila-Suero, and Boris Villazón-Terrazas. Transforming meteorological data into linked data. Semantic Web, 4(3):285-290, 2013.

Ghislain Auguste Atemezing, Bernard Vatant, Raphaël Troncy, and Pierre-Yves Vanderbussche. Harmonizing services for LOD vocabularies: a case study. In WASABI 2013, Workshop on Semantic Web Enterprise Adoption and Best Practice, Colocated with ISWC 2013 Workshop, October 22, 2013, Sydney, Australia, 102013.

Sören Auer. The RapidOWL Methodology-Towards Agile Knowledge Engineering. In 15th IEEE International Workshops on Enabling Technologies: Infrastructures for Collaborative Enterprises (WETICE 2006), 26-28 June 2006, Manchester, United Kingdom, pages 352-357, 2006.

Franz Baader, Diego Calvanese, Deborah L. McGuinness, Daniele Nardi, and Peter F. Patel-Schneider, editors. The Description Logic Handbook: Theory, Implementation, and Applications, 2003. Cambridge University Press. ISBN 0-521-78176-0.

Ricardo Baeza-Yates, Berthier Ribeiro-Neto, et al. Modern information retrieval, volume 463. ACM press New York, 1999. 
Jesús Barrasa, Óscar Corcho, and Asunción Gómez-pérez. R2o, an extensible and semantically based database-to-ontology mapping language. In in In Proceedings of the 2nd Workshop on Semantic Web and Databases(SWDB2004, pages 1069-1070. Springer, 2004.

C. Batini, M. Lenzerini, and S. B. Navathe. A comparative analysis of methodologies for database schema integration. ACM Comput. Surv., 18(4):323-364, December 1986a. ISSN 0360-0300.

Carlo Batini, Maurizio Lenzerini, and Shamkant B. Navathe. A comparative analysis of methodologies for database schema integration. ACM computing surveys (CSUR), 18(4):323-364, 1986b.

Rick Bennett, Brian F Lavoie, and Edward T OI neill. The concept of a work in worldcat: an application of frbr. Library Collections, Acquisitions, and Technical Services, 27(1):45-59, 2003.

Tim Berners-Lee. Linked data, 2006. URL http://www.w3.org/DesignIssues/ LinkedData.htm. http://www.w3.org/DesignIssues/LinkedData.html accessed 2011-08-12.

Tim Berners-Lee, James Hendler, Ora Lassila, et al. The semantic web. Scientific american, 284(5):28-37, 2001.

Diego Berrueta and Jon Phipps. Best practice recipes for publishing rdf vocabularies. World Wide Web Consortium, Note NOTE-swbp-vocab-pub-20080828, August 2008. URL http: //www.w3 . org/TR/2008/NOTE-swbp-vocab-pub-200808.

Christian Bizer and Andy Seaborne. D2rq-treating non-rdf databases as virtual rdf graphs. In Proceedings of the 3rd international semantic web conference (ISWC2004), volume 2004. Citeseer Hiroshima, 2004.

David M Blei. Probabilistic topic models. Communications of the ACM, 55(4):77-84, 2012.

David M Blei, Andrew Y Ng, and Michael I Jordan. Latent dirichlet allocation. the Journal of machine Learning research, 3:993-1022, 2003.

Ilaria Bordino, Carlos Castillo, Debora Donato, and Aristides Gionis. Query similarity by projecting the query-flow graph. In Proceedings of the 33rd international ACM 
SIGIR conference on Research and development in information retrieval, pages 515-522. ACM, 2010.

Christopher Brewster, Harith Alani, Srinandan Dasmahapatra, and Yorick Wilks. Data driven ontology evaluation. 2004.

John Brooke. Sus-a quick and dirty usability scale. Usability evaluation in industry, 189 (194):4-7, 1996.

John Brooke. Sus: a retrospective. Journal of Usability Studies, 8(2):29-40, 2013.

Wray L. Buntine. Operations for learning with graphical models. JAIR, 2:159-225, 1994.

Andrew Burton-Jones, Veda C Storey, Vijayan Sugumaran, and Punit Ahluwalia. A semiotic metrics suite for assessing the quality of ontologies. Data $\&$ Knowledge Engineering, 55(1):84-102, 2005.

Eric J Byrne. A conceptual foundation for software re-engineering. In Software Maintenance, 1992. Proceerdings., Conference on, pages 226-235. IEEE, 1992.

Gong Cheng and Yuzhong Qu. Relatedness between vocabularies on the web of data: A taxonomy and an empirical study. Web Semantics: Science, Services and Agents on the World Wide Web, 20:1-17, 2013.

Gong Cheng, Weiyi Ge, and Yuzhong Qu. Falcons: searching and browsing entities on the semantic web. In Proceedings of the 17th international conference on World Wide Web, pages 1101-1102. ACM, 2008.

Jing Cheng, Xiao Hu, and P Bryan Heidorn. New measures for the evaluation of interactive information retrieval systems: Normalized task completion time and normalized user effectiveness. Proceedings of the American Society for Information Science and Technology, 47(1):1-9, 2010.

Xueqi Cheng, Xiaohui Yan, Yanyan Lan, and Jiafeng Guo. Btm: Topic modeling over short texts. Knowledge and Data Engineering, IEEE Transactions on, 26(12):2928-2941, 2014.

John P. Chin, Virginia A. Diehl, and Kent L. Norman. Development of an instrument measuring user satisfaction of the human-computer interface. In Elliot Soloway, Douglas Frye, and Sylvia B. Sheppard, editors, Interface Evaluations. Proceedings of 
ACM CHI'88 Conference on Human Factors in Computing Systems, pages 213-218, 1988. June 15-19, 1988. Washington, DC, USA.

Latha S. Colby. A recursive algebra and query optimization for nested relations. In Proceedings of the 1989 ACM SIGMOD International Conference on Management of Data, SIGMOD '89, pages 273-283, New York, NY, USA, 1989. ACM. ISBN 0-89791317-5.

Karen Coyle. MARC21 as Data: A Start. Code4lib journal, (14), 2011. URL http: //journal.code4lib.org/articles/5468.

Mathieu d'Aquin and Enrico Motta. Watson, more than a semantic web search engine. Semantic Web Journal, 2(1):55-63, 2011.

Souripriya Das, Seema Sundara, and Richard Cyganiak. R2rml: Rdb to rdf mapping language (w3c recommendation), 2012. URL http: //www.w3 . org/TR/r2rml/.

Chris J Date and Hugh Darwen. A guide to the SQL Standard: a user's guide to the standard relational language SQL, volume 55822. Addison-Wesley Longman, 1993.

Jérôme David and Jérôme Euzenat. Comparison between ontology distances (preliminary results). In Amit Sheth, Steffen Staab, Mike Dean, Massimo Paolucci, Diana Maynard, Timothy Finin, and Krishnaprasad Thirunarayan, editors, The Semantic Web - ISWC 2008, volume 5318 of Lecture Notes in Computer Science, pages 245-260. Springer Berlin Heidelberg, 2008. ISBN 978-3-540-88563-4.

Jérôme David, Jérôme Euzenat, François Scharffe, and Cássia Trojahn Dos Santos. The alignment api 4.0. Semantic web journal, 2(1):3-10, 2011.

Luciano Frontino de Medeiros, Freddy Priyatna, and Oscar Corcho. Mirror: Automatic $\mathrm{r} 2 \mathrm{rml}$ mapping generation from relational databases. In Engineering the Web in the Big Data Era, pages 326-343. Springer, 2015.

Anastasia Dimou, Miel Vander Sande, Pieter Colpaert, Ruben Verborgh, Erik Mannens, and Rik Van de Walle. Rml: a generic language for integrated rdf mappings of heterogeneous data. In Proceedings of the 7th Workshop on Linked Data on the Web (LDOW2014), Seoul, Korea, 2014.

Li Ding, Rong Pan, Tim Finin, Anupam Joshi, Yun Peng, and Pranam Kolari. Finding and ranking knowledge on the semantic web. In Yolanda Gil, Enrico Motta, 
V.Richard Benjamins, and MarkA. Musen, editors, The Semantic Web $\square$ ISWC 2005, volume 3729 of Lecture Notes in Computer Science, pages 156-170. Springer Berlin Heidelberg, 2005. ISBN 978-3-540-29754-3.

Gordon Dunsire and Mirna Willer. Initiatives to make standard library metadata models and structures available to the semantic web. In Proceedings of the IFLA2010, 2010. URL http://bit.1y/bød3iv.

A Duque-Ramos, U López, JT Fernández-Breis, and R Stevens. A square-based quality evaluation framework for ontologies. In OntoQual 2010-Workshop on Ontology Quality at the 17th International Conference on Knowledge Engineering and Knowledge Management (EKAW 2010), 2010.

B. Efron and R. Tibshirani. Bootstrap methods for standard errors, confidence intervals, and other measures of statistical accuracy. Statistical Science 1(1), art. Publised by Institute of Mathematical Statistics, Feb 1986. URL http://www. jstor .org/ stable/2245500.

Dominik Maria Endres and Johannes E Schindelin. A new metric for probability distributions. IEEE Transactions on Information theory, 2003.

Miriam Fernández, Chwhynny Overbeeke, Marta Sabou, and Enrico Motta. What makes a good ontology? a case-study in fine-grained knowledge reuse. In The semantic web, pages 61-75. Springer, 2009.

Mariano Fernández-López, Asunción Gómez-Pérez, and Natalia Juristo. METHONTOLOGY: from ontological art towards ontological engineering. In Proceedings of the Ontological Engineering AAAI97 Spring Symposium Series. American Asociation for Artificial Intelligence, 1997.

Alfio Ferrara, Andriy Nikolov, and François Scharffe. Data linking for the semantic web. Semantic Web: Ontology and Knowledge Base Enabled Tools, Services, and Applications, 169, 2013.

Nuno Freire, José Borbinha, and Pável Calado. Identification of frbr works within bibliographic databases: An experiment with unimarc and duplicate detection techniques. In Asian Digital Libraries. Looking Back 10 Years and Forging New Frontiers, pages 267-276. Springer, 2007. 
Marc Friedman, Alon Y Levy, Todd D Millstein, et al. Navigational plans for data integration. AAAI/IAAI, 1999:67-73, 1999.

Aldo Gangemi. Ontology design patterns for semantic web content. In The Semantic Web-ISWC 2005, pages 262-276. Springer, 2005.

A. Ghazvinian, N.F. Noy, M.A. Musen, et al. How orthogonal are the obo foundry ontologies. J Biomed Semantics, 2(Suppl 2):S2, 2011.

Asunción Gómez-Pérez. Towards a framework to verify knowledge sharing technology. Expert Systems with Applications, 11(4):519-529, 1996.

Asunción Gómez-Pérez, Daniel Vila-Suero, Elena Montiel-Ponsoda, Jorge Gracia, and Guadalupe Aguado de Cea. Guidelines for multilingual linked data. In 3rd International Conference on Web Intelligence, Mining and Semantics, WIMS '13, Madrid, Spain, June 12-14, 2013, page 3, 2013.

Jorge Gracia and Kartik Asooja. Monolingual and cross-lingual ontology matching with CIDER-CL: Evaluation report for OAEI 2013. In Proc. of 8th Ontology Matching Workshop (OM'13), at 12th International Semantic Web Conference (ISWC'13), Syndey (Australia), volume 1111. CEUR-WS, ISSN-1613-0073, October 2013.

Jorge Gracia, Elena Montiel-Ponsoda, Daniel Vila-Suero, and Guadalupe Aguado-deCea. Enabling Language Resources to Expose Translations as Linked Data on the Web. In Proceedings of the Ninth International Conference on Language Resources and Evaluation (LREC-2014), Reykjavik, Iceland, May 26-31, 2014., pages 409-413, 2014a.

Jorge Gracia, Daniel Vila-Suero, John Philip McCrae, Tiziano Flati, Ciro Baron, and Milan Dojchinovski. Language resources and linked data: A practical perspective. In Knowledge Engineering and Knowledge Management - EKAW 2014 Satellite Events, VISUAL, EKM1, and ARCOE-Logic, Linköping, Sweden, November 24-28, 2014. Revised Selected Papers., pages 3-17, 2014b.

Thomas L Griffiths and Mark Steyvers. Finding scientific topics. Proceedings of the National Academy of Sciences, 101(suppl 1):5228-5235, 2004.

Thomas R Gruber. A translation approach to portable ontology specifications. Knowledge acquisition, 5(2):199-220, 1993. 
Michael Grüninger and Mark S. Fox. Methodology for the design and evaluation of ontologies. In IJCAI'95, Workshop on Basic Ontological Issues in Knowledge Sharing, 1995.

Nicola Guarino and Christopher A Welty. An overview of ontoclean. In Handbook on ontologies, pages 201-220. Springer, 2009.

Claudio Gutierrez, Carlos Hurtado, and Alberto O Mendelzon. Foundations of semantic web databases. In Proceedings of the twenty-third ACM SIGMOD-SIGACT-SIGART symposium on Principles of database systems, pages 95-106. ACM, 2004.

Alon Y. Halevy. Answering queries using views: A survey. The VLDB Journal, 10(4): 270-294, December 2001. ISSN 1066-8888.

Tom Heath and Christian Bizer. Linked Data: Evolving the Web into a Global Data Space. Morgan \& Claypool, 1st edition, 2011a. ISBN 9781608454303. URL http://linkeddatabook.com/.

Tom Heath and Christian Bizer. Linked data: Evolving the web into a global data space. Synthesis lectures on the semantic web: theory and technology, 1(1):1-136, 2011b.

Jan Hegewald, Felix Naumann, and Melanie Weis. Xstruct: efficient schema extraction from multiple and large xml documents. In Data Engineering Workshops, 2006. Proceedings. 22nd International Conference on, pages 81-81. IEEE, 2006.

Knut Hegna and Eeva Murtomaa. Data mining marc to find: Frbr? International Cataloguing and Bibliographic Control, 32(3):52-55, 2003.

Thomas B Hickey, Edward T O'Neill, and Jenny Toves. Experiments with the IFLA functional requirements for bibliographic records (FRBR). D-Lib magazine, 8(9): $1-13,2002$.

Matthew Hoffman, Francis R Bach, and David M Blei. Online learning for latent dirichlet allocation. In advances in neural information processing systems, pages 856864, 2010.

Liangjie Hong and Brian D Davison. Empirical study of topic modeling in twitter. In Proceedings of the First Workshop on Social Media Analytics, pages 80-88. ACM, 2010.

Maia Hristozova and Leon Sterling. An extreme method for developing lightweight ontologies. In Workshop on Ontologies in Agent Systems, 1st International Joint Conference on Autonomous Agents and Multi-Agent Systems, 2002. 
Noor Huijboom and Tijs Van den Broek. Open data: an international comparison of strategies. European journal of ePractice, 12(1):1-13, 2011.

Bernadette Hyland, Ghislain Atemezing, and Boris Villazón-Terrazas. Best practices for publishing linked data. W3C Working Group Note, 2014.

IFLA. Functional Requirements for Bibliographic Records: Final Report. IFLA Study Group on the Functional Requirements for Bibliographic Records, UBCIM publications ; new series, vol. 19 (Amended and corrected) edition, 2009. URL http: //www. ifla.org/files/cataloguing/frbr/frbr_2008.pdf.

IFLA. ISBD: International Standard Bibliographic Description: Consolidated Edition. Berlin Boston, Mass. De Gruyter Saur. IFLA series on bibliographic control, v. 34., 2011.

Peter Ingwersen and Kalervo Jarvelin. The Turn: Integration of Information Seeking and Retrieval in Context. Springer, first edition, 2005.

Robert Isele, Anja Jentzsch, Chris Bizer, and Julius Volz. Silk - A Link Discovery Framework for the Web of Data, January 2011.

ISO 2709. Information and documentation - Format for information exchange, 1996.

Krzysztof Janowicz, Pascal Hitzler, Benjamin Adams, Dave Kolas, Charles Vardeman, et al. Five stars of linked data vocabulary use. Semantic Web, 5(3):173-176, 2014.

Michael I Jordan, Zoubin Ghahramani, Tommi S Jaakkola, and Lawrence K Saul. An introduction to variational methods for graphical models. Machine learning, 37(2): 183-233, 1999.

Maulik R Kamdar, Tania Tudorache, and Mark A Musen. Investigating term reuse and overlap in biomedical ontologies. In Proceedings of the 6th International Conference on Biomedical Ontology, ICBO 2015, Lisbon, Portugal, July 27-30, 2015., 2015.

Diane Kelly. Methods for evaluating interactive information retrieval systems with users. Foundations and Trends in Information Retrieval, 3(1-2):1-224, 2009.

Henry F Korth and Mark A Roth. Query languages for nested relational databases. Springer, 1989.

Joseph B Kruskal. Multidimensional scaling by optimizing goodness of fit to a nonmetric hypothesis. Psychometrika, 29(1):1-27, 1964. 
Jey Han Lau, David Newman, and Timothy Baldwin. Machine reading tea leaves: Automatically evaluating topic coherence and topic model quality. In Proceedings of the Association for Computational Linguistics, pages 530-539, 2014.

Jianhua Lin. Divergence measures based on the shannon entropy. Information Theory, IEEE Transactions on, 37(1):145-151, 1991.

Fadi Maali, John Erickson, and Phil Archer. Data catalog vocabulary (dcat). W3C Recommendation, 2014.

Alexander Maedche and Steffen Staab. Measuring similarity between ontologies. In Knowledge engineering and knowledge management: Ontologies and the semantic web, pages 251-263. Springer, 2002.

Akifumi Makinouchi. A consideration on normal form of not-necessarily-normalized relation in the relational data model. In $V L D B$, volume 77, pages 447-453. Citeseer, 1977.

Hugo Miguel Álvaro Manguinhas, Nuno Miguel Antunes Freire, and José Luis Brinquete Borbinha. Frbrization of marc records in multiple catalogs. In Proceedings of the 10th annual joint conference on Digital libraries, pages 225-234. ACM, 2010.

Sergey Melnik, Andrey Gubarev, Jing Jing Long, Geoffrey Romer, Shiva Shivakumar, Matt Tolton, and Theo Vassilakis. Dremel: interactive analysis of web-scale datasets. Proceedings of the VLDB Endowment, 3(1-2):330-339, 2010.

Franck Michel, Loïc Djimenou, Catherine Faron-Zucker, and Johan Montagnat. xr2rml: Non-relational databases to rdf mapping language. Technical Report ISRNI3S/RR 2014-04-FR, CNRS., 2015.

David Mimno, Hanna M Wallach, Edmund Talley, Miriam Leenders, and Andrew McCallum. Optimizing semantic coherence in topic models. In Proceedings of the Conference on Empirical Methods in Natural Language Processing, pages 262-272. Association for Computational Linguistics, 2011.

Elena Montiel-Ponsoda, Daniel Vila-Suero, Boris Villazón-Terrazas, Gordon Dunsire, Elena Escolano Rodríguez, and Asunción Gómez-Pérez. Style guidelines for naming and labeling ontologies in the multilingual web. In Proceedings of the 2011 International Conference on Dublin Core and Metadata Applications. Dublin Core Metadata Initiative, 2011. 
Andrea Moro, Alessandro Raganato, and Roberto Navigli. Entity Linking meets Word Sense Disambiguation: a Unified Approach. Transactions of the Association for Computational Linguistics (TACL), 2:231-244, 2014.

Roberto Navigli. Word sense disambiguation: A survey. ACM Computing Surveys (CSUR), 41(2):10, 2009.

Roberto Navigli and Simone Paolo Ponzetto. BabelNet: The automatic construction, evaluation and application of a wide-coverage multilingual semantic network. Artificial Intelligence, 193:217-250, 2012.

Axel-Cyrille Ngonga Ngomo and Sören Auer. Limes - a time-efficient approach for large-scale link discovery on the web of data. In Proceedings of IJCAI, 2011.

Kamal Nigam, Andrew Kachites McCallum, Sebastian Thrun, and Tom Mitchell. Text classification from labeled and unlabeled documents using em. Machine learning, 39(2-3):103-134, 2000.

Natalya F Noy and Deborah L McGuinness. Ontology development 101: A guide to creating your first ontology, 2001.

IFLA Study Group on the Functional Requirements for Bibliographic Records. Functional requirements for bibliographic records : final report. 2009. URL http: //www. ifla.org/files/cataloguing/frbr/frbr_2008.pdf.

Larry Page, S Brin, R Motwani, and T Winograd. Pagerank: Bringing order to the web. Technical report, Stanford Digital Libraries Working Paper, 1997.

Valéria M Pequeno, Vania MP Vidal, Marco A Casanova, Luís Eufrasio T Neto, and Helena Galhardas. Specifying complex correspondences between relational schemas and $\mathrm{rdf}$ models for generating customized $\mathrm{r} 2 \mathrm{rml}$ mappings. In Proceedings of the 18th International Database Engineering \& Applications Symposium, pages 96-104. ACM, 2014.

Gary Perlman. Practical usability evaluation. In CHI '97: CHI '97 extended abstracts on Human factors in computing systems, pages 168-169, New York, NY, USA, 1997. ACM. ISBN 0-89791-926-2. doi: http://doi.acm.org/10.1145/1120212.1120326. Los Angeles, USA. April 18-23. 
H. Sofia Pinto, Steffen Staab, and Christoph Tempich. DILIGENT: Towards a finegrained methodology for DIstributed, Loosely-controlled and evolvInG Engineering of oNTologies. In Proceedings of the 16th Eureopean Conference on Artificial Intelligence, ECAI'2004, including Prestigious Applicants of Intelligent Systems, PAIS 2004, Valencia, Spain, August 22-27, 2004, pages 393-397, 2004.

Antonella Poggi, Domenico Lembo, Diego Calvanese, Giuseppe De Giacomo, Maurizio Lenzerini, and Riccardo Rosati. Linking data to ontologies. In Journal on data semantics $X$, pages 133-173. Springer, 2008.

Robert Porzel and Rainer Malaka. A task-based approach for ontology evaluation. In ECAI Workshop on Ontology Learning and Population, Valencia, Spain. Citeseer, 2004.

María Poveda-Villalón, Bernard Vatant, Mari Carmen Suárez-Figueroa, and Asunción Gómez-Pérez. Detecting good practices and pitfalls when publishing vocabularies on the web. 2013.

María Poveda-Villalón, Asunción Gómez-Pérez, and Mari Carmen Suárez-Figueroa. Oops!(ontology pitfall scanner!): An on-line tool for ontology evaluation. International Journal on Semantic Web and Information Systems (IJSWIS), 10(2):7-34, 2014.

Valentina Presutti, Eva Blomqvist, Enrico Daga, and Aldo Gangemi. Pattern-Based Ontology Design. In María del Carmen Suárez-Figueroa, Asunción Gómez-Pérez, Enrico Motta, and Aldo Gangemi, editors, Ontology Engineering in a Networked World., pages 35-64. Springer, 2012.

Filip Radulovic, María Poveda-Villalón, Daniel Vila-Suero, Víctor Rodríguez-Doncel, Raúl García-Castro, and Asunción Gómez-Pérez. Guidelines for linked data generation and publication: An example in building energy consumption. Automation in Construction, 57:178 - 187, 2015. ISSN 0926-5805.

Shiyali Ramamrita Ranganathan. The five laws of library science. Madras Library Association, Madras, 1931.

Víctor Rodríguez Doncel, Asunción Gómez-Pérez, and Serena Villata. A dataset of rdf licenses. 2014.

Mark A Roth, Henry F Korth, and Don S Batory. Sql/nf: a query language for $\neg 1 \mathrm{nf}$ relational databases. Information systems, 12(1):99-114, 1987. 
Satya S Sahoo, Wolfgang Halb, Sebastian Hellmann, Kingsley Idehen, Ted Thibodeau Jr, Sören Auer, Juan Sequeda, and Ahmed Ezzat. A survey of current approaches for mapping of relational databases to rdf. W3C RDB2RDF Incubator Group Report, pages 113-130, 2009.

Gerard Salton and J Michael. Mcgill. Introduction to modern information retrieval, pages 24-51, 1983.

Ricardo Santos, Ana Manchado, and Daniel Vila-Suero. Datos.bne.es: a lod service and a frbr-modelled access into the library collections. In IFLA World Library International Conference. Cape Town, South Africa, 2015.

Advait Sarkar. Spreadsheet interfaces for usable machine learning. In Visual Languages and Human-Centric Computing (VL/HCC), 2015 IEEE Symposium on, pages 283-284. IEEE, 2015.

Johann Schaible, Thomas Gottron, and Ansgar Scherp. Survey on common strategies of vocabulary reuse in linked open data modeling. In The Semantic Web: Trends and Challenges, pages 457-472. Springer, 2014.

Craig W Schnepf. Sql/nf translator for the triton nested relational database system. Technical report, DTIC Document, 1990.

Kunal Sengupta, Peter Haase, Michael Schmidt, and Pascal Hitzler. Editing r2rml mappings made easy. In International Semantic Web Conference (Posters, Demos), pages 101-104, 2013.

Juan F Sequeda, Marcelo Arenas, and Daniel P Miranker. On directly mapping relational databases to $\mathrm{rdf}$ and owl. In Proceedings of the 21st international conference on World Wide Web, pages 649-658. ACM, 2012.

Juan F Sequeda, Marcelo Arenas, and Daniel P Miranker. Obda: Query rewriting or materialization? in practice, both! In The Semantic Web-ISWC 2014, pages 535-551. Springer, 2014.

James Shore and Shane Warden. The art of agile development. O'Reilly, 2007. ISBN 978-0-596-52767-9. URL http://www. oreilly.de/catalog/9780596527679/ index.html. 
Pavel Shvaiko and Jérôme Euzenat. Ontology matching: state of the art and future challenges. Knowledge and Data Engineering, IEEE Transactions on, 25(1):158-176, 2013.

Agnès Simon, Romain Wenz, Vincent Michel, and Adrien Di Mascio. Publishing bibliographic records on the web of data: opportunities for the bnf (french national library). In The Semantic Web: Semantics and Big Data, pages 563-577. Springer, 2013.

Jason Slepicka, Chengye Yin, Pedro Szekely, and Craig A Knoblock. Kr2rml: An alternative interpretation of $\mathrm{r} 2 \mathrm{rml}$ for heterogeneous sources. In Proceedings of the 6th International Workshop on Consuming Linked Data, COLD 2015, Bethlehem, Pennsylvania, USA, October 2015. CEUR Workshop Proceedings 1426, CEUR Workshop Proceedings 1426.

Steffen Staab, Rudi Studer, Hans-Peter Schnurr, and York Sure. Knowledge processes and ontologies. IEEE Intelligent systems, 16(1):26-34, 2001.

Giorgos Stoilos, Giorgos Stamou, and Stefanos Kollias. A string metric for ontology alignment. In The Semantic Web-ISWC 2005, pages 624-637. Springer, 2005.

Louise T Su. Evaluation measures for interactive information retrieval. Information Processing \& Management, 28(4):503-516, 1992.

Mari Carmen Suárez-Figueroa, Eva Blomqvist, Mathieu d'Aquin, Mauricio Espinoza, Asunción Gómez-Pérez, Holger Lewen, Igor Mozetic, Raúl Palma, María Poveda, Margherita Sini, Boris Villazón-Terrazas, Fouad Zablith, and Martin Dzbor. D5.4.2 Revision and Extension of the NeOn Methodology for Building Contextualized Ontology Networks. Technical report, Universidad Politécnica de Madrid (UPM), 2009. NeOn Project. http://www. neon-project. org.

Mari Carmen Suárez-Figueroa, Asunción Gómez-Pérez, and Boris Villazón-Terrazas. How to write and use the ontology requirements specification document. In Proceedings of the Confederated International Conferences, CoopIS, DOA, IS, and ODBASE 2009 on On the Move to Meaningful Internet Systems: Part II, OTM '09, pages 966-982, Berlin, Heidelberg, 2009. Springer-Verlag. ISBN 978-3-642-05150-0.

Mari Carmen Suárez-Figueroa, Asunción Gómez-Pérez, and Mariano FernándezLópez. The neon methodology framework: A scenario-based methodology for ontology development. Applied Ontology, (Preprint):1-39, 2015. 
Naimdjon Takhirov. Extracting knowledge for cultural heritage knowledge base population. Doctoral dissertation, 2013.

Naimdjon Takhirov, Trond Aalberg, Fabien Duchateau, and Maja Zumer. Frbr-ml: A frbr-based framework for semantic interoperability. Semantic Web, 3(1):23-43, 2012.

Samir Tartir, I Budak Arpinar, Michael Moore, Amit P Sheth, and Boanerges AlemanMeza. Ontoqa: Metric-based ontology quality analysis. 2005.

Stan J. Thomas and Patrick C. Fischer. Nested relational structures. Advances in Computing Research, 3:269-307, 1986.

Jeffrey D. Ullman. Information integration using logical views. Theoretical Computer Science, 239(2):189 - 210, 2000. ISSN 0304-3975.

Pierre-Yves Vandenbussche and Bernard Vatant. Linked open vocabularies. ERCIM news, 96:21-22, 2014.

Daniel Vila-Suero and Elena Escolano. Linked Data at the Spanish National Library and the Application of IFLA RDFS Models. In IFLA SCATNews Number 35, 2011. URL http://www.ifla.org/files/cataloguing/scatn/ scat-news-35.pdf.

Daniel Vila Suero and Elena Escolano Rodríguez. Linked Data at the Spanish National Library and the application of IFLA RDFS models. IFLA ScatNews, (35):5-6, 2011.

Daniel Vila-Suero and Asunción Gómez-Pérez. datos. bne. es and marimba: an insight into library linked data. Library Hi Tech, 31(4):575-601, 2013.

Daniel Vila-Suero, Boris Villazón-Terrazas, and Asunción Gómez-Pérez. datos. bne. es: A library linked dataset. Semantic Web, 4(3):307-313, 2013.

Daniel Vila-Suero, Asunción Gómez-Pérez, Elena Montiel-Ponsoda, Jorge Gracia, and Guadalupe Aguado-de Cea. Publishing linked data on the web: The multilingual dimension. In Paul Buitelaar and Philipp Cimiano, editors, Towards the Multilingual Semantic Web, pages 101-117. Springer Berlin Heidelberg, 2014a. ISBN 978-3-662-43584-7.

Daniel Vila-Suero, Víctor Rodríguez-Doncel, Asuncion Gómez-Pérez, Philipp Cimiano, John McCrae, and Guadalupe Aguado-de Cea. 3ld: Towards high quality, industry-ready linguistic linked linguistic data. European Data Forum 2014, 2014b. 
Boris Villazón-Terrazas, Luis Vilches, Oscar Corcho, and Asunción Gómez-Pérez. Methodological Guidelines for Publishing Government Linked Data. Springer, 2011.

Boris Villazón-Terrazas, Daniel Vila-Suero, Daniel Garijo, Luis M Vilches-Blázquez, Maria Poveda-Villalón, José Mora, Oscar Corcho, and Asunción Gómez-Pérez. Publishing linked data-there is no one-size-fits-all formula. In European Data Forum, 2012.

R Hevner von Alan, Salvatore T March, Jinsoo Park, and Sudha Ram. Design science in information systems research. MIS quarterly, 28(1):75-105, 2004.

W3C. Describing linked datasets with the void vocabulary. URL http://www.w3. org/TR/void/.

W3C. Library linked data incubator group: Final report. Technical report, World Wide Web Consortium (W3C), 2011.

Yimin Wang, Johanna Völker, and Peter Haase. Towards semi-automatic ontology building supported by large-scale knowledge acquisition. In AAAI Fall Symposium On Semantic Web for Collaborative Knowledge Acquisition, volume 6, page 06, 2006.

Jianshu Weng, Ee-Peng Lim, Jing Jiang, and Qi He. Twitterrank: finding topicsensitive influential twitterers. In Proceedings of the third ACM international conference on Web search and data mining, pages 261-270. ACM, 2010.

Ya Xu and David Mease. Evaluating web search using task completion time. In Proceedings of the 32nd international ACM SIGIR conference on Research and development in information retrieval, pages 676-677. ACM, 2009.

Wayne Xin Zhao, Jing Jiang, Jianshu Weng, Jing He, Ee-Peng Lim, Hongfei Yan, and Xiaoming Li. Comparing twitter and traditional media using topic models. In Advances in Information Retrieval, pages 338-349. Springer, 2011. 